\title{
GLOBAL PROTEOME RESPONSE OF HUMAN CANCER CELL LINES TO LOW DOSE elF4E/eIF4G INHIBITION
}

Rory Nicholas Besaans

Supervised by Dr Paul Teesdale-Spittle

A thesis submitted to the Victoria University of Wellington in fulfilment of the degree of Master of Science in Biotechnology

Victoria University of Wellington 





\subsection{Abstract}

Cachexia is a debilitating muscle wasting disease and co-morbidity strongly associated with chronic inflammatory conditions such as cancer, chronic heart failure, chronic obstructive pulmonary disease and sepsis. Cachexia has a strong negative impact on quality of life and research suggests that $20 \%$ of cancer patients will die of cachexia. Translation initiation is the most highly regulated step of protein synthesis and the eukaryotic initiation factor $4 \mathrm{~F}$ (elF4F) translation initiation complex is the gatekeeper of this process; the elF4F complex is composed of elFG, a scaffolding protein, elF4E, an mRNA cap-recognition protein and elF4A, an RNA helicase. Inhibition of elF4A by pateamine $A$ has been shown to rescue muscle wasting in vitro and in vivo, this result has been reproduced with other elF4A inhibitors. Pateamine $A$ is a sponge-derived natural product with nanomolar toxicity to cancer cells. Surprisingly, at doses well below its anti-neoplastic activity it exerts distinct effects on cachexia. The research in this thesis follows on from previous work in our laboratory with pateamine $A$ in human cell lines. Work on the effects of pateamine $A$ on the proteome suggests that not all the proteins changing in expression are explainable by stressing the translation initiation complex. A model by which motifs in the $5^{\prime}$ UTRs of transcripts are a recognised and removed from the system in a selective manner could help explain these effects. We aimed to target elF4E, another component of the elF4F system, with two compounds to see if a comparable dose of elF4E inhibitors could elicit a pateamine-like response. DMSO, a solvent used extensively in this thesis, had unexpected effects on translation. We conclude that 4E1RCat, a compound developed as a selective inhibitor of elF4E, is not likely to be useable in further work, due to its window of activity coinciding with an unacceptable concentration of DMSO. Ribavirin, our second compound, showed a proteomic response consistent with its classification as an elF4E translation initiation inhibitor. The proteome response seen with our elF4E inhibitors is consistent with disruption of translation initiation. However, the data for 4E1RCat was deemed untrustworthy in the wake of revelations that DMSO, the vehicle in which it is dissolved, exerts an almost identical response. From the results obtained, it was not possible to confidently test whether protein downregulation occurred in response to a 5'UTR sequence motif, as seen for inhibitors of elF4A. Coupled with the uncertainty associated with the 4E1Rcat results, there were relatively few downregulated proteins from the treatments, and many of these could be explained by the direct biological response to the function of the compound in the treatment. All in all, we have obtained new insights into the effects of DMSO on the proteome which will aid further experimentation. This thesis has laid the groundwork for further investigation of the effects of elF $4 \mathrm{~F}$ inhibition in the context of better understanding the remediation of cachexia through the elF4F system. 


\subsection{Acknowledgements}

I cannot remember a time when I have felt so utterly burnt-out, while simulatenously feeling such a great sense of contentment. In the words of the late Christopher Hitchens "I burned the candle at both ends ... and it gave a lovely light". This has truly been an amazing and formative experience for me and there are simply too many people to thank. That being said there are some people whose contribution to both my development as an individual, and to this thesis warrants extra thanking.

Paul, I feel like you are too modest to want or expect credit for someone else's achievement, but it would be wrong for me to not attribute part of my success to your guidance, mentorship and seemingly infinite patience. I cannot express how grateful I am for all the times you walked me through concepts I should know and explained them to me in words I could understand. I try my best to extend this same patience to those who require my help and guidance. Needless to say, I have a long way to go, but it has been awesome to have such a great role model to show how to be an excellent teacher and mentor. I think some of why the School of Biological Sciences is such a wellfunctioning department and such a friendly and collaborative place is attributable to your outlook and the outlook of others like you, such as Anne La Flamme, John Miller and Peter Pfeffer all of whom I remember fondly from my days as an undergraduate. I haven't forgotten about you Melanie, Andrew and Darren, thank you for being great teachers and academics too.

Vimal, many of the things I have thanked Paul for are things you provided me with too, in a different sense perhaps. Thank you for all the help, thoughtful conversations and laughs. It's been a pleasure sharing an office with you. Clint, I get the feeling that I was in some way a kindred proteomics-spirit, if you ever get a chance, look for the CLINT1 node I left in the STRING diagram in homage. It should be in the DMSO downregulated hits diagram! The one node in SBS with no friends! I hope you remember the joke for my sake! Richard, thank you for always helping me out with your process, you saved me innumerable headaches. Leti, thanks for being so damn efficient and organised and helping me out in tissue culture. Carl, thanks for encouraging me, perhaps unintentionally, not to pander or clamour for attention and for reinforcing my belief that being nice doesn't make you a good person, you are not nice, and undeniably a good person. I miss your sense of humour greatly.

Ali, although I may not express it, I am incredibly sad that we are no longer on speaking terms. I am so glad that we got to know each other so well this year, you were definitely an overall net positive addition to my life. I cherish the memories we share. Alvey, thanks for being such good fun, and always being down to nerd-out about science and the like. Sonja, despite being so different in our approaches to life, I love your take on things, despite it being blunt at times. Don't change too much, I care about you deeply and hope your new chapter in America works out well for you!

Reigh, Stephen, John, Holly, Sam, Alex and Madge, thank you for being such amazing friends, I am so glad that we are still friends this far out of our undergraduate degrees. I've never been a clique person, but l'd clique with you guys any day!

To my family, Mark, Ginelle, Kevin and Rob, thank you for putting up with me, I suspect I have not been the most fun to be around in the last year. I miss you all dearly and hope to be able to spend more time with you in the future. I am slowly realising how important my family is to me. Thank you for always being there for me.

Lots of love, Rory 


\subsection{Abbreviations}

\begin{tabular}{|c|c|}
\hline BMI & Body mass index \\
\hline $\mathrm{CHCA}$ & a-Cyano-4-hydroxycinnamic acid \\
\hline $\mathrm{CHX}$ & Cycloheximide \\
\hline CID & Collision-induced dissociation \\
\hline DMSO & Dimethyl sulfoxide \\
\hline $\mathrm{dH}_{2} \mathrm{O}$ & Distilled water \\
\hline elF4(A/E/F/G) & Eukaryotic initiation factor $4(\mathrm{~A} / \mathrm{E} / \mathrm{F} / \mathrm{G})$ \\
\hline FBS & Fetal bovine serum \\
\hline FDR & False discovery rate/s \\
\hline GO & Gene ontology \\
\hline GOAT & Gradient optimisation and analysis tool \\
\hline iBAQ & Intensity based absolute quantification \\
\hline LBM & Lean body mass \\
\hline LC & Liquid chromatography \\
\hline LTQ & Linear trap quadrupole \\
\hline MALDI-TOF & Matrix-assisted laser desorption/ionisation - time of flight \\
\hline MS & Mass spectrometer/spectrometry \\
\hline MS/MS & Tandem mass spectrometry \\
\hline MTT & 3-(4,5-dimethylthiazol-2-yl)-2,5-diphenyltetrazolium bromide \\
\hline PatA & Pateamine A \\
\hline PBS & Phosphate buffered saline \\
\hline PEG & Polyethylene glycol \\
\hline PSM & Peptide spectrum match \\
\hline RBV & Ribavirin \\
\hline RIPA buffer & Radioimmunoprecipitation assay buffer \\
\hline SDC & Sodium deoxycholate \\
\hline SDS & Sodium dodecyl sulfate \\
\hline STAT3 & Signal transducer and activator of transcription 3 \\
\hline TIC & Total ion current \\
\hline
\end{tabular}




\subsection{Table of contents}

\section{Contents}

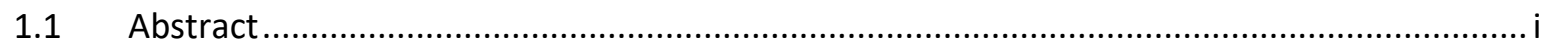

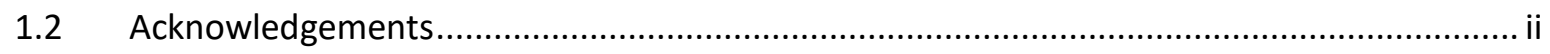

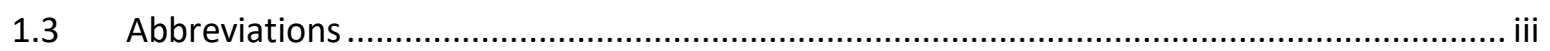

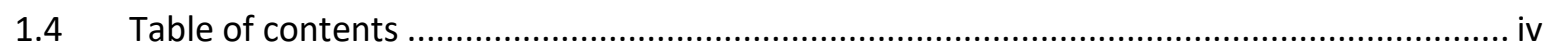

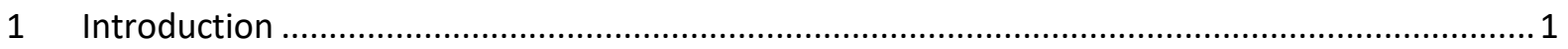

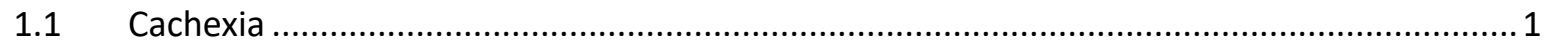

1.1.1 Effects on survival prospects and quality of life ….................................................... 1

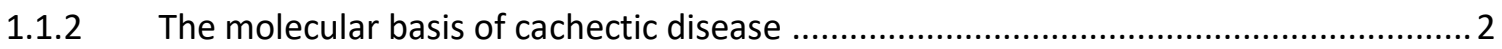

1.1.3 Cachexia management and current therapeutic approaches .....................................2

1.1.4 The lack of progress in the realm of cachexia treatment is a driver of our research ..... 3

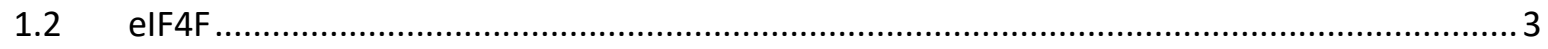

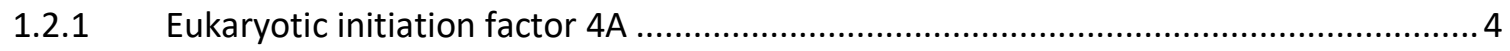

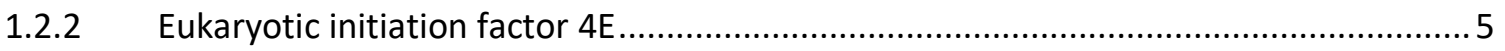

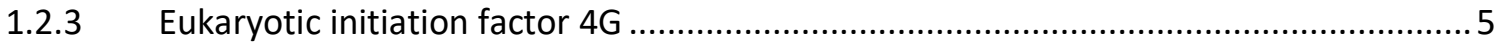

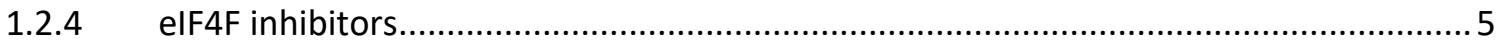

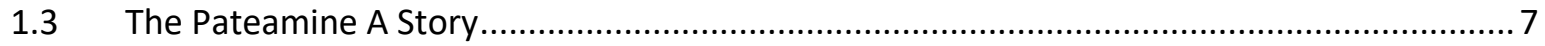

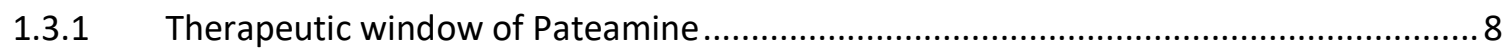

1.3.2 The problem: Pateamine availability …........................................................... 9

1.3.3 What can Pateamine and elF4A inhibition teach us about cachexia............................. 9

1.3.4 How does elF4A inhibition ameliorate cachexia at the molecular level? ...................... 10

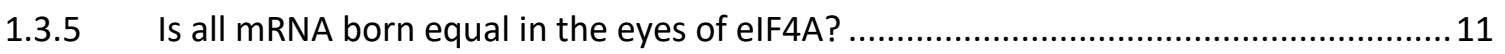

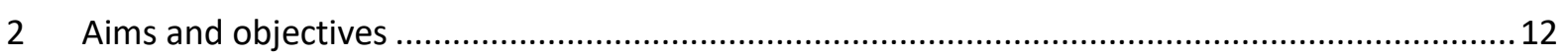

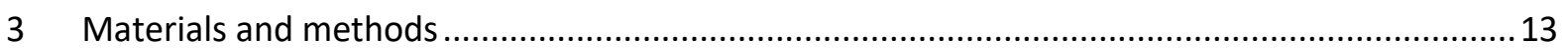

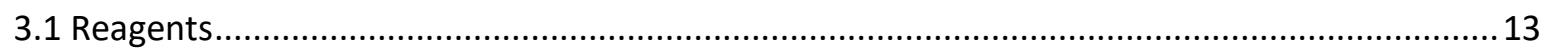

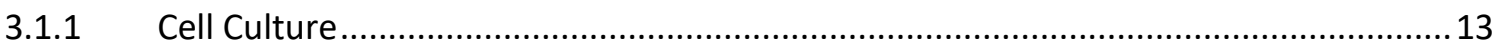

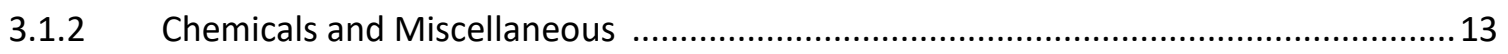

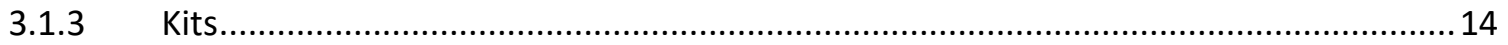

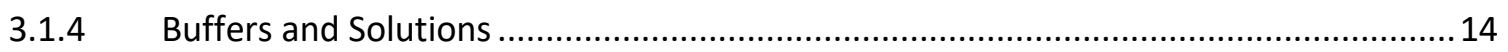

3.2 Drug stocks (CHX, RBV and 4E1RCAT) - preparation and storage ..................................... 15

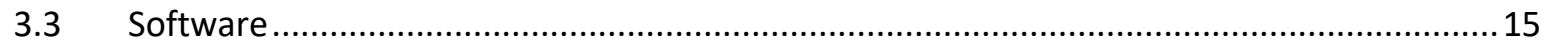

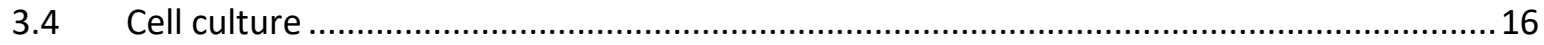

3.4.1 Cell culture - strains and maintenance conditions HL60 and HT29 ............................ 16 


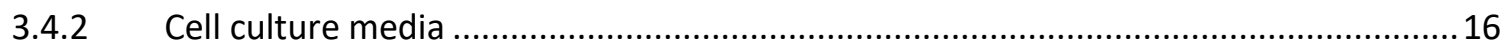

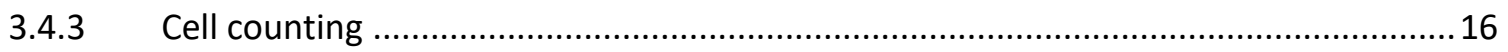

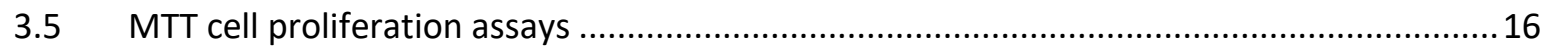

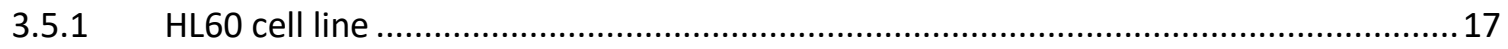

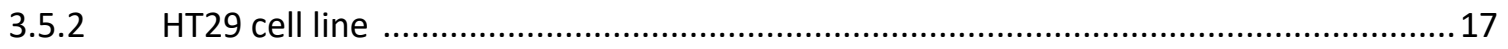

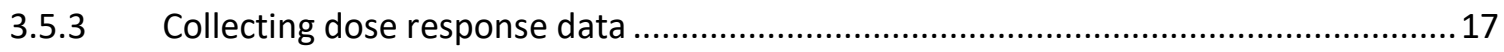

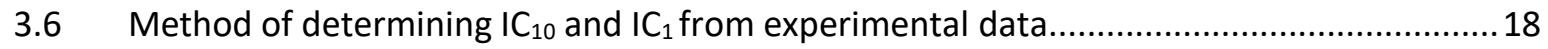

3.7 Preparing cells for pharmacological challenge ............................................................... 18

3.8 Sample preparation for use in LC-MS/MS ...................................................................... 18

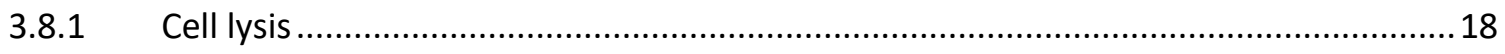

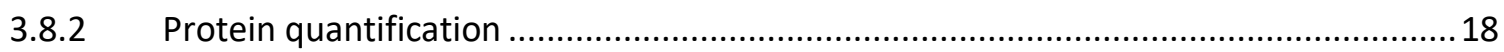

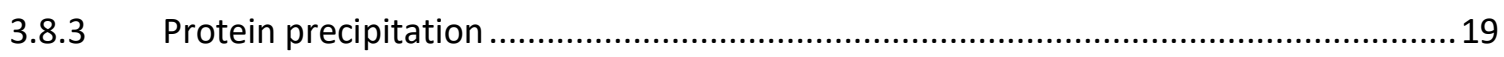

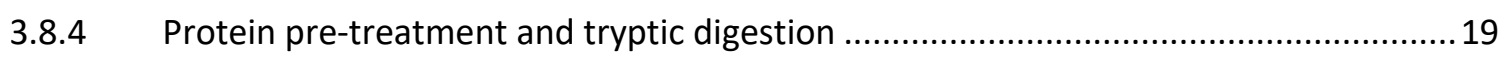

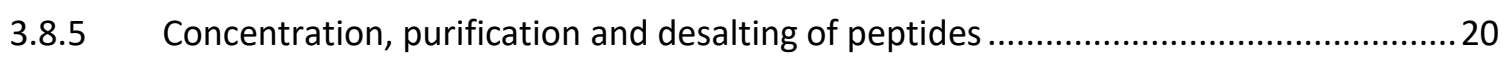

3.9 Matrix assisted laser desorption ionisation MS - time of flight (MALDI-TOF/MS)...............20

3.10 LC-MS/MS settings and data analysis parameters............................................................ 21

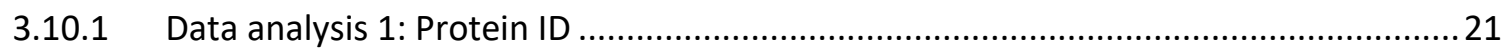

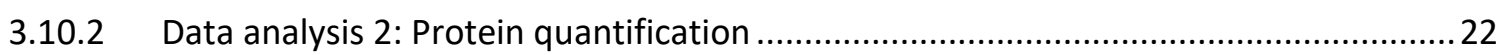

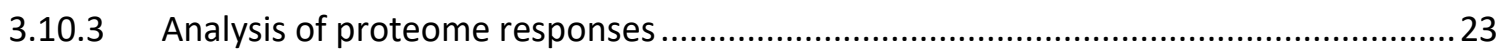

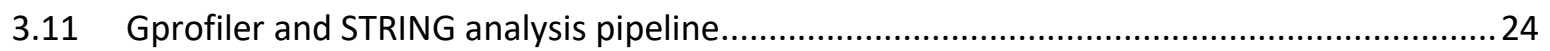

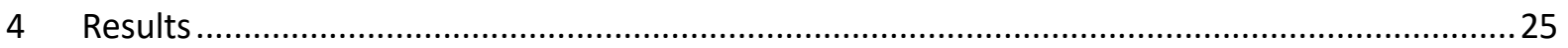

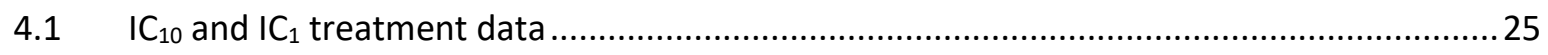

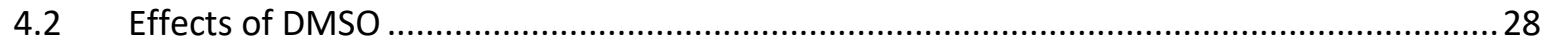

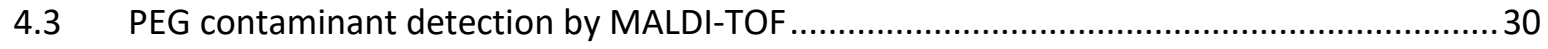

4.4 A side-by-side comparison of protein precipitation methods .............................................. 34

4.5 Assessing the effectiveness of lysis solutions without non-ionic detergents........................35

4.6 Mass spectrometry optimisation - chromatograms …....................................................... 36

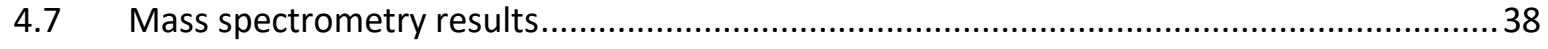

4.7.1 Second set of MS results: HT29 cells - Untreated, CHX (0.043 $\mu \mathrm{M})$ and RBV (11.1 $\mu \mathrm{M})$..

4.7.2 Third set of results: HT29 cells - DMSO control (1\%) and 4E1RCat $(40 \mu \mathrm{M}) \ldots \ldots \ldots \ldots . . . . . .39$

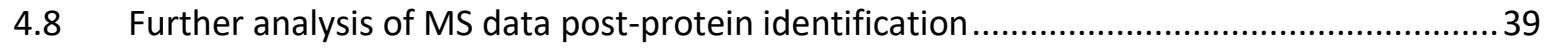

4.8.1 Gene ontological enrichment analysis using G profiler .............................................. 40

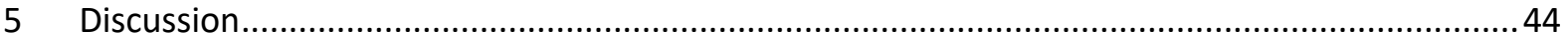

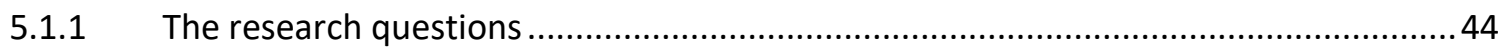

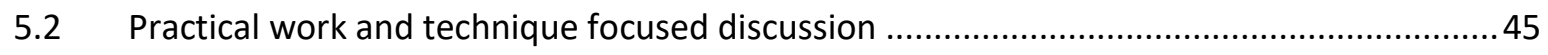




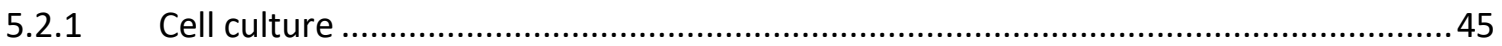

5.2.2 Cell lysis and protein handling optimisation ................................................................. 48

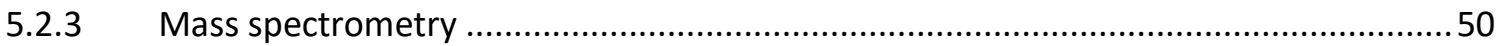

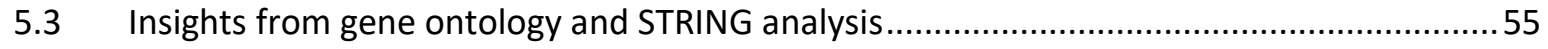

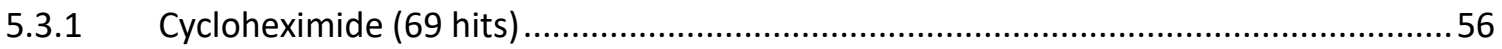

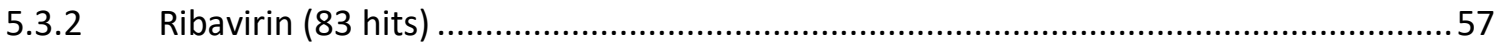

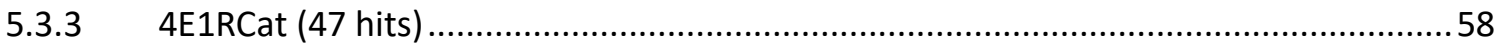

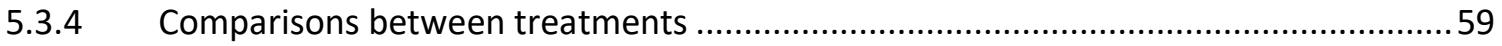

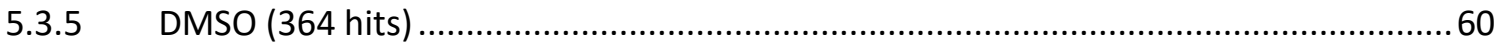

5.3.6 Summary of insights from GO and STRING analysis of treatments .............................62

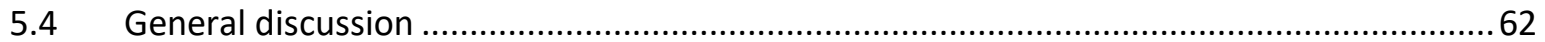

5.4.1 The purpose and power of a whole-proteome experiment ........................................62

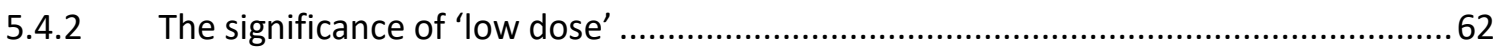

5.4.3 4E1RCat and separating the DMSO effects from drug treatment effects ....................62

5.4.4 Ribavirin, a 'dirty' drug with multiple interactions ..................................................... 63

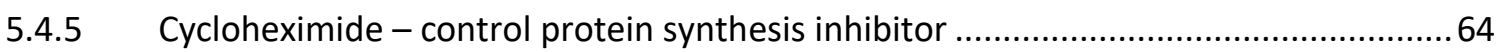

5.4.6 Regulatory network influence on translational output ................................................6 65

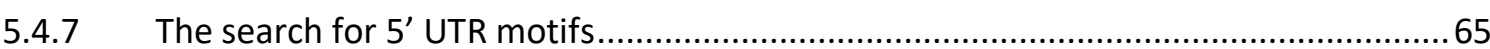

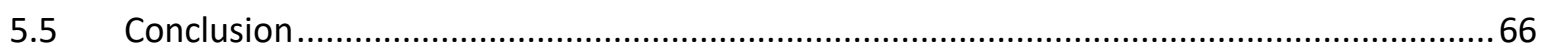

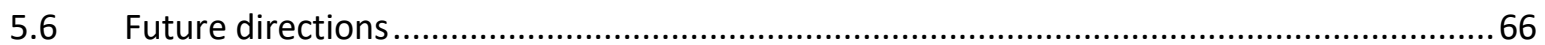

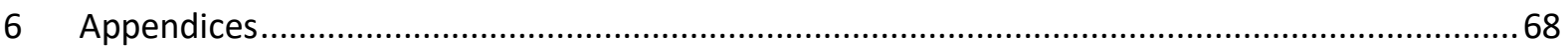

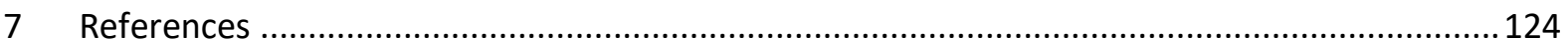




\section{Introduction}

Cachexia is a wasting disorder that affects millions of people world-wide and is generally associated with chronic inflammatory conditions. Research suggests it could be responsible for as many as 1 in 5 deaths in cancer patients. Systemic inflammation is central to the development of cachexia, and pro-inflammatory cytokines are a feature of all forms of cachexia. Translation initiation is controlled by the elF4F complex and is the most highly regulated step in protein expression and is the gateway to cellular production of protein. The three-part elF4F complex consists of a scaffolding protein, elF4G, a cap-recognition protein, elF4E, and the helicase elF4A, responsible for melting secondary structure and giving the ribosome access to mRNAs that might otherwise have their translation hindered. Inhibition of elF4A, by the marine natural product pateamine A (PatA) has recently been demonstrated to reverse cachexia in vitro, in lab-grown muscle fibres and in vivo in mice. Pateamine $A$ is an exceptionally potent anti-cancer compound isolated from marine sponges in New Zealand and has a unique method of disrupting translation. PatA has been shown to have applications outside the realm of cancer treatment, at doses well-below its anti-cancer effects. Pat $A$ is unfortunately in short supply, and despite being the first therapy to address the cachexia at the molecular level, the transition of PatA from pre-clinical to clinical trials has been delayed. Efforts are being made to find a commercially viable method of procuring PatA, however, in the meantime lessons can be learned from the molecular effects of PatA on the elF4F system. The precise mechanism by which PatA rescues cachexia has not been elucidated and it is not known whether inhibiting elF4A is solely responsible, or if the elF4F complex is also involved. Our lab group has previously observed the effects of PatA on the proteome of a selection of human cell lines, the work in this thesis is done in parallel and mirrors the treatment conditions and cell lines used. This research aims, in part, to establish whether elF4F has a role in the anti-cachectic effects of elF4A inhibition. By partially disabling cap-recognition by inhibiting elF4E in human cell lines and observing the effects on the proteome, we hope to see whether there are shared outcomes between the cells subjected to inhibition of elF4E and elF4A.

\subsection{Cachexia}

Cachexia is a metabolic wasting disorder that results in the involuntary progressive loss of weight through atrophy of muscle and fat tissues. It is co-morbid with many chronic diseases, and its etiology is grounded in inflammatory processes, anorexia, and muscle and fat tissue catabolism (Argilés, Busquets, Stemmler, \& López-Soriano, 2014). In the United States alone approximately 27 million people suffer from cachexia with around 5.5 million requiring treatment (Morley, Thomas, \& Wilson, 2006). There are two clinical consensus definitions of cachexia. Fearon (2011) focus on weight loss, BMI, and sarcopenia, whilst Evans (2008) definition is based on decreased muscle strength, fatigue, anorexia, low fat-free mass index and abnormal biochemistry (an in increase in inflammatory markers; anemia; and low serum albumin. The Evans definition has been shown to be especially effective (Vanhoutte et al., 2016). The Evans' cohort identified chronic heart failure, chronic obstructive pulmonary disease (COPD), chronic kidney disease, chronic infection, sepsis and cancer as a set of disease states associated strongly with cachectic wasting. Loss of muscle, loss of fat, and anemia are key identifiers of cachexia (Evans et al., 2008).

\subsubsection{Effects on survival prospects and quality of life}

The most significant contributor to cachectic disease is COPD with 16 million sufferers, followed by heart failure with 4.8 million and rheumatoid arthritis at 2.1 million (Morley et al., 2006). In contrast, cancer cachexia at 1.4 million, ranks below even nursing home sufferers of cachexia at 1.6 million. The literature tends to focus heavily on cancer cachexia despite only about $6 \%$ of cachexia sufferers 
being in this group. Decreases in lean body mass (LBM) and functional impairment lead to a decreased quality of life. Along with this is increased risk of mortality, as shown in a study from 1932, documenting the cause of death in 500 cases of cancer with cachexia. In the study, fully one fifth of the cancer deaths were attributed to cachexia (Warren S, 1932).

\subsubsection{The molecular basis of cachectic disease}

Despite the complex and convoluted etiology of cachexia, research has implicated several molecular mechanisms and pathways of interest which are thought to account for the loss of lean muscle and fat tissues. Skeletal muscle accounts for $40 \%$ of an individuals' body mass and its loss is an obvious, visual sign of disease. However, focusing too heavily on skeletal muscle loss is misleading and does not address the nuances inherent in this disease state. A correct assessment of cachexia involves acknowledgement of the syndrome as multi-organ, multi-factorial and typified by abnormal biochemistry (Argilés et al., 2014). Cancers' meagre contribution to the overall number of cachexia sufferers' contrasts with the extensive insights the study of cancer cachexia has bestowed upon the field. 'Adipose browning' or conversion of white adipose tissue to brown adipose is driven by tumour secreted molecules including IL-6 from the inflammatory response, and parathyroid-hormonerelated protein (PTHrP) generally secreted by tumour cells. Neutralising PTHrP caused a decrease in both white adipose tissue browning and the loss of muscle mass in mice (Serkan Kir et al., 2014). UCP1, an uncoupling protein commonly known as thermogenin, causes increased levels of thermogenesis and is responsible for fat-mass loss and contributes to the wasting process. The expression of thermogenin in brown adipose is caused by conversion of white adipose to brown adipose by IL-6 and PTHrP. Weight loss is a common symptom of cachexia, as a function of a negative tilt on an individual's energy balance and homeostasis of muscle tissue caused by increased thermogenesis and increased levels of inflammatory cytokines. Commonly associated with cachexia is a loss of appetite, also indicating effects on the brain and hypothalamus and providing more support for the theory of cachexia as a multi-organ syndrome (Argilés et al., 2014). Decreased food intake is common in cancer patients and speeds up the decline of body mass. In these patients, weight loss is also driven by the high protein, glucose, and fat demands of tumours. Activated immune cells and tumours are the main producers of inflammatory cytokines in cancer cachexia. One of these cytokines, TNF- $\alpha$, is known to have direct catabolic effects on skeletal muscle and adipose tissue (Reid \& Li, 2001).

\subsubsection{Cachexia management and current therapeutic approaches}

The systemic inflammatory response seems to be the connection between the major pathologies that cause cachexia, and as such anti-inflammatory drugs are a logical way to counteract cachexia by reducing systemic inflammation. The most common way to achieve this is by suppression of proinflammatory cytokine production. TNF- $\alpha$ is a pro-inflammatory and pro-catabolic cytokine, in 2005 a randomised placebo controlled trial showed that thalidomide was well-tolerated and effective at attenuating weight loss, and LBM loss in pancreatic cancer patients suffering from cachexia (Gordon et al., 2005). Another suggested therapeutic option for addressing weight loss in cancer cachexia on a molecular level is $\beta$-adrenergic blockade. In a recent trial in severe chronic heart failure patients, carvedilol attenuated the development of and partially reversed cachexia (Clark et al., 2017). However, most research into cachexia treatments is aimed at management through symptomfocused therapies. Appetite stimulant therapies are a direct way of addressing loss of appetite often seen in cancer patients, as well as the weight loss associated with reduced food intake. These stimulants are therapeutic interventions that do not directly address cachexia on the molecular level but address the loss of fat and muscle tissue indirectly by addressing the weight loss associated with cachectic wasting. Megestrol acetate, an orally accessible synthetic progesterone, and L-carnitine have, in combination and individually, shown promise at helping with the loss of weight and LBM 
(Von Haehling \& Anker, 2015). Megestrol acetate has also shown promise in dealing with cachectic weight loss in children suffering from cancer and the weight loss associated with chemotherapy; there was a mean increase in LBM of $19.7 \%$ in comparison to the placebo group with a loss of $1.2 \%$ over the course of the 90 day trial period (Frey \& Davis, 2016). In terms of sarcopenia, nutritional support and exercise have marked effects on muscle loss; however, the effects of these measures are less clear in cachexia. A Cochrane meta-analysis of nutritional support and mortality in elderly people at risk of malnutrition indicates small benefits such as increased weight gain and a potential to lower the risk of complications in hospital conditions. The authors state that nutritional support may lower the risk of mortality in people who are malnourished (Milne, Potter, \& Avenell, 2005). Since cachexia is in many ways a catabolic syndrome, the use of anabolic steroids may seem like a logical way to counteract the disease. Unfortunately, anabolic steroid therapies are limited to 2week treatment periods due to concern about side effects (Von Haehling \& Anker, 2015; Yu et al., 2014) . In response, clinical trials of enobosarm, a selective androgen receptor modulator, in 2013 were completed and suggested some benefits (Dobs, 2013). However, no new clinical data has recently been reported.

\subsubsection{The lack of progress in the realm of cachexia treatment is a driver of our research}

The lack of a cachexia therapeutics is not a symptom of the biomedical science field's failure to explore therapeutic options. The effective treatment of cachexia is littered with the bodies of clinical trials showing modest to marginal improvements in cachexia endpoints. These endpoints include hand grip strength, performance in the 6-minute walk test, LBM and quality of life. Anamorelin, an appetite stimulant and anabolic therapeutic, showed marginal improvements in LBM, with no improvement in hand-grip strength or quality of life (Katakami et al., 2018; Von Haehling \& Anker, 2015). On 14 September 2017, the European Medicines Agency confirmed the refusal of marketing authorisation for amorelin in cachexia treatment. Frustratingly, reading between the lines is necessary with therapeutics in clinical trials, it takes a significant amount of time to work out if some of the promising clinical agents have progressed in the cachexia therapy space. This may be economically motivated as there is no incentive for a company to actively broadcast that a therapeutic in development has marginal effects on the disease it has been formulated to treat. Enobosarm seems to have stalled in clinical trials and may be awaiting regulatory approval to proceed. Taken together, the attempts at addressing cachexia have highlighted several therapeutic options for clinicians with modest improvements in symptomatic consequences of cachectic muscle wasting. Nonetheless, therapy addressing the underlying molecular cause of cachexia is still needed. Studies in murine models of cachexia and in vitro at the level of protein production using inhibitors of the translation initiation factor elF4A are a promising avenue to explore. The next step is to better understand the mechanistic underpinning of these effects, which may ultimately lead to effective treatments for this disease.

\section{2 elF4F}

The eukaryotic initiation factor $4 \mathrm{~F}$ (elF4F) has been described as a nexus for cancer therapy (Pelletier, Graff, Ruggero, \& Sonenberg, 2015). The elF4F complex is necessary for recruitment of mRNA to the ribosome in cap-dependent translation. It is composed of three components: eukaryotic initiation factors $4 \mathrm{~A}, 4 \mathrm{G}$, and $4 \mathrm{E}$. elF4A is an RNA helicase; elF4E, an $\mathrm{m7G}$ cap recognition protein, and elF4G a large scaffolding protein (Figure 1-1). Unlike other translation regulatory pathways, elF4F, with the exception of translation mediated by internal ribosome entry sites (IRESes), is an unavoidable 'bottleneck' that cancers have a hard time circumventing. Enzymes which regulate elF4F-mediated translation by phosphorylation, such as Mnk1 and 2, encounter redundancy issues whereby cancer cells can subvert the regulatory nature of these pathways by finding another way to favourably regulate key proteins that promote oncogenesis or maintenance of the cancer 
cell. For example, the elF4F complex is typically indispensable for translation and if Mnk1/2 inhibitors are being used to prevent activating the elF4F complex by phosphorylation, the cancer cell will find another way to phosphorylate and activate elF4F to meet its protein requirements.

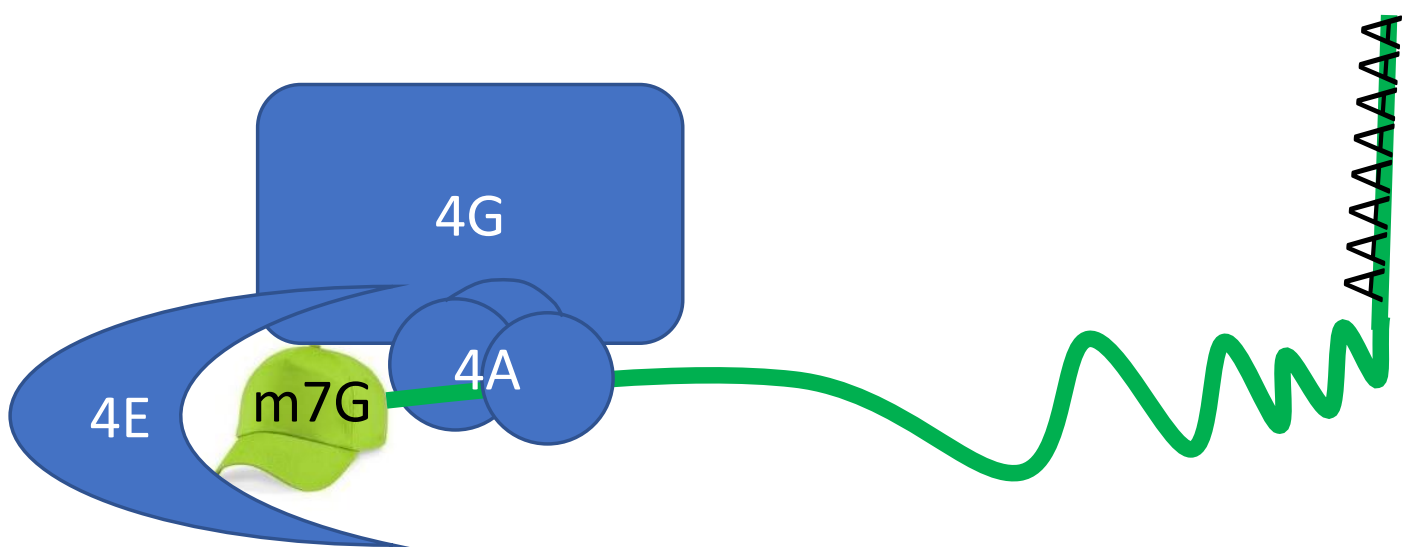

Figure 1-1 A simplistic diagrammatic representation of the elF4F complex engaged in cap-binding. In green, a messenger RNA possessing both a methyl guanosine ( $m 7 G$ ) cap and a poly-A tail is shown, elF4A the dumb-bell shaped protein, has assisted in melting secondary structure allowing the 40 S ribosomal subunit (not shown) to be recruited. Also shown is elF4G the scaffolding protein and elF4E the protein involved in cap-binding and recognition.

\subsubsection{Eukaryotic initiation factor $4 A$}

The eukaryotic initiation factor $4 \mathrm{~A}$ (elF4A), is a dumb-bell shaped protein with two major domains connected by a linker. It is an ATP-dependent DEAD-box RNA helicase of the eukaryotic initiation factor-4A family of proteins (Bordeleau et al., 2005). The two elF4A domains move between an open and closed conformation; in the closed conformation, the two domains interact in a way that favours the binding of mRNA and ATP (Sun et al., 2014). elF4A unwinds secondary structure in mRNA, and mRNAs with any secondary structure in the $5^{\prime}$ UTR ultimately require this activity for translation. The helicase activity of elF4A is increased as a by-product of stabilisation of the 'closed' conformation; the ATP binding pocket and mRNA binding region require both elF4A domains to interact to be fully form in the closed conformer, this becomes clear when viewing the binding pocket and $m$ RNA binding region in silico (Ho et al., 2009). The weak helicase activity of unbound elF4A increases 20fold when bound to the elF4G scaffold (Oberer, Marintchev, \& Wagner, 2005; Rozen et al., 1990). elF4A is vital because it is a requirement for $40 \mathrm{~S}$ ribosomal subunit binding to the mRNA for translation, and by extension, protein synthesis. Some mRNA molecules are ready to be translated into protein with little or no requirement for the action of elF4A. Other mRNAs have stable secondary structures in their $5^{\prime}$ untranslated regions (UTRs) like RNA duplexes, or even Gquadruplexes which require enzymatic activity to 'unwind'. The degree of requirement for the RNA helicase elF4A for translation is dependent on both the length and complexity of the transcript. Longer transcripts and those with greater secondary structure are more dependent on elF4A for translation (Svitkin, Pause, Haghighat, \& Pyronnet, 2001).

There are three isoforms of elF4A in humans: 4AI, 4All and 4AIII. 4AI and 4All share 90-95\% homology and in vitro assay work suggests they are functionally interchangeable (Yoder-Hill, Pause, Sonenberg, \& Merrick, 1993) but differentially expressed (Merrick, 1992). 4Al is expressed in all growing tissues while 4All preferentially binds to elF4G and is expressed in organs with low proliferative capacity (Nielsen \& Trachsel, 1988; Williams-Hill, Duncan, Nielsen, \& Tahara, 1997). $4 \mathrm{Alll}$ shares $65 \%$ homology with $4 \mathrm{Al}$ and $4 \mathrm{All}$ and is a core component of the exon junction complex 
involved in mRNA surveillance and nonsense mediated decay (Le Hir, Gatfield, Izaurralde, \& Moore, 2001). Due to its different function, elF4AIIl is not found in the elF4F complex.

\subsubsection{Eukaryotic initiation factor $4 \mathrm{E}$}

The role of the eukaryotic initiation factor $4 \mathrm{E}$ (eIF4E) component of the elF4F complex in eukaryotic translation initiation is to bind and recognise the $5^{\prime} \mathrm{m7G}$ cap, consisting of the sequence $\mathrm{m7GpppN}$ (where $\mathrm{N}$ is any nucleotide). There are three isoforms of elF4E in humans: 4EI, 4EII and 4EIII (Joshi, Lee, Maeder, \& Jagus, 2005). The isoforms differ in their ability to engage in cap-binding, with isoforms II and III being 40-fold weaker binders of the $\mathrm{m7G}$ cap (Frydryskova et al., 2016). elF4EI is the main isoform involved in global cap-dependent translation, elF4EII is involved in translational repression of a subset of mRNAs through AU-rich sequences in the 3' UTR and involving the protein Bicoid (Bcd) (Cho, Osler, \& Hg, 2008; Tao, 2015). Overexpression of elF4E is present in $30 \%$ of all cancers and is generally associated with a poor prognosis (Volpin et al., 2017). A hallmark of many cancers is over-expression of elF4E because unregulated growth increases demand for protein. In a given non-cancerous eukaryotic cell, protein synthesis is indirectly controlled by the stoichiometry of the elF4F components. Under normal conditions, the rate-limiting component of elF4F is elF4E, which is available at the lowest abundance in comparison to elAF4A and elF4G. The activity of elF4F is regulated through elF4E by $4 \mathrm{E}$ binding proteins (4EBPs), which in turn are regulated by hyperphosphorylation. The addition of multiple phosphate groups decreases the 4EBP binding affinity for elF4E, freeing it to interact with elF4G thus permitting cap-recognition to take place and allowing the elF4F complex to assemble. 4EBPs and eIF4G share a binding motif involved in elF4E binding, research shows that eIF4G and eIF4E interface at a single canonical alpha-helical motif (Zhao, Liu, Miller, \& Goss, 2017). The elF4E canonical binding motif has been characterised as Tyr-X$X-X-X-L e u-\phi$ where $X$ is any amino-acid and $\phi$ is a hydrophobic residue (Marcotrigiano, Gingras, Sonenberg, \& Burley, 1999). Awareness of this motif is important when considering how drugs target elF4E.

\subsubsection{Eukaryotic initiation factor $\mathbf{4 G}$}

The eukaryotic initiation factor $4 \mathrm{G}$ (elF4G) is the scaffolding protein and core of the elF4F complex. At least 2 genes for elF4G exist in humans, eIF4G1 and elF4G2. The N-terminal third binds to elF4E and poly-A binding proteins (PABPs), the middle third binds eIF4A and elF3, and the C-terminal third represents the regulatory portion, containing a second elF4A binding site and a docking sequence for the ser/thr kinase Mnk1 (Morino, Imataka, Svitkin, Pestova, \& Sonenberg, 2000). Mnk1 has been identified as the main kinase that acts on elF4E, and the phosphorylated form shows increased affinity for the m7G cap (Minich, Balastat, Gosst, \& Rhoads, 1994). Thus, elF4G has two elF4A binding sites, one in the central domain and one in the carboxylic acid terminal domain (Korneeva, Lamphear, Hennigan, Merrick, \& Rhoads, 2001). The association of elF4E with elF4G and is likely to cause conformational changes in the scaffold, modulating its capacity to interact with other elFs.

\subsection{4 elF4F inhibitors}

The elF4F complex has been identified as an excellent target for cancer therapy, as such an extensive number of drugs have been identified or synthesised that target components of the complex. Although the impetus for identifying elF4F inhibitors came from the fields interested in cancer therapy, inhibitors of the elF $4 \mathrm{~F}$ complex are also a useful tool to study the functional consequences of disturbing the translation initiation machinery. Several compounds exist that interact with the elF4F machinery in various mechanistically distinct ways. Known elF4A inhibitors include hippuristanol, pateamine A, elatol, the rocaglate family drugs and include an elF4A aptamer (Cencic \& Pelletier, 2016; Iwasaki, Floor, \& Ingolia, 2016; James H. Matthews, Maass, Northcote, Atkinson, \& Teesdale-Spittle, 2013; Oguro, Ohtsu, Svitkin, Sonenberg, \& Nakamura, 2003; Peters et al., 2018). 
elF4E inhibitors include 3 commercially available inhibitors, 4EGI-1, 4E1RCat, and ribavirin. Two of these inhibitors, 4E1Rcat and ribavirin, are the subject of this research, as their effects have not been extensively studied in the context of translation. These two compounds are described in more detail below. A short review of other elF4F inhibitors and their mechanisms of inhibiting elF4F components, and by extension translational output is also included.

\subsubsection{4E1RCat}

4E1RCat, is a small molecule inhibitor of elF4E that functions by preventing $4 E B P$ from binding elF4E, as well as preventing elF4E-elF4G interaction. 4E1Rcat has provided evidence that targeting the elF4F machinery can have positive outcomes for cancer treatment. Mice bearing Pten ${ }^{+/-} \mathrm{E} \mu-\mathrm{Myc}$, and $\mathrm{Tsc2}^{+/-} \mathrm{E} \mu-\mathrm{Myc}$ lymphomas showed similar regression patterns when treated with a 4E1RCatdoxorubicin combination in comparison to rapamycin-doxorubicin combination therapy (Cencic et al., 2010). 4E1RCat had no effect on tumour regression as a standalone treatment, whereas rapamycin and doxorubicin were individually capable of causing moderate tumour regression. (Cencic et al., 2010). In silico modelling of the molecular interaction of 4E1RCat with elF4E suggests that the elF4G/4EBP binding pocket is occupied by 4E1RCat preventing it from associating with elF4G (Cencic et al., 2010). Disruption of the elF4E/elF4G interaction occurs at the canonical elF4E binding motif previously mentioned. This mechanism is unlike 4EGI-1 which increases the binding affinity of elF4E for 4EBP (Moerke et al., 2007), the effect of 4E1RCat prevents 4EBP from binding elF4E as well as preventing elF4E-elF4G interaction signifying two molecular interactions to be aware of.

\subsubsection{Ribavirin}

Ribavirin is an FDA approved drug of the nucleotide analogue variety, it is used primarily for the treatment of Hepatitis $C$ viral infection. Ribavirin in combination with radiation therapy or temozolomide has shown increased efficacy in cancer treatment, in comparison to either treatment alone. This was demonstrated by an in increase in the median survival of rats and mice with orthotopically implanted gliosarcoma, and stem-like glioma cells respectively (Volpin et al., 2017). Unlike 4E1RCat, which can be considered pharmacologically 'clean', ribavirin has a host of molecular interactions and despite being approved by the FDA in 1986 some of ribavirin's interactions are still debated. Even the ability of ribvarin to inhibit elF4E has been hotly contested, especially the notion that it is an m7G mimic (Kentsis et al., 2005; Kentsis, Topisirovic, Culjkovic, Shao, \& Borden, 2004; Westman et al., 2005; Yan, Svitkin, Lee, Bisaillon, \& Pelletier, 2005). In a letter to the editor Kentsis et al, defend ribavirin as an elF4E-cap interactor mimicking the $\mathrm{m7G}$ cap, citing the pleiotropic, concentration dependent effects of ribavirin and usage errors made in the papers contesting their findings. They also direct the attention to the low-micromolar dose range in which ribavirin has been shown to interact with elF4E, as well as questioning the validity of the use of lysates as a tool to study a process as complex and highly regulated as translation initiation (Kentsis et al., 2005)

\subsubsection{A brief mechanistic appraisal of other elF4F inhibitors}

The elF4F complex is well characterised, and many inhibitors of the individual components have been identified using high-throughput screens and affinity assays. As elF4E is the main elF4F component investigated in this thesis, this section aims to briefly introduce other inhibitors and their mechanisms of (inter)action to demonstrate the range of inhibitory compounds available to biologists studying elF4F. This section also aims to indicate the role and context of this research in the field.

Pateamine $A(P a t A)$ is perhaps the most well-known elF4A inhibitor, it has an $\mathrm{IC}_{50}$ in the lownanomolar range and is suspected to function by increasing the binding affinity of elF4A for mRNA, 'locking' it onto a transcript, and disrupting protein-protein interactions with elF4G, thereby 
preventing translation initiation (Low, Dang, Bhat, Romo, \& Liu, 2007). Hippuristanol, is a polyhydroxysteroid with an $\mathrm{IC}_{50}$ in the high nanomolar range, it has a mechanistically distinct mode of action from PatA whereby the allosteric binding of the drug prevents association of mRNA with both free and elF4F complex bound elF4A (Waldron, Raza, \& Le Quesne, 2018). Elatol is another natural product inhibitor of elF4AI. Elatol's IC $\mathrm{C}_{50}$ is in the low-micromolar range, it binds elF4AI with 2:1 (elatol:elF4A1) stoichiometry and has been identified as a specific inhibitor of ATP hydrolysis (Peters et al., 2018). The rocaglate family a class of cyclopenta[ $b$ ]benzofuran drugs including rocaglamide A (RocA), silvestrol and episilvestrol originate from Aglaia sp these compounds tend to have $\mathrm{IC}_{50}$ values in the mid-to-low nanomolar range. The mechanism of RocA interaction involves a binding interface created by the mRNA:elF4A complex (Iwasaki et al., 2019), with an initial increase in helicase activity, but ultimately leading to elF4A inhibition (Sadlish et al., 2014). Silvestrol and episilvestrol interact with elF4AI/II but do not interact with any other molecular targets, and all molecular effects are traceable to their interaction with elF4AI/II (Chambers et al., 2013). The use of RNA aptamers involved development of an elF4A aptamer that 'staples' the two domains of elF4A into a closed conformation preventing the conformational changes necessary for ATP hydrolysis and helicase activity (Oguro et al., 2003). Antisense oligonucleotides have been developed against the elF4E transcript preventing production of the elF4E subunit, and are in Phase $\mathrm{I} / \mathrm{II}$ clinical trials in combination with the chemotherapy drug, irinotecan (Duffy, Makarova-Rusher, \& Ulahannan, 2016).

\subsection{The Pateamine A Story}

Pateamine $A$ is an immunosuppressive, cytotoxic, and anti-fungal inhibitor of eukaryotic translation initiation (Kuznetsov et al., 2009; Low et al., 2005; Northcote, Blunt, \& Munro, 1991). It is a thiazolecontaining macrodiolide. This small molecule sits at the motivational core of this project as it has been identified as a potential treatment of cachexia, through inhibition of the function of the elF4F complex (Di Marco et al., 2012). PatA was isolated from Mycale sp. sponges found off west coast of New Zealand. In 1991, an initial report published a description of its 2-D structure and its cytotoxicity in human cell lines (Northcote et al., 1991). A simplified synthetic analogue des-methyl, des-amino Pateamine A (DMDA-PatA) (see Figure 1-2A) has been synthesized and shows near equal potency (Romo et al., 2004) and has been used as a tool to study the behaviour of PatA by proxy. As a demonstration of DMDA-PatA toxicity researchers compared the cytotoxicity of PatA with an existing cancer therapy, vinblastine (see Figure 1-2B). A notable feature is the striking uniformity of the $\mathrm{IC}_{50}$ values in cell lines responding to PatA, almost all clustering at $10 \mathrm{nM}$, with few exceptions (Kuznetsov et al., 2009). DMDA-PatA, and by extension PatA has proven to be insensitive to Pglycoprotein mediated efflux. This resistance to efflux suggests that these molecules are likely to be more effective therapies against multi-drug resistant cancers (Kuznetsov et al., 2009), than therapeutics sensitive to removal by cancers employing a drug efflux strategy. Significantly, there are currently no therapeutics in the clinic that target translation initiation through the elF4F complex, an exploitable target for cancer therapy (Pelletier, Graff, Ruggero, \& Sonenberg, 2015). If PatA made it through clinical trials it would be the first compound to enter this new therapeutic territory. 

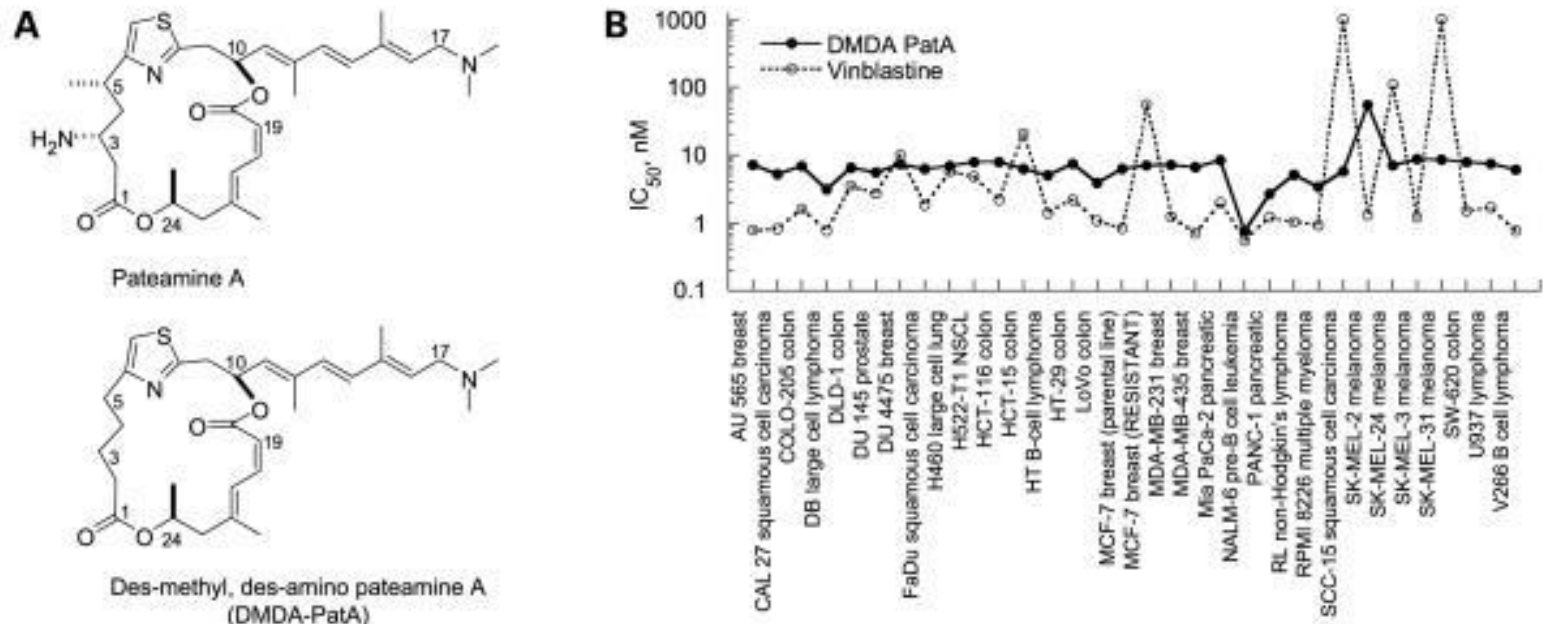

Figure 1-2 In growing cells DMDA-PatA (and by extension PatA) shows nearly uniform IC 50 values across 32 cell lines demonstrating comparable cytotoxicity to vinblastine an existing anti-neoplastic agent (Kuznetsov et al., 2009). The simplified synthetic analogue DMDA-PatA had previously been deemed equipotent with its natural counterpart pateamine A in an in vitro reporter assay (Romo et al., 2004). Reprinted from Molecular Cancer Therapeutics, 2009, 8(5), 1250-60, Galina Kuznetsov, Potent in vitro and in vivo anticancer activities of des-methyl, des-amino pateamine A, a synthetic analogue of marine natural product pateamine $A$ with permission from AACR.

\subsubsection{Therapeutic window of Pateamine}

Cancer therapies are infamous for having a raft of unpleasant, severe, and debilitating side-effects. This drives the biomedical field to search for new therapeutics with not only the ability to kill a range of cancers, but also considers the side-effect profiles and tolerability of a therapeutic. Early on in PatA's discovery, researchers noted the contrast between toxic effects on cells under static growth conditions and those actively dividing (Northcote, Blunt, \& Munro, 1991). P388, a murine cell line under active growth conditions exhibited an $\mathrm{IC}_{50}$ of $0.15 \mathrm{ng} / \mathrm{mL}$ whereas at $90 \%$ confluence, under static growth conditions the BSC kidney epithelial cell line had a high tolerance to the cytotoxic effects of PatA evidenced by an $\mathrm{IC}_{50}$ of $300 \mathrm{ng} / \mathrm{mL}$. These outcomes were mirrored in more recent work with DMDA-PatA treated quiescent human fibroblasts, showing little to no toxic effects at the low nanomolar concentrations associated with its toxicity to cancer cells (Kuznetsov et al., 2009). These effects suggest that PatA would be tolerated by somatic cells while being incredibly cytotoxic to rapidly growing cells - on the order of 1,000-2,000 times by these early studies. On paper, this indicates an acceptable therapeutic window, which refers to the range of useable concentrations between a drugs' effectiveness as a therapy and unacceptable toxic side-effects. In vivo work in nude mice shows utility in the treatment of cancerous xenografts in MDA-MDB-435 melanoma regression was achieved in 9 of 10 mice in comparison to 7 of 10 in paclitaxel at day 44 of the study (Kuznetsov et al., 2009). At study closure at day 65 , half of the study's cohort of mice were tumor free compared with a fifth of paclitaxel treated mice. It should be noted that the concentration of drug administered for success in these xenografts was extremely close to an empirically determined maximum tolerable dose. Despite this, not all xenograft models showed such promising results, some showed modest tumor regression. In LOX melanoma, less durable tumor regression was observed in comparison to paclitaxel. Modest regression was also reported in the remaining three tumor models, DLD-1 human colon cancer model, H522-T1 human non-small cell lung cancer model, and the NALM- 6 leukemia model. Two other xenograft models, MiaPaca-2 pancreatic cancer and HT29 colon cancer, showed no significant response to therapy (Kuznetsov et al., 2009). This body of research indicates that pateamine $A$ is in many ways a promising treatment for select cancers. The translation of this therapy to human trials is uncertain; the anti-cancer effects being close to a maximum tolerable dose is a concern for use as a therapy. Whether pateamine $A$ finds a place in the 
therapeutic space, either as a stand-alone therapy, in combination with existing therapies or in another therapeutic domain entirely (e.g. treatment of cachexia), it is undoubtedly a fascinating natural product with plenty of insights still to yield.

\subsubsection{The problem: Pateamine availability}

Total synthesis of Pateamine A was reported in a 1998 article by Daniel Romo (Romo et al., 1998), citing the compound's unique structure, potent immunosuppressive effects, and high levels of cytotoxicity as key drivers of synthesis efforts. A few years later a near equipotent simplified analogue, DMDA-PatA was synthesised with 10 fewer steps in the chemical synthesis (Romo et al., 2004). Despite this, Pateamine A is not commercially available. Efforts to cultivate Mycale $s p$. Sponges for semi-commercial production of peloruside A, another Mycale sp. derived anti-neoplastic agent, were largely unsuccessful. This method of procuring marine natural products was never intended to be commercially feasible and was done on a small scale for research in what was described as an 'interim supply' of compounds (M. J. Page, Handley, Northcote, Cairney, \& Willan, 2011). Unfortunately, predation of these farmed Mycale sponges by the nudibranch Hoplodoris nodulosa meant that over the course of 6-7 years just over a $1.12 \mathrm{~g}$ peloruside $A$ was obtained (M. J. Page et al., 2011). Had the endeavour to obtain peloruside A from sponges been successful, it would be reasonable to believe this means of production could have been mirrored for pateamine $A$. There are significant efforts being made at Victoria University of Wellington by the Harvey and TeesdaleSpittle group to produce peloruside $A$ as well as pateamine $A$ using synthetic chemistry approaches (Hemi Cumming et al., 2016). There are also retrosynthetic approaches using bacterial gene clusters expressed in a lab-culturable bacterial species (Owen et al., 2015) similar enough to the bacterial symbiont in Mycale to recombinantly express PatA by the Owen group.

\subsubsection{What can Pateamine and elF4A inhibition teach us about cachexia}

The story of pateamine does not end at cancer and the applications do not stop at cancer therapy. Low dose $\left(20 \mu \mathrm{g} \mathrm{kg}^{-1}\right)$ treatment with pateamine $\mathrm{A}$ has shown to rescue muscle wasting in vivo in mice with both $\mathrm{C} 26$ tumour induced muscle wasting, and the tumour-free TNF- $\alpha /$ IFN- $\gamma$ model (Di Marco et al., 2012). More recent work has confirmed that elF4A inhibition by several compounds with varied mechanisms proves that these effects are the result of elF4A inhibition (Cramer et al., 2018). It was noticeable that low doses of PatA were still able to ameliorate the muscle wasting phenotype. These doses were well below concentrations where its anti-cancer effects tend to manifest, evidenced by no noticeable regression of the C26 tumours, suggesting it would also be well below the threshold for toxic effects. Although higher doses $\left(50 \mu \mathrm{g} \mathrm{kg}^{-1}\right)$ both ameliorated cachexia and decreased the size of the tumours (Di Marco et al., 2012). The pleiotropic, dosedependent effects alone hint at interesting biochemistry, with two effects mediated by treating the translation initiation complex at different dosage regimes. Perhaps more interesting from a clinical standpoint was the observation that at low doses there were no noticeable toxic manifestations of the compound on the treated animals. In the treatment of cachexia, a growing body of research has implicated factors including the cytokine IL- 6 and the inducible nitric oxide synthase (iNOS) as well as the loss of myogenin and MyoD mRNA as the causative agents for muscle wasting (Di Marco et al., 2012). More recently, the signal transducer and activator of transcription 3 (STAT3) protein, a transcription factor and upstream effector of cytokines IL-6, TNF- $\alpha$ and IFN- $\gamma$, has been shown to be translationally repressed by elF4A inhibition (Cramer et al., 2018). Cramer showed that that STAT3 protein levels are affected by elF4A inhibitors without an effect on STAT3 mRNA levels suggesting that the effect is happening at the level of translation. In vitro INF- $\gamma$ and TNF- $\alpha$ treatment of myotubes results in cytokine induced atrophy of the muscle fibres. The elF4A inhibtors, pateamine $A$ (PatA), hippuristanol, and silvestrol have all been shown to ameliorate these effects. New research has also added silvestrol and hippuristanol to the list of compounds affecting the iNOS/NO pathway 
cementing elF4A inhibition as a potential central therapeutic target in the rescue of cachexia (Cramer et al., 2018).

\subsubsection{How does elF4A inhibition ameliorate cachexia at the molecular level?}

Although PatA is known to interact with all three isoforms of elF4A (Bordeleau et al., 2005), PatA's anti-cancer and anti-cachectic effects are likely to be mediated primarily by its interaction with elF4AI. There is a 4:1 abundance ratio in comparison to the elF4All isoform suggesting that at least based on abundance, elF4Al is the main isoform (Yoder-Hill et al., 1993). Pateamine A encourages stabilisation of the favourable mRNA and ATP binding closed conformer, increasing helicase activity but preventing interaction with elF4G (Iwasaki et al., 2019). Mechanistically, the anti-cancer properties of PatA at concentrations that lead to cell death are due to generalised protein synthesis inhibition. The sensitivity of tumor cells to its cytotoxic effects is likely a function of the protein demands of tumour cells going through unregulated division and the observation that many oncogenic transcripts are reliant on elF4F for their efficient translation (Wolfe et al., 2015). Consequently, elF4F components are often upregulated in cancers. The effects of elF4A treatment can be direct, at the level of translation, whereby the mRNA level is unaltered, but the protein expression level is decreased. For example, as noted above STAT3 mRNA levels remain unaffected by elF4A inhibition. However, elF4A inhibition leads to a decrease in STAT3 and phospho-STAT3 protein in response to elF4A. This was evidence that eIF4A inhibition was affecting STAT3 at the level of translation. The effects can also be indirect. STAT3 is a transcription factor, with many targets including IL-6. It is notable that IL-6 mRNA expression is lower after elF4A inhibition. The decreased mRNA levels suggest that IL- 6 protein levels have been indirectly affected, because inhibiting the production of protein should theoretically have no immediate effects on mRNA levels. STAT3 is the likely culprit for this disturbance.

A mechanism for the seemingly selective process by which inhibiting elF4A lowers protein expression in a non-uniform manner has not been fully identified. Some relatively recent research proposes a model of stress granule-mediated translational repression as a mechanism by which elF4A inhibitors could remove specific mRNAs. This repression of translation by removal to stress granules in this model ultimately leads to reversal of cachexia (Di Marco et al., 2012). It has been established that the degree of secondary structure present in the 5' UTR is in direct proportion to the requirement for elF4A for translation (Svitkin et al., 2001). Eukaryotic initiation factors are capable of binding and scanning unstructured 5' UTRs however, even weak secondary structures enforce the requirement of the helicase activity of elF4A for translation (Pestova \& Kolupaeva, 2002). Di Marco implies that the therapeutic effects of elF4A inhibition on models of cachexia are the result of the amount of $5^{\prime}$ UTR secondary structure present in elF4A sensitive transcripts (Di Marco et al., 2012). In other words, that the amount of secondary structure in the 5' UTR is the defining feature of an elF4A-sensitive transcript. Our understanding of this system is evolving as new inhibitors of the system are developed and evaluated. Some of these are described in section 1.2.4, above. For example, recent research pertaining to the interactions of the rocaglates with elF4A have shifted the understanding from translation dependent on 5' UTR length and complexity, to one of sequence-specific binding (Iwasaki et al., 2016). By implication, other elF4A inhibitors that strengthen the ability of elF4A to bind mRNA may also be manifesting their effects on the proteome in a similar manner. Rocaglates have been shown to increase the affinity of the binding interaction of eIF4A to select for polypurine sequences present in the 5' UTR (Iwasaki et al., 2016). X-ray crystallography also showed that the formation of the mRNA-elF4A dimer is required for rocaglates to 'lock' the complex together (Iwasaki et al., 2019). PatA's interaction with mRNA and elF4A has not been confirmed but is suspected to share this mechanism with rocaglates. 


\subsubsection{Is all mRNA born equal in the eyes of elF4A?}

It is clear that at high concentrations of elF4A inhibitors, the reduction in availability of free elF4A impacts on transcripts based on the 5' UTR requirement for functional elF4A (Wolfe et al., 2015). However, at lower concentrations, such as used in cachexia control, (Di Marco et al., 2012), it is possible that the selective removal of STAT3 and iNOS protein expression are a consequence of complex enzyme kinetics. The formation of the enzyme-substrates complex between elF4A, ATP and mRNA are a requirement for rocaglate binding. In this sense rocaglates can be considered uncompetitive inhibitors of elF4A as they require the assembled mRNA-elF4A interface. The substrate preference of elF4A for different sequences of $m R N A$ is only beginning to be explored (Iwasaki et al., 2016). It is likely that elF4A inhibitors bind more effectively to some mRNA sequences than they do to others. For example, recent publications suggest that rocaglates may favour binding to elF4A-mRNA complexes at polypurine motifs. The field has not crystallised the characteristics of elF4A dependent transcripts or whether interfering with other components of the elF4F complex has the same effect. This preference for particular sequence motifs may arise through the strength of binding interaction of the inhibitor with the elF4A-mRNA complex, with some sequences offering optimal binding sites. Alternatively, processing time may be an important factor. The rate of translation could be highly variable across the range of mRNAs the elF4F system is responsible for translating. There is strong evidence that eIF4A responds differently to structurally differing mRNAs, including exclusively Poly-U containing, mRNAs containing a mixture of duplex and single stranded RNA, and mRNAs that are extensively duplexed. It was noted that the mRNA variety used had highly variable effects on the conformational dynamics of elF4A (Harms, Andreou, Gubaev, \& Klostermeier, 2014). Therefore, the time it takes for mRNA to be processed may be variable, and this could be a factor determining the seemingly selective effects of elF4A inhibition, as uncompetitive inhibition is impacted by the lifetime of the enzyme-substrate complex.

PatA binding has been shown to be either a very strong or irreversible inhibitor of the function of elF4A (James Henry Matthews, 2010). If PatA shares the other trait of rocaglates, namely the selectivity for polypurine motifs, this could also potentially explain the selectivity seen with elF4A inhibition. However, recent work shows that hippuristanol still rescues the muscle wasting seen with in vitro models of caxechia (Cramer et al., 2018). This is notable, as hippuristanol is an allosteric regulator of elF4A, and linked to a decreased affinity for mRNA (Cencic \& Pelletier, 2016; Lindqvist et al., 2008), in contrast to PatA and the rocaglates). This is seeming evidence against the model of selective effects of elF4A inhibition being a product of sequestering into SGs of mRNAs with specific 5' UTR sequences or motifs such as polypurine motifs, hairpin loops or G-quadruplexes. Recent research suggests that the formation of classical secondary structures by $(\mathrm{GGC})_{4}$ motifs is favoured in comparison to G-quadruplexes and the role that G-quadruplexes play in 5' UTR motif mediated translation initiation repression may have been overestimated (Waldron et al., 2018). Unpublished experiments done by Richard Little ( $\mathrm{R}$ Little 2018, personal communication, 11 October) at Victoria University of Wellington laboratories suggest that polypurine motifs may be an enriched sequence in PatA sensitive transcripts.

Pateamine $A$ and other elF4A inhibitors show potential for the treatment of cachexia. There is ample evidence that the inhibition of elF4A is responsible for the anti-cachectic outcomes' researchers see in vivo. Current models implicate a loss of specific transcripts including iNOS and STAT3, while anabolic, muscle-generating transcripts such as myogenin and MyoD are rescued. The studies creating links between elF4A inhibition and transcript loss or retention based on specific sequences and structural motifs are preliminary. Currently, nothing is known about the sequence selective effects of inhibiting other members of the elF4F complex, and therefore whether any other elF4F inhibitors should be considered for anti-cachectic drug development. The goal of this research is to 
capture the effects both direct and indirect of translation inhibition by a selection of elF4E inhibitory compounds. These effects on translation should manifest in the proteome as a change in protein expression when compared to a population of untreated control cells.

\section{Aims and objectives}

Cachexia is a debilitating and sometimes fatal condition without current therapeutics. Targeting elF4A with inhibitory drugs like Pateamine $A$ leads to a non-uniform drop in protein expression across the proteome. The goal of this study is to determine whether there is merit in targeting the components of elF4F individually. We aim to perturb the eIF4F system in a targeted manner to study the global proteomic effects of treatment. We ask if we can achieve the same proteomic outcomes seen with Pateamine A using inhibitors for the other constituents of the elF4F system: ribavirin and 4E1RCat.

The objectives of this research were:

1) To determine the effective inhibitor concentrations (e.g. IC $C_{50}, I C_{10}$ and $I C_{1}$ ) of two elF4E inhibitors, and a relevant control inhibitor, by pharmacologically challenging a model cell system.

2) To quantify proteomic effects, pharmacologically challenge cells with inhibitors of elF4E, and a control inhibitor, at concentrations determined in Objective 1, and extract, purify and analyse cellular protein abundance changes using LC-MS/MS.

3) To use gene ontologies and co-expression analysis to assess the cellular processes affected by elF4E inhibition, with the ultimate goal of generating insight pathways effected by low dose elF4E inhibitor treatment.

4) To identify any sequence motifs in the $5^{\prime}$ UTRs of proteins that change in response to elF4E inhibition, but that are not explained by the direct biological response to the treatment. Finally, to evaluate whether 5'UTR sequence motifs are equivalent to those that respond to low-dose elF4A treatment.

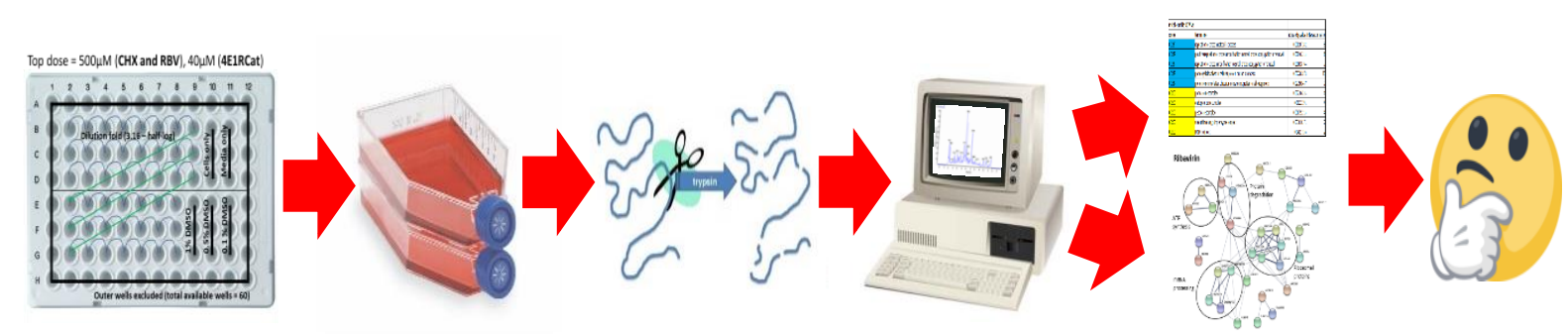

Figure 2-1 A simple diagrammatic representation of the work done in this thesis, from MTT assay through to treatment, tryptic digestion, LC-MS2 analysis and to gene ontology (GO) and STRING analysis. 


\section{Materials and methods}

\subsection{Reagents}

\subsubsection{Cell Culture}

96 well plates

Dulbecco's Phosphate-Buffered Saline (DPBS)

Foetal Bovine Serum (FBS)

Roswell Park Memorial Institute-1640 media (RPMI-1640)

HyClone $^{\mathrm{TM}}$

Dulbecco's Modified Eagle's Media (DMEM) High modified $+45000 \mathrm{mg} / \mathrm{L}$ glucose $+110 \mathrm{mg} / \mathrm{L}$ sodium pyruvate

T25 flasks

T75 flasks

Trypan Blue, $0.4 \%$

Trypsin-EDTA, $0.05 \%$

\subsubsection{Chemicals and Miscellaneous}

a-Cyano-4-hydroxycinnamic acid (CHCA), $99 \%$

Acetone, $>99 \%$

Acetic acid, glacial

Acetonitrile, $>99.9 \%$

Acetonitrile gradient grade for liquid chromatography

Bovine serum albumin (BSA)

Chloroform

Complete protease inhibitor cocktail

Dithiothreitol (DTT)

Dimethyl sulfoxide, $\geq 99.9 \%$ (DMSO)
Corning, USA

Life Technologies, NZ

Sigma-Aldrich, NZ

GE Life Sciences, USA

GE Life Sciences, USA

Corning, USA

Corning, USA

Life Technologies, NZ

Life Technologies, NZ

Sigma-Aldrich, NZ

Romil Ltd, UK

Merck, NZ

Carl Roth, NZ

Merck, NZ

ICP Biologicals, NZ

$\mathrm{BDH}, \mathrm{UK}$

Sigma-Aldrich, NZ

BioRad, NZ

Sigma-Aldrich, NZ 
Formic acid, $98-100 \%$

GlutaMAX ${ }^{\top \mathrm{M}}, 200 \mathrm{mM}$

lodoacetamide, >99\%

Kimwipes $^{\circledR}$

LoBind tubes ${ }^{\circledR}$

Methanol, $99.9 \%$

$\mathrm{N}, \mathrm{N}$-dimethylformamide (DMF), $\geq 99 \%$

Parafilm $\mathrm{M}^{\circledR}$

Sinapinic acid

Sodium deoxycholate (SDC), $\geq 97 \%$

Sodium dodecyl sulphate (SDS)

Thiourea, $99 \%$

Trifluoroacetic acid (TFA), $0.1 \%$ in $\mathrm{H}_{2} \mathrm{O}$

Triton-X-100

Trypsin, Mass Spectrometry Grade

Urea

$\mathrm{dH}_{2} \mathrm{O}$ - water used was purified by reverse osmosis

and distilled

ZipTip ${ }^{\circledR}$ pipette tips

\subsubsection{Kits}

DC ${ }^{\text {TM }}$ Protein Assay
Merck, NZ

ThermoFisher, NZ

GE Healthcare UK Limited

Kimberly-Clarke, USA

Eppendorf, GER

ThermoFisher, NZ

Sigma-Aldrich, NZ

Bemis, USA

Sigma-Aldrich, NZ

Sigma-Aldrich, NZ

Calbiochem, NZ

Merck, NZ

Sigma-Aldrich, NZ

ThermoFisher, NZ

ThermoFisher, NZ

Sigma-Aldrich, NZ

High-Q Inc. ${ }^{\mathrm{T} M}$, USA

Agilent Technologies, USA

Bio-Rad, USA

\subsubsection{Buffers and Solutions}

3-(4,5-dimethylthiazol-2-yl)-2,5-diphenyl tetrazolium bromide (MTT) solution: $5 \mathrm{mg} / \mathrm{mL}$ of $\mathrm{MTT}$, in PBS thoroughly vortexed and stored in a foil sheath. 
MTT solubiliser: $10 \%$ SDS: $50.0 \mathrm{~g}, 45 \% \mathrm{~N}, \mathrm{~N}$-dimethylformamide: $225.0 \mathrm{~mL}$, dilute to $500 \mathrm{~mL}$ with $\mathrm{dH} 2 \mathrm{O}, \mathrm{pH}$ adjusted to 4.5 with glacial acetic acid

Radioimmunoprecipitation assay (RIPA) lysis buffer: $25 \mathrm{mM}$ Tris, $\mathrm{pH} 7-8,150 \mathrm{mM} \mathrm{NaCl}, 0.1 \%$ sodium dodecyl sulfate (SDS), $0.5 \%$ sodium deoxycholate (SDC), $1 \%$ Triton X-100, protease inhibitor - RIPA buffer was made to $0.99 \mathrm{x}$ final volume, protease inhibitor tablets were dissolved to $0.01 \mathrm{x}$ in $\mathrm{dH}_{2} \mathrm{O}$ to produce a $10 x$ solution and combined to the produce the working solution.

Crude cell lysis buffer: $8 \mathrm{M}$ urea in $\mathrm{dH}_{2} \mathrm{O}$.

Urea SDC solution: $8 \mathrm{M}$ urea, $5 \%(\mathrm{w} / \mathrm{v}) \mathrm{SDC}$ in $\mathrm{dH}_{2} \mathrm{O}$.

Urea-thiourea lysis solution: $6 \mathrm{M}$ urea, $2 \mathrm{M}$ thiourea in $\mathrm{dH}_{2} \mathrm{O}$.

Alkylation buffer: $100 \mathrm{mM}$ iodoacetamide in $\mathrm{dH}_{2} \mathrm{O}$. Made fresh, tube covered in foil to minimise light exposure.

Reducing buffer: $10 \mathrm{mM} \mathrm{DTT}$ in $\mathrm{dH}_{2} \mathrm{O}$

Buffer $\mathrm{A}: 0.1 \%$ formic acid in $\mathrm{H}_{2} \mathrm{O}$ Optima ${ }^{\circledast}$ liquid chromatography/mass spectrometry.

Buffer B: $0.1 \%$ formic acid in acetonitrile.

CHCA matrix: $8 \mathrm{mg} / \mathrm{mL} \alpha$-Cyano-4-hydroxycinnamic acid (CHCA) in 50\% acetonitrile, $0.1 \%$ TFA in $\mathrm{H}_{2} \mathrm{O}$. Sinapinic acid matrix: $10 \mathrm{mg} / \mathrm{mL}$ sinapinic acid in 50\% acetonitrile, $0.1 \%$ TFA in $\mathrm{dH}_{2} \mathrm{O}$.

Trypan blue solution: $\mathrm{dH}_{2} \mathrm{O}, 0.4 \% \mathrm{w} / \mathrm{v}$ trypan blue

Trypsin solutions: $20 \mu \mathrm{g}$ trypsin of trypsin was resolubilised in $20 \mu \mathrm{L}$ of $\mathrm{dH}_{2} \mathrm{O}$ and split into $1 \mu \mathrm{g}$ aliquots

\subsection{Drug stocks (CHX, RBV and 4E1RCAT) - preparation and storage}

Ribavirin (RBV) and 4E1RCat were purchased from Sigma-Aldrich and stored at $-20^{\circ} \mathrm{C}$. Cycloheximide (CHX) was stored at $4{ }^{\circ} \mathrm{C}$. Drugs were dissolved in DMSO and stored at $-20^{\circ} \mathrm{C}$ at a concentration of 50 $\mathrm{mM}$ for RBV and CHX. 4E1RCAT was dissolved at $4 \mathrm{mM}$. Drugs diluted in aqueous solutions were used within 24 hours to avoid degradation. Drug stock tubes were wrapped in parafilm to avoid water adsorption, a well-known property of DMSO.

\subsection{Software}

Xcalibur $^{\mathrm{TM}}$

Scaffold ${ }^{\text {TM }} 4$
ThermoFisher Scientific, USA

Proteome Software, USA 
Proteome Discoverer ${ }^{\mathrm{TM}} 2.1$

Prism

Excel
ThermoFisher, NZ

GraphPad Software, USA

Microsoft, USA

\subsection{Cell culture}

\subsubsection{Cell culture - strains and maintenance conditions HL60 and HT29}

All cultures originated from existing liquid $\mathrm{N}_{2}$ freezer stocks at the Centre for Biodiscovery at Victoria University of Wellington. The HT29 cell line is derived from the colorectal adenocarcinoma of a 44year-old caucasian female, originally isolated in 1964 . The HT29 cell line has been used extensively in research, some examples of its use include its use as a measure of the effectiveness of antineoplastic compounds in drug discovery (Volpin et al., 2017) and to express genes due to its amenability to transfection (Morin, Vogelstein, \& Kinzlertt, 1996). The HL60 cell line is an acute promyelocytic leukemia derived line originating from a 36-year-old caucasian female. The HL60 cell line are well-used to study myeloid differentiation (Birnie, 1988), it is commonly used as a first step assessing a compounds toxicity against human cells in MTT assays in drug discovery at the Victoria University of Wellington. Cell cultures were maintained at $37^{\circ} \mathrm{C}$ in an incubator with a humidified atmosphere supplemented with $5 \% \mathrm{CO}_{2}$. HL60 Cells were seeded by default at a density of $1 \times 10^{5}$ cells $/ \mathrm{mL}, \mathrm{HT} 29$ cells were seeded at $2 \times 10^{5}$ cells $/ \mathrm{mL}$, cells were maintained in the exponential phase of growth and were kept from reaching confluency by regular passaging. Generally, 3 days passed between passages for both cell lines this was a good general rule.

\subsubsection{Cell culture media}

HT29 and HL60 cells were cultured in RPMI media containing 10\% (v/v) FBS and 2.05 mM L-

Glutamine. DMEM was required when thawing cells stored in DMEM or when growing cells from the stocks of another lab in which the cells were previously cultured in DMEM. In these cases the media was supplemented with 10\% (v/v) FBS and L-Glutamine, $2.05 \mathrm{mM}$. Cells grown in DMEM were transferred to RPMI and given at least one passage before they were used in any experiments.

\subsubsection{Cell counting}

Culture cell counts were performed using light microscopy and a $0.4 \%$ trypan blue solution to facilitate cell counting using a hemocytometer. The outer 4 and the middle squares in the grid were most commonly used. If cell counts were extremely consistent across 3 squares diagonally across the grid and were at enough numbers i.e. over 100 cells, then no more squares were counted. If cell numbers in a grid were below 50 , depending on the consistency of the first 5 squares counted, it was sometimes deemed necessary to count all 9 squares in the grid, or re-count a new set of cells.

\subsection{MTT cell proliferation assays}

For all MTT proliferation assays, 96-well microtiter plates were used. The wells of the 96-well plate were seeded at $1.0 \times 10^{4}$ cells $/ \mathrm{mL}$ for both $\mathrm{HL} 60$ and $\mathrm{HT} 29$ cells at a final volume of $100 \mu \mathrm{L}$. The effects DMSO has above $0.5 \%$ were taken into consideration when selecting the top dose in the MTT assays, due to a minor calculation error early in the project the top dose of all drugs corresponded to a $1 \%$ DMSO concentration. As an example, the top dose of CHX and RBV was $500 \mu \mathrm{M}$, whereas the top dose of $4 \mathrm{E} 1 \mathrm{RCat}$ was $40 \mu \mathrm{M}$. The two considerations when deriving the top dose for 4E1RCat were: the maximum achievable solubility in DMSO $(4 \mathrm{mM})$, and the maximum percentage of DMSO acceptable based on the literature. In HL60 cells a base proliferative decrease of $\sim 3 \%$ is noticeable at $0.5 \%$ DMSO. All outer wells were filled with sterile distilled water to avoid evaporation affecting the 
volume in the outer wells. Assays were generally spaced apart in terms of passaging. For example, cells were harvested from 3 separate passages from the same flask or the same origin cells over the course of 3 passages, instead of 3 biological replicate flasks being grown side by side and assays performed simultaneously. A half-log (3.16-fold) serial dilution scheme was used for all drugs (see Figure 6-1). A relevant DMSO control was included at concentrations where DMSO is expected to influence cell growth. A media only blank and cell-only control are also included. Assay data was collected from triplicate wells using a plate reader set at $570 \mathrm{~nm}$.

\subsubsection{HL60 cell line}

For the MTT assays with suspension cells the final well volume was made up to $100 \mu \mathrm{L}$. The media used to maintain cell lines was used to dilute the drug stocks in preparation for treatment. Drug preparation was done in the 96 -well microtiter plate prior to addition of cells. The top dose of drug was prepared in a single well if feasible (i.e. total volume does not exceed well volume of $300 \mu \mathrm{L}$ ). The well containing the top dose was split into three equal volumes among the triplicate wells, enough was prepared to leave $50 \mu \mathrm{L}$ in the wells of the top dose. The media-drug mix was moved between the remaining triplicate wells containing $50 \mu \mathrm{L}$ of media and mixed thoroughly to prepare a half-log (3.16-fold) serial dilution. Due to difficulty encountered culturing the HL60 cell line, the HT29 cell line became the focus of the proteomic analyses. Towards the end of this thesis the problems with the HL60 cell line was resolved, and a mirror experiment was carried out in this cell line, however, there was insufficient time remaining to accommodate analysing the HL60 samples on the MS.

\subsubsection{HT29 cell line}

Adherent cell lines were allowed 14 hours to adhere to the bottom of the MTT wells before treatment. Drug doses were prepared in a re-useable plastic container with a 96-well format, this was necessary for the HT29 cell line as the cells are allowed 14 hours to settle down in the 96 well plate prior to treatment. HL60 cells can have their drug doses prepared in a 96 well plate and have cells injected into the drug doses, this is possible as they are a suspension cell line. The top dose was made in a single well of the re-useable plastic container, then split into 3 equal volumes, enough liquid was prepared to have an excess of $50 \mu \mathrm{L}$ in the wells in the 96-well mimic container as it is difficult to fully recover all the liquid from the container. The top dose triplicate wells are used to construct the half-log dilutions down the length of the container, the media cells were cultured in was used to dilute the drug in each assay. After all the doses were constructed in the plastic container, they were transferred to the 96 -well plate and left for 2 days.

\subsubsection{Collecting dose response data}

The wells of a 96-well plates were seeded at $1.0 \times 10^{4}$ cells $/ \mathrm{mL}$ of HL60 and HT29 cells at a final volume of $100 \mu \mathrm{L}$. A $20 \mu \mathrm{L}$ aliquot of a $5 \mathrm{mg} / \mathrm{mL}$ MTT solution in PBS was pipetted into each well and the cells were given a 2-hour period to metabolise the MTT to formazan. After two hours the cells were lysed using the MTT solubiliser solution and left in an incubator overnight for the purple formazan crystals to dissolve. A (VERSAmax ${ }^{\mathrm{TM}}$ ) microplate reader set at $570 \mathrm{~nm}$ was used to read absorbance values. Excel was used to visualise general trends. Graphpad was used to generate graphs and get dose response values in the form of inhibitory concentration (IC) data. 


\subsection{Method of determining $I C_{10}$ and $I C_{1}$ from experimental data}

Two concentrations of drug were initially going to be explored, the $I C_{10}$ and the $I C_{1}$. For practical purposes and in the interest of time the $\mathrm{IC}_{10}$ was selected. These were determined experimentally by MTT assay. In Prism, graphs were generated from the absorbances expressed as a percentage of untreated controls. A non-linear regression was fitted for each MTT assay replicate. To avoid determining $\mathrm{IC}_{10}$ and $\mathrm{IC}_{1}$ by eye, $90 \%$ of the top asymptote value and $99 \%$ of the top asymptote values for each regression line on the graph were calculated and the corresponding $\log _{10}$ (drug concentration) determined for all replicates individually. These values were combined and averaged to give the expected drug concentration to achieve $10 \%$ inhibition and $1 \%$ inhibition respectively.

\subsection{Preparing cells for pharmacological challenge}

Biological replicates were grown to sufficient concentration to seed enough flasks at $2 \times 10^{6}$ cells. For example, HL60 cells were cultured to between $5-8.5 \times 10^{6}$ cells prior to treatment. T75 flasks were seeded at $2 \times 10^{6}$ and in the case of HL60 cells were treated immediately, whereas the HT29 cells are given 14 hours to adhere to the bottom of the flask. A 48-hour drug challenge was then applied to cells at IC 10 concentrations. HL60 and HT29 cells have a doubling time of about 24 hours under ideal conditions. The 48-hour treatment period was chosen to allow at least two mitotic divisions to occur. This treatment scheme was used to ensure the protein synthesis inhibitory effects of the drugs were given sufficient time to manifest. Inhibitory concentrations were determined by MTT proliferation assay described previously.

\subsection{Sample preparation for use in LC-MS/MS}

\subsubsection{Cell lysis}

Post-treatment cells were pelleted in a centrifuge at $300 \mathrm{xg}$ for four minutes. The media was decanted, and cells were subsequently kept on ice. For the remainder of the lysis steps the cells were also kept on ice. The cell pellet was resuspended in 5-10 mL of ice-cold PBS and re-pelleted at $300 \mathrm{xg}$ for a further four minutes, the PBS and all supernatant from this spin was discarded. Finally, $200 \mu \mathrm{L}$ of the $8 \mathrm{M}$ urea lysis solution was added to the cell pellet followed by vortexing. This combination of $8 \mathrm{M}$ urea and the cell pellet was flash frozen at $-80^{\circ} \mathrm{C}$ three times, between freezes the pellet was allowed to defrost, followed by vortexing. The resulting lysate was transferred to 1.5 $\mathrm{mL}$ Eppendorf tubes.

\subsubsection{Protein quantification}

Protein quantification was necessary to assess how much trypsin would be used for tryptic digestion. Trypsin was added at a ratio of 1:50 of trypsin to protein. Two methods of protein quantification were explored.

\subsubsection{NanoDrop (ThermoScientific)}

The protein extracts were resuspended in a $50 \mu \mathrm{L}$ solution of $8 \mathrm{M}$ urea and quantified using a NanoDrop spectrophotometer. An $8 \mathrm{M}$ urea blank was used to account for extraneous absorbance. $1 \mu \mathrm{L}$ of sample proteins was placed on the contact point, the other contact was lowered into place with the sample between the two. The protein quantification $A_{280}$ setting was used. Although the ratio of trypsin used to treat a given sample is 1:50 this can vary widely and still achieve successful digestion of proteins in a sample, however, the NanoDrop was exceptionally inaccurate with huge variation, even when analysing the same sample multiple times. The NanoDrop was considered too inaccurate for protein quantification and was dropped in favour of the DC protein assay. 


\subsubsection{DC DC $^{\mathrm{TM}}$ Protein Assay (Bio-Rad)}

Proteins were resuspended in $100 \mu \mathrm{L}$ of $8 \mathrm{M}$ urea or however much was necessary to fully redissolve the proteins. Proteins were quantified using the DC protein Assay (BioRad). Assays were performed in a 96-well microplate. A BSA standard was made at $40 \mathrm{mg} / \mathrm{mL}$ in $\mathrm{dH}_{2} \mathrm{O}(40 \mu \mathrm{g} / \mu \mathrm{L})$. Constructing a standard curve was achieved by performing 2 -fold dilutions; 12 dilutions was sufficient to reach the lower end of the detection capacity of the assay. For each well containing standard or sample liquids were added in the order: $25 \mu \mathrm{L}$ of reagent $\mathrm{A} \rightarrow 5 \mu \mathrm{L}$ sample/standard $\rightarrow$ $200 \mu \mathrm{L}$ reagent $\mathrm{B}$ (yellow). The colour developed within 15 minutes and was stable for an hour. The standard generated a linear correlation between absorbance at $750 \mathrm{~nm}$ and protein concentration between 200 and $1500 \mu \mathrm{g} / \mathrm{mL}$. Prism software from Graphpad was used to analyse the standard curve and sample data. For linear standard curves a simple linear regression function was fitted. A standard curve was generated with values outside the linear range, due to the small linear range of the assay this was extremely common. Due to the nature of tryptic digestion, often the amount of trypsin added is somewhat arbitrary and a 1:50 to 1:100 ratio of trypsin is more than sufficient to digest a protein sample.A sufficiently accurate result could be obtained by fitting a 2-phase decay non-linear regression to a standard curve starting at $40 \mu \mathrm{g} / \mathrm{uL}$ as the ratio of trypsin to protein will almost certainly be at a sufficient molar excess for digestion. Protein concentrations were interpolated based on the non-linear relationship between absorbance and protein concentration.

\subsubsection{Protein precipitation}

\section{Chloroform-methanol precipitation}

For every $100 \mu \mathrm{L}$ of protein containing sample supernatant, $400 \mu \mathrm{L}$ of methanol was added and agitated by vortexing. An aliquot of $100 \mu \mathrm{L}$ chloroform was subsequently added, followed by agitation. $300 \mu \mathrm{L} \mathrm{dH_{2 }} \mathrm{O}$ was added, agitated and the lysate-solvent mixture centrifuged at 13,000 G for two minutes. The aqueous phase containing chloroform was carefully removed, and a further $400 \mu \mathrm{L}$ of methanol added and vortexed. A second centrifugation pelleted the precipitated proteins. The remaining liquid was removed, and the protein precipitate was taken to near-dryness in the centrivap concentrator. Excessive drying caused issues with re-dissolving the protein pellet. The pellet was resuspended in an $8 \mathrm{M}$ urea solution.

\section{Acetone precipitation}

To the protein sample, four-times the sample volume of acetone cooled to a temperature of $-20^{\circ} \mathrm{C}$ was added. The protein-acetone solution was vortexed and incubated at $-20^{\circ} \mathrm{C}$ for one hour. The solution was subsequently centrifuged at $13,000 \mathrm{xg}$ for 10 minutes. The acetone was removed with a pipette with care being taken not to disturb the pellet, which was not always visible. The pellet was left to air-dry, then resuspended in $50 \mu \mathrm{L}$ of an $8 \mathrm{M}$ urea solution.

\subsubsection{Protein pre-treatment and tryptic digestion}

A $100 \mathrm{mM}$ dithiothreitol (DTT) solution was used to reduce cysteine residues, removing disulfide bridges, and give the protease used in subsequent steps free access to the lysine/arginine residues of the fully unfolded protein. DTT solution was applied to each Eppendorf-contained protein extract at a working concentration of $5 \mathrm{mM}$ and heated at $70{ }^{\circ} \mathrm{C}$ for 20 minutes. An iodoacetamide solution was added at $5 \mathrm{mM}$ to prevent disulfide bond formation by alkylating the reduced cysteines with carbamidomethyl groups. Sample urea concentration was lowered to $2 \mathrm{M}$ prior with $\mathrm{dH}_{2} \mathrm{O}$ prior to trypsin addition. Trypsin was added to sample proteins at a 1:50 ratio and left to digest overnight at $37^{\circ} \mathrm{C}$. 


\subsubsection{Concentration, purification and desalting of peptides}

Purification and desalting of the protein sample was achieved using a C-18 ZipTip ${ }^{\circledR}$. A selection of solutions was made that filled the roles of conditioning the ZipTip column, rinsing the ZipTip column, binding the peptides to the $\mathrm{C}-18$ resins, washing and desalting the peptides, and elution by a dilute solution of acetonitrile and neat acetonitrile.

Wetting solution $-100 \%$ acetonitrile

Cleaning solution $-\mathrm{dH}_{2} \mathrm{O}$

Sample solution - peptides contained in a $2 \mathrm{M}$ urea solution

Wash solution $-0.5 \%$ formic acid

$1^{\text {st }}$ Extraction solution $-0.5 \%$ formic acid in 1:1 $(\mathrm{v} / \mathrm{v})$ water: acetonitrile

$2^{\text {nd }}$ Extraction solution $-100 \%$ acetonitrile

Where possible, $100 \mu \mathrm{L}$ amounts of all solutions relating to the ZipTip procedure were used. During aspirating and dispensing of solutions the ZipTip was kept wet, and air was not allowed to enter the pipette tip. The wetting solution was aspirated and dispensed into waste 7-8 times. The cleaning solution was aspirated and dispensed into waste a minimum of 10 times to remove the acetonitrile wetting solution. The sample solution was aspirated and dispensed within a given sample tube a minimum of 10 times. The sample loaded on the pipette tip's C-18 resins was washed by aspirating and dispensing the wash solution into the waste 10 times. A fresh LoBind tube was used in the following steps, the first extraction solution was aspirated and left within the pipette for 20 seconds then dispensed into the fresh tube. This action was repeated with the second extraction solution. The extraction solutions were evaporated to near dryness, using the centrivap concentrator. Peptides were resuspended in $0.1 \%$ formic acid, transferred to a liquid chromatography sample tube and submitted to the LC-MS/MS for analysis.

\subsection{Matrix assisted laser desorption ionisation MS - time of flight (MALDI- TOF/MS)}

Early in the optimisation phase of preparing proteins for MS analysis the presence of large amounts of polyethylene glycol (PEG) was noticed. Polyethylene glycol has a suppressive effect on the ionisation of molecules in electrospray MS. With this in mind MALDI-TOF/MS was used on samples to assay for PEG prior to transfer to the mass spectrometer. The main reason that MALDI-TOF was used is because PEG can be detected immediately after protein precipitation from the raw cell lysate. Tryptic digestion and the ZipTip processing are time consuming and being able to assay for PEG was a time-saving approach. Additionally, since proteins were re-dissolved in $8 \mathrm{M}$ urea post precipitation and MALDI-TOF has a high salt tolerance it was a quick and easy way to detect PEG in samples and by a process of elimination identify the source of the PEG contamination. The Triton X100 used in the RIPA lysis buffer used at the outset of this project is the suspected source of PEG contamination.

\section{Matrix and MALDI}

For mass spectrometry analysis, $1 \mu \mathrm{L}$ of peptide-containing eluate was mixed with CHCA matrix in a 1:1 ratio and in a 1:10 ratio. Then $1 \mu \mathrm{L}$ of each elution-matrix mixture was spotted onto a 384 well AB SCIEX Opti-Tof ${ }^{\mathrm{TM}}$ Cal Mix 5 plate and allowed to dry. The AB SCIEX TOF/TOF ${ }^{\mathrm{TM}} 5800$ matrix-assisted laser desorption/ionisation time-of-flight tandem mass spectrometer (MALDI TOF/TOF) was calibrated using a 700-4000 m/z calibration mix. Many samples were in an $8 \mathrm{M}$ urea solution when placed on a spot on the 384 well plate. These samples had high concentrations of urea salts present on the spot and often required the maximal laser setting of 4000 units. 


\subsection{LC-MS/MS settings and data analysis parameters}

Peptides were separated using a flow rate of $0.2 \mu \mathrm{L} / \mathrm{min}$ and fractionated with a $\mathrm{C} 18$ column (Dionex, LC Packings, Netherlands). A 372 min buffer gradient was constructed from $0.1 \%$ formic acid (Buffer A) and $0.1 \%$ formic acid in $80 \%$ acetonitrile (Buffer B). The gradient was optimized to maximise the number of identified proteins using the gradient optimisation and analysis tool (GOAT) optimisation tool (Trudgian, Fischer, Guo, Kessler, \& Mirzaei, 2014). The peptides in solution were ionised by electron spray ionisation with a silica tip emitter, with the voltage potential set at 2.2 . Positive ion mode was used with the heated capillary temperature set at $200{ }^{\circ} \mathrm{C}$ and tube lens $160 \mathrm{~V}$ to permit entry of ions preferentially at $524.30 \mathrm{~m} / \mathrm{z}$. Ions with $\mathrm{m} / \mathrm{z}$ range between $200-1850 \mathrm{~m} / \mathrm{z}$ were analysed during the 372 min acquisition time using Fourier transform mass spectrometry (FTMS) in the Orbitrap, with data-dependent MS/MS on the top 6 intensity ions dynamically selected for collision-induced dissociation (CID) fragmentation and detection in the linear trap quadrupole (LTQ). The dynamic exclusion settings used were as follows: repeat count 1, repeat duration $30 \mathrm{~s}$; exclusion list size 500; exclusion duration $90 \mathrm{~s}$. A full-scan ( $500 \mathrm{~ms}$ maximum injection time) in the FTMS at a resolution of 30,000 identified the 6 highest abundance ions and selected them for CID (1.0 isolation width, normalised collision energy $35 \%$, activation $Q 0.25$, activation time $30 \mathrm{~ms}$ ) in the LTQ after accumulation of 500,000 ions with 10 ms maximum injection time. Between sample runs, the column was washed twice with a gradient from $2 \%$ Buffer B to $98 \%$ Buffer B across 30 min, the combination of washes used depended on how the chromatograms looked, if there was a lot of lipid at the end of the run a prolonged high \%B wash was used. A minimum of two technical replicates were carried out on each of 3 or more biological replicate samples.
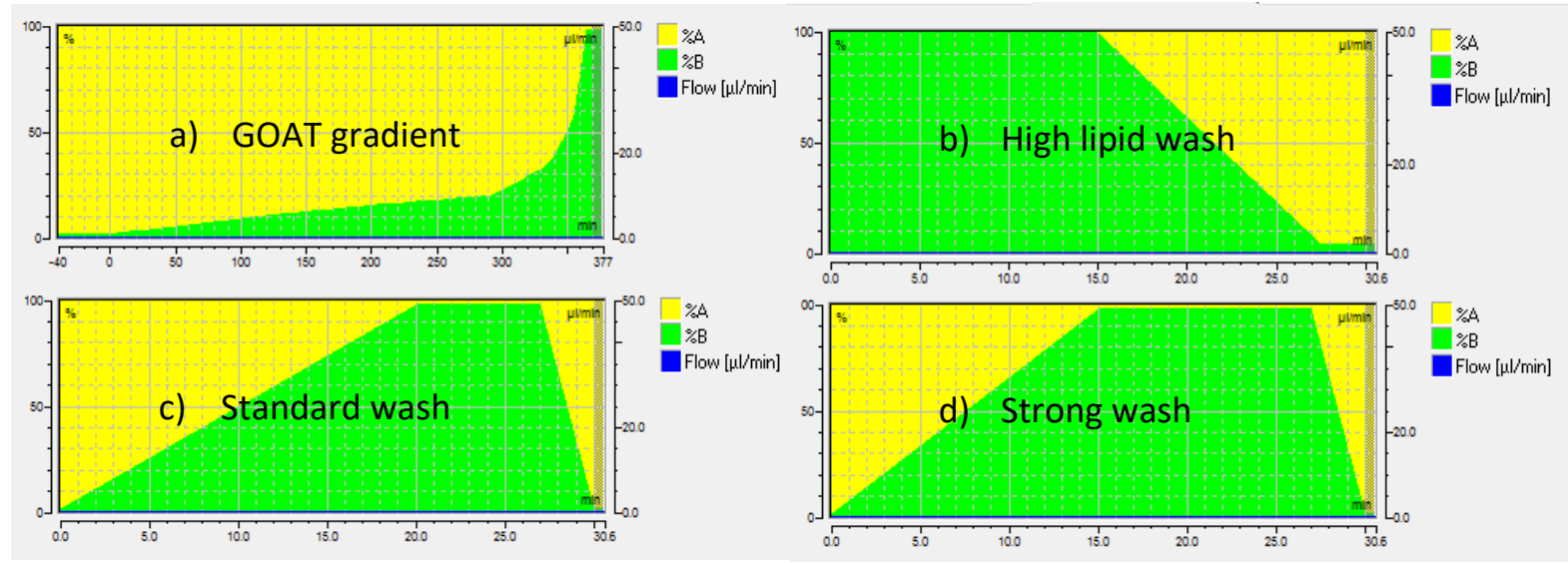

Figure 3-1 A selection of gradients used for LC-MS² analysis and column cleaning. The $100 \%$ max value on the $y$-axis corresponds to an $80 \%$ ACN solution. a) The GOAT gradient is an optimisation to spread the data evenly across a run. b) a high \%B wash employed in the final sample processing to ensure lipids were removed from the column prior to starting a new run. c) a standard linear wash gradient. d) A stronger wash gradient.

\subsubsection{Data analysis 1: Protein ID}

Mass spectra were analysed with both Sequest HT and Mascot search methods using Proteome Discoverer 2.1 (Thermofisher) against the entire Uniprot human proteome database (reviewed 07/09/2017). To aid in comparability between the data generated in this thesis and previous data collected by our lab group, an older Uniprot review was used. Parameters were set to a maximum of 2 missed cleavages with peptide lengths ranging from 5 to 144 amino acids selected. A precursor mass tolerance of $10 \mathrm{ppm}$ and fragment mass tolerance of $0.8 \mathrm{Da}$ were allowed. Modifications were as follows - Static: carbamidomethylation and dynamic: oxidation (methionine) and carbamylation (both $\mathrm{N}$-terminal and at lysine) 


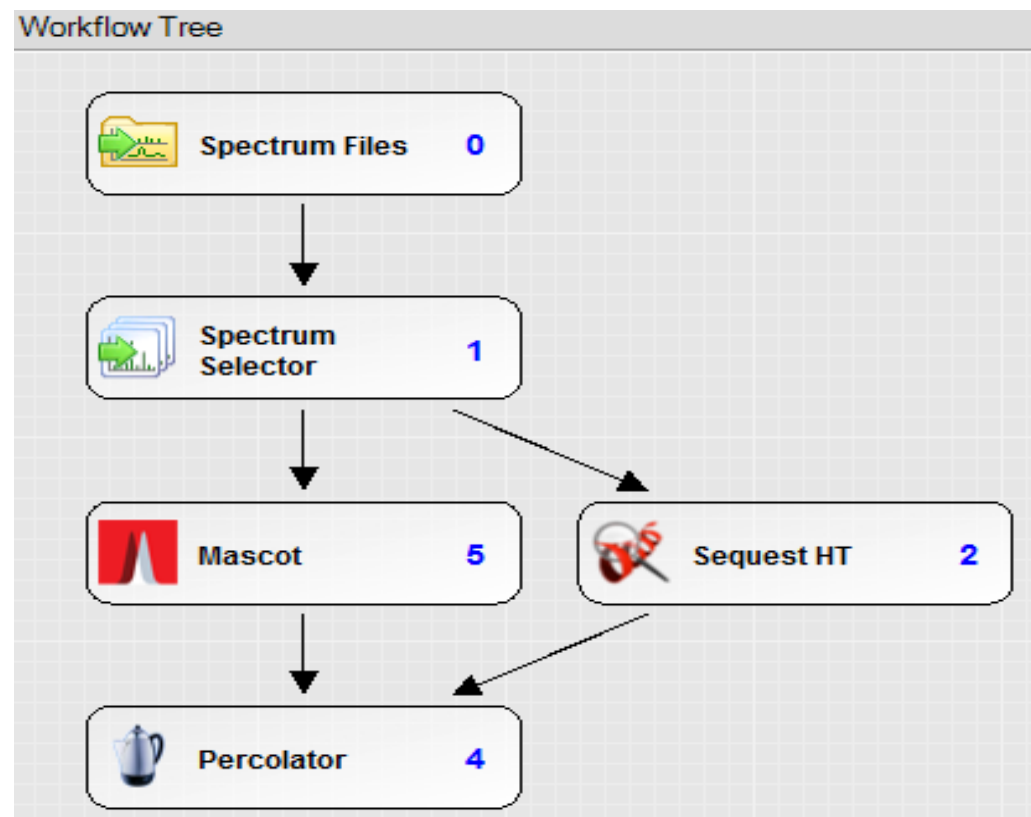

Figure 3-2 The Proteome Discoverer workflow showing sequence of nodes/modules used to analyse the RAW files from the LC-MS/MS. Static and dynamic modifications were set individually in Mascot and Sequest HT.

\subsubsection{Data analysis 2: Protein quantification}

Proteome Discoverer output files were then loaded into Scaffold $Q+(4.4 .8)$ proteomics software for quantification analysis. Technical replicates were combined using MuDPIT processing (Kislinger, Gramolini, MacLennan, \& Emili, 2005) and searches were again carried out against the Uniprot human proteome database (downloaded 07/08/2017). Protein abundance was quantified using the total TIC analysis method. Protein FDR thresholds were set to $1.0 \%$ and a peptide threshold of $0.1 \%$ FDR with proteins requiring at least 2 unique peptides to be identified. A protein abundance change, with a significance value less than 0.05 was considered significant.

The software converts the raw sequence files into potential peptide sequences. The peptide validator confirms whether the spectral data corresponds to a valid peptide. From this point, peptide sequences are matched against the Uniprot human proteome database for matches to defined protein sequences. The protein scorer assigns a quality score of the peptide sequence aligned with a known protein. Statistical due diligence from the FDR validator deals with false positives. 


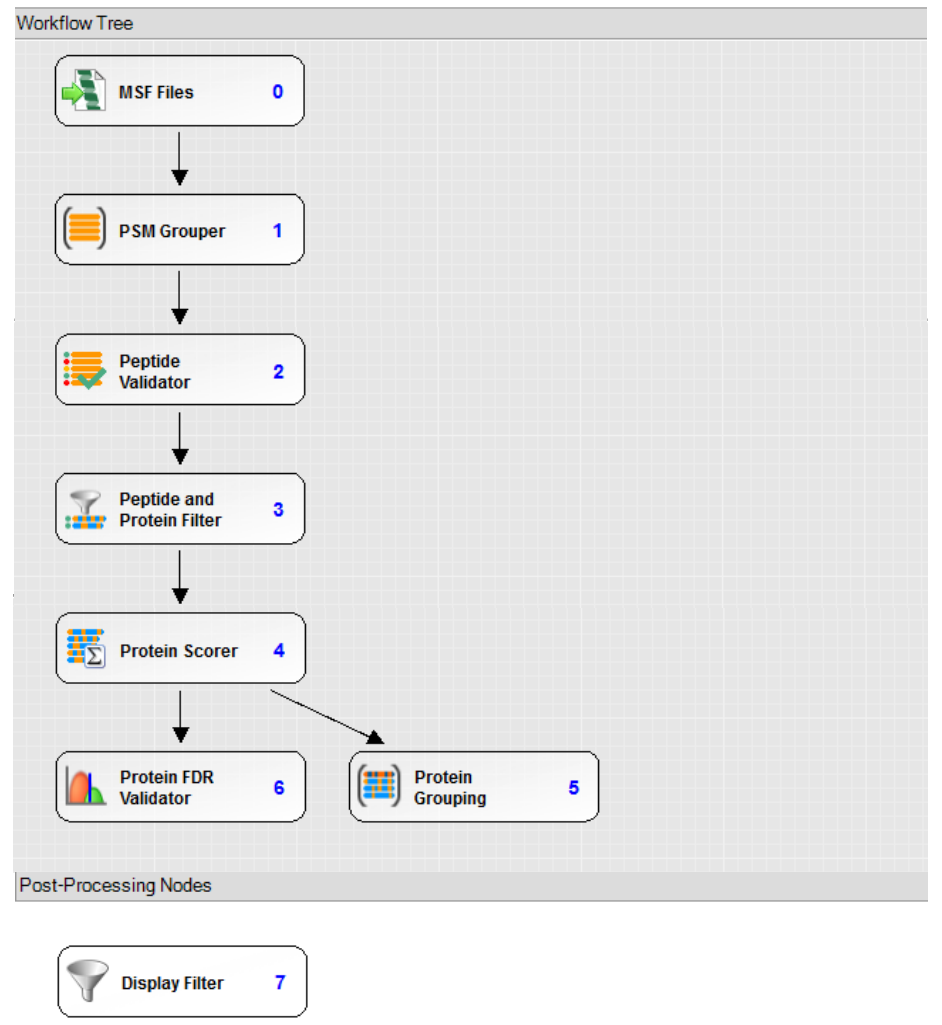

Figure 3-3 The processing workflow used to convert raw spectral data into peptide matches and ultimately to align sequences to known proteins to identify proteins from the spectral data. There are multiple nodes/modules used to perform statistics and validate findings

\subsubsection{Analysis of proteome responses}

Lists of proteins that changed in abundance to each treatment determined in Scaffold were compiled. The list of increasing, decreasing or combined change proteins were separately analysed for gene ontological (molecular function, cellular component, and biological process), pathway (KEGG and Reactome), and protein complexes (CORUM) enrichments through G:profiler (Reimand et al., 2016). Further analysis of clusters of proteins which had previously been found to be coexpressed was undertaken in STRING (Szklarczyk et al., 2019). 


\subsection{Gprofiler and STRING analysis pipeline}

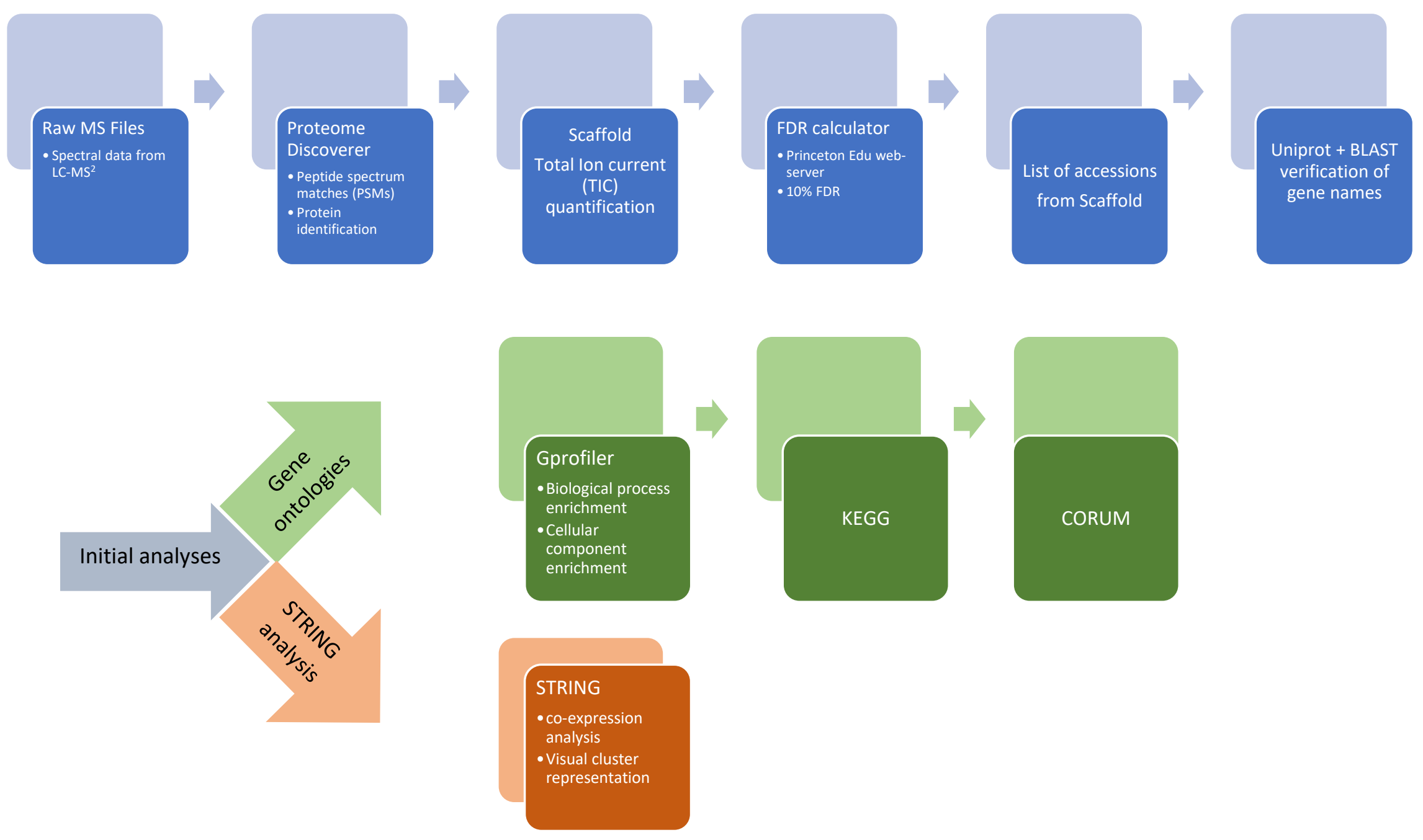




\section{Results}

\subsection{I $C_{10}$ and I $C_{1}$ treatment data}

One main objective of this project is to mimic the low-doses $\left(I C_{10}\right.$ and $\left.I C_{1}\right)$ of drug used in a previous proteomics experiments using pateamine A. Obtaining growth inhibitory concentration (IC) data was achieved via MTT assay and non-linear regression analyses on data displayed in Figure 4-1, Figure 4-2, Figure 4-3, Figure 4-4, Figure 4-5, Figure 4-6, Figure 4-7. The MTT assays for ribavirin and cycloheximide generate excellent dose response curves in both cell lines, a key difference is the HT29 cell line not completely ceasing metabolism in response to treatment with the curves reaching their asymptotes well before zero. The 4E1RCat assay data displayed for the HT29 cell line demonstrate the impracticality of using a compound with low solubility and antiproliferative capacity. Below is a table containing the $I C_{50}, I C_{10}$ and $I C_{1}$ data in HT29 and HL60 cells for the control protein synthesis inhibitor cycloheximide (CHX), the elF4E inhibitors ribavirin (RBV) and 4E1RCat as well as for the vehicle, dimethyl sulfoxide (DMSO) displayed in \% (v/v). A minimum of three biological replicates were used to obtain IC data for all drugs. The vehicle dose response data was done to ensure that the inhibitory effects of the vehicle were well established. For CHX and RBV treatment concentrations, the $\mathrm{IC}_{10}$ and $\mathrm{IC}_{1}$ values did not include the effects of DMSO as the vehicle is diluted to beneath biological significance. For 4E1RCat this is not the case; the $\mathrm{IC}_{10}$ concentration was hard to establish in both cell lines, in this case the top dose of $40 \mu \mathrm{M}$ was used as the $\mathrm{IC}_{10}$ concentration in both cell lines, indicated by $\mathrm{a} *$ in the table below.

Table 1 Growth inhibitory concentration (IC) data at 3 levels, the $I C_{10}$ values were the values used for further treatments. *4E1RCat: this compound was not sufficiently active to generate a full growth inhibition curve, and so IC values could not be modelled. The highest concentration used $(40 \mu \mathrm{M})$ gave a growth reduction equivalent to the $10 \%$ growth reduction seen with other treatments. Displayed in brackets beneath the IC vlaues are the $95 \%$ confidence intervals. Note that curve fitting was not possible with all of the data and in some cases it was not possible to generate IC values in Prism despite having curves that superficially appeared model-able.

\begin{tabular}{|c|c|c|c|c|c|c|}
\hline Drug & $\begin{array}{l}I C_{1} \\
H T 29\end{array}$ & HL6O & $\begin{array}{l}I C_{10} \\
\text { HT29 }\end{array}$ & HL60 & $\begin{array}{l}I C_{50} \\
H T 29\end{array}$ & HL60 \\
\hline Cycloheximide $(\mu \mathrm{M})$ & $\begin{array}{l}\mathbf{0 . 0 0 4} \\
(0.001 \text { to } \\
0.019)\end{array}$ & $\begin{array}{l}\mathbf{0 . 0 0 5} \\
(0.002 \text { to } \\
0.0178)\end{array}$ & $\begin{array}{l}\mathbf{0 . 0 3 8} \\
(0.020 \text { to } \\
0.071)\end{array}$ & $\begin{array}{l}\mathbf{0 . 0 4 8} \\
(0.025 \text { to } \\
0.088)\end{array}$ & $\begin{array}{l}\mathbf{0 . 3 0 5} \\
(0.226 \text { to } \\
0.412)\end{array}$ & $\begin{array}{l}\mathbf{0 . 3 4 9} \\
(0.259 \text { to } \\
0.471)\end{array}$ \\
\hline Ribavirin $(\mu \mathrm{M})$ & $\begin{array}{l}\text { Not } \\
\text { converged }\end{array}$ & $\begin{array}{l}\text { Not } \\
\text { converged }\end{array}$ & $\begin{array}{l}11.9 \\
(5.03 \text { to } \\
28.3)\end{array}$ & $\begin{array}{l}10.9 \\
(5.15 \text { to } \\
22.9)\end{array}$ & $\begin{array}{l}\mathbf{8 1 . 4} \\
(35.7 \text { to } \\
185)\end{array}$ & $\begin{array}{l}68.3 \\
(39.1 \text { to } \\
119)\end{array}$ \\
\hline 4E1RCat* $(\mu \mathrm{M})$ & $n / a$ & $n / a$ & $* 40$ & $* 40$ & $\mathrm{n} / \mathrm{a}$ & $\mathrm{n} / \mathrm{a}$ \\
\hline DMSO (\%) & $\begin{array}{l}\text { Not } \\
\text { converged }\end{array}$ & $\begin{array}{l}\mathbf{0 . 6 2 1} \\
(0.421 \text { to } \\
0.917)\end{array}$ & $\begin{array}{l}\mathbf{0 . 3 9 0} \\
(0.293 \text { to } \\
0.518)\end{array}$ & $\begin{array}{l}\mathbf{1 . 5 2} \\
(1.31 \text { to } \\
1.76)\end{array}$ & $\begin{array}{l}\mathbf{1 . 4 6} \\
(1.11 \text { to } \\
1.93)\end{array}$ & $\begin{array}{l}3.45 \\
(2.87 \text { to } \\
4.15)\end{array}$ \\
\hline
\end{tabular}




\section{Ribavirin effects on}

\section{HT-29 proliferation}

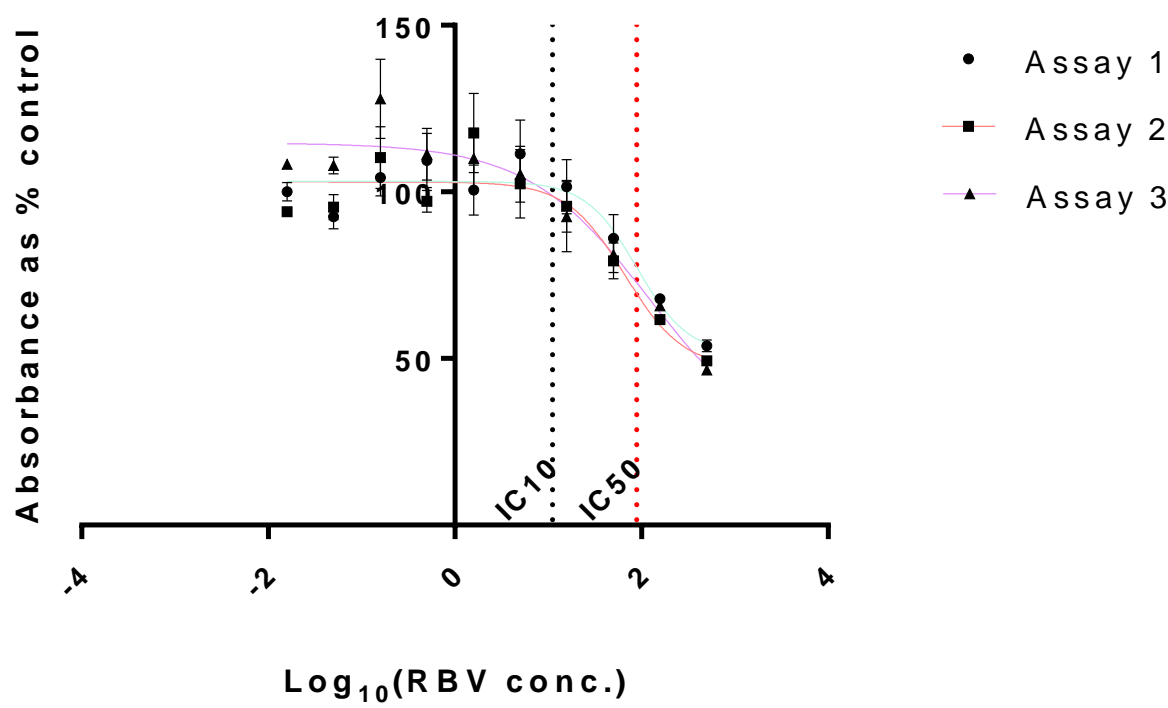

Figure 4-1 Absorbance changes reflect metabolic activity revealed by MTT treatment of $10^{5}$ cells treated with increasing concentrations of ribavirin, with maximum inhibitor concentration limited to comfortably within the limit of solubility, with no more than 1\% DMSO used in any dilution. Shown are the effects of ribavirin treatment on HT29 cell proliferation, graph generated in Prism. Note the failure to drop to total growth inhibition is not unusual in the HT29 cell line.

\section{Cycloheximide effects on \\ HT-29 proliferation}

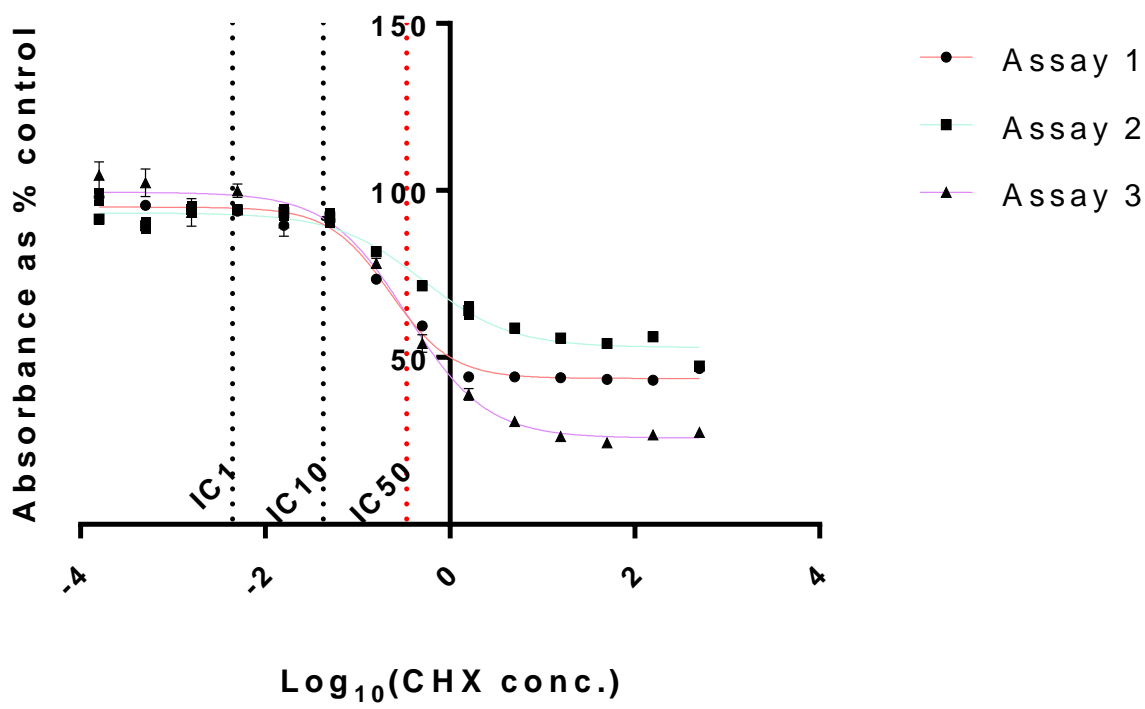

Figure 4-2 Absorbance changes reflect metabolic activity revealed by MTT treatment of $10^{5}$ cells treated with increasing concentrations of cycloheximide, no more than $1 \%$ DMSO was used in any dilution. Shown are the effects of cycloheximide treatment on HT29 cell, graph generated in Prism. 
4E1RCat effects on

HT-29 proliferation

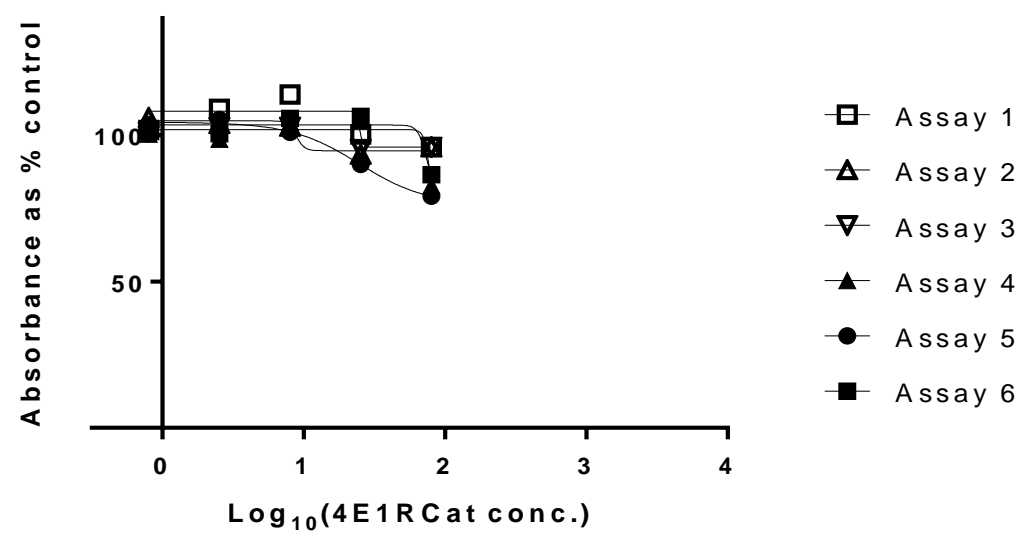

Figure 4-3 Absorbance changes reflect metabolic activity revealed by MTT treatment of $10^{5}$ cells treated with increasing concentrations of 4E1RCat, with maximum inhibitor concentration limited by 4E1RCat solubility, with no more than 1\% DMSO used in any dilution. Shown are the effects of 4E1RCat treatment on HT29 cell proliferation, graph generated in Prism. The DMSO vehicle at $1 \% \mathrm{v} / \mathrm{v}$ is likely to be partially contributing to the effects seen at the top dose of $40 \mu \mathrm{M}$.

Ribavirin effects on

HL-60 proliferation

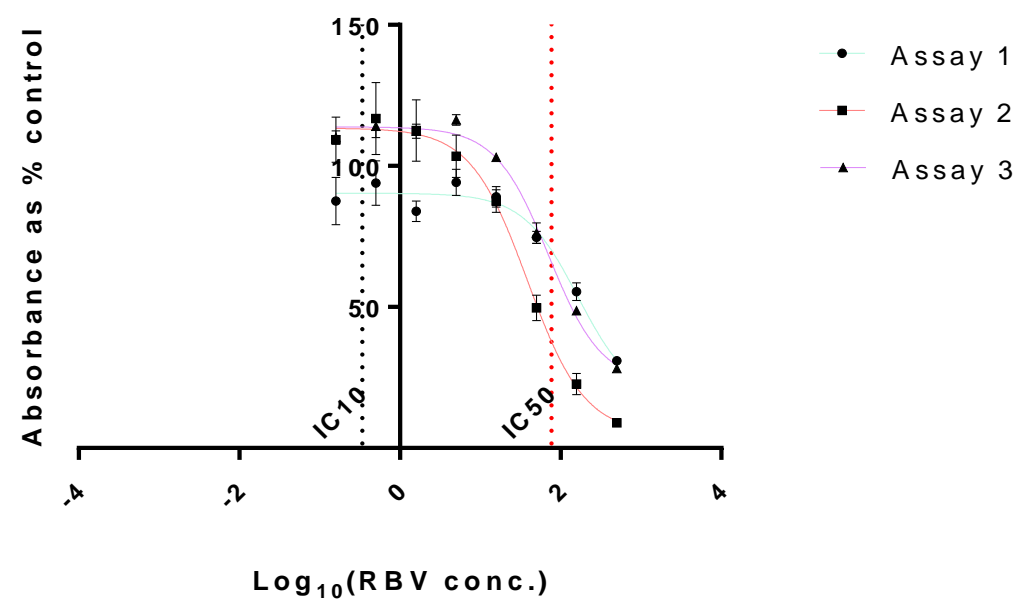

Figure 4-4 Absorbance changes reflect metabolic activity revealed by MTT treatment of $10^{5}$ cells treated with increasing concentrations of ribavirin, with maximum inhibitor concentration limited to comfortably within the limit of solubility, with no more than 1\% DMSO used in any dilution. Shown are the effects of ribavirin treatment on HL6O cell proliferation, graph generated in Prism. Note the drop to total growth inhibition is more noticeable in HL6O cells, presumably further increasing the ribavirin concentration would lead to total growth inhibition. 


\section{Cyclohexim ide effects on \\ HL -60 proliferation}

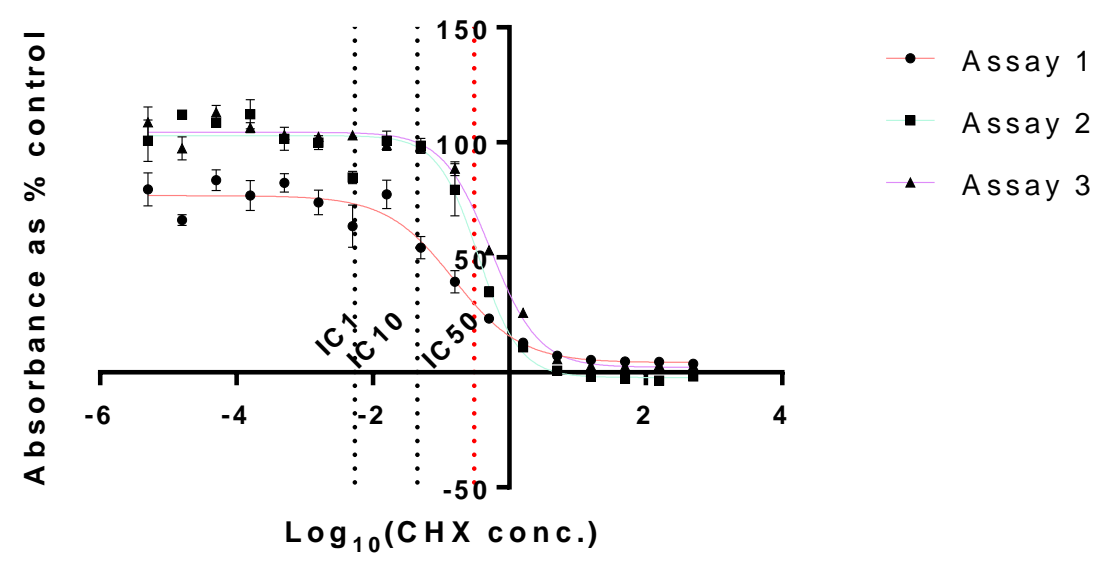

Figure 4-5 Absorbance changes reflect metabolic activity revealed by MTT treatment of $10^{5}$ cells treated with increasing concentrations of cycloheximide, no more than 1\% DMSO was used in any dilution. Shown are the effects of cycloheximide treatment on HL60 cell proliferation, graph generated in Prism. Note the drop to total growth inhibition.

\subsection{Effects of DMSO}

To establish the biologically relevant anti-proliferative effects of DMSO in the cell lines used in this project, MTT assays were used to generate a dose response between DMSO \% in solution and proliferation. These graphs are shown below. The minimum of 3 biological replicates rule was waived as this was exploratory and was done partly out of curiosity to ensure that the effects of the vehicle on cell lines was well established. This became useful when it became apparent that a calculation error had occurred that established that the top dose of each drug in the MTT assays corresponded to $1 \%$ DMSO which is slightly above the well-accepted $0.5 \%$ DMSO cut-off.

\section{DMSO effects on}

HT-29 proliferation

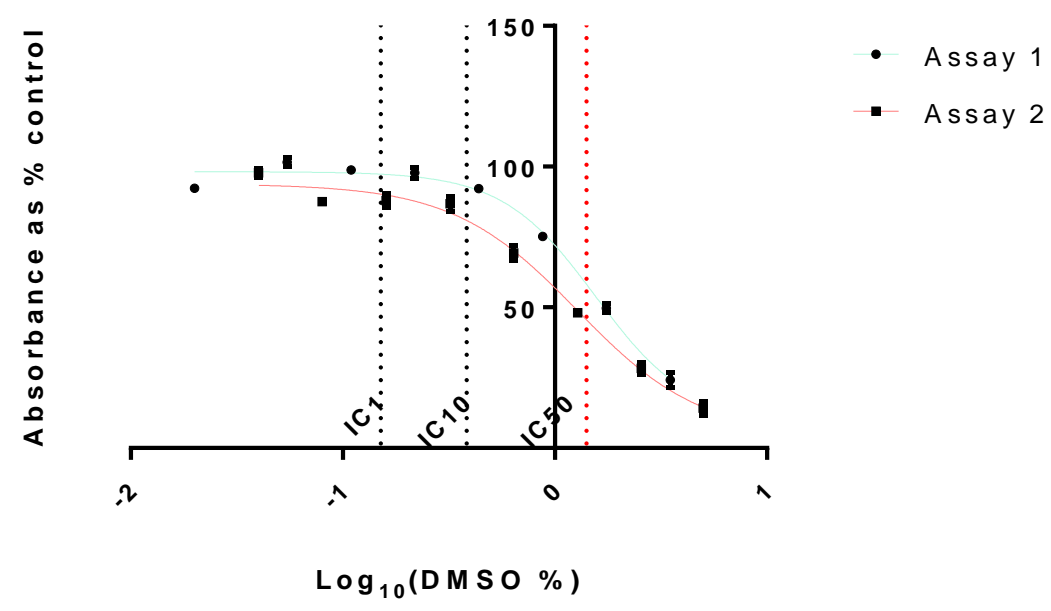

Figure 4-6 Effects of DMSO on HT29 cell proliferation, graph generated in Prism. 
DMSO effects on

HL-60 proliferation

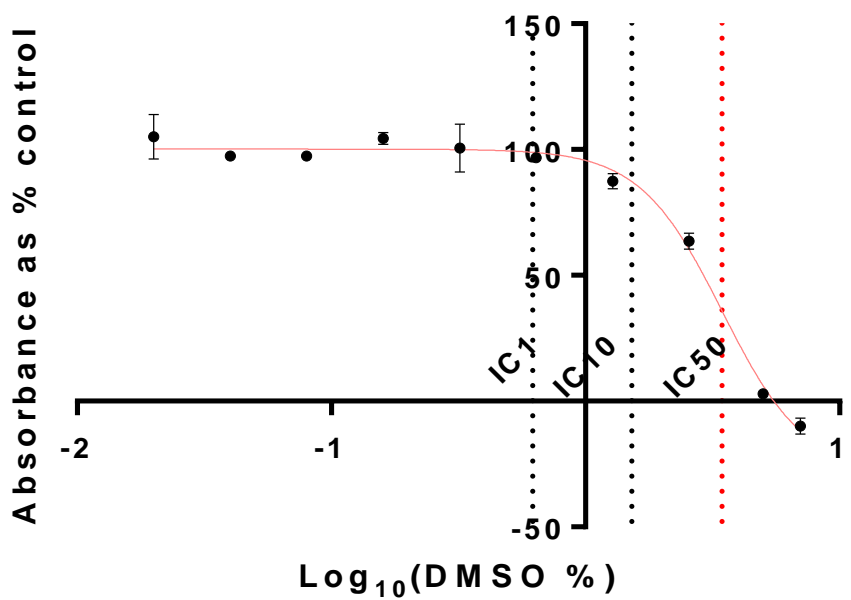

Figure 4-7 Effects of DMSO on HL60 cell proliferation, graph generated in Prism. 


\subsection{PEG contaminant detection by MALDI-TOF}

Polyethylene glycol (PEG) is a polyether compound with an array of uses from medicine to plastics to molecular biology. It is commonly used as a plasticiser to change the thermal and mechanical properties of plastics to increase their pliability. Among its other uses, PEG is used in non-ionic detergents, often chemically added to the detergent molecule to increase its capacity to interact with water. In the mass spectrometry setting, PEG has the capacity to suppress ionisation of an analyte by competing for charge and lowering the capacity to generate peptide fragments, thus lowering the sensitivity of the analysis. Early in the course of this thesis it was discovered that PEG was present in test samples run on the LC-MS. A set of experiments were conducted to determine the source of PEG contamination. There were two expected sources of PEG contamination, the plastics used over the course of the experiment, and the non-ionic detergent Triton X-100 present in the RIPA buffer. Matrix assisted laser desorption/ionization - time of flight (MALDI-TOF) was used briefly in this thesis to detect PEG contamination. Due to most of the protein samples being dissolved in urea, and containing high salt concentrations the use of the maximum laser ablation setting was often necessary. The goal of this analysis was to achieve spectra containing peptide peaks without the characteristic series of peaks seen with PEG. These peaks differ by 44 mass units, and strongly resemble a statistical representation of a normal distribution. Figure 4-8, Figure 4-9 demonstrate spectra with only matrix peaks from the CHCA and peptide peaks respectively, these are placed here to give the reader an idea of what to expect from a MALDI-TOF spectra. (Figure 4-10, Figure 4-11, Figure 4-15, Figure 4-16) were all from samples processed using RIPA buffer and were evidence that RIPA buffer was the likely source of PEG. This was confirmed by samples processed using urea demonstrated in several of the figures below (Figure 4-10, Figure 4-11, Figure 4-15, Figure 4-16), these samples are free of PEG contamination and have clean peptide peaks free from the intrusion of the visually distinct PEG spectral motif

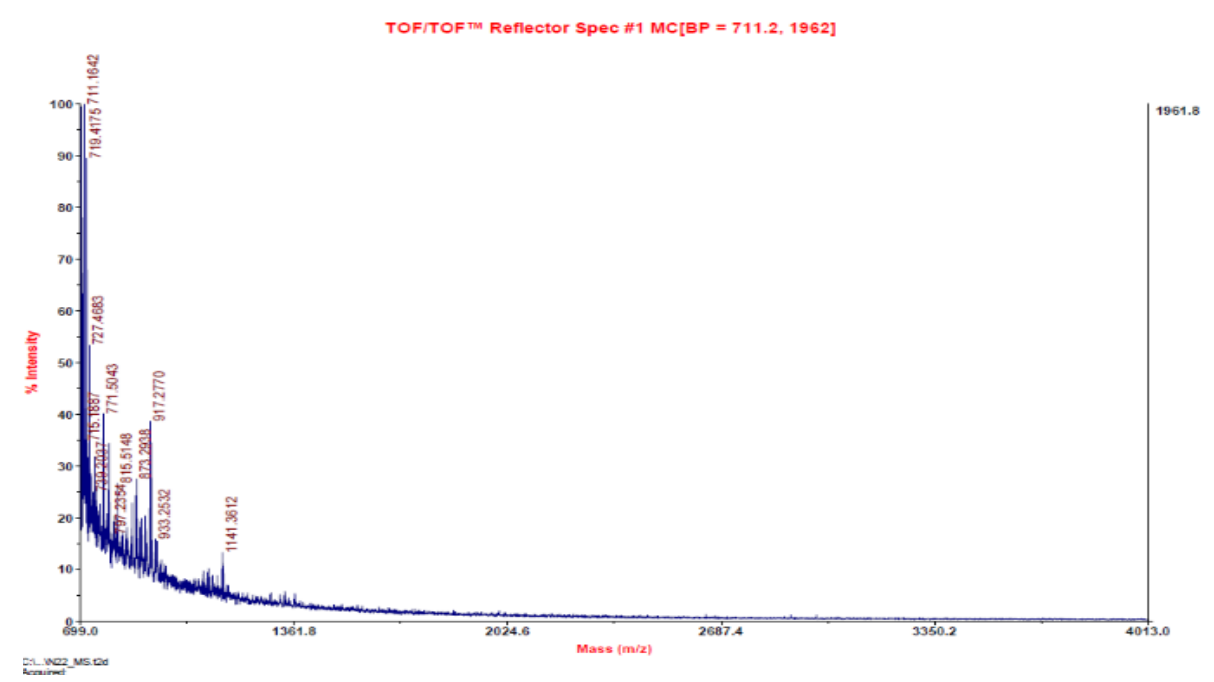

Figure 4-8 Spectra using maximum laser intensity where nothing of interest is visible, a few matrix peaks are visible near the $Y$-axis. 


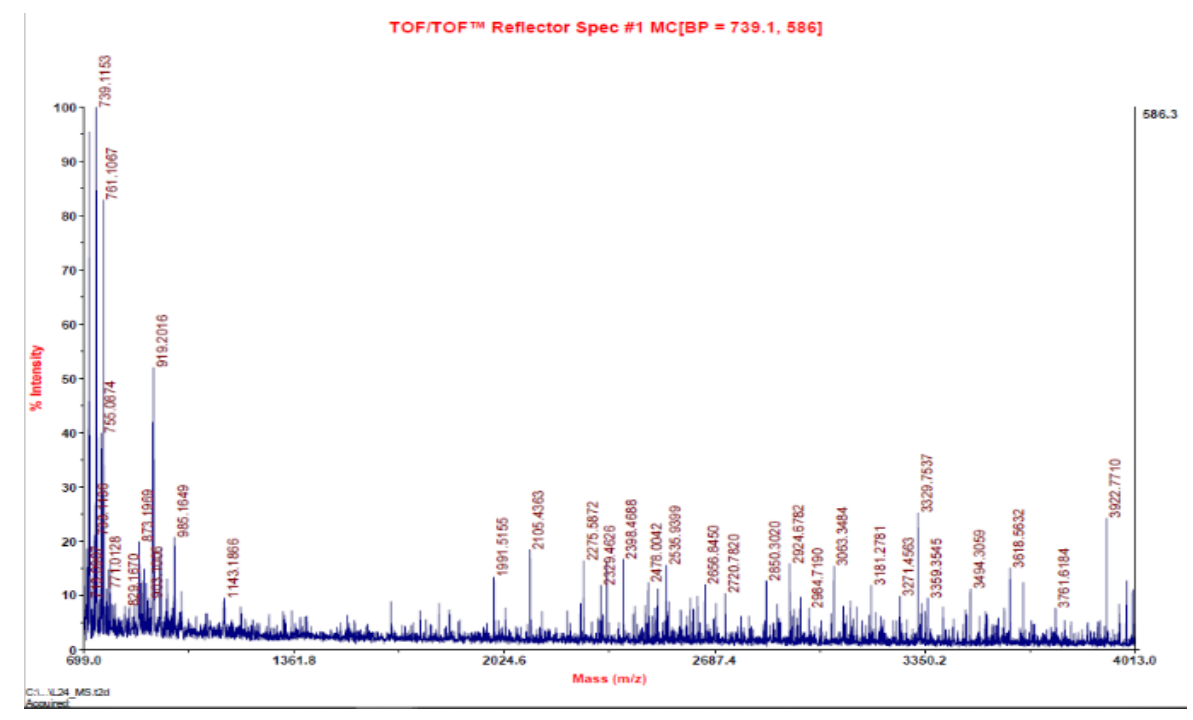

Figure 4-9 A spectrum showing matrix peaks near the $Y$-axis and demonstrating visible peptide peaks.

Pre-digestion, peptides in $2 \mathrm{M}$ urea were mixed with CHCA at a 1:10 ratio. Laser ablation at the maximum of 7000 units was used. There was a noticeable decrease in PEG between the two methods of precipitation (Figure 4-10, Figure 4-11), however still abundant at concentrations too high to run on the LC-MS.

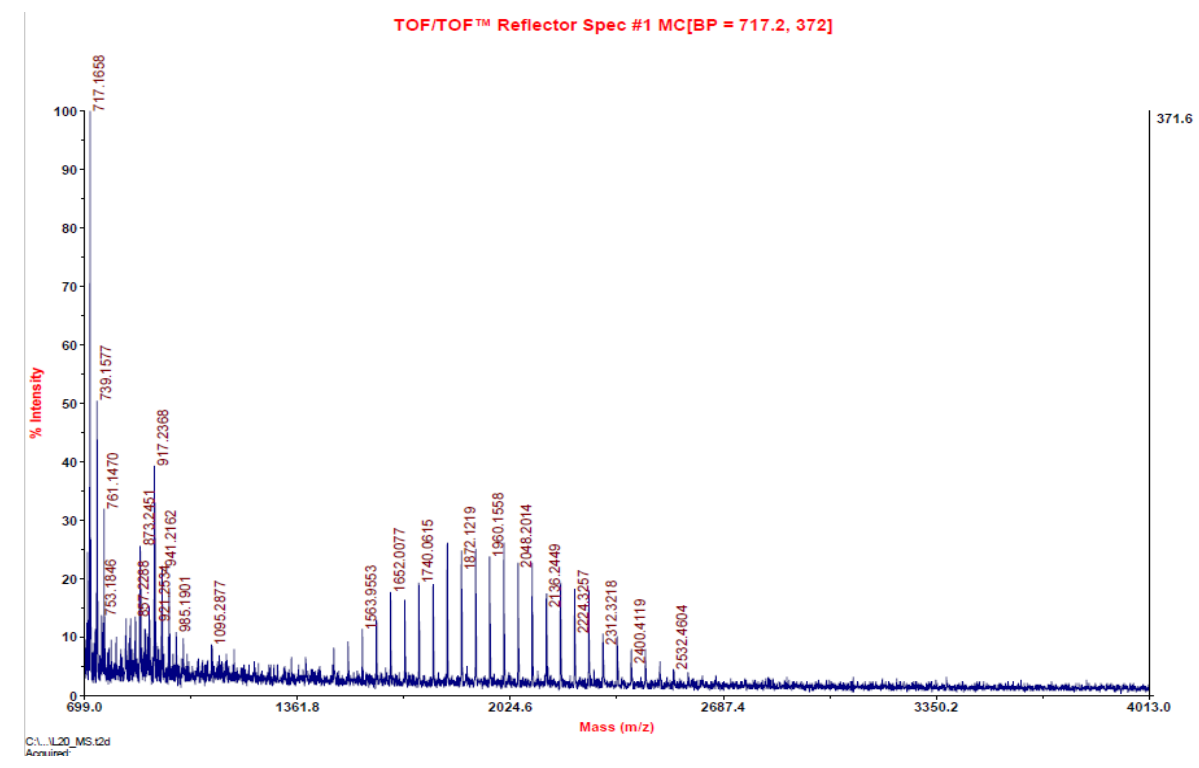

Figure 4-10 MALDI-MS/MS spectrum of a RIPA buffer precipitated PEG-contaminated sample following chloroform-methanol precipitation. 
TOF/TOF ${ }^{\mathrm{TM}}$ Reflector Spec \#1 MC[BP = 1917.1, 1213]

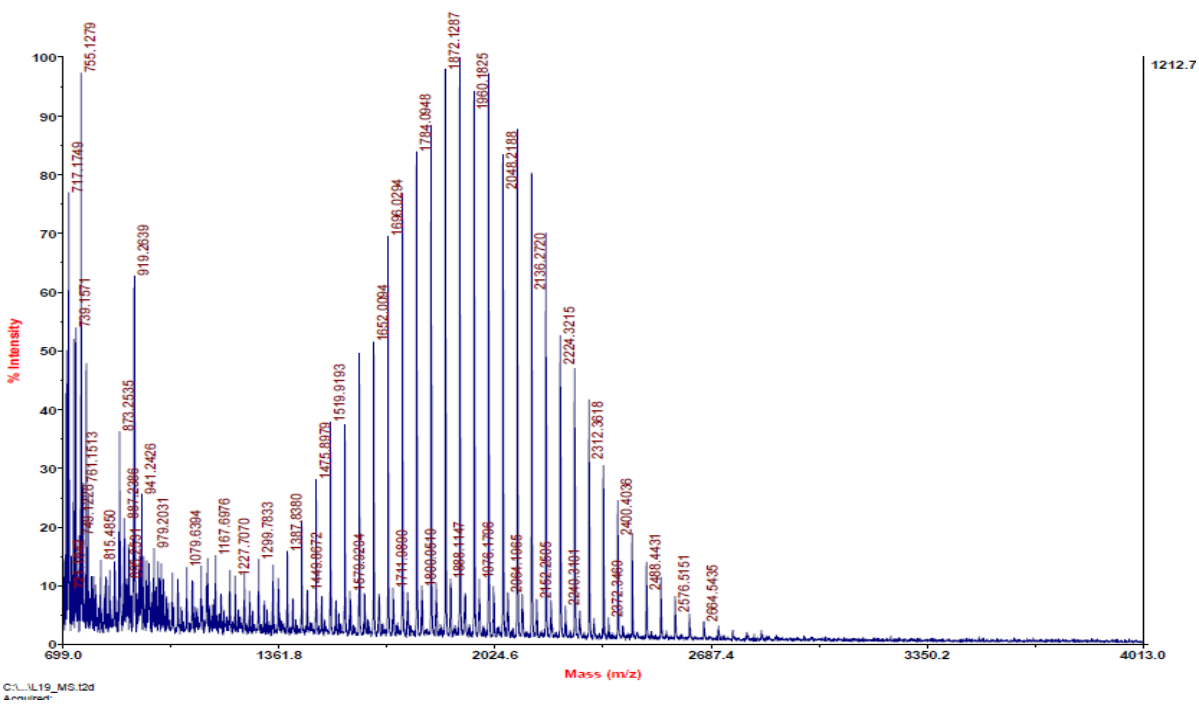

Figure 4-11 MALDI-MS/MS spectrum from a RIPA buffer precipitated sample showing particularly prominent PEG contamination in an acetone precipitated sample.

The figures below are post-digestion, ZipTipped peptides mixed with CHCA at a 1:10 ratio, Polyethylene glycol (PEG) is absent. They are a good demonstration of the spectrum expected from a complex protein sample without the presence of PEG.

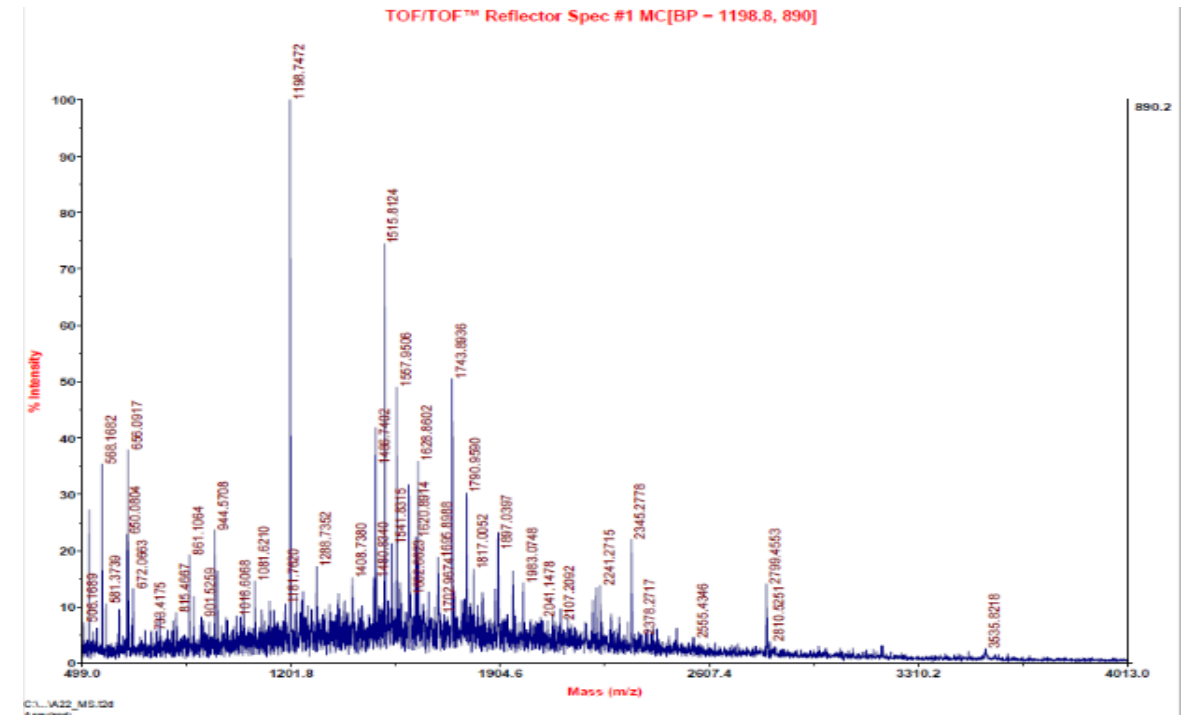

Figure 4-12 MALDI-MS/MS spectrum of a typical peptide sample (sample A22). 


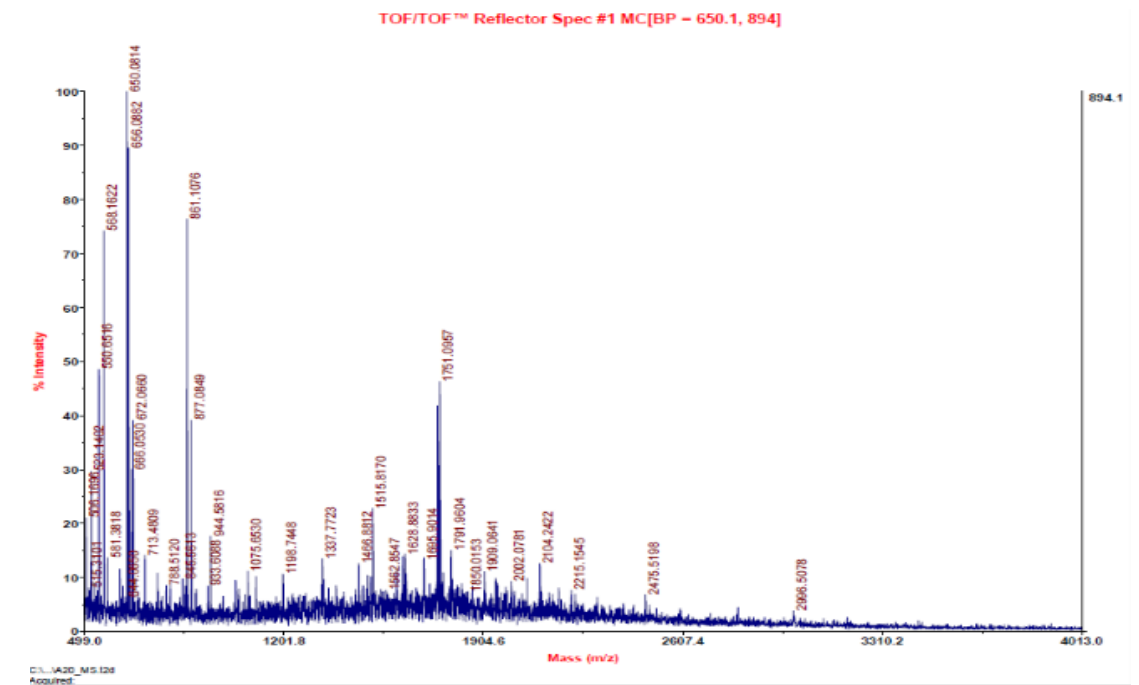

Figure 4-13 MALDI-MS/MS spectrum of a typical peptide sample (Sample A20).

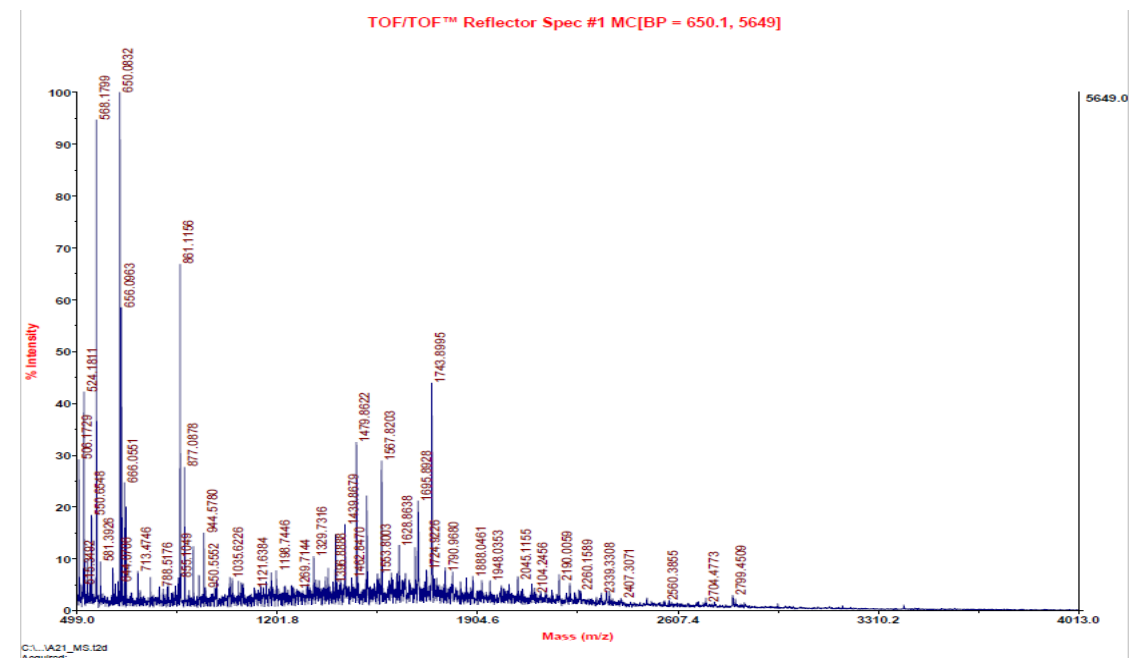

Figure 4-14 MALDI-MS/MS spectrum of a typical peptide sample (Sample A21).

The figures below show urea lysed, post-digestion, ZipTipped peptides mixed with CHCA at a 1:10 ratio. This is included as an example of polyethylene glycol that may come from another source, such as autoclaved pipette tips. Note the low abundance of PEG in comparison to peaks associated with the MS signal of the CHCA matrix on the left side of Figure 4-15. 


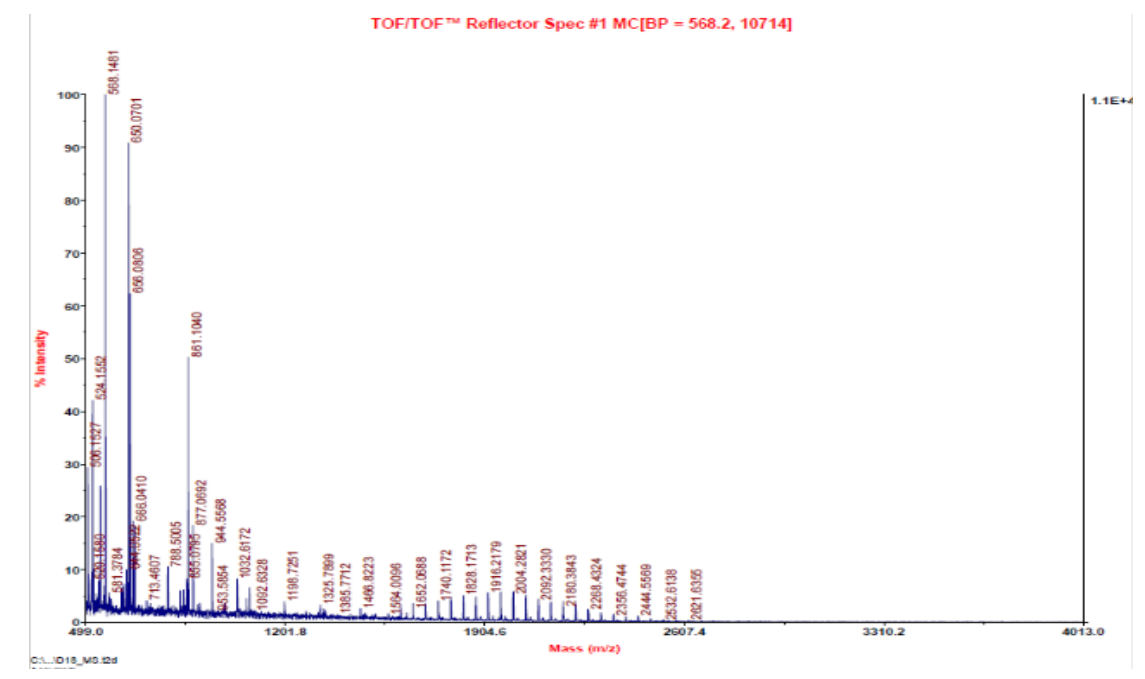

Figure 4-15 MALDI-MS/MS spectrum of a peptide sample (sample D18) with low peptide and PEG abundance in comparison to the matrix peaks on the far left.

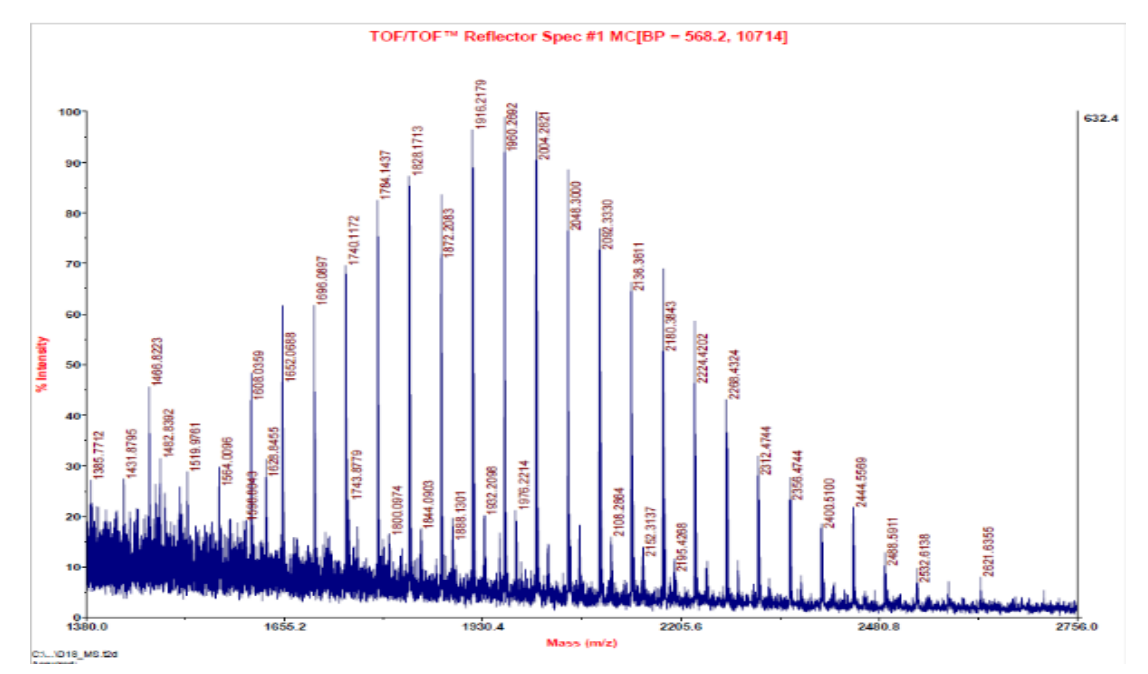

Figure 4-16 An expanded view of the PEG-containing region of the MALDI-MS/MS spectrum of sample D18.

\subsection{A side-by-side comparison of protein precipitation methods}

Side-by-side comparisons were made using the chloroform-methanol and acetone methods using known-protein standards (Bovine serum albumin) and HT29 raw-lysates to compare the efficiency and reproducibility of the two methods. The chloroform-methanol method produces an almost 'fluffy' pellet, which is useful for redissolving precipitated proteins. However, if sample proteins are not visible by eye, dislodging the pellet when removing the organic phase is a quick way to lose sample without realising it. As a result, although the chloroform-methanol method gave higher recovery in most cases, it occasionally lead to very low protein recovery. In contrast, the acetone precipitation method proved effective and robust and was adopted throughout this thesis. 


\subsection{Assessing the effectiveness of lysis solutions without non-ionic detergents}

A core component of this thesis was the DC Protein Assay, it was used extensively to quantify protein concentrations (Figure 4-17). Although the assay recommends working in the linear range of the assay, it was noted that the working range of the assay was inconveniently small, in response to this a larger range of concentrations were used and a non-linear curve fitted.

DC Protein Assay - Standard Curve using

Bovine Serum Album in

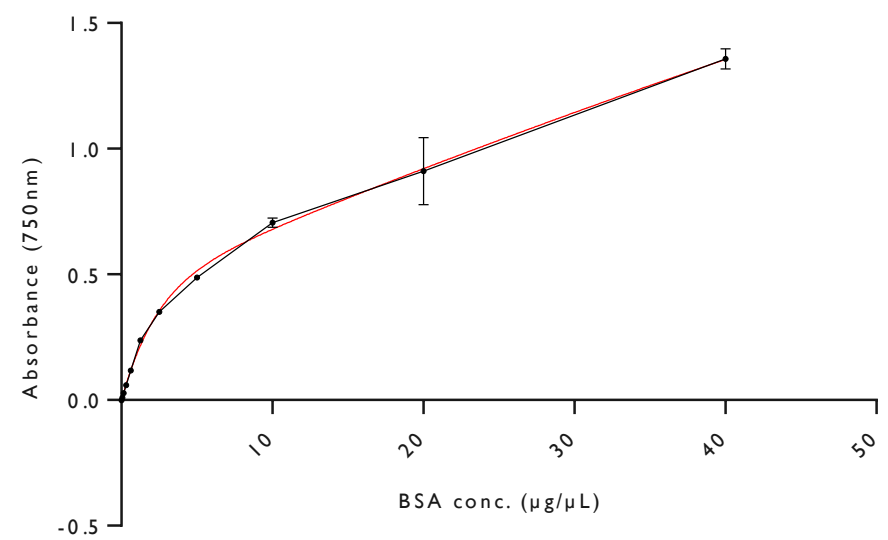

Figure 4-17 A standard curve of bovine serum albumin (BSA) was constructed using the DC protein assay, a portion of the graph between $200-1500 \mu \mathrm{g} / \mathrm{mL}$ has a direct linear relationship between absorbance and BSA concentration. A non-linear regression is fitted to the curve.

To eliminate PEG contamination alternative buffers without non-ionic detergent (e.g. Triton-X100, NP40) were explored for protein extraction efficiency. $1 \times 10^{6}$ cells were lysed using each buffer. Analysis was done using Prism, a non-linear 2-phase decay function was used to model the above standard curve to allow calculation of the protein concentrations in each sample.

Table 2 Lysis buffer versus protein concentration for several lysis methods. All buffers besides RIPA buffer were flash frozen at $-80{ }^{\circ} \mathrm{C}$ three times to aid in the lysis process.

\begin{tabular}{|c|c|c|c|}
\hline 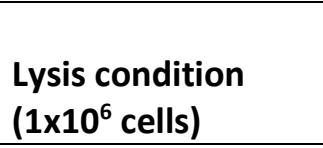 & $\begin{array}{l}\text { Protein } \\
\text { concentration } \\
(\mu \mathrm{g} / \mu \mathrm{L})\end{array}$ & $\begin{array}{l}\text { Efficiency relative } \\
\text { to RIPA buffer (\%) }\end{array}$ & $\begin{array}{l}\text { Total extracted } \\
\text { protein }(\mu \mathrm{g})\end{array}$ \\
\hline RIPA lysis buffer & 2.10 & 100 & 420 \\
\hline $8 \mathrm{M}$ urea & 1.39 & 66.2 & 278 \\
\hline $8 \mathrm{M}$ urea (replicate) & 1.36 & 64.8 & 272 \\
\hline $8 M$ urea $+5 \%$ SDC & 0.87 & 41.4 & 174 \\
\hline $\begin{array}{l}\mathrm{dH}_{2} \mathrm{O}+\text { protease } \\
\text { inhibitor }\end{array}$ & 0.45 & 21.4 & 90 \\
\hline
\end{tabular}

Although RIPA buffer was $\sim 1 / 3$ more effective than $8 \mathrm{M}$ urea, it was significantly better than $8 \mathrm{M}$ urea containing SDC and distilled water with protease inhibitor. 


\subsection{Mass spectrometry optimisation - chromatograms}

Early attempts to generate spectra were largely unsuccessful, it was noted that samples from the earliest attempts were very low in protein content, comparable to the spectra for unrelated samples shown in figures Figure 4-18 and Figure 4-19. These have very few peaks in the middle of the gradient where most peptides would cluster and slightly more peaks corresponding to hydrophobic molecules, more than likely lipids. The change in the chromatograms between the exosome derived proteins and the proteins derived from a whole cell lysate with a comparatively high protein abundance is almost directly related to the protein concentration of the sample loaded on the MS. The exosome data show low protein abundances, toward the lower end of detection in the DC Protein Assay. It is used to demonstrate the relationship between loaded protein and the resulting chromatograms. Although visually assessing a chromatogram is not a reliable way of predicting the number of protein IDs generated in Proteome Discoverer, it is generally possible to determine the characteristic chromatograms of a low protein abundance sample (Figure 4-18, Figure 4-19). Note that the lysis method used in some samples of this section is a variation of the $8 \mathrm{M}$ urea lysis solution containing $6 \mathrm{M}$ urea and $2 \mathrm{M}$ thiourea. This method was discontinued as the thiourea would crystallize at low temperatures which was inconvenient for later centrifugation steps.

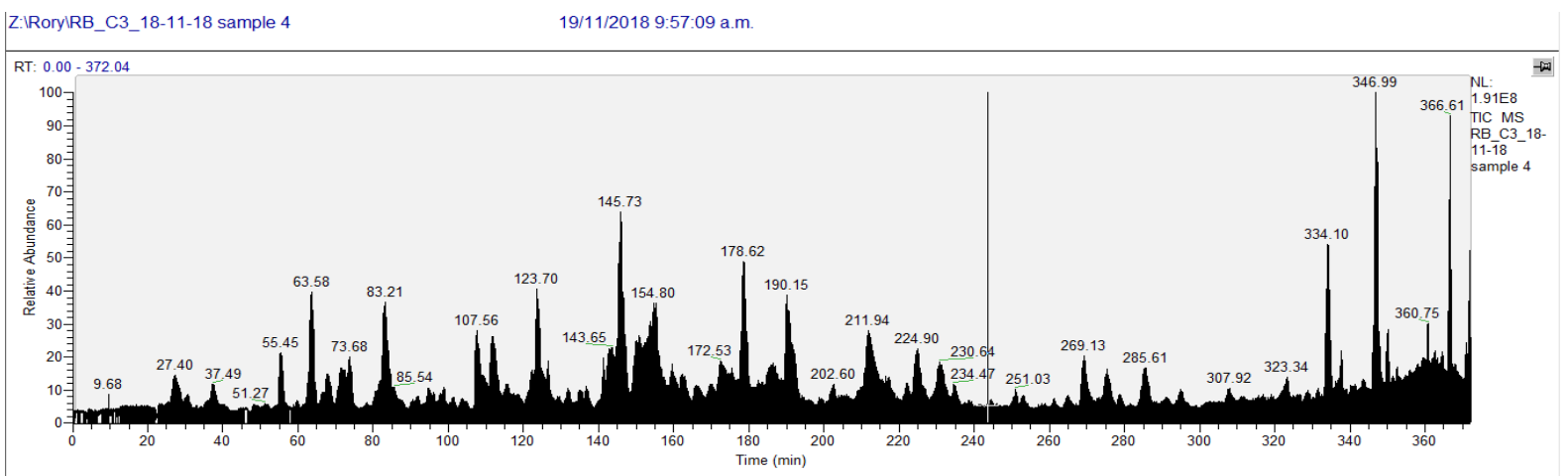

Figure 4-18 LC-MS/MS total ion count chromatogram of exosomes lysed using 6M urea $+2 \mathrm{M}$ thiourea. Number of recognised proteins: <50. The sample was obtained using $8 \mathrm{M}$ urea lysis to replace the RIPA lysis buffer, exosomes prepared by Deanna Dupre. included to show the resulting spectra when peptide concentration is insufficient.

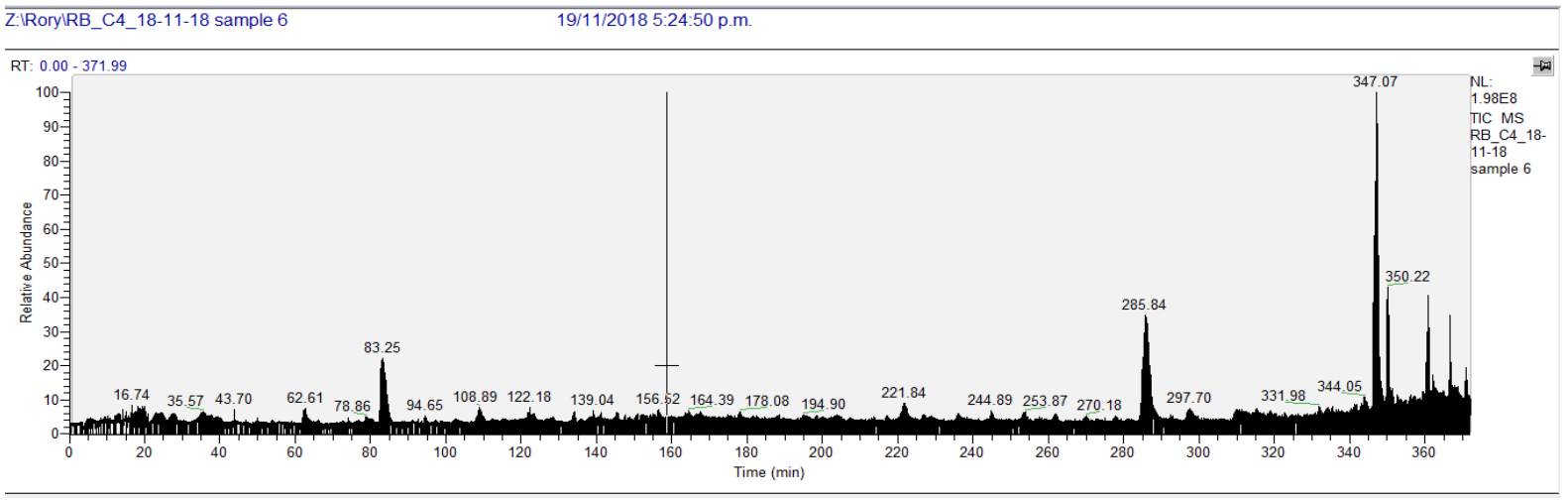

Figure 4-19 LC-MS/MS total ion count chromatogram of exosomes lysed using 6M urea $+2 \mathrm{M}$ thiourea. Number of recognised proteins: <10. The protocol I used to replace the RIPA lysis buffer, exosomes prepared by Deanna Dupre included to show the resulting spectra when peptide concentration is insufficient. 


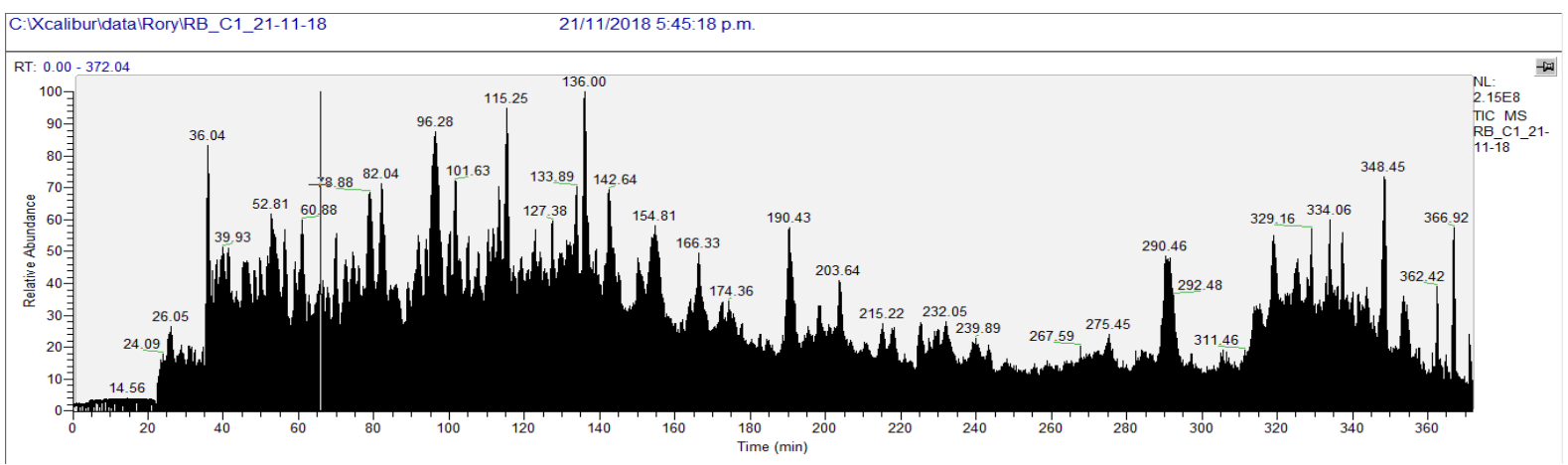

Figure 4-21 LC-MS/MS total ion count chromatogram of HT29 cells lysed using 6M urea $+2 M$ thiourea. Number of proteins identified with a high level of confidence in proteome discoverer: 967

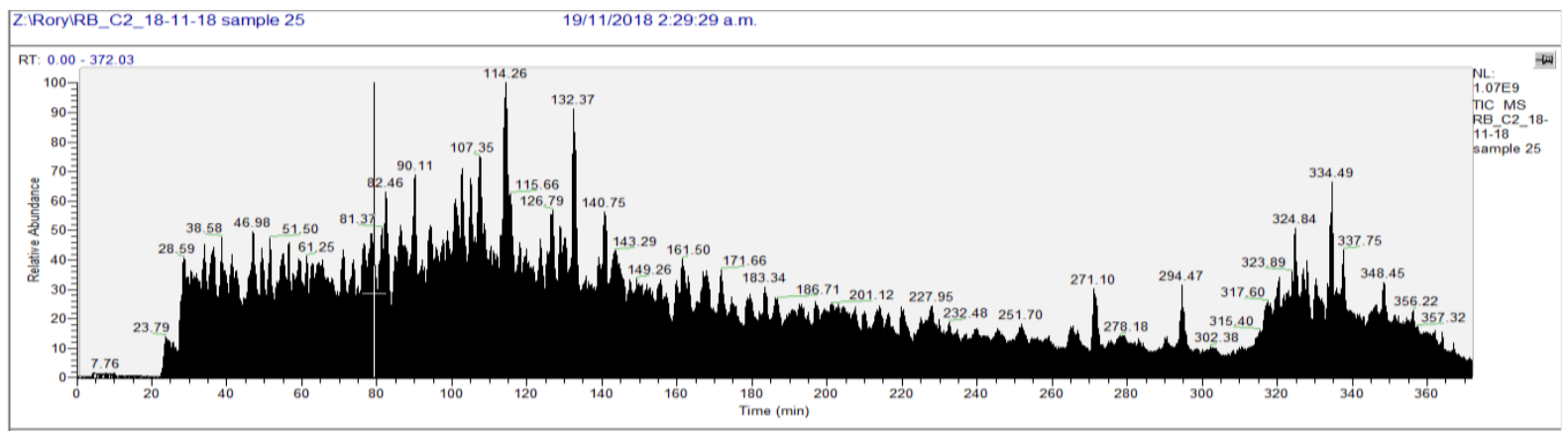

Figure 4-20 LC-MS/MS total ion count chromatogram of HT29 cells lysed using 6M urea $+2 M$ thiourea. Number of proteins identified with a high level of confidence in proteome discoverer: 1234 . Demonstrating the effectiveness of my digestion and processing protocol.

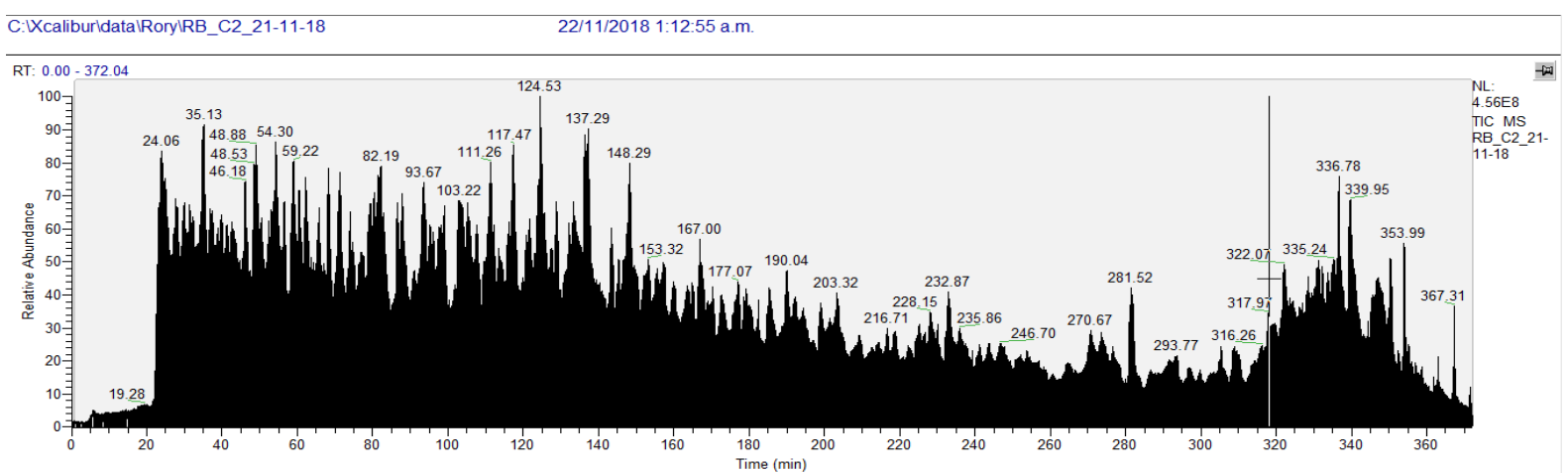

Figure 4-22 LC-MS/MS total ion count chromatogram of HT29 cells lysed using 6M urea $+2 M$ thiourea. Number of proteins identified with a high level of confidence in proteome discoverer: 1286 


\subsection{Mass spectrometry results}

It will become apparent in the MS results that data was only collected in the HT29 cell line. This was due in part to the difficulties encountered with culturing the HL60 cells and in part to availability of the mass spectrometer as it is a shared instrument with high usage and was unavailable for large periods of time whilst under repair. A mirror experiment was set up in the HL60 cell line and the cells processed to the point where they could be analysed on the mass spectrometer, however, in the interest of time and availability of the instrument, the HT29 cell data was the focus of the MS analyses.First Set of MS results: HT29 cells - 4E1RCat $(3.2 \mu \mathrm{M})$ and DMSO at $(0.4 \% \mathrm{v} / \mathrm{v})$ This set of data was not used to evaluate the effects of 4E1Rcat. It is provided as example of the effects of the protein loading concentration (total protein) on the number of protein hits in Proteome Discoverer. There is a correlation between total protein loaded and the number of proteins identified. In this preliminary experiment, a calculation error meant that the appropriate amount of 4E1RCat was not used, the target concentration of $40 \mu \mathrm{M}$ was missed and cells were instead treated at $3.2 \mu \mathrm{M}$. The matching DMSO control data was obtained at the $\mathrm{IC}_{10}$ of DMSO, rather than at the DMSO concentration that corresponded to the 4E1RCat treatment. 8M Urea (instead of $6 \mathrm{M}$ urea $+2 \mathrm{M}$ thiourea) was used as the lysis buffer. Membranes were not pelleted after cell lysis, protein precipitation occurred in acetone with membranes still present, protein concentrations post-precipitation were highly variable.

Table 3: More proteins are identified in Proteome Discoverer in response to higher total protein run on the MS. Displayed are: Samples 1-3: DMSO (0.4\% v/v), 4-6: 4E1RCat (3.2 $\mu \mathrm{M})$

\begin{tabular}{|c|c|c|c|c|}
\hline Sample \# & $\begin{array}{l}A_{750} \\
\text { (averaged } \\
\text { triplicate) }\end{array}$ & $\begin{array}{l}\text { Protein conc. } \\
(\mu \mathrm{g} / \mu \mathrm{L})\end{array}$ & $\begin{array}{l}\text { Total protein } \\
(\mu \mathrm{g})\end{array}$ & $\begin{array}{l}\text { Protein IDs } \\
\text { (Replicate } 1 ; 2 \text { ) }\end{array}$ \\
\hline Control - 1 & 0.224 & 0.8 & 26.5 & $834 ; 783$ \\
\hline Control - 2 & 0.267 & 1.2 & 41.4 & $781 ; 786$ \\
\hline Control - 3 & 0.231 & 0.8 & 28.5 & $926 ; 849$ \\
\hline 4E1RCat - 4 & 0.354 & 2.5 & 87.3 & $1211 ; 1182$ \\
\hline 4E1RCat - 5 & 0.293 & 1.5 & 52.8 & $1040 ; 1039$ \\
\hline 4E1RCat - 6 & 0.271 & 1.2 & 43.1 & 985; 959 \\
\hline
\end{tabular}

Samples were reconstituted in a final volume of $110 \mu \mathrm{L}$ of $0.1 \%$ formic acid. The protein yield was sampled post acetone precipitation, and large amounts of variability between samples was observed. The correlation between protein yield and the number of proteins identified in Proteome Discover influenced the methods used for later experiments. Samples for subsequent experiments that had low initial concentrations were normalised by reconstituting the sample in a lower volume, thus increasing the concentration of protein. The mass spectrometer uses $25 \mu \mathrm{L}$ for each replicate, and two technical replicates was considered enough. Therefore between 60 and $110 \mu \mathrm{L}$, there is room to adjust sample volume to increase the consistency of protein identification.

\subsubsection{Second set of MS results: HT29 cells - Untreated, CHX (0.043 $\mu \mathrm{M})$ and RBV $(11.1 \mu \mathrm{M})$}


Table 4: Normalising protein abundance to $100 \mu \mathrm{g}$ of total protein run on the MS leads to a large improvement in the number of proteins identified in Proteome Discoverer. Displayed: 1-3: Untreated cells, 3-6: Cycloheximide (0.043 $\mu$ M), 7-9: Ribavirin $(11.1 \mu \mathrm{M})$

\begin{tabular}{|c|c|c|c|c|c|c|}
\hline $\begin{array}{l}\text { HT29 } \\
\text { Sample \# }\end{array}$ & $\begin{array}{l}A_{750} \\
\text { (averaged } \\
\text { triplicate) }\end{array}$ & $\begin{array}{l}\text { Protein } \\
\text { Conc. } \\
(\mu \mathrm{g} / \mu \mathrm{L})\end{array}$ & $\begin{array}{l}\text { Total } \\
\text { protein } \\
(\mu \mathrm{g})\end{array}$ & $\begin{array}{l}\text { Dilution } \\
\text { factor }\end{array}$ & $\begin{array}{l}\text { Normalised } \\
\text { protein } \\
\text { abundance } \\
(\mu \mathrm{g})\end{array}$ & $\begin{array}{l}\text { Protein IDs } \\
\text { (Replicate } 1 ; 2 \text { ) }\end{array}$ \\
\hline Control - 1 & 0.484 & 3.9 & 290.6 & 2.9 & 100 & $1209 ; 1137$ \\
\hline Control - 2 & 0.457 & 3.3 & 250.7 & 2.5 & 100 & $1171 ; 1169$ \\
\hline Control - 3 & 0.392 & 2.2 & 168.1 & 1.7 & 100 & $1362 ; 1246$ \\
\hline $\mathrm{CHX}-4$ & 0.460 & 3.4 & 255.0 & 2.6 & 100 & $1315 ; 1209$ \\
\hline $\mathrm{CHX}-5$ & 0.476 & 3.7 & 278.5 & 2.8 & 100 & $1010 ; 1100$ \\
\hline CHX - 6 & 0.311 & 1.2 & 93.5 & $\mathrm{n} / \mathrm{a}$ & 93.5 & $1291 ; 1226$ \\
\hline RBV - 7 & 0.402 & 2.4 & 179.5 & 1.8 & 100 & $1106 ; 1096$ \\
\hline RBV - 8 & 0.376 & 2.0 & 151.0 & 1.5 & 100 & $1264 ; 1121$ \\
\hline RBV - 9 & 0.296 & 1.1 & 82.7 & $\mathrm{n} / \mathrm{a}$ & 82.7 & $1291 ; 1149$ \\
\hline
\end{tabular}

4.7.2 Third set of results: HT29 cells - DMSO control (1\%) and 4E1RCat (40 $\mu \mathrm{M})$

This data was used for subsequent analysis, and replaced data obtained from the first set of MS results.

Table 5: Normalising protein abundance leads to a consistent improvement in the number of identified proteins. Displayed are: Samples 1-3: DMSO (1\% v/v), 3-6: 4E1RCat (40 $\mu \mathrm{M})$

\begin{tabular}{|c|c|c|c|c|c|c|}
\hline $\begin{array}{l}\text { HT29 } \\
\text { Sample \# }\end{array}$ & $\begin{array}{l}A_{750} \\
\text { (averaged } \\
\text { triplicate) }\end{array}$ & $\begin{array}{l}\text { Protein } \\
\text { Conc. } \\
(\mu \mathrm{g} / \mu \mathrm{L})\end{array}$ & $\begin{array}{l}\text { Total } \\
\text { protein } \\
(\mu \mathrm{g})\end{array}$ & $\begin{array}{l}\text { Dilution } \\
\text { factor }\end{array}$ & $\begin{array}{l}\text { Normalised } \\
\text { protein } \\
\text { abundance } \\
(\mu \mathrm{g})\end{array}$ & $\begin{array}{l}\text { Protein IDs } \\
\text { (Replicate 1;2) }\end{array}$ \\
\hline Control - 1 & 0.309 & 1.6 & 123.0 & 1.2 & 100 & $1120 ; 1172$ \\
\hline Control - 2 & 0.354 & 2.2 & 167.3 & 1.7 & 100 & $1389 ; 1242$ \\
\hline Control - 3 & 0.333 & 1.9 & 145.6 & 1.5 & 100 & $1218 ; n / a$ \\
\hline 4E1RCat - 4 & 0.357 & 2.3 & 170.6 & 1.7 & 100 & $1200 ; \mathrm{n} / \mathrm{a}$ \\
\hline 4E1RCat - 5 & 0.344 & 2.1 & 156.8 & 1.6 & 100 & $1040 ; 1395$ \\
\hline 4E1RCat - 6 & 0.301 & 1.5 & 116.0 & 1.2 & 100 & $1235 ; 1122$ \\
\hline
\end{tabular}

\subsection{Further analysis of MS data post-protein identification}

After processing in Proteome Discoverer, data was moved into Scaffold and four treatment conditions were constructed and analysed. Comparisons were as follows: untreated controls against the cycloheximide (CHX) treatment, untreated controls against ribavirin (RBV), a 1\% DMSO control against 4E1RCat and lastly untreated controls versus the $1 \%$ DMSO control, essentially a DMSO treatment condition. 


\subsubsection{Gene ontological enrichment analysis using $\mathbf{G}$ profiler}

Global proteome changes were computed in Scaffold prior to GO analysis in the $\mathrm{G}$ profiler online software. Inclusion as a significant change was managed internally by the Scaffold software, which uses a decoy generation strategy to manage the FDR. No formal cut-off was applied with respect to protein abundance in terms of fold-changes. Despite this, on closer inspection the Scaffold software appears to have imposed cut-off boundaries, the lower threshold for an upregulated protein based on the four analyses run in Scaffold is a fold-change of $>1.1$ and the lower threshold for a downregulated protein appeared to be capped at a fold change of $>0.0002$.

Table 6 gene ontology enrichment by biological process (BP) and cellular component (CC). The molecular function (MF) GO domain was included in one analysis. The top 5 BP and CC are displayed, the intention of the selection process was to pick the top 5 unique processes, components and MF if applicable. If 5 unique processes did not exist, the nested ontology terms were included, despite the smaller ontology contributing to the enrichment seen in the larger ontology. Ontological enrichments are reported separately for proteins that increase in abundance (UPs) in response to the treatment, and those that go down (DOWNs). Ontology term name, adjusted p-values from G:profiler and the number of proteins observed to change in abundance that are assoicated with the ontology (intersection size) are reported.

\begin{tabular}{|l|l|r|r|}
\hline Cycloheximide UPs & & & \\
\hline source & term name & $\begin{array}{l}\text { adjusted } p \\
\text { value }\end{array}$ & $\begin{array}{l}\text { intersection } \\
\text { size }\end{array}$ \\
\hline GO:BP & RNA splicing, via transesterification reactions with bulged adenosine as nucleophile & $4.57 \times 10^{-2}$ & $4.57 \times 10^{-2}$ \\
\hline GO:BP & mRNA splicing, via spliceosome & 6 & 6 \\
\hline GO:BP & RNA splicing, via transesterification reactions & $6.80 \times 10^{-2}$ & $1.53 \times 10^{-5}$ \\
\hline GO:CC & ribonucleoprotein complex & $6.10 \times 10^{-5}$ & 11 \\
\hline GO:CC & catalytic step 2 spliceosome & $8.82 \times 10^{-5}$ & \\
\hline GO:CC & spliceosomal complex & 6 \\
\hline
\end{tabular}

\begin{tabular}{|c|c|c|c|}
\hline $\begin{array}{l}\text { Cycloheximide } \\
\text { DOWNs }\end{array}$ & & & \\
\hline source & term name & $\begin{array}{l}\text { adjusted } \mathrm{p} \\
\text { value }\end{array}$ & $\begin{array}{l}\text { intersection } \\
\text { size }\end{array}$ \\
\hline GO:BP & regulation of protein catabolic process & $1.07 \times 10^{-4}$ & 9 \\
\hline GO:BP & positive regulation of protein modification by small protein conjugation or removal & $4.63 \times 10^{-4}$ & 6 \\
\hline GO:BP & regulation of protein modification by small protein conjugation or removal & $6.19 \times 10^{-4}$ & 7 \\
\hline GO:BP & proteolysis involved in cellular protein catabolic process & $2.20 \times 10^{-3}$ & 10 \\
\hline GO:BP & proteasome-mediated ubiquitin-dependent protein catabolic process & $2.80 \times 10^{-2}$ & 7 \\
\hline
\end{tabular}




\begin{tabular}{|l|} 
GO:CC \\
\hline GO:CC \\
\hline GO:CC \\
\hline GO:CC \\
\hline GO:CC \\
\hline
\end{tabular}

proteasome complex

$2.10 \times 10^{-3}$ endopeptidase complex

$2.23 \times 10^{-3}$

peptidase complex

ubiquitin conjugating enzyme complex

$6.80 \times 10^{-3}$

$3.16 \times 10^{-2}$

MCM complex

$4.81 \times 10^{-2}$

2

\begin{tabular}{|c|c|c|c|}
\hline \multicolumn{4}{|c|}{ Ribavirin UPs } \\
\hline source & term name & $\begin{array}{l}\text { adjusted } p \\
\text { value }\end{array}$ & $\begin{array}{l}\text { intersection } \\
\text { size }\end{array}$ \\
\hline $\mathrm{GO}: \mathrm{BP}$ & nuclear-transcribed mRNA catabolic process, nonsense-mediated decay & $1.41 \times 10^{-4}$ & 7 \\
\hline GO:BP & SRP-dependent cotranslational protein targeting to membrane & $7.72 \times 10^{-4}$ & 6 \\
\hline GO:BP & RNA catabolic process & $1.46 \times 10^{-3}$ & 9 \\
\hline GO:BP & ribosome biogenesis & $3.79 \times 10^{-2}$ & 7 \\
\hline GO:BP & maturation of LSU-rRNA & $3.14 \times 10^{-2}$ & 3 \\
\hline GO:CC & ribonucleoprotein complex & $2.81 \times 10^{-8}$ & 17 \\
\hline GO:CC & cytosolic ribosome & $2.73 \times 10^{-4}$ & 6 \\
\hline GO:CC & nucleolus & $3.71 \times 10^{-4}$ & 13 \\
\hline GO:CC & polysome & $5.82 \times 10^{-4}$ & 5 \\
\hline GO:CC & cytosolic part & $1.42 \times 10^{-3}$ & 7 \\
\hline
\end{tabular}

\begin{tabular}{|c|c|c|c|}
\hline Ribaviri & & & \\
\hline source & term name & $\begin{array}{l}\text { adjusted } p \\
\text { value }\end{array}$ & $\begin{array}{l}\text { intersection } \\
\text { size }\end{array}$ \\
\hline GO:MF & cadherin binding involved in cell-cell adhesion & $1.34 \times 10^{-3}$ & ( \\
\hline GO:MF & cadherin binding & $4.30 \times 10^{-3}$ & 6 \\
\hline GO:MF & cell-cell adhesion mediator activity & $2.62 \times 10^{-2}$ & 3 \\
\hline GO:MF & cell adhesion mediator activity & $4.30 \times 10^{-2}$ & 3 \\
\hline GO:MF & cell adhesion molecule binding & $4.35 \times 10^{-2}$ & 6 \\
\hline GO:CC & cell-cell adherens junction & $5.65 \times 10^{-3}$ & 4 \\
\hline
\end{tabular}




\begin{tabular}{|l|}
\hline GO:CC \\
\hline GO:CC \\
\hline GO:CC \\
\hline GO:CC \\
\hline
\end{tabular}

adherens junction proteasome complex

$3.30 \times 10^{-2}$

endopeptidase complex

anchoring junction

$3.45 \times 10^{-2}$

$3.74 \times 10^{-2}$

\begin{tabular}{|c|c|c|c|}
\hline 4E1RCat UPs & & & \\
\hline source & term name & $\begin{array}{l}\text { adjusted } p \\
\text { value }\end{array}$ & $\begin{array}{l}\text { intersection } \\
\text { size }\end{array}$ \\
\hline $\mathrm{GO}: \mathrm{BP}$ & SRP-dependent cotranslational protein targeting to membrane & $2.46 \times 10^{-7}$ & 7 \\
\hline GO:BP & nuclear-transcribed mRNA catabolic process, nonsense-mediated decay & $1.34 \times 10^{-6}$ & 7 \\
\hline GO:BP & translational initiation & $1.13 \times 10^{-6}$ & 8 \\
\hline GO:BP & heterocycle catabolic process & $3.03 \times 10^{-5}$ & 10 \\
\hline GO:BP & ribosomal large subunit biogenesis & $9.21 \times 10^{-3}$ & 4 \\
\hline GO:CC & cytosolic large ribosomal subunit & $1.26 \times 10^{-9}$ & 7 \\
\hline GO:CC & large ribosomal subunit & $2.16 \times 10^{-9}$ & 8 \\
\hline GO:CC & ribonucleoprotein complex & $4.62 \times 10^{-7}$ & 12 \\
\hline GO:CC & cytosolic part & $1.57 \times 10^{-5}$ & 7 \\
\hline GO:CC & polysomal ribosome & $2.72 \times 10^{-5}$ & 4 \\
\hline
\end{tabular}

\begin{tabular}{|c|c|c|c|}
\hline 4E1RCa & & & \\
\hline source & term name & $\begin{array}{l}\text { adjusted } p \\
\text { value }\end{array}$ & $\begin{array}{l}\text { intersection } \\
\text { size }\end{array}$ \\
\hline GO:CC & nucleoid & $1.48 \times 10^{-3}$ & 3 \\
\hline GO:CC & mitochondrial nucleoid & $1.48 \times 10^{-3}$ & 3 \\
\hline GO:CC & nuclear replisome & $4.27 \times 10^{-2}$ & 2 \\
\hline
\end{tabular}




\begin{tabular}{|c|c|c|c|}
\hline $\begin{array}{l}\text { DMSO UPs } \\
\text { source }\end{array}$ & term name & $\begin{array}{l}\text { adjusted } p \\
\text { value }\end{array}$ & $\begin{array}{l}\text { intersection } \\
\text { size }\end{array}$ \\
\hline $\mathrm{GO}: \mathrm{BP}$ & SRP-dependent cotranslational protein targeting to membrane & $3.20 \times 10^{-25}$ & 24 \\
\hline GO:BP & translational initiation & $7.82 \times 10^{-20}$ & 26 \\
\hline GO:BP & nuclear-transcribed mRNA catabolic process, nonsens $x$ 10-mediated decay & $1.71 \times 10^{-22}$ & 24 \\
\hline GO:BP & nuclear-transcribed mRNA catabolic process & $1.19 \times 10^{-16}$ & 24 \\
\hline GO:BP & ribosome biogenesis & $9.87 \times 10^{-6}$ & 16 \\
\hline GO:CC & cytosolic ribosome & $1.21 \times 10^{-25}$ & 25 \\
\hline GO:CC & cytosolic part & $8.23 \times 10^{-19}$ & 26 \\
\hline GO:CC & mitochondrial matrix & $4.27 \times 10^{-10}$ & 24 \\
\hline GO:CC & mitochondrial nucleoid & $2.48 \times 10^{-8}$ & 9 \\
\hline GO:CC & cell-substrate adherens junction & $5.69 \times 10^{-7}$ & 19 \\
\hline
\end{tabular}

\begin{tabular}{|c|c|c|c|}
\hline \multicolumn{4}{|c|}{ DMSO DOWNs } \\
\hline source & term name & $\begin{array}{l}\text { adjusted } p \\
\text { value }\end{array}$ & $\begin{array}{l}\text { intersection } \\
\text { size }\end{array}$ \\
\hline $\mathrm{GO}: \mathrm{BP}$ & RNA splicing, via transesterification reactions & $3.61 \times 10^{-7}$ & 21 \\
\hline GO:BP & protein folding & $4.19 \times 10^{-7}$ & 17 \\
\hline GO:BP & translation & $4.68 \times 10^{-7}$ & 30 \\
\hline GO:BP & negative regulation of mRNA metabolic process & $5.71 \times 10^{-7}$ & 11 \\
\hline GO:BP & regulated exocytosis & $2.10 \times 10^{-6}$ & 30 \\
\hline GO:CC & secretory granule lumen & $1.58 \times 10^{-11}$ & 24 \\
\hline GO:CC & focal adhesion & $1.09 \times 10^{-7}$ & 22 \\
\hline GO:CC & chaperone complex & $7.91 \times 10^{-6}$ & 6 \\
\hline GO:CC & proteasome complex & $1.23 \times 10^{-5}$ & 9 \\
\hline GO:CC & actin cytoskeleton & $6.81 \times 10^{-5}$ & 20 \\
\hline
\end{tabular}

The full tables generated from the GO analysis are provided in Appendix Table 11, Table 12, Table 13, Table 14. They are large and contain nested ontological terms, in which an

ontological hierarchy is enriched by the ontologies nested below it. This table was constructed as a way to highlight the processes and cellular compartments in which our treatments seem to be generating the most significant enrichments in the HT29 cell line, revealing the major cellular response to the treatments. 


\section{Discussion}

\subsubsection{The research questions}

The research performed in this thesis aims to gather more information surrounding the unknown consequences of inhibiting the elF4E component of the elF4F complex. Pateamine A has demonstrated the ability to selectively disturb protein expression at low doses, such that there is not a global lowering of translation in proportion to the disturbance, but rather has selective effects with interesting outcomes. Other elF4A inhibitors, hippuristanol and silvestrol have mirrored these effects in vitro and the outcome of this selective lowering of protein expression has demonstrable therapeutics effects in vivo, namely the rescue of cachexia. Research has demonstrated that rocaglates can change the sequence selectivity of the 4A helicase with its mRNA target (Iwasaki, Floor, \& Ingolia, 2016) - this selectivity is facilitated by motifs in the mRNA 5' UTR, and is one potential explanation for the non-uniform effects of Pateamine $A$ on the proteome.

Previous research by our laboratory has demonstrated that at low dose treatment, equivalent to an $\mathrm{IC}_{10}$ or below in the cell lines treated, Pateamine $A$ disturbs protein expression in a pattern across the global proteome. This in contrast to the selected control drug, cycloheximide which uniformly lowers global protein expression. This research was an extension of the proteomic work with Pateamine A. Treatment involved two main drugs, 4E1RCat and ribavirin, 4E1RCat acts at the elF4EelF4G interface preventing interaction and so interfering with cap-recognition. Ribavirin acts as an m7G cap mimic and competes with mRNA vying for translation. This distinction may have functional outcomes as eIF4E interaction with eIF4G is likely to have a regulatory or stimulatory effect.

In this research, the elF4F complex was perturbed by 4E1RCat and ribavirin treatment and the proteomic outcome investigated to see if a consistent pattern emerged. This research attempted to generate insight into inhibition of elF4F in the context of cachexia. It is still uncertain how the cachexia rescuing effects of elF4A inhibitors are mediated. The work was therefore designed to address a hypothesis that the selective anti-cachectic effects of inhibition of elF4A are mediated by the elF4F complex.

The structure of the experiments I undertook was chosen to attempt to answer a core set of questions:

1. Is there a pattern in the upregulation or downregulation of proteins in response to low dose elF4E inhibition? Does this pattern mirror the effects seen with elF4A inhibition?

2 Are there elF4E dependent transcripts and what characteristics (e.g. motifs in the $5^{\prime}$ UTR), if any, do they possess? Do these characteristics mirror those seen with elF4A inhibition?

In answering these questions, I hoped to identify whether the anti-cachectic effects of inhibition of elF4A is solely mediated by elF4A itself, or whether there is a potential effector role for the elF4F complex as a whole. Furthermore, the work should reveal whether there is a possibility of therapeutic intervention at elF4E to ameliorate cachexia, much like elF4A inhibition. 


\subsection{Practical work and technique focused discussion}

\subsubsection{Cell culture}

\subsubsection{Obtaining reproducible treatment data}

Getting reproducible drug response data from multiple MTT assays was a challenge. The low solubility of 4E1Rcat in comparison to ribavirin and cycloheximide caused significant trouble when collecting treatment data. Low solubility combined with 4E1RCat's low toxicity and the cytotoxic effects of DMSO was a major challenge for data collection and made the task of fitting a non-linear regression open to interpretation. The variability of the data could be caused by several factors, one of them I addressed by the running an assay at two cell concentrations. Fig 6.6 shows the effects of cell number on the outcome of an MTT assay. I noticed early on that using the standard number of cells $(10,000)$ appropriate for an HL-60 assay made the wells look exceptionally crowded when using the significantly larger HT-29 cells. In response to this, I ran an assay to check how cell number would affect the outcome of an assay. Decreasing the number of cells to 2,500 has a subtle, but noticeable effect at the lower drug concentrations, with a steeper drop in cell activity in comparison to the other three curves. Although the other three curves show significant variability at the high drug concentrations, they show a marked consistency at the lower doses.

In the MTT assay results of 4E1RCat in the HT29 cell line, there appeared to be limited absorbance changes, even at high concentration. This may be due low efficacy of the compound in this cell line. However, I wished to establish that it did not arise from the optical properties of the 4E1RCat treatment, as the compound has both a strong optical absorbance and low aqueous solubility, potentially leading to scattering effects. However, formazan crystals were formed during the MTT metabolism step of the assay in the treated $\mathrm{HT} 29$ cells, confirming that they remain metabolically active. Furthermore, the equivalent assay with HL60 cells does show a complete loss of metabolic activity showing that the 4E1RCat solution is unlikely to interfere with the absorbance readings from the MTT assay. This trend of cell quiescence was seen consistently across all the HT29 MTT assays.

\subsubsection{Practicalities of cell maintenance}

\subsection{Growth characteristics relate to freezing methodology}

The original stocks from which cells in this thesis were derived grew exceptionally well, despite being nearly 20 years old (frozen in 1998). HT29 cells grown over the course of this thesis were aggressively detached using a combination of trypsin and EDTA. When detaching cells from culture flasks, initially the goal was to separate the cells into individual 'colony forming units', however, cells thawed using this method tended to grow at a significantly slower rate than observed in the original stocks. Original liquid nitrogen stocks recovered from the thawing process with notable ease and rapidly adhered and begun to grow within 1-2 days. Closer inspection of the original stocks late in this thesis yielded some insights: cells from the original stocks were cryopreserved in clusters, which meant that cells must have been less aggressively detached from their cell culture flasks. These cells could have been frozen using a different method such as the 'Mr. Frosty' cryogenic isopropanol containers, the concentration of cryoprotectants or freezing media used may also have been optimised. Freezing cells in clusters is likely to be the main reason for the original stocks' excellent post-thaw growth.

\subsection{HL60 cells}

The HL60 suspension cells used in this thesis generally behaved well. However, growing cultures growing would frequently collapse, seemingly without explanation. It is unlikely that this is due to cells becoming over-confluent, as suggested by empirical evidence in the form experiments with 
taking cells left in the incubator with the same media for $>2$ weeks and coaxing the cells back to a healthy state with fresh media. This was done out of an interest in understanding the cell line better. It seems to indicate that HL60 cells are robust to the effects of metabolic acids and high confluency. Another separate issue with HL60 cells that added to trouble in the tissue cell culture suite seemed to arise from cells that were frozen poorly or thawed poorly, leading to large numbers of dead cells. Unlike adherent cells, removing debris is a challenge when dealing with suspension cell lines. Attempts to slow-centrifuge cells in the hope that the cells which should have more mass than the debris surrounding them would be enriched showed little benefit. Even at the lowest speed settings, the centrifuged pellet would contain still contain significant quantities of debris.

\subsection{HT29 cells}

HT29 cells have peculiar growth patterns, distinct from most other cell lines observed over the course of this thesis. They tend to form pseudo-glandular structures and grow in an almost bacterial manner, with the colony expanding and even growing in a 3-D orientation rather than the traditional adherent cells which tend to form a monolayer, see Figure 6-2. The nature of HT29 cell growth made visual assessments of confluence challenging, a combination of observing the coverage of the flask's surface and the colour of phenol red in culture media was required to assess proximity to the confluence threshold. HT29 cells, much like the HL60 cells, suffered from the same issues seen where the culture seemed to collapse for no apparent reason. Similar experiments were done with HT29 cells regarding response to metabolic acid and high confluency, and the conclusion was again that HT29 cell culture collapse is unlikely to be due to these factors, refer to Figure 6-3, Figure 6-4, Figure 6-5.

\subsection{Rationale for removing PenStrep from media}

Cell culture was initially performed using a pre-made mix of penicillin and streptomycin. Use of antibiotics in cell culture media was discontinued prior to data collection using MTT assays and drug treatment regimens for several reasons. PenStrep was avoided primarily due to research demonstrating genome wide gene regulation and expression changes caused by anti-biotic use in cell culture implicating a molecular interaction within cells (Ryu, Eckalbar, Kreimer, Yosef, \& Ahituv, 2017). Secondarily, bacteria can grow in media laden with antibiotics, with a sufficiently large bacterial or fungal load in a contamination event. Cell culture could be accurately described as a waiting game. Cultures where PenStrep is present will take longer for a contamination to become evident; removing PenStrep allowed mistakes to be rectified and new cultures to be started, generally within 24 hours of a contamination event.

\subsection{Problems with thawing, absence of glutamine and contamination}

Over the course of this thesis extensive trouble was encountered when culturing cells. The cause being three-fold. When attempting to start up cultures from freezer stocks, the recovery of cells from thaw, an unnoticed change to L-glutamine-free media, and periodic contamination compounded to make identifying the sources of problems difficult. Effectively caring for cells that have undergone sub-optimal freezing or thawing was a skill learned late in this thesis. Rinsing off dead cells was especially useful for adherent cells, as dead cells and debris can be selectively removed from the culture. Dead cells can acidify media inducing metabolic stress, and debris and secreted molecules from dying cells were also observed to inhibit the sustained, healthy growth of cells in culture. Initial orders of RPMI-1640 contained glutamine, the indicator of the presence or lack of glutamine is a small piece of text on the bottle. At various points during the year cells were dying for seemingly no reason, it is suspected that various orders of media with or without glutamine may explain this. The most useful approach was getting an experienced cell culture user to observe and criticise my technique in the cell culture environment, it became apparent that 
assumptions about the sterility of pieces of equipment that had not been autoclaved had been made, this is likely to be a significant source of contamination across the course of this thesis. Trouble-shooting in the cell culture environment was an important component of this thesis, identifying the problem is the main issue and several simple tests were employed to quickly determine what was causing the problem. For contamination issues, the first step was to test potentially contaminated reagents by transferring an appropriate amount into uncontaminated media and incubating it for 1-2 days, to see if any bacteria or fungi grew. For cells that were not growing well, a different stock of the same cell line could be thawed to see if the problem was isolated at the individual level to the liquid nitrogen stock. It could also be checked if media was causing growth issues by seeing if an available HeLa cell line would grow in the media causing trouble. Alternatively, cells could be given more time to recuperate, and be given new media every few days to see if they could make a recovery.

It was noted that HT29 cells grow slowly in media without glutamine, but HL60 cells start to die and refuse to grow, this may have been a fourth confounding factor that made identifying the problem more difficult, this was realised late in the thesis.

\subsection{A criticism of cell counting}

Cell counting was an important component of this project. However, it was also something that seemed to be a ballpark measure at times. The variability is likely to be a result of three phenomena, firstly, although the samples were thoroughly mixed by pipette prior to sampling, after a sample had been removed and placed on the glass adjacent to the hemocytometer squares, cells would have a chance to settle to the bottom within the globule of media. The second source of variability was the inconsistent pattern of capillary-like uptake of media between the glass coverslip and the glass of the hemocytometer. This inconsistency of can lead to some regions of the hemocytometer having significantly more or less cells. Lastly, small volumes were used, typically $2 \mu \mathrm{L}$ was diluted in $18 \mu \mathrm{L}$, a 10 -fold dilution, if the settling of cells in the globule of media is occurring then sampling using small volumes could encounter a problem when sampling of regions of lower or higher cell density. Prior to treatment with compounds, roughly 2 million HT29 or HL60 cells were plated. Whether 2 million cells were plated or 1.5 million cells or 2.5 million matters, see fig 6.6. There are also carry-over effects when it comes to sample processing on the mass spectrometer. Ideally there should be a comparable number of cells between treatment conditions and minimal normalisation towards the later stages, where the volume that peptides are reconstituted in can be varied to ensure consistent concentrations are run on the mass spectrometer. In this sense errors in cell counting could carry through all the way to mass spectrometry stage. There are two points in this project where an accurate cell count will have the most effect on experimental data, the cell counting required to plate 10,000 cells in an MTT well and the cell counting prior to plating up for drug treatment. Although we control for variability between MTT assays by taking absorbance as a percentage of a given control, accurately plating 10,000 cells per well every time improves the comparability of assays. The same principle applies to plating for drug treatment. Cell counting is time consuming and extends the hours spent in the tissue culture suite with every sample that requires counting. Increased consumable used in the form of plastic falcon tubes for the centrifugation process, and pipette tips for the counting are generally necessary, the extra handling can also occasionally result in contamination events, and the mental burden of doing mundane basic mathematics where mental energy could best be applied elsewhere. Cell counting using a hemocytometer is still an important skill in the wet-lab scientists' arsenal, however, it is 2019 and automated tools have existed for several years now, perhaps it is time to put away the archaic technology and move into the future. 


\subsubsection{Defining a biological replicate in the context of this study}

Biological replicates were defined as a culture that had spent at least one passage in its own flask before being seeded and used for either an MTT assay or a treatment at a selected concentration of drug. Technical replicates are defined as either two cultures seeded from the same initial cell culture and used as part of an MTT assay or drug treatment or, in the case of MS data collection, peptide solutions were sampled twice, and thus two technical replicates were combined for later analysis.

\subsubsection{Cell lysis and protein handling optimisation}

The preparatory steps prior to mass spectrometry involved cell lysis, protein precipitation, disulfide bridge removal, subsequent capping of cysteines with carbamidomethyl moieties, tryptic digestion, desalting and concentration of peptides. This process required extensive optimisation to improve the spectral data abundance and quality. The methodologies for cell lysis and protein precipitation were evaluated, and steps taken to increase peptide abundances as described below.

\subsubsection{Protein extraction/precipitation methodology choice}

Plastics used for protein-related work were not autoclaved to lower the risk of plasticiser leaching into samples. LoBind Tubes were also used as a precaution, reducing the risk of peptides bonding with the plastic when drying in the centrivap concentrator.

\subsection{Cell lysis/protein extraction optimisation}

Following recognition of PEG contamination, the lysis solution needed to be changed to one not containing non-ionic detergents as these are a source of PEG. The efficiency of several lysis solutions was assessed - RIPA buffer, $8 \mathrm{M}$ urea, $8 \mathrm{M}$ urea $+\mathrm{SDC}$, and $\mathrm{dH}_{2} \mathrm{O}$ with added protease inhibitor. The lysis solutions were also combined with 3 quick freeze/thaw cycles in a $-80^{\circ} \mathrm{C}$ freezer, with vortexing of lysate in between. This was an added step to increase the extraction efficiency. RIPA buffer is a purpose-designed lysis buffer and the most aggressive solution, liberating a total of $420 \mu \mathrm{g}$ of protein this is likely to be the maximum amount that can be extracted. Due to losses during the acetone precipitation a solution as close to the RIPA buffer efficiency as possible was desirable. Human cell lines are generally easy to lyse and don't require an aggressive lysis procedure for protein liberation; $8 \mathrm{M}$ urea is used as a solubilising agent in later steps in the protein extraction protocol, thus it was logical to test it as a lysis solution. The $8 \mathrm{M}$ urea lysis was simple and deemed efficient enough to be used extensively.

\subsection{Protein precipitation}

Two methods were investigated for protein precipitation - acetone, and chloroform-methanol (CM). I found the acetone precipitation to be simpler, more consistent, and predictable in its ability to precipitate proteins from cell lysates. Previous research performed by our laboratory using C-M suggested that it was the more effective of the two precipitation methods. The slightly more complex C-M precipitation outperformed the acetone precipitation in raw protein yield, but results were more variable overall. Ultimately, the reliability and utility of acetone precipitation were the deciding factors and this method was employed for the data acquisition phase of this project.

\subsection{Protein loading optimisation for LC-MS/MS}

A need for optimising protein loading concentration by normalising became apparent after the first round of mass spectrometry data was collected. This need came to light after the first round of data of 4E1RCat and DMSO treated HT29 cells was captured. A large discrepancy between protein abundance between samples 1- 6 was noted, with some as low as $\sim 25 \mu \mathrm{g}$ and some as high as $\sim 90$ $\mu \mathrm{g}$. This could have been a consequence of seeding density due to poor cell counting, loss of cells when aspirating media, or an error in the estimation of protein abundance using the DC assay. The 
analysis in graphpad was done using a non-linear regression to extract protein concentration using a standard curve of known protein abundances, this strategy may also have contributed to some of the variability. However, the incredibly strong correlation between post-precipitation protein concentration and the number of identified proteins in Proteome Discoverer suggest that the DC assay and non-linear regression are not at fault. The above observations are evidence that protein concentration in a sample and the number of protein IDs are strongly linked, indicating that using around $80-100 \mu \mathrm{g}$ is likely to give a high number of protein IDs.

\subsubsection{The polyethylene glycol saga}

\subsection{Rationale for exhaustive removal of PEG from samples}

The reason PEG contamination became an issue is two-fold. Another student doing affinity pulldown of a low abundance protein initially used RIPA buffer for cell lysis, the RIPA recipe includes Triton X-100 a non-ionic PEG containing detergent. This became an issue as PEG tends to lead to ion suppression of sample peptides. This becomes a significant issue when working with low abundance proteins where the sensitivity decrease caused by PEG ion suppression manifests as an inability to detect peptides from a sample. The second issue is that the mass spectrometer is a shared instrument and the PEG takes significant washing to remove from the LC column, another user interested in low-abundance proteins was concerned that the presence of PEG was lowering the sensitivity of the mass spectrometer, although later recognised as a contamination within their own samples. Previous research done by our lab group did not run into the problem of PEG contamination despite the use of RIPA buffer, this is likely because these experiments were observing a whole proteome and the protein abundances were well in excess of the PEG present. The work performed in this thesis is likewise a whole proteome approach with high protein abundances, and so should not theoretically have run into any issues, however, it was noted that a good run from the previous research was expected to yield $\sim 800$ protein IDs, and a bad run about $\sim 600$. In contrast, an excellent run over the course of this thesis would yield up to 1400 proteins and a bad run was expected to yield 800 or below protein IDs. This discrepancy of protein yields could come as direct consequence of ion suppression by PEG that may never have been addressed had such stringency in identifying and removing the source of PEG been implemented.

\subsection{MALDI-TOF use for PEG detection}

The MALDI-TOF was used as a tool for analysing samples because it is quick, simple and samples require less processing (desalting, tryptic digestion) before a sample can be analysed as MALDI is more tolerant to high salt concentrations. This allows PEG contamination to be detected more rapidly in comparison to the LC-MS/MS. For a given MALDI experiment the matrix, generally CHCA is combined with a few microlitres of sample at ratios of 1:10 and 1:100, a single microlitre is spotted and dried, this drying period can be very brief or, in the case of high urea concentrations can take up to an hour. Polyethylene glycol has a characteristic fragmentation pattern, with a difference of 44 mass units which closely resembles the shape of a normal distribution (Figure 4-11). In summary, MALDI-TOF is quick, simple, salt-tolerant and has low preparatory time, the 44-repeating pattern of PEG is easy to spot. Using this technique, samples were verified to be free of PEG to identify the ideal methodology for sample preparation for LC-MS/MS analysis. 


\subsubsection{Mass spectrometry}

\subsubsection{Mass spectrometry interpretation software}

Proteome Discoverer (Mascot, Sequest databases), Scaffold and the Princeton Edu FDR calculator were used to identify peptides and protein matches.

\subsection{Rationale of static and dynamic modification settings}

There are settings within Proteome Discoverer to address peptide modifications in a given proteomics experiment. A static modification refers to those modifications which have been intentionally imposed on the sample peptides, in this case cysteine residues were capped with carbamidomethyl groups from the iodoacetamide treatment to prevent disulfide bridges from reforming. Dynamic modifications refer to modifications that may arise non-uniformly to the sample imposed intentionally or unintentionally because of sample handling. Urea was used extensively as a lysis buffer and a means to re-dissolve proteins. At times the urea/sample solutions would reach as high as $56^{\circ} \mathrm{C}$, increasing the likelihood of carbamylation at the $\mathrm{N}$-terminus and at lysine residues. Oxidation at lysine residues, and deamidation of amide sidechains were also considered as dynamic modifications. Tryptic digestion occurs at lysine and arginine residues, and extensive carbamylation and oxidation can lead to poorly digested polypeptides, this did not become a problem during this thesis. Below is an example of protein ID numbers improving in response to addition of modifications. In addition, a readout has been added from the Proteome Discoverer interface showing a selection of heavily modified peptides some with multiple modifications per peptide chain. It is important to note that most peptides identified have no modifications, with a small proportion having 1 modification, usually carbamidomethylation at a cysteine residue, and an even smaller minority having 1 or more dynamic modification. Adjusting the dynamic modification settings resulted in a slight improvement to the number of proteins identified. Overall the processing methodologies used to prepare samples for LC-MS/MS analysis worked well, samples were not degrading and the effect of using urea was relatively minor.

\section{Only fixed carbamidomethylation (no dynamic)}

Total high-quality proteins: 1225

Total PSMs (peptide sequence matches): 22271

\section{2. +oxidation (only)}

Total high-quality proteins: 1234

Total PSMs (peptide sequence matches): 22655

\section{3. +carbamoylation (only)}

Total high-quality proteins: 1246

Total PSMs (peptide sequence matches): 22421

\section{4. +oxidation +carbamoylation}

Total high-quality proteins: 1249

Total PSMs (peptide sequence matches): 22811

\section{5. +carbamoylation +deamidation}

Total high-quality proteins: 1249

Total PSMs (peptide sequence matches): 21711 


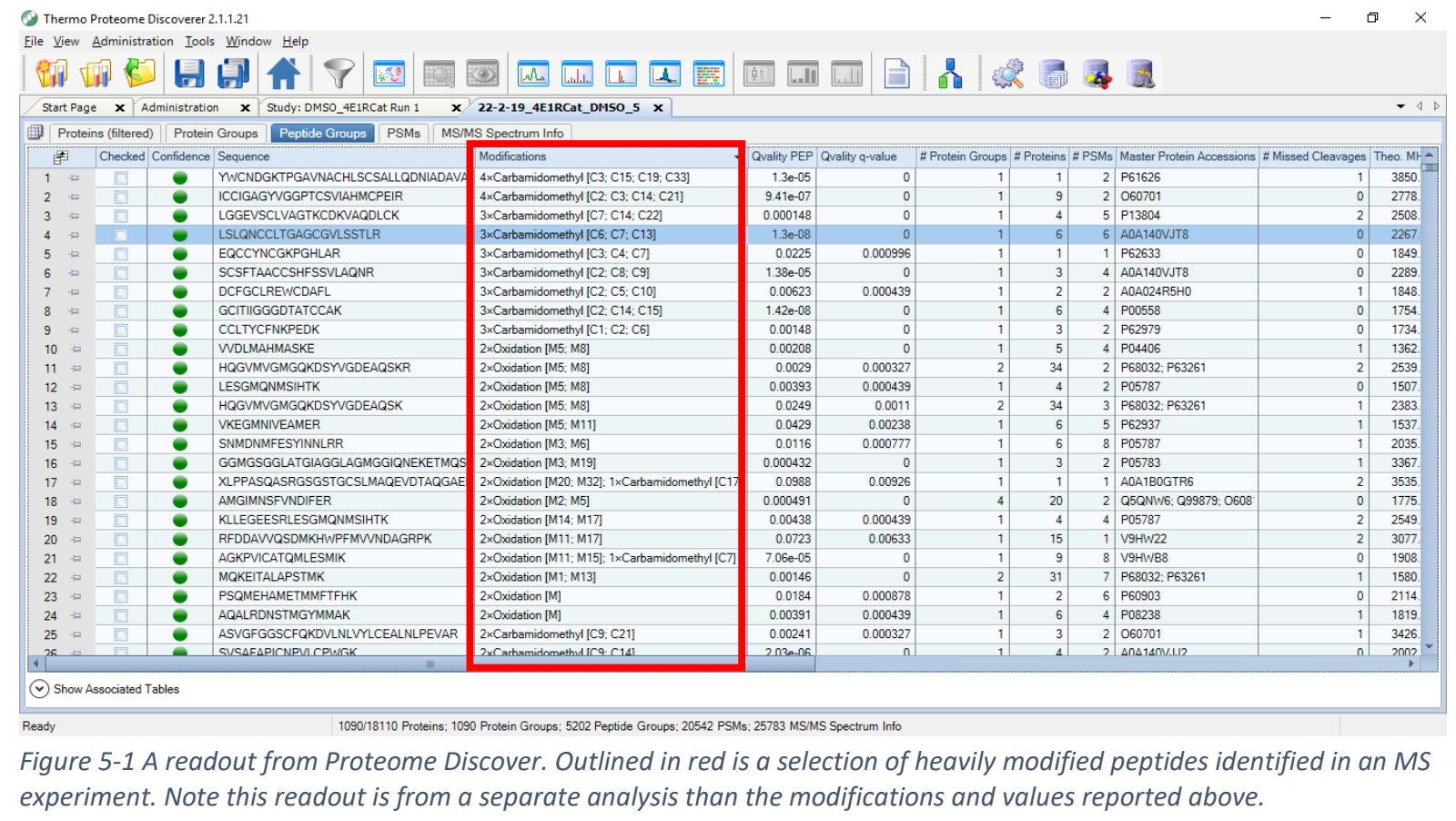

\subsubsection{Label-free protein quantification from MS/MS spectra}

The desire to use label-free tools for protein quantification offers benefits that include limited manipulation of the original biological sample over the course of an experiment, saving time and reagents. The techniques for quantification based on MS spectra have expanded and many of these are available with their own strengths and weaknesses depending on the nature of the experiment. MS Spectra were analysed using licensed software (Scaffold 4) using in-built label-free methods of quantification. Total ion current (TIC) is a no-nonsense method that is very robust, however, iBAQ which has been developed with an ability to correct for proteolytic fragments from a given protein also looks to be an effective method. Spectral counting employs a similar alignment method as used for calculating mRNA abundance in an RNA-seq experiment, where the 'reads', in this case validated peptides are aligned with a known protein sequence. This method was ignored as there are well known biases, as larger proteins will generate more peptides and it is affected by how the machine is running on a given day. This research follows on from previous research performed by our group in which TIC and iBAQ were both used, and thus both techniques were used for protein quantification from the MS spectra. If a significant difference became apparent between the two quantification methods, it was noted and assessed.

\subsection{TIC - Total ion current quantification}

TIC is a label-free quantification tool and an extension of spectral counting. It is reliable and more robust to some of the problems that plague spectral counting as a quantification technique. For a TIC quantification the average of the TIC for all of the MS/MS spectra that identify a protein is used as a quantitative measure. With the TIC method each spectral count is assigned a unique abundance value (Asara, Christofk, Freimark, \& Cantley, 2008).

\subsubsection{2 iBAQ - Intensity based absolute quantification}

$\mathrm{iBAQ}$ is a relatively new quantification tool that works very well for protein quantification with various methods of correcting for bias. The central concept of iBAQ is taking the sum of peak intensities of all peptides matching to a specific protein this value is taken and divided by the number of theoretically observable peptides. This process is corrective and aims to control for the capacity of larger proteins to have their peptides overrepresented in a sample. The process of 
figuring out which peptide fragments are theoretically possible is aided by knowing the digestion enzyme, in this case trypsin cutting at lysine/arginine (unless followed by proline). The values from this iBAQ method are an accurate proxy for protein levels (Schwanhäusser et al., 2011).

\subsubsection{Statistical techniques for dealing with false positives}

Multiple testing correction with Bonferroni usually makes all results insignificant. False discovery rate algorithms are necessary to figure out what is worth exploring further, FDR as a concept is interesting, it invites the possibility of encountering false positives (e.g. discovering a protein not present in a sample) while attempting to minimise false negatives (e.g. not recognising a correctly identified protein as significant). As an example, below in the first treatment data run through the mass spectrometer. Aiming to identify a change in protein expression between the 4E1RCat treated cells and the DMSO treated cells a list of 1052 proteins was identified. Of these, at 5\% FDR no significant change was noted, at $10 \%$ FDR, 2 protein changes became significant, at $15 \% 20$ protein changes became significant (18 proteins increasing in expression and 2 decreasing) and at 20\% FDR 22 changes were identified as significant. At an FDR of $15 \%$, it would be expected that $15 \%$ of the changes identified as significant are erroneous, corresponding to 3 insignificant changes being falsely identified.

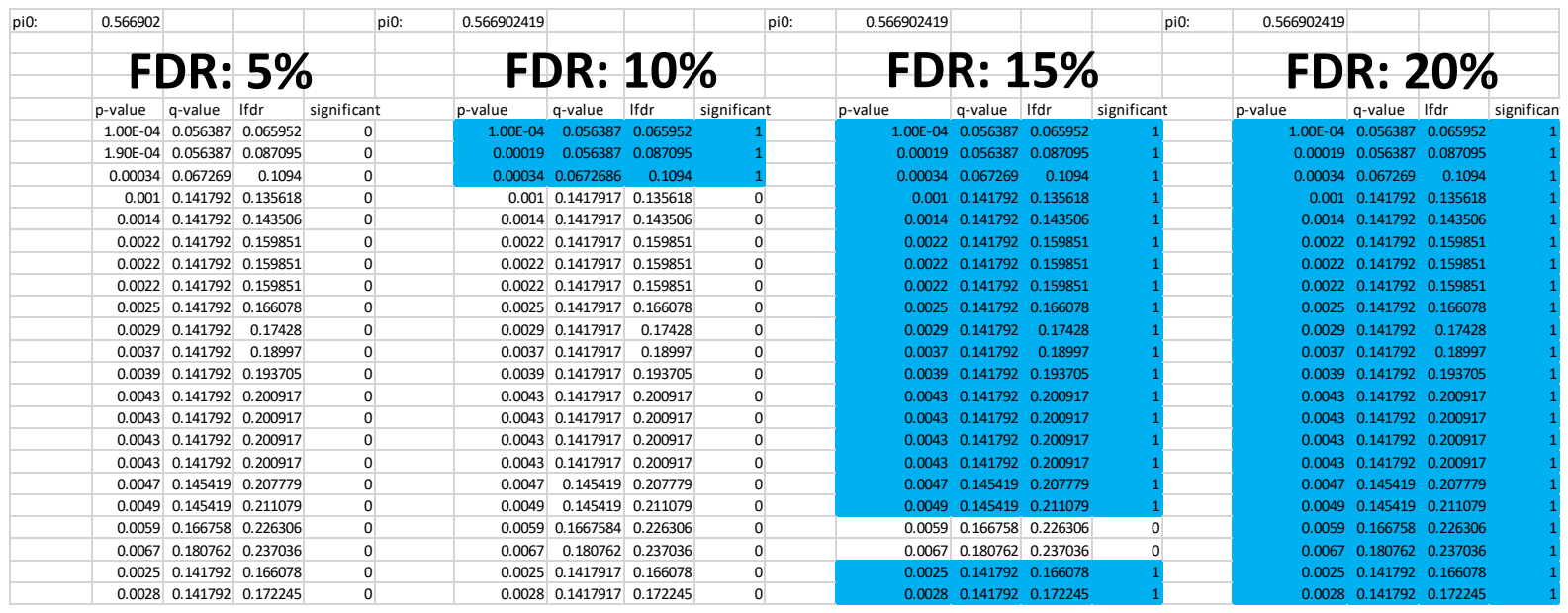

Figure 5-2 A table of 1052 proteins identified between the 4E1RCat and DMSO treatment conditions (only showing the top 22). Four FDR stringencies, 5,10,15,20\% were applied. Cells highlighted in blue were scored as significant by the FDR calculation. FDR calculations were run through a web service retrieved from http://qvalue.princeton.edu/

\subsection{Multiple testing correction}

With the rise of computational biology and the mountains of data modern biologists are capable of generating, the use of multiple testing correction is essential. High-throughput experiments, including RNA-seq studies and proteomics aimed at identifying global protein expression changes between treatments, are examples of molecular biology experiments where multiple testing correction is used. The problem being solved by multiple hypothesis testing is that when tests are done enough, chance plays a part in determining whether significant findings will be deemed insignificant and vice versa. As an example, if 100 proteins from experimental data are known to be unaffected by a treatment, i.e. levels don't change between a treatment and a control, at a defined statistical confidence cut-off, of $\alpha=0.05$, five of these 100 by pure chance will have $p$-values that would encourage rejection of the null hypothesis (i.e. give a response that would be assumed to indicate a change, or a failure to change, between treatments). The risk of falsely discovering a protein is something accepted as part of a proteomics experiment, as the goal is to generate leads which can be validated by molecular biology techniques. The problem with this is that t-tests 
individually are frequently undertaken at an $\alpha=0.05$, generally speaking. However, as the number of tested outcomes gets higher the absolute number of false positives increases. The t-tests performed for a given protein are a comparison between the untreated control and the treatment condition, if the distributions of peptide 'counts' or another quantitative measure between control and treatment are the same, the p-values would be uniformly distributed (Figure 5-3A). Conversely, if there are changes in a quantitative measure between treatment and control, the distributions would overlap less and thus in terms of $p$-values the distribution would be skewed towards lower values (figure 5-3B). As an example, in a case where most protein levels are unaffected by drug treatment, and a small proportion are affected, statistically you have a case where the affected proteins which will have $p$-values skewed and closer to 0 and the $p$-values from the unaffected proteins which will have uniformly distributed $p$-values between 0 and 1 comingling in the same analysis. Multiple testing corrections like the Benjamini-Hochberg attempt to address this by essentially overlaying the histograms by summation of the $p$-value distributions of treated and untreated conditions. Then drawing a line across the uniform portion of the distribution crossing into the affected and unaffected proteins the $p$-values above the line are true-positives. Based on the general FDR of 0.05, less than $5 \%$ of the significant results will be false positives (figure 5-3C). The False Discovery Rate (FDR) approach attempts to control the proportion of false discoveries in the results. A decision is made beforehand to accept a proportion ' $\alpha$ ' of false discoveries, then a threshold is calculated in a way that ensures that the global FDR is expected to be at worst equal to $\alpha$.
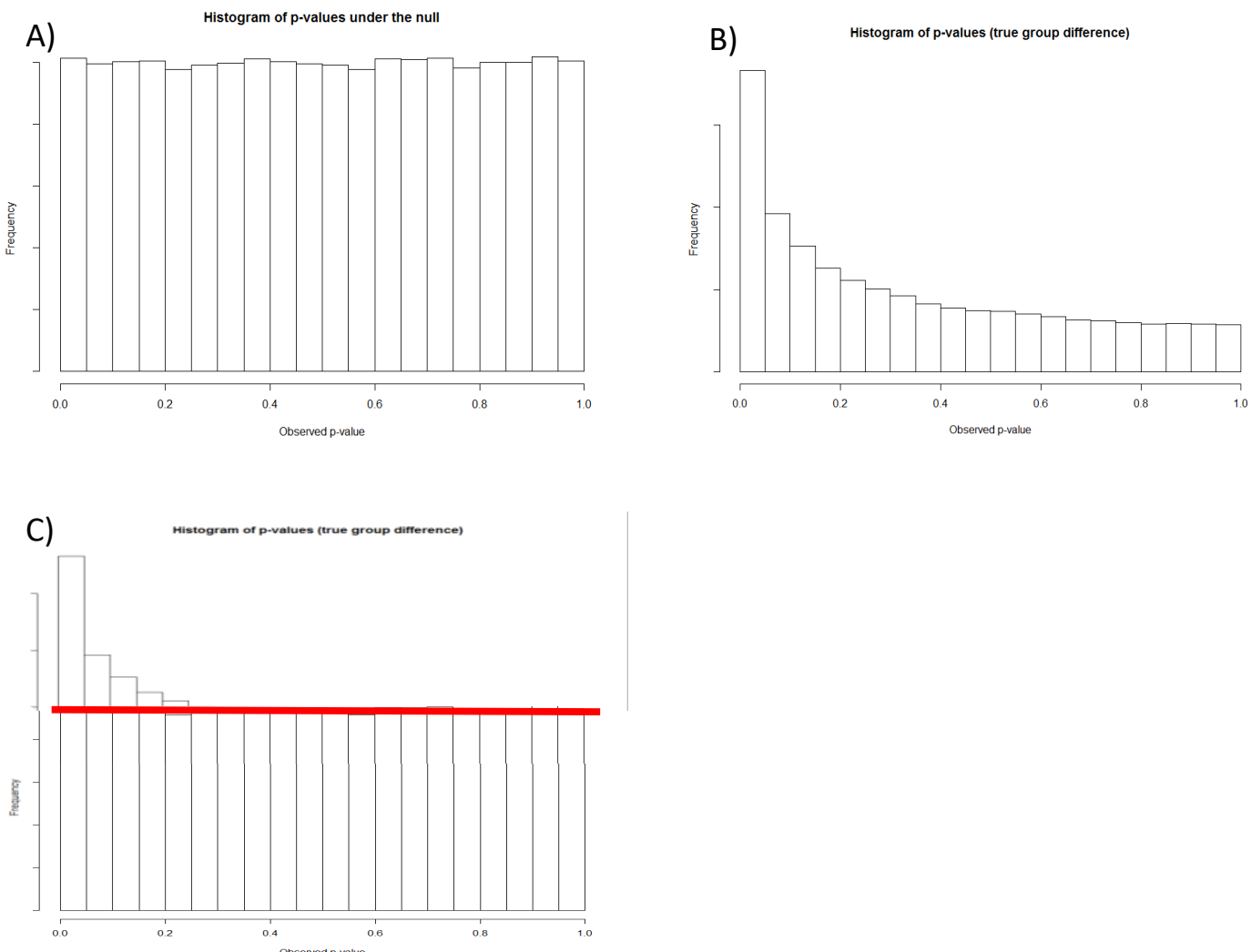

Figure 5-3 A) An example of an even statistical distribution, if peptide 'counts' or another quantitative measure between control and treatment are the same, the p-values would be spread uniformly. B) If there are changes in a quantitative measure between treatment and control, the distributions would overlap less, and p-value distribution would be skewed towards lower values. C) An example of a distribution in which both A) and B) are present in the same analysis, a BenjaminiHochberg-type correction is applied by the red-line across the uniform portion of the graph. 


\subsection{False discovery rates (FDR)}

False discovery is a statistical concept that involves accepting an erroneous outcome or 'false positive' as a true positive outcome. In statistical analyses, there is an inherent risk that a type 1 error, a false positive, or a type 2 error in which a statistically significant result is discounted as insignificant, a false negative. The goal of FDR is to minimise false negatives, at the cost of introducing false positives into an analysis at an unspecified rate, in this study the FDR is set at $10 \%$. While the analysis now captures most if not all of the potentially significant findings, they are now interspersed with erroneous results which cannot readily be separated from correctly identified results.

http://qvalue.princeton.edu/- is a web-based server that performs a Benjamini-Hochberg-like multiple testing correction in which the FDR rate can be specified.

\subsection{Decoy protein database strategies for FDR estimation}

Decoy protein databases are an empirical strategy for estimating FDR, the basic concept involves generating sets of 'decoys' which are a set of distinct generated peptide/protein sequences that don't correspond to actual peptides/proteins. These decoys are seeded among experimentally identified peptides and the final number of decoys that make it through in the list of identified peptide spectrum matches (PSMs) is an accurate way of empirically determining FDR. Generated decoys are generally beholden to criteria that try to ensure:

1. Similar amino acid distributions as target protein sequences.

2. Similar protein length distribution as target protein sequence list.

3. Similar numbers of proteins as target protein list.

4. Similar numbers of predicted peptides as target protein list.

5. No predicted peptides in common between target and decoy sequence lists.

(Speicher, 2010)

There are two core strategies for generating decoys that can satisfy the above criteria, the first and simplest is a 'reversal' strategy that involves reversing the sequence of a peptide/protein to obtain a new entity with the exact constituent amino acids but a non-existent (typically) counterpart in the realm of protein/peptide sequences. The second, a 'stochastic' strategy employed by search engines like Mascot, one of the primary protein validation search engines used for protein identification in this thesis. This involves the generation of proteins in a pseudorandom manner, proteins are random in some aspects while following amino acid bias patterns or even using a Markov chain model to better imitate real proteins by mimicking micro-patterns such as single or double amino acid repeats or highly basic/acidic regions (Speicher, 2010).

Multiple testing is a fundamental component of modern biology, and statistical-mathematical and empirical-computational methods in the proteomics field are used to ensure that the number of false-positives and false-negatives are minimised within a given statistical analysis. Corrections like the Bonferroni and Benjamini-Hochberg adjustments are common in this space, modern proteomics also uses an empirical-computational approach to false discovery that involves seeding an analysis with known decoys to model the FDR rate. These two approaches are fundamental to this thesis and have been employed extensively on the path from raw LC-MS ${ }^{2}$ through to PSM identification, to lists of identified and quantified proteins and finally to lists of proteins up or downregulated in response to a treatment. 


\subsection{Insights from gene ontology and STRING analysis}

The FDR adjustment used alongside Scaffold's existing multiple testing corrections was deemed too stringent as it removed all significant hits from the three main treatment conditions, cycloheximide, ribavirin and 4E1RCat. The DMSO condition however, gained an additional 163 hits at a 10\% FDR adjustment through the Princeton Edu web server (Migliaccio, Rotondi, \& Auricchio, 2006). In response to this, it was decided that on the whole, the $10 \%$ FDR adjustment was too stringent for the main treatments, where the focus on a concentration that produced a small growth defect resulted in few hits. In contrast, it was too generous for use in the DMSO control analysis, which had many hits and accepting a 10\% FDR would mean including a large number of false positives. Therefore, the analyses discussed here use the up and downregulated hit list from the Scaffold software, depending only on its multiple testing corrections to limit false positives. Gene ontologies (GOs) are categories of processes, functions, and locations that use standard terms to describe the characteristics of biological systems. An ontology analysis moves the analytics away from what the individual protein or gene specifically does in a molecular sense and attempts to generate new insights by contextualising the specific molecular mechanisms of each individual protein responding to a treatment into more general processes. For example, a DNA helicase specifically unwinds DNA, but is in a more general sense involved in replication. Although at the molecular level the DNA helicase melts and annuls complementary base pairing, its function as a helicase is in the context of providing access to polymerases and other DNA replication proteins to the now single stranded DNA which is important for the replicative process. The use of standard GO terminology ensures it is possible to group proteins together under common ontological terms, and therefore also to analyse protein changes under the three GO domains - Biological Process, Molecular Function, and Cellular Component. Ontological analyses are useful for understanding what processes, functions and cellular locations are associated with a proteome response to a treatment. There are additional ways of analysing proteome responses, beyond the $\mathrm{GO}$ terms. These include investigation of changes in sets of proteins that relate to specific pathways, which can be obtained through pathway databases, such as KEGG (Tanabe \& Kanehisa, 2012). There are also databases, such as the CORUM database, which can be interrogated to identify whether responding proteins are found in the same complex. Another analysis tool used is STRING (Szklarczyk et al., 2017), this is a visualisation tool that constructs a network of protein nodes based on the confidence with which the nodes interact. Interaction networks can be based on a range of features, including appearance in the same publications, correlation in databases (such as GO and KEGG) and co-expression. This latter analysis reveals clusters of proteins in a set that have been shown to be co-transcriptionally regulated. Proteins in a co-expression network are generally involved in the same biological responses, and these networks can be a useful tool, in addition to GO term analysis, to understand the proteins that appear in the proteome response data. In our analyses, due the small number of hits, in the form of upregulated and downregulated proteins, analyses were sometimes done with a combination of up and downregulated hits to improve the confidence relating to affected processes. The STRING analyses were constructed in this manner, with the exception of the up and downregulated proteins in the DMSO condition, where no benefit was achieved by doing analyses on the combined hits. It is important to note that the apperance of a protein in the $\mathrm{GO}$ analysis does not indicate that it will form part of a cluster in the STRING diagrams. All the hits from the Scaffold analysis were included in the GO and STRING analyses. However, in the STRING analyses if the protein did not have partners from the same analysis the node was not included in the STRING output. 


\subsubsection{Cycloheximide (69 hits)}

In the work presented here, cycloheximide is used as a control to evaluate which changes are occurring due to general translational stress, and which are specific to the compound under study. Cycloheximide is expected to decrease protein synthesis uniformly across the protein expression profile of the cells treated at $I C_{10}$. We may also see a stress response signal due to stalled translation at the ribosome.

GO term enrichment analysis reveals that spliceosomal process and ribonucleoproteins are upregulated in the cycloheximide treatment (Table 7). This assessment is supported by the STRING analysis of the combined up and downregulated proteins (Figure 5-4). This shows a spliceosomerelated protein cluster as well as a ribosomal protein cluster. The upregulation of the ribonucleoproteins by cycloheximide is expected as it directly affects protein synthesis at the level of the ribosome. It is likely that cycloheximide is removing a portion of the protein output capacity of the cells and the cellular response is to produce more ribosomes to counteract the loss. The spliceosomal process could also be explained by this observation as new ribosomes will need to be built by the remaining functional ribosomes in the cell and mRNA is the precursor to both the rRNA and ribonucleoproteins.

Proteins involved in ubiquitination and protein degradation via the proteasomal process are downregulated.

Interestingly, associated with the proteosomal components in the cluster diagram are proteins involved in ubiquitination and energy metabolism in the form of enzymes involved in the production of NADPH and ATP. The proteosome is energy dependent and requires a ubiquitination signal for degradation of proteins.

The CORUM and KEGG analyses showed no changes in the upregulated hits condition. In the downregulated hits condition, three complexes showed up in the CORUM analysis: PA28-20S proteasome, the OTUB1-UBC13-MMS2 complex and the MCM4-MCM6-MCM7 complex. These are involved in proteasomal degradation, ubiquitination and DNA replication respectively. These three processes being downregulated in response to protein synthesis inhibition is logical. The balance of protein synthesis is disturbed, and the cells are responding by downregulating the degradation of proteins via ubiquitination and the proteasome. Under normal circumstances proteins will be replaced by protein synthesis. Since cycloheximide has perturbed protein synthesis, downregulating the turnover of proteins is a necessary adaptation to preserve remaining functional proteins. The decrease in DNA replication may be a generalised stress response to the toxicity of cycloheximide, an instruction to cease or lower replicative efforts. In the combined analysis of up and downregulated proteins the CORUM analysis pulled out an additional complex that is not involved in proteasomal degradation that didn't show up in either of the separate analyses, the SF3b complex which recognises the branch point adenosine of pre-MRNA as part of the splicing process (Rakesh, Joseph, Bhaskara, \& Srinivasan, 2016). In the combined condition, the KEGG analysis showed two pathways termed proteasome (as expected from the GO and STRING analyses) but also arginine biosynthesis. Response of the arginine biosynthetic pathway to translational stress has been observed previously (Venturi et al., 2018). 


\section{Cycloheximide}

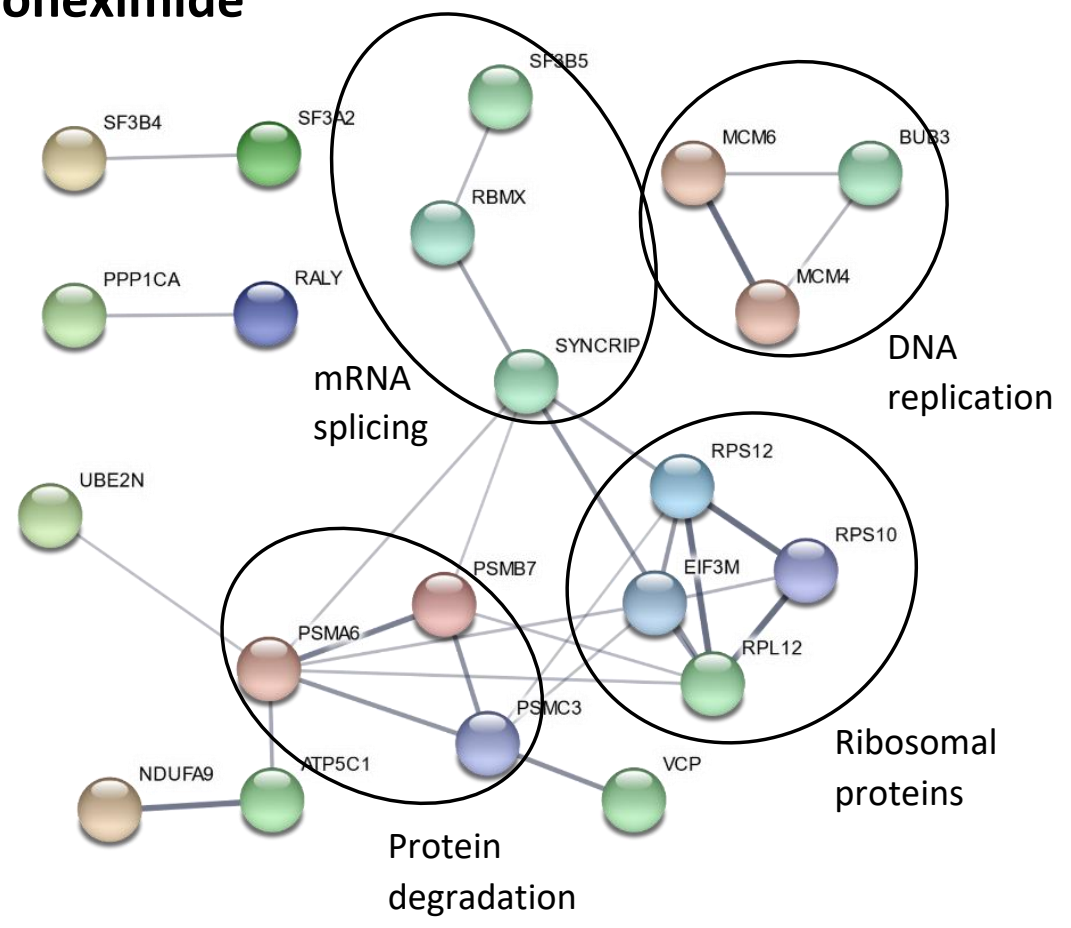

Figure 5-4 STRING diagram of proteins expression change in response to low-dose treatment with cycloheximide

\subsubsection{Ribavirin (83 hits)}

The two main processes seen in the upregulated hits are cell adhesion/cadherin and proteasomal proteins involved in protein degradation.

Interestingly far more proteins seem to be downregulated in response to ribavirin treatment, and subsequently more processes are affected. Three main processes seem to be ribosome biogenesis, protein targeting to the endoplasmic reticulum (ER), and RNA metabolism. The CORUM analysis of the downregulated hits showed two complexes, the Nop56p-associated pre-rRNA complex presumably a complex involved in ribosomal biogenesis (Hayano et al., 2003), and cytoplasmic ribosome. The KEGG analysis also notes that the ribosome is downregulated. Taken together these results suggest that ribavirin is influencing the ribosome and its biogenesis.

No new clusters or processes appeared in the combined analysis. The enrichment for ontologies relating to cell-cell adhesion and cadherin binding is unique to the results found with ribavirin and points to a function of ribavirin that is not related to translational inhibition. This is not unexpected, as ribavirin has been noted to have multiple cellular targets. The observation of a downregulation in cadherin binding proteins is consistent with a recent publication which suggests this may be part of the mechanism through which ribavirin can protect against viral infections, at least in combination with an interferon treatment (Rendón-Huerta et al., 2013). 


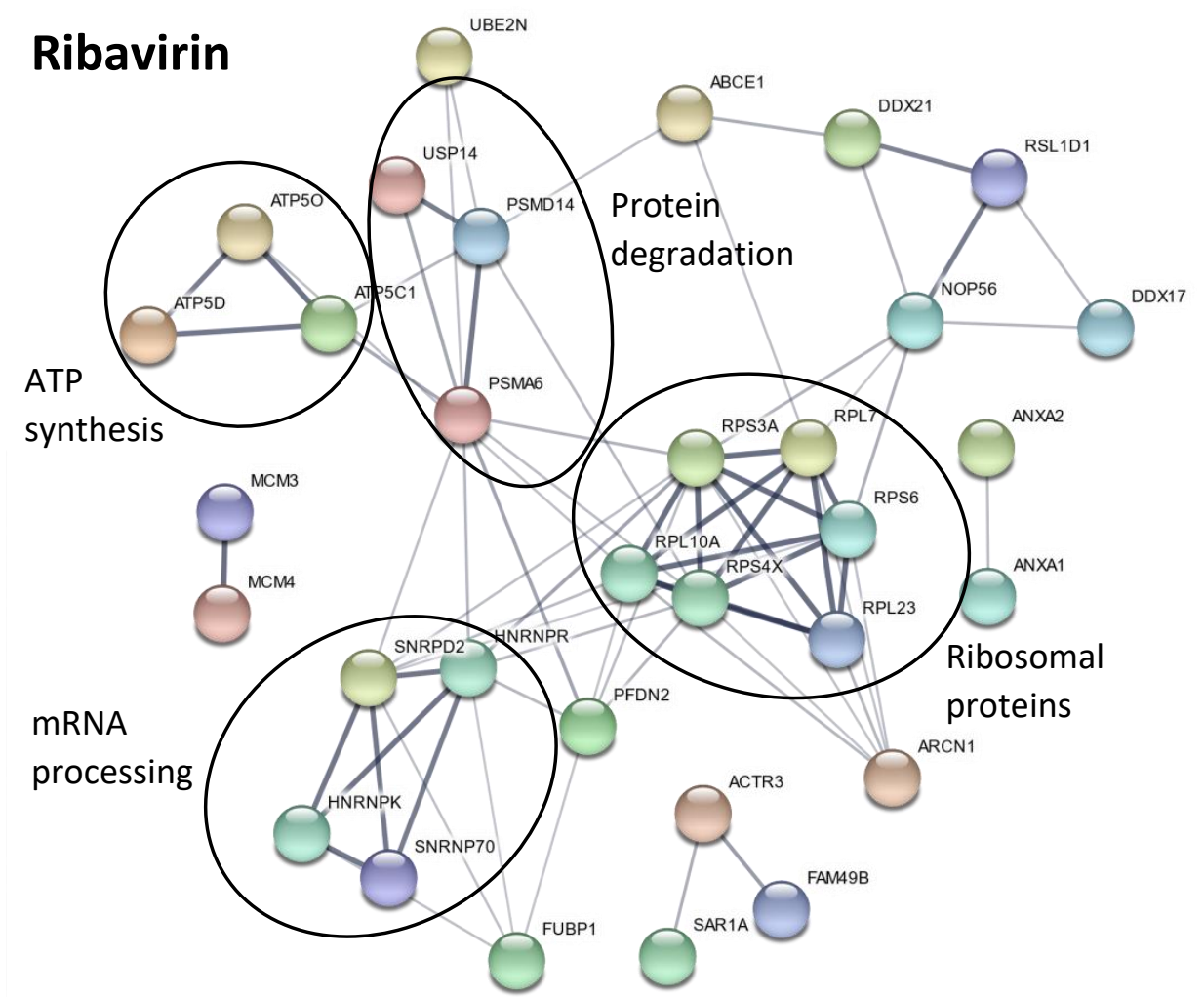

Figure 5-5 STRING diagram of proteins expression change in response to low-dose treatment with ribavirin

\subsubsection{E1RCat (47 hits)}

Upregulated proteins and processes in response to 4E1RCat involve ribosome biogenesis, protein targeting to the ER, and RNA metabolism.

The downregulation change in response to 4E1RCat seems to be minimal, the affected processes involve the mitochondrial proteins HADHB (involved in beta-oxidation) (Naiki et al., 2014), ATAD3A (a heavily expressed mitochondrial membrane protein involved in nucleoid organisation, and various aspects of growth and metabolism) (Li \& Rousseau, 2012), and TFAM (a mitochondrial transcription factor) (Litonin et al., 2010). Also affected is the eukaryotic replisome, a complex required for rapid and accurate chromosome replication (Yeeles, Janska, Early, \& Diffley, 2017).

The CORUM analysis pulls out three clusters of which the cytoplasmic ribosome and 605 ribosomal subunit clusters are nested ontological terms. This means that the $60 \mathrm{~S}$ ribosomal cluster term contributes to the significance of the cytoplasmic ribosome term. Also, in this analysis is the Nop56passociated pre-rRNA complex, as noted for ribavarin. The CORUM analysis indicates effects on ribosome biogenesis. The KEGG analysis confirms this with ribosome being the only process identified. The effects on mitochondrial proteins and processes and effects on eukaryotic replication were unexpected. No additional insights were obtained through the combined analysis. 


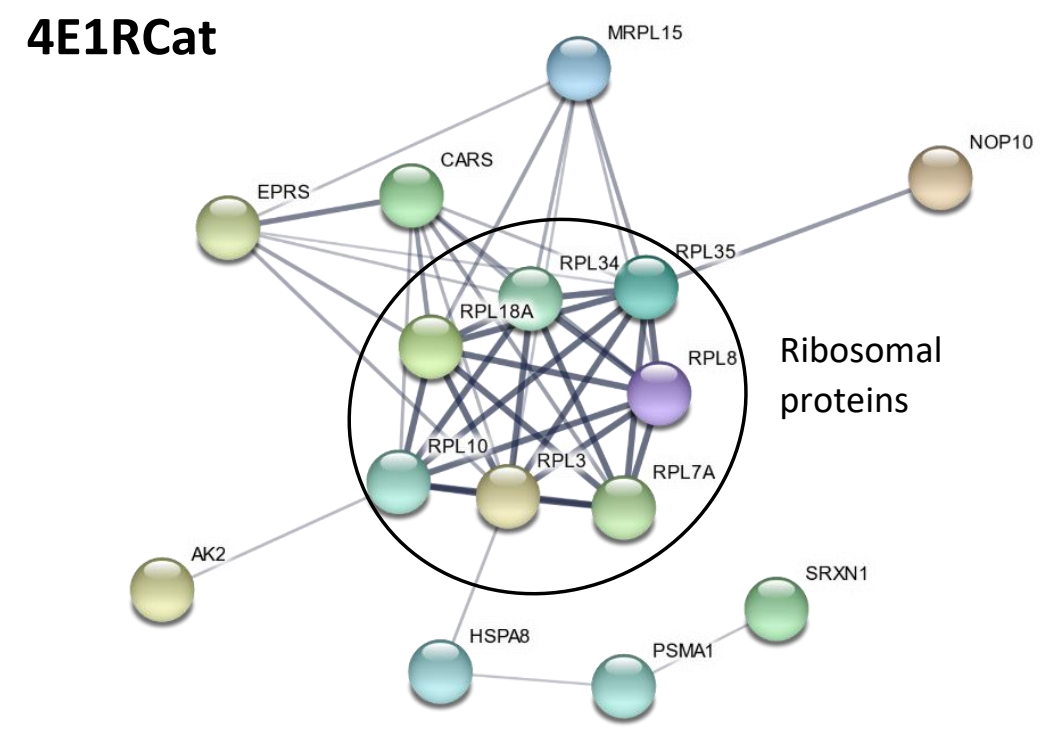

Figure 5-6 STRING diagram of proteins expression change in response to low-dose treatment with 4E1RCat

\subsubsection{Comparisons between treatments}

The number of hits aligns with the expectations from the drugs in terms of molecular specificity within the cell. Ribavirin, the drug with the most expected interactions within the cell (1.2.4.2), has the largest number of hits, whereas 4E1RCat, with only two molecular interactions (1.2.4.1), has the least hits. cycloheximide is middling. This may be superficial, but it is interesting to note, nonetheless. Unique to the 4E1RCat treatment is the downregulation of mitochondrial nucleoid proteins and the eukaryotic replisome. For the ribavirin treatment, upregulation of cell adhesion molecules and proteins associated with the cell adhesion process is also unique. It is noticable that responses to ribavarin are in the opposite direction to those observed in other treatments. For example, the decrease in ribosome and ribosome biogenesis is in contrast to the increase in proteins associated with these ontologies found for cycloheximide and 4E1Rcat. A trend of converse regulation between ribavirin and 4E1RCat, protein targeting to the ER is a downregulated process in the RBV treatment and an upregulated process in the 4E1RCat treatment. In the same vein proteasomal proteins and processes are seen in the cycloheximide and RBV, however in cycloheximide they are downregulated and upregulated in ribavirin this process is upregulated. Finally, RNA metabolism is another process that is downregulated in the RBV treatment but upregulated in the 4E1RCat treatment. 


\subsubsection{DMSO (364 hits)}

The intention of the DMSO treatment was as a control, and so it is not a treatment aligned with the aims and objectives of this thesis. It was required, due to the low solubility and low activity of 4E1Rcat, which combined to require a high DMSO concentration in its treatment. However, the sheer strength of the response to $1 \%$ DMSO in the HT29 cells is intriguing. The $1 \%$ DMSO treatment condition roughly corresponds to a growth inhibition of between $30-40 \%$. In the context of this study this would be considered a medium-high dose treatment, which explains the magnitude of hits seen in the DMSO condition. I strongly think that 4E1RCat is having a molecular effect on the cells. Observation of cell pellet sizes post-drug treatment indicates that 4E1RCat is contributing to lowering proliferation, as cells in the DMSO control run alongside 4E1RCat had noticeably smaller pellets, which were stained red interestingly (4E1RCat is red in solution). Although appropriate DMSO controls were run in tandem with the 4E1RCat treatment it may not be possible to deconvolute the effects that DMSO is exerting on the proteome, it may be possible that effects we see in the 4E1RCat condition have been tweaked by the action of DMSO. Although it is possible to overlay the proteomic effects of the treatment and control and subtract the DMSO controls' proteomic effects from those exerted by 4E1RCat. However, this might not be convincingly possible, it may be that some of the effects seen in the 4E1RCat treatment are a result of the combined efforts of the two compounds. The decision to analyse the DMSO condition proved worthwhile as some of the processes affected by such a general toxic agent appeared in the treatment conditions by compounds that were expected to be far more selective comparatively.

DMSO has wide-ranging effects on cells, upregulated processes ranked by significance include targeting to the ER (localisation and targeting), translation (initiation, peptide biosynthesis and ribosome biogenesis), RNA catabolism (cellular nitrogen catabolism, aromatic compound catabolism, nuclear base-containing compound catabolism and heterocycle catabolism). Some minor upregulated processes include DNA conformation change and energy metabolism.

The processes that respond to DMSO through protein downregulation include RNA metabolism, translation, splicing, protein folding and exocytosis.

DMSO is likely to have general toxic effects on the cell, perhaps interestingly in the context of the other treatments, a high proportion of the processes seen in the other treatments are also seen in the DMSO condition. Localisation to the ER, effects on translation, RNA metabolism and catabolism, splicing and minor effects on energy metabolism are seen on aggregate in the three treatment conditions. In this sense, DMSO appears to be delivering responses typical of a translation inhibitor. This was an unexpected finding, and not anticipated at the outset of the study. However, investigation of the literature revealed a 2019 paper which investigates the effects of DMSO on cells (Verheijen et al., 2018). The study included a proteomic analysis, for which data (presented in the supplementary information Tables 3 and 4 ) is consistent with the results presented here. Although analysed through a different process, focusing on pathways from the ConsensusPathDB with the Reactome database, the proteomic data shows exceptional q-values (down to $10^{-49}$ ) associated with translation and the ribosome, making these the strongest pathway hits in their proteomic study. 


\section{DMSO}

\section{upregulated}

hits

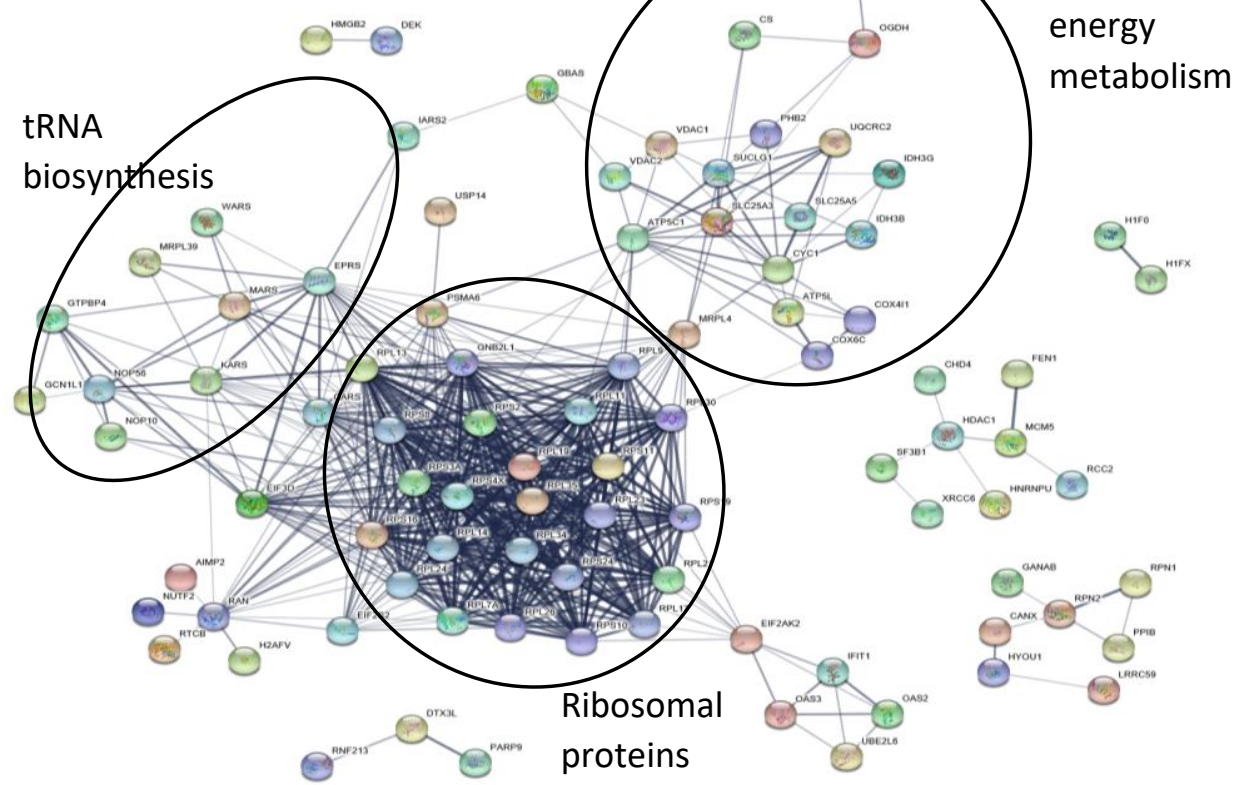

Figure 5-7 STRING diagram of upregulated proteins expression change in response to treatment with DMSO at $1 \%$, equivalent the concentration in the 4E1RCat top

\section{DMSO}

downregulated

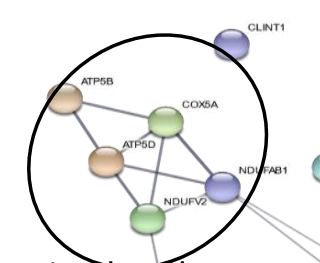

Mitochond

rial

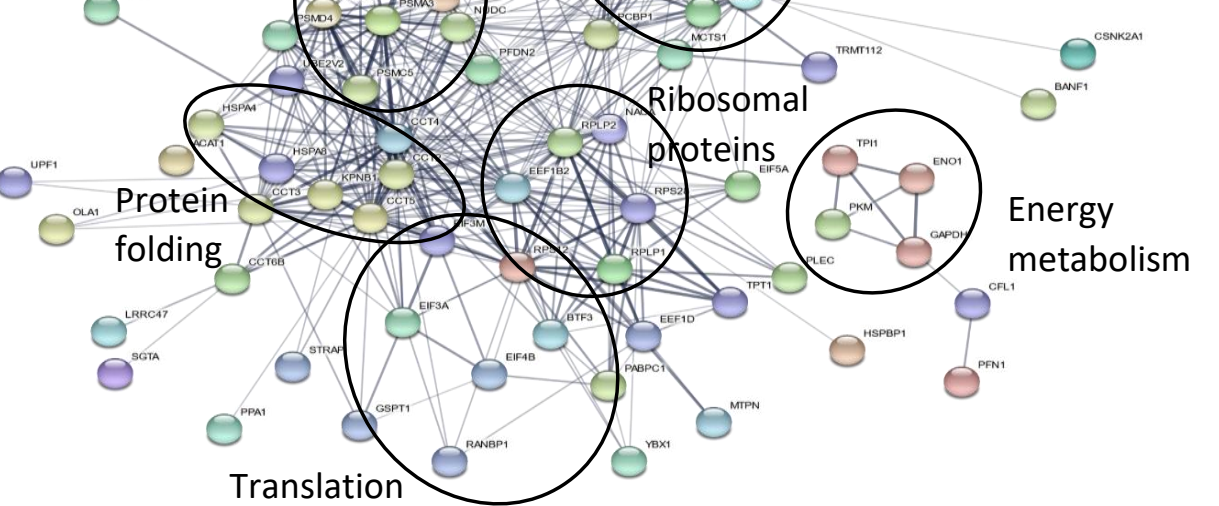

Figure 5-8 STRING diagram of downregulated proteins expression change in response to treatment with DMSO at $1 \%$, equivalent the concentration in the 4E1RCat top dose 


\subsubsection{Summary of insights from GO and STRING analysis of treatments}

In summary, the drug treatment effects on the HT29 cells largely result in ontological enrichments related to processes that they are the directly targets of the treatments. In terms of named processes, the treatments caused many of the same effects, which include ribosomal processes, splicing, protein degradation, targeting to the ER and energy metabolism. It seems highly likely that proteomic outcomes in response to each compound are a direct result of the perturbation of the molecular process in combination with general toxic responses to treatment and the subsequent effects on the cell. Some unique responses do appear in the ribavirin treatment in the form of effects on cell adhesion and expression of cadherins in ribavirin. Likewise, in the 4E1RCat treatment condition mitochondrial nucleoid and the appearance of the eukaryotic replisome are unique. The revelation that DMSO causes cellular responses that are consistent with protein synthesis was unexpected. Furthermore, it brings into doubt the proteome responses observed in response to 4E1Rcat. This compound required high concentrations of DMSO to solubilise it at a concentration of $1 \%(v / v)$ DMSO sufficient to cause a growth defect in the HT29 cells at. Even though the up-and down-regulated proteins associated with the 4E1Rcat treatment have been determined in relation to the DMSO control, it is difficult to confidently rule out that noted enrichments might arise from experimental artifacts. Conversely, the low number of varying proteins and enriched proteins found with the 4E1Rcat treatment may arise simply because 4E1Rcat-induced changes are being masked by the cellular response to DMSO.

\subsection{General discussion}

\subsubsection{The purpose and power of a whole-proteome experiment}

Mass spectrometry is an excellent tool for investigating protein expression at a global level and prospecting for interesting biological outcomes and generation of hypotheses to further explore and validate using molecular biology techniques. Generally, biases and enrichment from sample handling and data analysis are minimised to ensure that any enrichment noticed in a given proteomics experiment are a consequence of biological activities and processes, rather than a consequence of a given method of protein isolation and analysis. Proteomics often begins without a prior hypothesis in mind and is a prospective analysis tool. Therefore, the software we use to analyse our spectral data and the methods of cell lysis and protein precipitation are likely to bias the analysis. However, these biases can be mitigated by a thorough understanding of the analytical tools, and the enrichment outcomes of a given wet-lab methodology. An example of a false enrichment discovery by members of our lab group involved noticing that treatments from HT29 and HL60 cells treated at $\mathrm{IC}_{10}$ values with PatA were heavily enriched for extracellular exosomes. Although initially interesting, retrospectively the extraction methods removed many membrane-bound proteins and enriched for cytosolic proteins; extracellular exosomes are derived from the membrane and cytosolic components, which could explain this enrichment.

\subsubsection{The significance of 'low dose'}

Previous research suggests that PatA has its anti-cachectic effects at low doses, well below antitumour dosing. Since the nature of PatA's anti-cachectic effects are still poorly understood and may involve the elF4F complex, it is appropriate when targeting another component of the same complex to treat it at the same dosing scheme as this will help clarify whether it is low dose elF4A inhibition mediating the effects, or whether the complex as a whole plays a role in cachectic reversal.

\subsubsection{E1RCat and separating the DMSO effects from drug treatment effects}

The effects of compounds on proliferative or metabolic activity of human cancer cell lines is a way to establish the potency of a drug. Cell lines have variable responses to the anti-proliferative effects of 
a given treatment. The MTT assay is a mainstay in drug discovery, the principle of this assay is the conversion of 3-(4,5-dimethylthiazol-2-yl)-2,5-diphenyltetrazolium bromide (yellow) to its corresponding formazan (purple) by actively metabolising cells. This assay was used extensively in this thesis as a tool to assay the anti-proliferative effects of compounds selected for this study. Dimethyl sulfoxide (DMSO), the solvent used to reconstitute the drugs used in this thesis has demonstrable anti-proliferative effects. Data was collected to establish the effects of the vehicle on the cell lines used. DMSO alone was shown to have an $\mathrm{IC}_{1}$ of $0.151 \%$ ( $\mathrm{v} / \mathrm{v}$ in buffer) in HT29 cells and $0.621 \%$ (v/v in buffer) in HL60 cells. DMSO controls were included in the analysis to address the effects DMSO has on cells. A concentration of $0.1 \%$ ( $/ / v$ in buffer) has been established as having negligible cytotoxic effects, evidenced by the literature (Timm, Saaby, Moesby, \& Hansen, 2013) and backed by MTT assays performed over the course of this thesis. DMSO controls were only relevant to the 4E1RCat treatment conditions, where the 100-fold drop in concentration from the stock concentration in $100 \%$ DMSO to $1 \%$ DMSO in the top dose, which corresponds to a $\sim \mid C_{10}$ treatment, is not enough of a dilution to avoid the cytotoxic effects of DMSO. For CYCLOHEXIMIDE and ribavirin, the ability to dissolve these drugs at $50 \mathrm{mM}$ and their cytotoxicity being significantly higher than $4 \mathrm{E} 1 \mathrm{RC}$ at mean that in the process of diluting the drugs to their appropriate $\mathrm{IC}_{10}$ values in treatment conditions, the DMSO concentration is low enough to not warrant controlling for the effects of DMSO. As an example, the $\mathrm{IC}_{10}$ of ribavirin in $\mathrm{HT} 29$ cells of $11.1 \mu \mathrm{M}$ is more than 1000 -fold lower than the $50 \mathrm{mM}$ starting stock concentration. Cycloheximide with an $\mathrm{IC}_{10}$ of $0.0427 \mu \mathrm{M}(42.7 \mathrm{nM})$ is also below 1000 -fold more dilute than the starting stock. The $\mathrm{IC}_{10}$ of DMSO generated from an assay of HT29 cells treated with DMSO was $0.4 \%$ by volume. This contrasts with the top dose of 4E1RCat treatment, which was selected as the $\sim \mathrm{IC}_{10}$ treatment value which has a DMSO concentration of $1 \%$. These findings conflict, as by my treatment data an $\mathrm{IC}_{10}$ for DMSO treatment is at a lower percentage than used in the top dose in the 4E1RCat treatment condition used as an $\mathrm{IC}_{10}$. One could interpret this as 4E1RCat being protective against the effects of DMSO, however, this is unlikely. Another interpretation is that since the 4E1RCat data was assayed on six separate occasions, that it is the more reliable collection of data. Whereas the dose response to DMSO, was initially completed as an exploratory experiment and subsequently an $\mathrm{IC}_{10}$ was generated. The assay was only replicated two times, if replicated a few more times the $\mathrm{IC}_{10}$ may shift. Over the course of this project, it has been repeatedly noted that 4E1RCat is not particularly cytotoxic to cells (see Figure 4-3). When dissolving 4E1RCat, significant solubility issues were encountered, consistent with some online resources which showed conflicting solubility values for dissolution in DMSO. Efforts to dissolve the drug by sonication were unsuccessful, the drug eventually became soluble at a concentration of $4 \mathrm{mM}$, about 10 -fold lower than the $50 \mathrm{mM}$ stocks of RBV and CYCLOHEXIMIDE. Early attempts to solubilise at higher concentrations were motivated by the low cytotoxicity expected from 4E1RCat. These concerns manifested in the form of a struggle to get 4E1RCat to kill cells even at the top dose of 40 $\mu \mathrm{M}$, at this dose the DMSO concentration is $1 \%$ making it hard to determine whether 4E1RCat is having any effects or whether the effects of DMSO are being observed. RBV by contrast was soluble up to $180 \mathrm{mM}$ and demonstrated by dose response curves to be significantly more cytotoxic by comparison. As mentioned in the introduction 4E1RCat only has 2 molecular interactions, with 4EBP and elF4E, in this sense it is pharmacologically 'clean' (1.2.4.1). However, due to its low cytotoxicity (see Table 1 ) and low solubility (see 4.2 ), the approximate $\mathrm{IC}_{10}$ value is influenced by the DMSO vehicle which is at $1 \%$, this may confound results. The appropriate DMSO controls have been generated in response to the conditions necessary for 4E1RCat to be useable as a treatment. Despite this, we may expect that 4E1RCat will have a small uniform lowering effect on protein synthesis in line with its molecular function as an elF4E inhibitor.

\subsubsection{Ribavirin, a 'dirty' drug with multiple interactions}

It has been noted in the literature that Ribavirin, a commercially available clinical drug, has a suite of molecular interactions at a range of concentrations. Ribavirin mainly targets enzymes involved in metabolism, but also polymerases, consistent with its status as a guanosine mimic. This is further 
evidenced by its ability to be misincorporated into viral mRNA at millimiolar concentrations by viral RNA-dependent RNA polymerases, Crotty et al suggest that the effect of this on viral replication is an increase in mutation rates in response to misincorporation of ribavirin into the viral genome to a catastrophic level, however a caveat to this is that the researchers used ribavirin at 20 times higher a concentration than clinically relevant doses, at $100 \mu \mathrm{M}$ (Crotty, Cameron, \& Andino, 2001). This interaction with RNA-dependent RNA polymerase is not the central mediator of its broad anti-viral activity. This has been attributed to inhibitory effects on inosine monophosphate dehydrogenase (IMPDH) by ribavirin 5'-monophosphate, leading to depletion of intracellular pools of GTP (Leyssen, Clercq, \& Neyts, 2006). Strong evidence for this model of interfering with viral replication, is that treatment with ribavirin results in a 4-log reduction in viral RNAs which can be partially reversed by the addition of guanosine. Researchers noted, interestingly, that despite suspected involvement of IMPDH in ribavirin's anti-viral activity, mycophenolic acid, another IMPDH inhibitor had no anti-viral effect (Lanford et al., 2002). As mentioned previously (1.2.4.2), despite conflicting findings (Westman et al., 2005; Yan et al., 2005), ribavirin has been confirmed to interact with elF4E at micromolar concentrations (Kentsis et al., 2005, 2004). The conflicting results were suggested to be a result of free-eIF4E (often referred to as apo-elF4E) being sensitive to structural changes at $\mathrm{pH}$ changes between 7.5-8. Some backing for this is the observation by Westman et al (Westman et al., 2005) that ribavirin bound to elF4E at 2-4 orders of magnitude lower than originally reported by Kentsis et al (Westman et al., 2005), suggesting that buffer conditions in the conflicting papers could be lowering the capacity of eIF4E to bind ribavirin. Ribavirin is unlikely to exert its molecular effects in its unmetabolized form, ribavirin is metabolised in the liver and intracellularly. Since the intracellular metabolism is the form relevant to a cell culture, this will be the focus. Ribavirin is the substrate of adenosine kinase which converts ribavirin into ribavirin monophosphate (RMP), which in turn is the substrate of monophosphate and diphosphate kinases which respectively dephosphorylate, and triphosphorylate RMP into ribavirin triphosphate (RTP). In most cell types RTP dominates at concentrations 20-100 times that of RMP (T. Page \& Connor, 1990). Viruses function within the cell and the cellular machinery is co-opted for their replicative processes, thus the relevance of such an in-depth assessment of ribavirin's mode of action in an anti-viral setting is relevant to the effects it may have on cellular processes. With such a wide variety of molecular targets at a variety of concentrations, it is important to acknowledge this when treating cells, as the effects on the proteome may be confounded by the other interactions within the cell. The effects of ribavirin are slightly less certain in comparison to cycloheximide or 4E1RCat due to the variety of molecular targets it is known to interact with, refer to section 1.2.4.2. We expected that there would be a general lowering effect on protein synthesis as some proportion of elF4E will be competitively inhibited by ribavirin. There may be other effects as ribavirin lowers the GTP pool within the cell which may lead to a cellular response for guanosine producing enzymes or enzymes involved in generating or using GTP.Although other processes, such as those associated with cell-cell adhesion, were found, the relatively clean ontological enrichment profile of ribavirin for processes associated with translation validate its potential for studies of this kind.

\subsubsection{Cycloheximide - control protein synthesis inhibitor}

Cycloheximide is a eukaryotic protein synthesis inhibitor used extensively in molecular biology to stop the cellular production of protein. Cycloheximide was selected as a control compound due to its known protein synthesis inhibition by blocking translation at the ribosome (Schneider-Poetsch et al., 2010). More importantly cycloheximide inhibits all protein synthesis in a non-selective manner, thus it was an appropriate tool to use as a positive control in these experiments as the goal is to tease out any specific effects of elF4E inhibitors from the change in the proteome that could be a result of a biological response to translation stress, giving a skewed or selective effect on protein expression. 


\subsubsection{Regulatory network influence on translational output}

It is not apparent that the magnitude of change any individual protein can make to a cell is in proportion to its abundance. In fact, the opposite can be true. If you take a set of 100 proteins randomly selected from a proteome, it is likely that some of these proteins will have structural, enzymatic, or regulatory roles within the cell. However, the majority of the proteins in which change is detected are likely to be high abundance structural and enzymatic proteins. Transcription factors are the most extreme example of a proteins with extremely low abundance but correspondingly extreme effects on gene expression and protein synthesis in the cell in response to growth factors and the cellular stresses within an environment. Transcription factors, despite being encoded by $6 \%$ of the genome (Barabási, Gulbahce, \& Loscalzo, 2011) and so the second largest group of genes, are generally the lowest in abundance by far. Evidence of this being that only $5 \%$ of all TFs have been purified and characterised (Ngagore et al., 2013).

Whilst transcription factors provide one example of how an abundance change in a protein can cause broad change within a cell, they are not alone in having this property. Barabási etl al describe non-transcription factor related abnormalities in an insightful way - "The impact of a specific genetic abnormality is not restricted to the activity of the gene product that carries it, but can spread along the links of the network and alter the activity of gene products that otherwise carry no defects." (Barabási, Gulbahce, \& Loscalzo, 2011). This insight can be extended to the outcome of translational inhibition. For example, pateamine $A$ is suspected to give selective translation inhibition of transcripts with specific 5' UTR structures in a manner similar to rocaglates (Iwasaki et al., 2016). Due to the nature of cellular regulatory networks, if the translationally repressed gene product is part of a network - and especially if it is a key regulator - its down-regulation can have flow on effects on protein expression lower down in the pathway. This observation can be used to potentially trace the effects of a treatment and its effects on proteins that are identified through a proteomics experiment and potentially finding a regulator, such as a transcription factor, or set of processes responsible for the changes seen in protein expression between treated and untreated cells.

\subsubsection{The search for 5' UTR motifs}

It has been observed that motifs in the 5' UTR sequences are often involved in mRNA regulation. This can happen in two ways. In the first, the sequence itself is a recognisable element that can be bound by a regulatory protein that can either increase or decrease some aspect of expression of these mRNAs. An example of this is the 5'-Terminal Oligopyrimidine tracts (TOP) motif present in $30 \%$ of transcripts in actively growing mammalian cells (Pichon et al., 2012), the TCT motif is also a requirement for transcription for the majority of the TOP containing mRNAs (Pichon et al., 2012). The second way is by the motif generating a higher order secondary structure that indirectly interferes with translation by enforcing a requirement for helicase activity prior to expression, as mentioned in the introduction (see 1.2.1). Even for a transcript with a $5^{\prime}$ UTR with minimal secondary structure, translation can be severely inhibited if elF4A is not present. Although the intention initially was to explore $5^{\prime}$ UTR motifs to see if there were transcripts that respond to elF4E treatment in the manner observed with pateamine $A$ (previous work by our lab group) and presumably part of the puzzle that explains why pateamine A can rescue cachexia at low doses. We have not ruled out that there are eIF4E responsive transcripts. At a glance our eIF4E treatment data does not present enough proteins that cannot be explained by a direct biological response effects of ribavirin and 4E1RCat treatment. In terms of running motif enrichment analyses, statistically speaking small numbers of proteins, and their transcripts are likely to generate false leads by way of randomness. We have not been exhaustive in our separation of biological responder transcripts and elF4E inhibitor sensitive transcripts on the current analyses approach in the interest of time. 
However, it is in many ways justified as a replacement drug for 4E1RCat would need to be found and a higher dose of ribavirin will need to be used to elicit a greater proteome response to justify running a series of motif enrichment analyses. As it currently stands, we do not have enough data to deny or confirm the existence of elF4E inhibition sensitive transcripts.

\subsection{Conclusion}

The findings in this thesis are unable to meaningfully test the notion that low dose elF4E inhibition has selective effects that mirror outcomes from pateamine A treatment. This arises because the eIF4E inhibitors used in this study each have problems associated with their use. Ribavirin has a suite of molecular interactions within the cell which can cloud the interpretation of the proteomic outcome in response to treatment. The major problem was the low efficacy of 4E1Rcat and its overlapping proteome response with the DMSO vehicle. The 4E1Rcat was chosen due to its reported selectivity for elF4E inhibition, and it was intended to use this to aid abstraction of elF4E-mediated effects from the proteome response of the polypharmacological ribavirin. Together, these create uncertainty in interpreting the proteome responses observed as being specific to elF4E inhibition. However, the ontological enrichments observed with ribavirin do support its use in ongoing studies. Furthermore, many of the proteins observed to decrease in abundance in response to elF4E inhibitors appear to be associated with the direct biological response to the known effects of the inhibitors, such as their effect on translation, as revealed by GO term and co-expression analysis. Relatively few protein changes are left to be explained in terms of translational repression based solely on a 5' UTR sequence motif. This small number coupled with the uncertainty of the origin of their translational repression led to the conclusion that sequence motif analysis would not be meaningful. The major outcomes of this work are, cycloheximide, ribavirin and 4E1RCat exert effects on the proteome consistent with their classification as translation inhibitors. An interesting finding comes in the form of DMSO our solvent of choices' effects on translation, seemingly consistent with a translation inhibitor at a growth inhibition of $30-40 \%$. Although there are a few examples of proteins and protein and protein clusters that may not be easily explained by a direct biological response of the cell line to perturbation by the treatments applied in this thesis. There is not enough information to ruled out the existence of elF4E sensitive transcripts. This study has paved the way for future experimentation with higher doses of ribavirin and a replacement inhibitor for 4E1RCat or another strategy to disentangle the effects of 4E1RCat from the DMSO vehicle it is dissolved in.

\subsection{Future directions}

Due to time restraints treatment data was obtained only in the HT29 cell line. The continuation of this project would involve confirming whether the observations seen in HT29 cells in response to treatment are mirrored in other cell lines. A mirror experiment in the HL60 cell line has been performed and these cells will be processed and run on the mass spectrometer to see if the data supports the findings seen in the HT29 cells, an experiment that unfortunately could not be accommodated in the timeline of this thesis due to instrumental down-time. Although initially the intention was to quantify proteins using both TIC and $\mathrm{BBAQ}$, in the interest of time TIC was used exclusively for protein quantification. Further research could make use of the robust iBAQ method and see if any new proteins appear in the analyses or other proteins disappear. The use of more potent or selective elF4E inhibitors would be an ideal way to confirm these results, especially if this allows avoidance of a solvent vehicle control such as DMSO. This may not be possible however, as 4E1RCat and another commonly used inhibitor, 4EGI-1, have comparable solubility. In fact, 4E1RCat seems to have the edge in this department. Based on the literature, 4E1RCat is the more frequently used of the two, and unfortunately alternative direct elF4E inhibitors may not yet exist. Another possibility, if it is impossible to find a stronger elF4E inhibitor would be to repeat the $\mathrm{IC}_{10}$ treatments with DMSO added to the cycloheximide and ribavirin treatments and perhaps pateamine $A$ as well to 
see if the proteomic effects of DMSO can be convincingly subtracted from a treatment. It would be worthwhile investigating the effects of ribavirin at a higher concentration to see if sufficient proteins can be downregulated, which fall outside the direct biological response to the translational stress, to investigate 5' UTR motifs associated with reduction in protein expression. Future research could also test the effects of hippuristanol or other available elF4A inhibitors on the proteome response to see if it matches the proteomic effects of pateamine A in HT29 and HL60 cells. Another possible avenue to explore could involve replicating the effects on muscle fibers, the muscle fibers responding to pateamine $A$ could be subjected to a proteomic analysis to gather more information about the specific effects of pateamine $A$ on the proteome of muscle cells, ideally from in vivo studies, and compared to the effect of ribavirin or another eIF4E inhibitor. 


\section{Appendices}

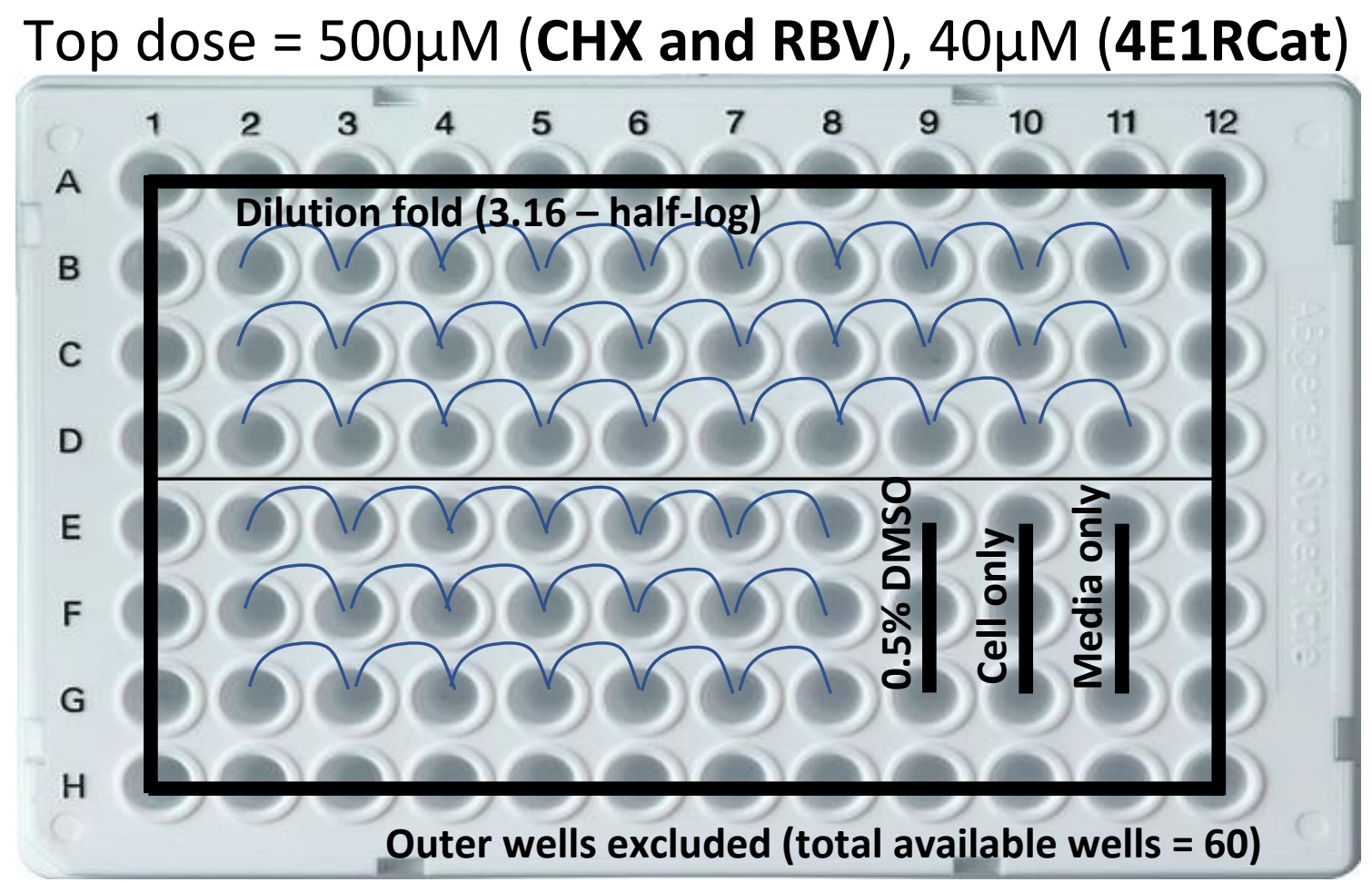

Figure 6-1 Plate plan example done in triplicate following a half-log (3.16) serial dilution scheme. DMSO controls were included at concentrations where DMSO is expected to influence cell growth. A media only blank and cell-only control are also included.

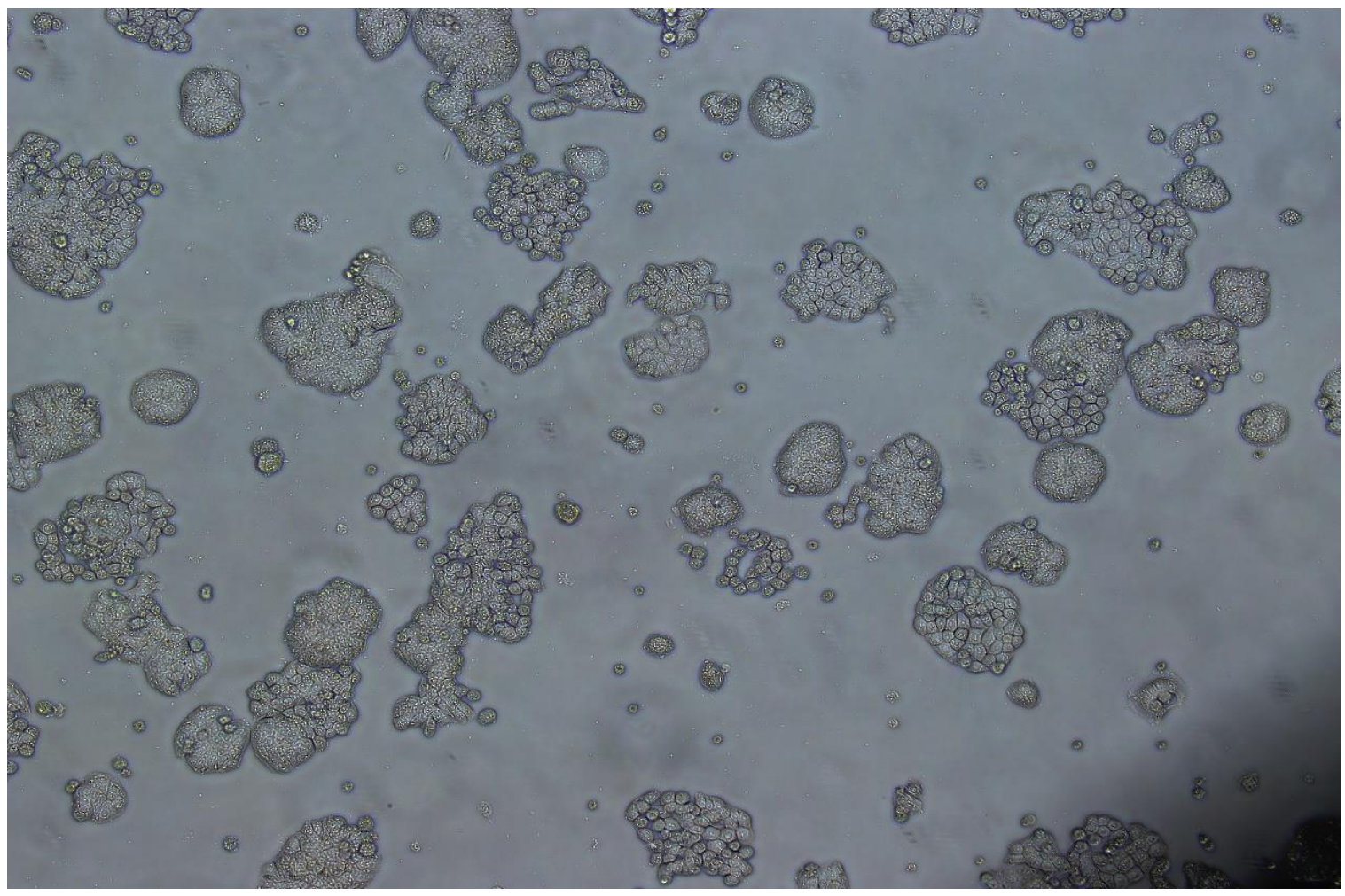

Figure 6-2 Shown at 10x magnification. Healthy cells from the original stock from 1999, after 1 passage. Note the the propensity of the cell line to form colonies, also note the healthy look and regularity of the glandular structures 


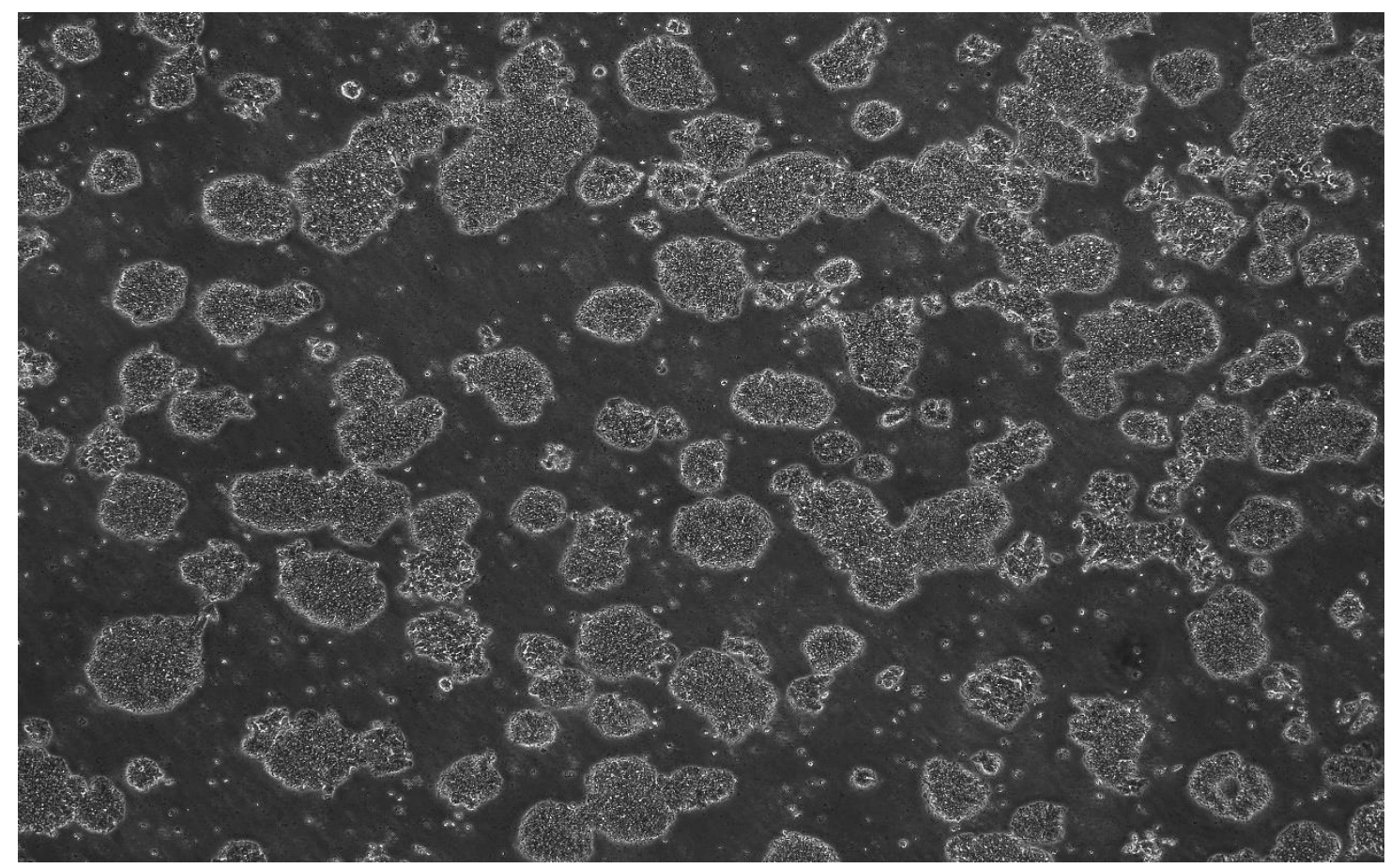

Figure 6-3 Shown at 10x magnification, HT29 cells grown in depleted acidic media at high confluence for 1 week. Note, that the glandualar structures are becoming jagged looking and the dead cells in suspension.

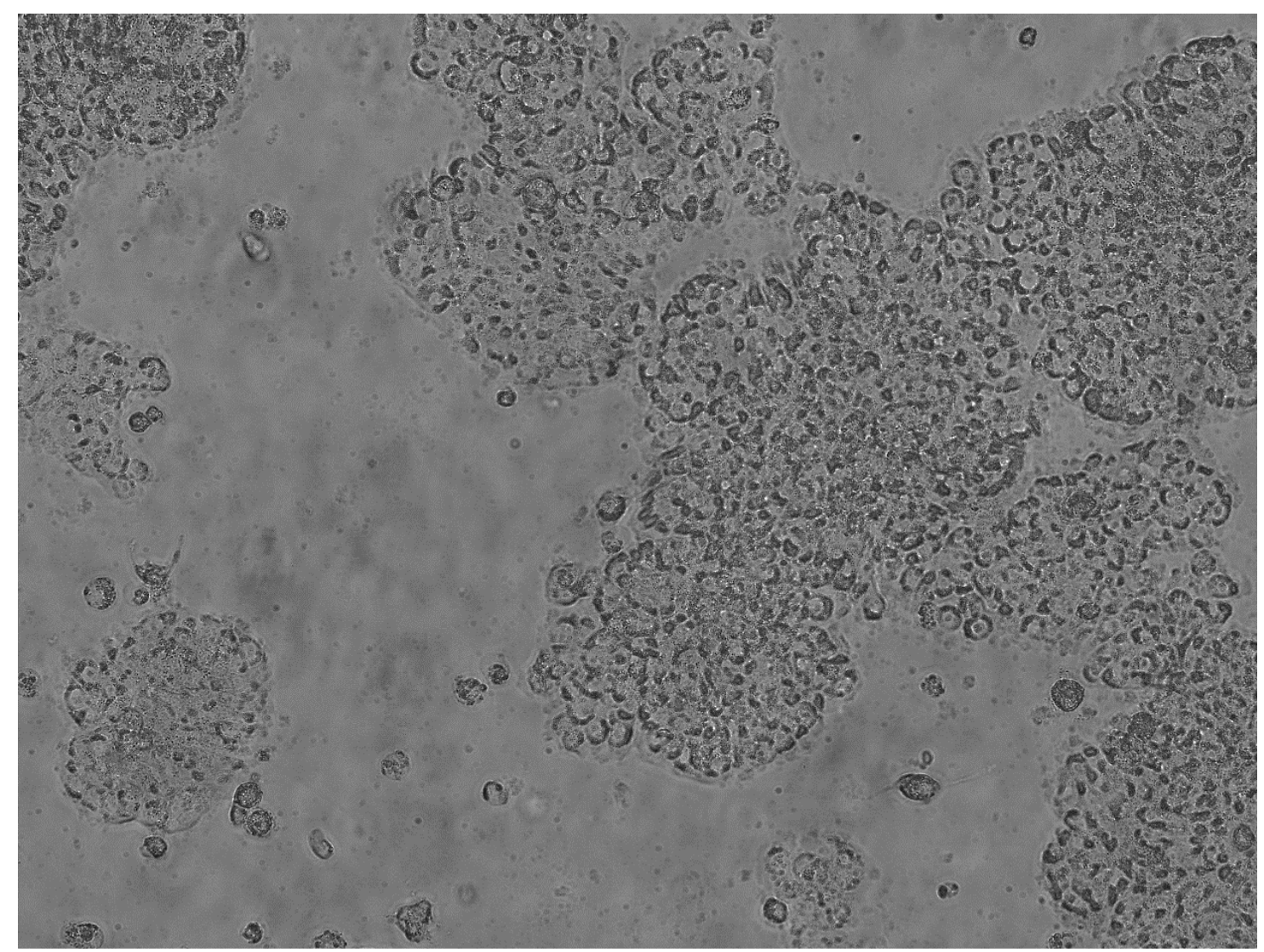

Figure 6-4 40x magnification - a closer look at cells from the same flask as figure 6-3. Note the debris and apoptotic blebs in the environment surrounding the cells 


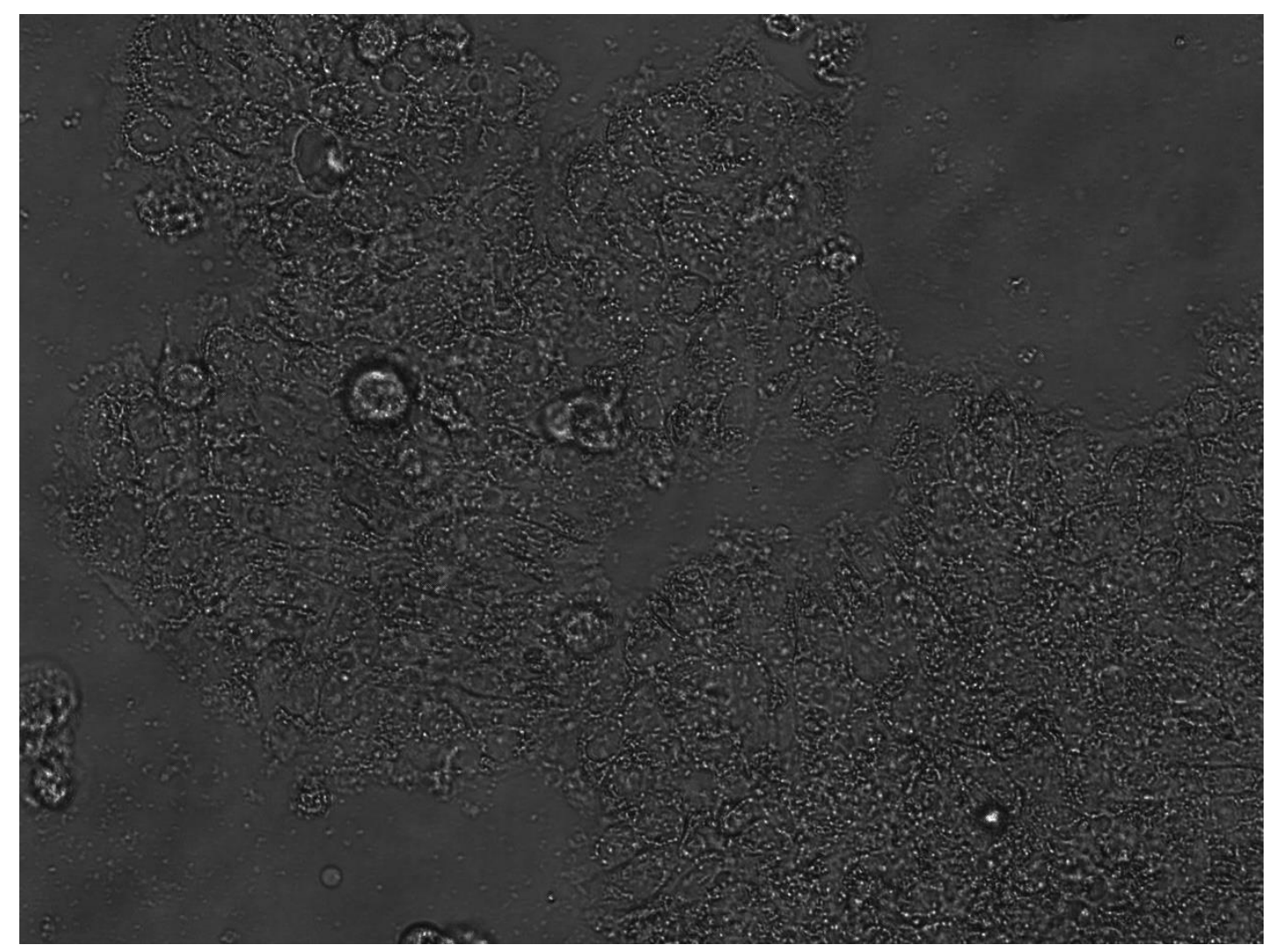

Figure 6-5 100x magnification - a closer look at cells from the same flask as figure 6-3. Note the number of apoptotic blebs in the environment surrounding the cells 


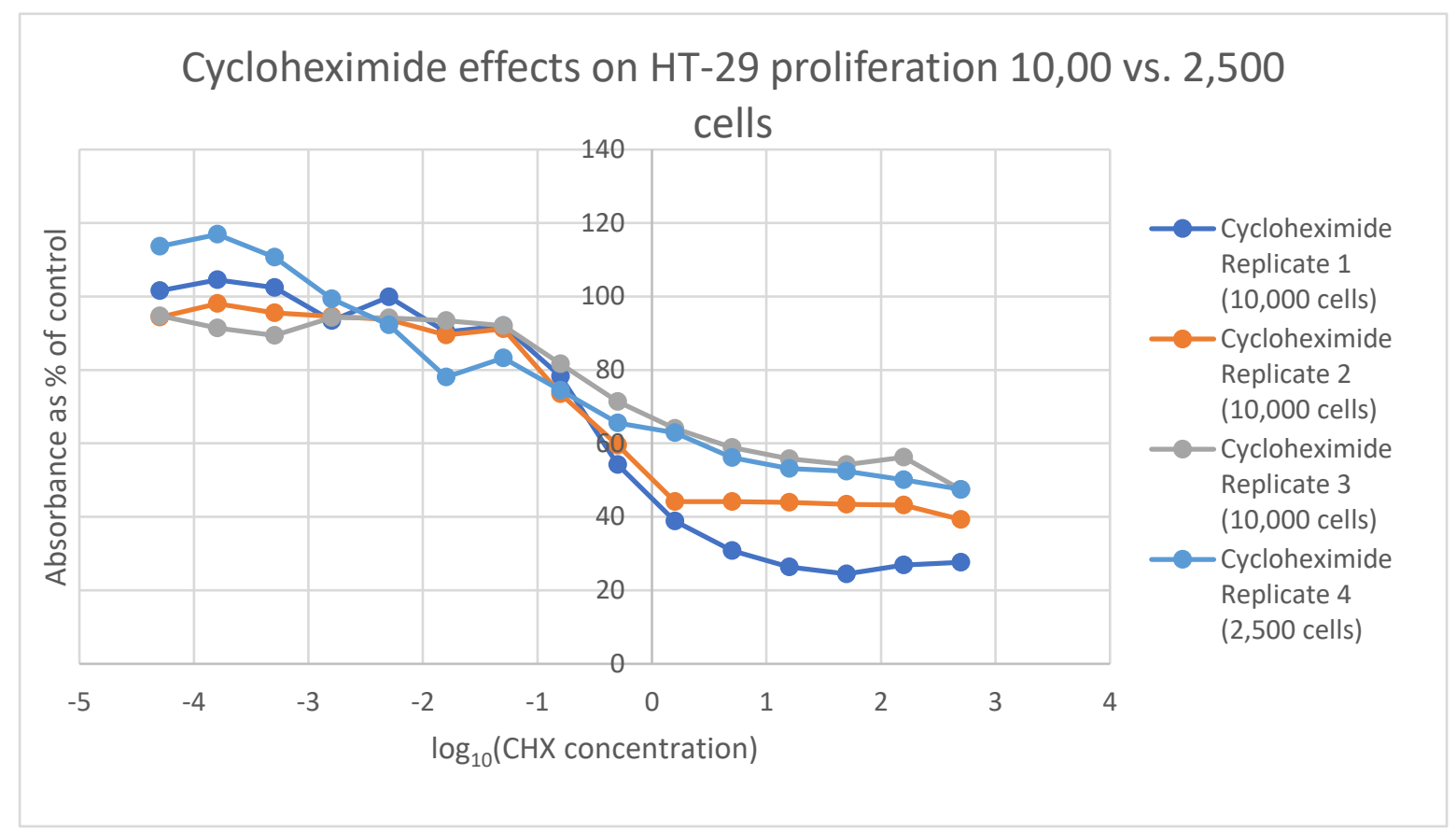

Figure 6-7 The effects of cell seeding density on proliferative signal from the MTT assay.

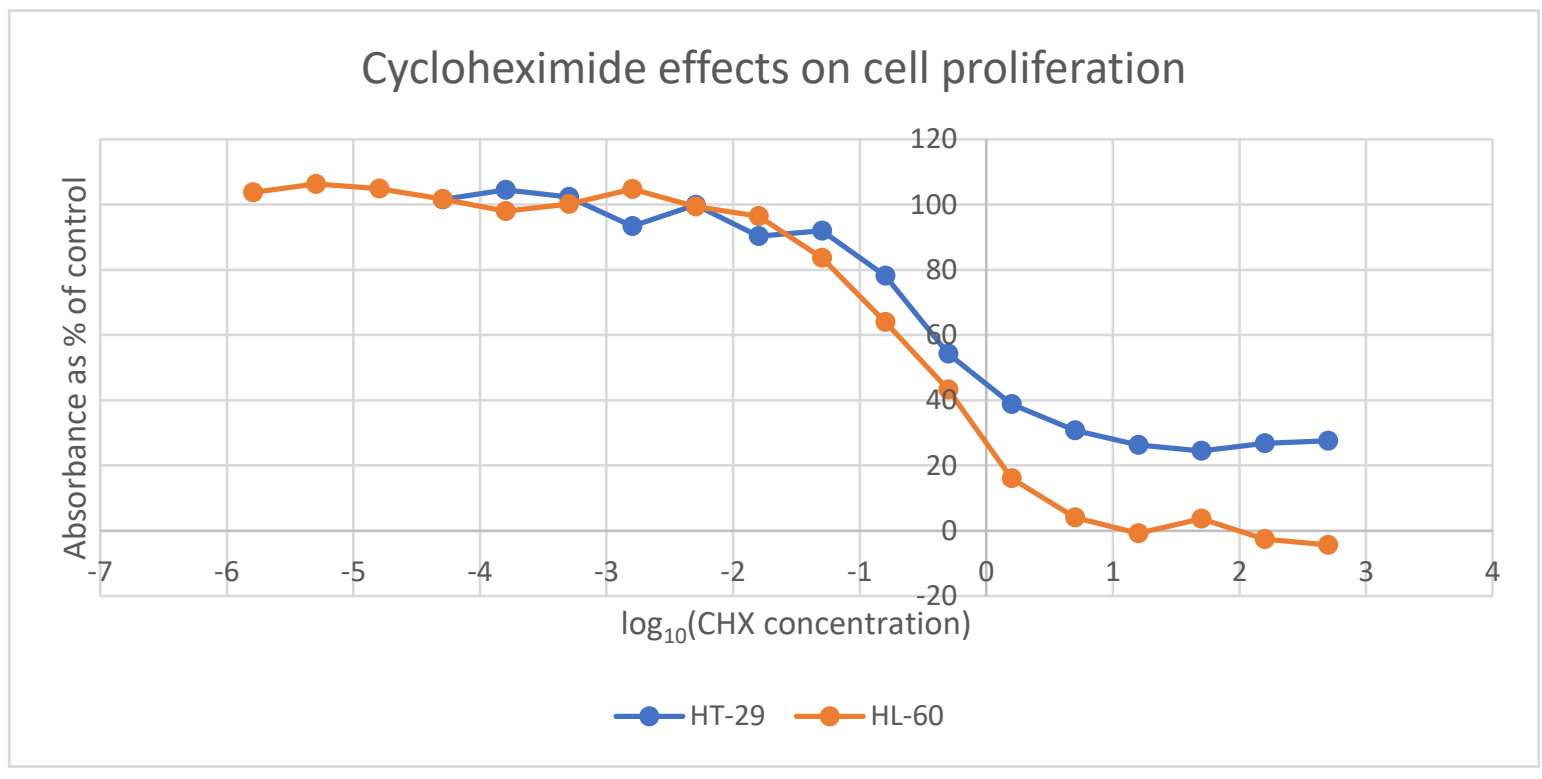

Figure 6-6 A comparison of the proliferative effects of cycloheximide on HT29 and HL60 cells. Note how in HT29 cells the araph does not level out at 0 , whereas the HL6O cells do. 


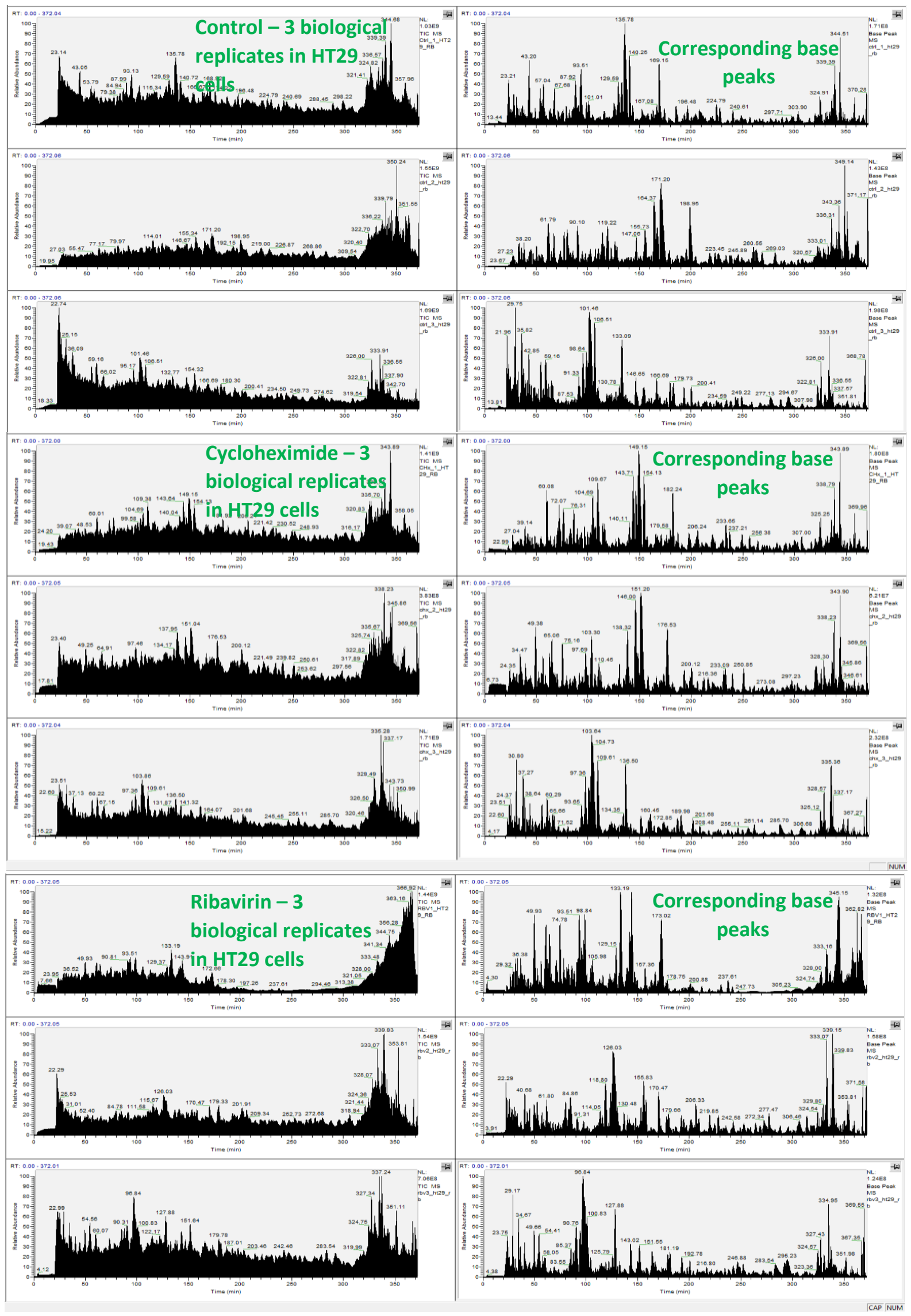

Figure 6-8 Relating to section 4.7.2, three biological replicates are displayed for the untreated controls, cycloheximide and ribavirin. Displayed in the left panels is the unfiltered LC-MS ${ }^{2}$ chromatogram, in the right panels the base peaks are isolated from the unfiltered data. 


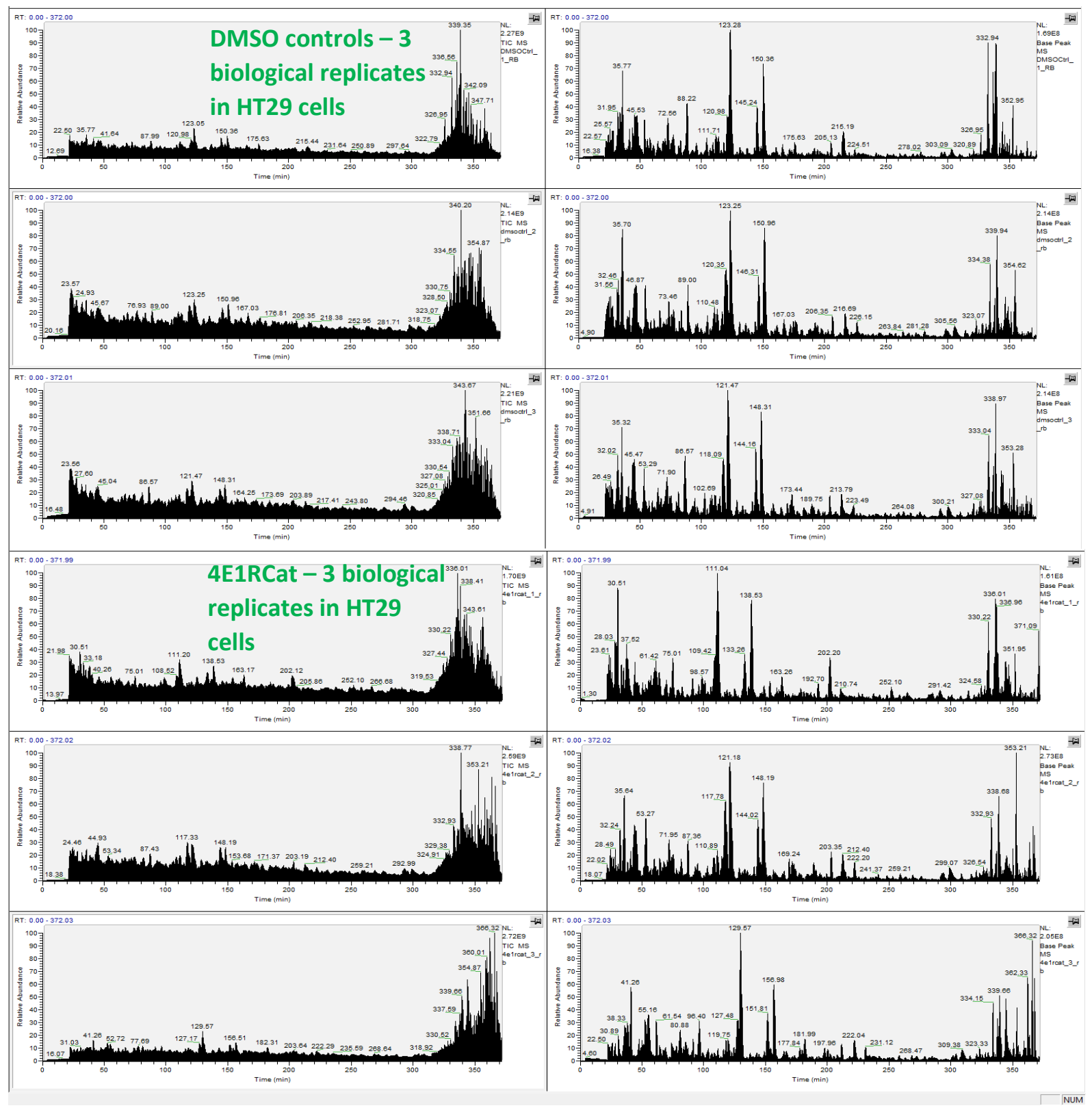

Figure 6-9 Relating to section 4.7.3, three biological replicates are displayed for 4E1RCat and the DMSO control. Ddisplayed in the left panels is the unfiltered LC-MS chromatogram, in the right panels the base peaks are isolated from the unfiltered data. 
Table 7 Proteins responding to treatment with cycloheximide.

\begin{tabular}{|c|c|c|c|c|c|}
\hline Identified Proteins & $\begin{array}{l}\text { T-Test } \\
\text { (p- } \\
\text { Value) }\end{array}$ & $\begin{array}{l}\text { Up or } \\
\text { Down } \\
\text { regulated }\end{array}$ & Identified Proteins & $\begin{array}{l}\text { T-Test } \\
\text { (p- } \\
\text { Value) }\end{array}$ & $\begin{array}{l}\text { Up or } \\
\text { Down } \\
\text { regulate } \\
\text { d }\end{array}$ \\
\hline $\begin{array}{l}\text { PSME2 protein OS=Homo } \\
\text { sapiens } G N=P S M E 2 \text { PE=2 } \\
S V=1\end{array}$ & 0.048 & Down & $\begin{array}{l}\text { Cluster of Sialic acid } \\
\text { synthase OS=Homo } \\
\text { sapiens GN=NANS PE=1 } \\
\text { SV=2 (SIAS_HUMAN) }\end{array}$ & 0.045 & Up \\
\hline $\begin{array}{l}\text { Cluster of cDNA } \\
\text { FL54047, highly similar } \\
\text { to Alpha-1 catenin } \\
\text { (Cadherin-associated } \\
\text { protein) OS=Homo } \\
\text { sapiens PE=2 SV=1 } \\
\text { (B4E2G8_HUMAN) }\end{array}$ & 0.048 & Down & $\begin{array}{l}\text { Testicular tissue protein } \\
\text { Li } 27 \text { OS=Homo sapiens } \\
\text { PE=2 SV=1 }\end{array}$ & 0.039 & Up \\
\hline $\begin{array}{l}\text { SUMO-conjugating } \\
\text { enzyme OS=Homo } \\
\text { sapiens } P E=2 \text { SV=1 }\end{array}$ & 0.047 & Down & $\begin{array}{l}\text { cDNA FL76092, highly } \\
\text { similar to Homo sapiens } \\
\text { 5'-nucleotidase, cytosolic } \\
\text { II-like } 1 \text { (NT5C2L1), mRNA } \\
\text { OS=Homo sapiens PE=2 } \\
\text { SV=1 }\end{array}$ & 0.034 & Up \\
\hline $\begin{array}{l}\text { Endoribonuclease LACTB2 } \\
\text { OS=Homo sapiens } \\
\text { GN=LACTB2 PE=1 SV=2 }\end{array}$ & 0.046 & Down & $\begin{array}{l}\text { Cluster of } 26 \mathrm{~S} \text { protease } \\
\text { regulatory subunit } 6 \mathrm{~A} \\
\text { OS=Homo sapiens } \\
\text { GN=PSMC3 } \mathrm{PE}=1 \mathrm{SV}=1 \\
\text { (E9PM69_HUMAN) }\end{array}$ & 0.033 & Up \\
\hline $\begin{array}{l}\text { Ubiquitin-conjugating } \\
\text { enzyme E2 K OS=Homo } \\
\text { sapiens } G N=U B E 2 K \text { PE=1 } \\
\text { SV=3 }\end{array}$ & 0.046 & Down & $\begin{array}{l}\text { Cluster of Epididymis } \\
\text { secretory protein Li } 21 \\
\text { OS=Homo sapiens } \\
\text { GN=HEL-S-21 PE=2 SV=1 } \\
\text { (V9HWG9_HUMAN) }\end{array}$ & 0.032 & Up \\
\hline $\begin{array}{l}\text { Cluster of Ubiquitin } \\
\text { thioesterase OS=Homo } \\
\text { sapiens PE=2 SV=1 } \\
\text { (B4DPD5_HUMAN) }\end{array}$ & 0.044 & Down & $\begin{array}{l}\text { Vacuolar protein sorting- } \\
\text { associated protein } 29 \\
\text { OS=Homo sapiens } \\
\text { GN=VPS29 PE=1 SV=1 }\end{array}$ & 0.03 & Up \\
\hline $\begin{array}{l}\text { MARCKS-related protein } \\
\text { OS=Homo sapiens } \\
\text { GN=MARCKSL1 PE=1 } \\
\text { SV=2 }\end{array}$ & 0.036 & Down & $\begin{array}{l}\text { 39S ribosomal protein } \\
\text { L39, mitochondrial } \\
\text { OS=Homo sapiens } \\
\text { GN=MRPL39 PE=1 SV=3 }\end{array}$ & 0.028 & Up \\
\hline $\begin{array}{l}\text { Cluster of cDNA } \\
\text { PSEC0016 fis, clone } \\
\text { NT2RM1001076, highly } \\
\text { similar to Procollagen- } \\
\text { lysine,2-oxoglutarate 5- } \\
\text { dioxygenase } 3 \text { (EC } \\
\text { 1.14.11.4) OS=Homo }\end{array}$ & 0.036 & Down & $\begin{array}{l}\text { Cluster of } \\
\text { Serine/threonine-protein } \\
\text { phosphatase PP1-alpha } \\
\text { catalytic subunit } \\
\text { OS=Homo sapiens } \\
\text { GN=PPP1CA PE=1 SV=1 } \\
\text { (PP1A_HUMAN) }\end{array}$ & 0.028 & Up \\
\hline
\end{tabular}




\begin{tabular}{|c|c|c|c|c|c|}
\hline $\begin{array}{l}\text { sapiens } P E=2 \mathrm{SV}=1 \\
\text { (B3KQQ3_HUMAN) }\end{array}$ & & & & & \\
\hline $\begin{array}{l}\text { Cluster of cDNA } \\
\text { FL55694, highly similar } \\
\text { to Dipeptidyl-peptidase } 1 \\
\text { (EC 3.4.14.1) OS=Homo } \\
\text { sapiens PE=2 SV=1 } \\
\text { (B4DJQ8_HUMAN) }\end{array}$ & 0.034 & Down & $\begin{array}{l}\text { RNA binding protein } \\
\text { (Autoantigenic, hnRNP- } \\
\text { associated with lethal } \\
\text { yellow) long isoform } \\
\text { variant (Fragment) } \\
\text { OS=Homo sapiens } \\
\text { GN=RALY PE=1 SV=1 }\end{array}$ & 0.027 & Up \\
\hline $\begin{array}{l}\text { Splicing factor 3B subunit } \\
3 \text { OS=Homo sapiens } \\
\text { GN=SF3B3 PE=1 SV=4 }\end{array}$ & 0.031 & Down & $\begin{array}{l}\text { Cluster of Small nuclear } \\
\text { ribonucleoprotein- } \\
\text { associated protein N } \\
\text { OS=Homo sapiens } \\
\text { GN=SNRPN PE=1 SV=1 } \\
\text { (RSMN_HUMAN) }\end{array}$ & 0.026 & Up \\
\hline $\begin{array}{l}\text { Synaptic vesicle } \\
\text { membrane protein VAT-1 } \\
\text { homolog OS=Homo } \\
\text { sapiens GN=VAT1 PE=1 } \\
\text { SV=2 }\end{array}$ & 0.031 & Down & $\begin{array}{l}\text { Cluster of Protein canopy } \\
\text { homolog } 2 \text { OS=Homo } \\
\text { sapiens GN=CNPY2 PE=1 } \\
\text { SV=1 (CNPY2_HUMAN) }\end{array}$ & 0.025 & Up \\
\hline $\begin{array}{l}\text { Cluster of Spermine } \\
\text { synthase OS=Homo } \\
\text { sapiens GN=SMS PE=1 } \\
\text { SV=2 (SPSY_HUMAN) }\end{array}$ & 0.03 & Down & $\begin{array}{l}\text { Serpin B6 OS=Homo } \\
\text { sapiens GN=SERPINB6 } \\
\mathrm{PE}=1 \mathrm{SV}=1\end{array}$ & 0.017 & Up \\
\hline $\begin{array}{l}\text { L-aminoadipate- } \\
\text { semialdehyde } \\
\text { dehydrogenase- } \\
\text { phosphopantetheinyl } \\
\text { transferase OS=Homo } \\
\text { sapiens GN=AASDHPPT } \\
\mathrm{PE}=1 \mathrm{SV}=2\end{array}$ & 0.026 & Down & $\begin{array}{l}\text { 40S ribosomal protein } \\
\text { S12 OS=Homo sapiens } \\
\text { GN=RPS12 PE=1 SV=3 }\end{array}$ & 0.017 & Up \\
\hline $\begin{array}{l}\text { Cluster of Proteasome } \\
\text { subunit alpha type } \\
\text { OS=Homo sapiens } \\
\text { GN=PSMA6 PE=1 SV=1 } \\
\text { (G3V295_HUMAN) }\end{array}$ & 0.026 & Down & $\begin{array}{l}\text { ATP synthase subunit } \\
\text { gamma } O S=H o m o \\
\text { sapiens } P E=2 S V=1\end{array}$ & 0.015 & Up \\
\hline $\begin{array}{l}\text { Cluster of DNA replication } \\
\text { licensing factor MCM4 } \\
\text { OS=Homo sapiens } \\
\text { GN=MCM4 PE=1 SV=5 } \\
\text { (MCM4_HUMAN) }\end{array}$ & 0.024 & Down & $\begin{array}{l}\text { cDNA FL39996 fis, clone } \\
\text { STOMA2002166, highly } \\
\text { similar to Splicing factor } \\
\text { 3B subunit } 4 \text { OS=Homo } \\
\text { sapiens PE=2 SV=1 }\end{array}$ & 0.015 & Up \\
\hline $\begin{array}{l}\text { Cluster of Epididymis } \\
\text { secretory protein Li } 71 \\
\text { OS=Homo sapiens } \\
\text { GN=HEL-S-71 PE=2 SV=1 } \\
\text { (V9HW41_HUMAN) }\end{array}$ & 0.022 & Down & $\begin{array}{l}\text { Stathmin OS=Homo } \\
\text { sapiens GN=STMN1 PE=2 } \\
\text { SV=1 }\end{array}$ & 0.014 & Up \\
\hline $\begin{array}{l}\text { Cluster of cDNA } \\
\text { FLJ76962, highly similar }\end{array}$ & 0.021 & Down & $\begin{array}{l}\text { Eukaryotic translation } \\
\text { initiation factor } 3 \text { subunit }\end{array}$ & 0.013 & Up \\
\hline
\end{tabular}




\begin{tabular}{|c|c|c|c|c|c|}
\hline $\begin{array}{l}\text { to Homo sapiens } \\
\text { nucleolar protein } 5 \mathrm{~A} \\
\text { ( } 56 \mathrm{kDa} \text { with KKE/D } \\
\text { repeat) (NOL5A), mRNA } \\
\text { OS=Homo sapiens PE=2 } \\
\text { SV=1 (A8K9K6_HUMAN) }\end{array}$ & & & $\begin{array}{l}\text { M OS=Homo sapiens } \\
G N=E I F 3 M \quad P E=1 S V=1\end{array}$ & & \\
\hline $\begin{array}{l}\text { Similar to NADH } \\
\text { dehydrogenase } \\
\text { (Ubiquinone) } 1 \text { alpha } \\
\text { subcomplex, } 9 \text { (39kD) } \\
\text { (Fragment) OS=Homo } \\
\text { sapiens PE=2 SV=1 }\end{array}$ & 0.02 & Down & $\begin{array}{l}\text { UPF0160 protein MYG1, } \\
\text { mitochondrial OS=Homo } \\
\text { sapiens } \mathrm{GN}=\mathrm{C} 12 \text { orf10 } \\
\mathrm{PE}=1 \mathrm{SV}=2\end{array}$ & 0.012 & Up \\
\hline $\begin{array}{l}\text { Peptidyl-prolyl cis-trans } \\
\text { isomerase NIMA- } \\
\text { interacting } 1 \text { OS=Homo } \\
\text { sapiens GN=PIN1 PE=1 } \\
\text { SV=1 }\end{array}$ & 0.016 & Down & $\begin{array}{l}\text { SF3A2 protein (Fragment) } \\
\text { OS=Homo sapiens } \\
\mathrm{GN}=\mathrm{SF} 3 \mathrm{~A} 2 \mathrm{PE}=2 \mathrm{SV}=1\end{array}$ & 0.012 & Up \\
\hline $\begin{array}{l}\text { Hsp70-binding protein } 1 \\
\text { OS=Homo sapiens } \\
\text { GN=HSPBP1 PE=1 SV=1 }\end{array}$ & 0.015 & Down & $\begin{array}{l}\text { Cluster of cDNA, } \\
\text { FL94136, highly similar } \\
\text { to Homo sapiens } \\
\text { synaptotagmin binding, } \\
\text { cytoplasmic RNA } \\
\text { interacting protein } \\
\text { (SYNCRIP), mRNA } \\
\text { OS=Homo sapiens PE=2 } \\
\text { SV=1 (B2R8Z8_HUMAN) }\end{array}$ & 0.011 & Up \\
\hline $\begin{array}{l}\text { Cluster of DDAH2 } \\
\text { OS=Homo sapiens } \\
\text { GN=HEL-S-277 PE=1 SV=1 } \\
\text { (V9HW53_HUMAN) }\end{array}$ & 0.015 & Down & $\begin{array}{l}\text { 60S ribosomal protein } \\
\text { L12 OS=Homo sapiens } \\
\text { GN=RPL12 PE=1 SV=1 }\end{array}$ & 0.0087 & Up \\
\hline $\begin{array}{l}\text { Cluster of Elongation } \\
\text { factor 1-alpha } 2 \\
\text { OS=Homo sapiens } \\
\text { GN=EEF1A2 PE=1 SV=1 } \\
\text { (EF1A2_HUMAN) }\end{array}$ & 0.015 & Down & $\begin{array}{l}\text { 7-alpha-hydroxycholest- } \\
\text { 4-en-3-one 12-alpha- } \\
\text { hydroxylase OS=Homo } \\
\text { sapiens GN=CYP8B1 PE=1 } \\
\text { SV=1 }\end{array}$ & 0.0087 & Up \\
\hline $\begin{array}{l}\text { E3 ubiquitin-protein } \\
\text { ligase } \mathrm{CHIP} \text { OS=Homo } \\
\text { sapiens GN=STUB1 PE=1 } \\
\text { SV=2 }\end{array}$ & 0.015 & Down & $\begin{array}{l}\text { Cluster of Testicular } \\
\text { tissue protein Li } 192 \\
\text { OS=Homo sapiens PE=2 } \\
\text { SV=1 } \\
\text { (AOA140VJW5_HUMAN) }\end{array}$ & 0.0063 & Up \\
\hline $\begin{array}{l}\text { Synaptogyrin-2 } \\
\text { (Fragment) OS=Homo } \\
\text { sapiens GN=SYNGR2 PE=1 } \\
\text { SV=1 }\end{array}$ & 0.014 & Down & $\begin{array}{l}\text { Cluster of Hepatoma- } \\
\text { derived growth factor } \\
\text { OS=Homo sapiens } \\
\text { GN=HDGF PE=1 SV=1 } \\
\text { (HDGF_HUMAN) }\end{array}$ & 0.0053 & Up \\
\hline $\begin{array}{l}\text { DNA replication licensing } \\
\text { factor } \mathrm{MCM} 6 \text { OS=Homo }\end{array}$ & 0.013 & Down & $\begin{array}{l}\text { Phosphoserine } \\
\text { phosphatase, isoform }\end{array}$ & 0.0052 & Up \\
\hline
\end{tabular}




\begin{tabular}{|c|c|c|c|c|c|}
\hline $\begin{array}{l}\text { sapiens } \mathrm{GN}=\mathrm{MCM} 6 \mathrm{PE}=1 \\
\mathrm{SV}=1\end{array}$ & & & $\begin{array}{l}\text { CRA_b OS=Homo sapiens } \\
\text { GN=PSPH PE=4 SV=1 }\end{array}$ & & \\
\hline $\begin{array}{l}\text { Cluster of Epididymis } \\
\text { luminal protein } 220 \\
\text { OS=Homo sapiens } \\
\text { GN=HEL-S-70 PE=2 SV=1 } \\
\text { (V9HW80_HUMAN) }\end{array}$ & 0.013 & Down & $\begin{array}{l}\text { Peroxiredoxin-5, } \\
\text { mitochondrial OS=Homo } \\
\text { sapiens GN=PRDX5 PE=1 } \\
\text { SV=4 }\end{array}$ & 0.0047 & Up \\
\hline $\begin{array}{l}\text { Proteasome subunit beta } \\
\text { type } O S=\text { Homo sapiens } \\
P E=2 S V=1\end{array}$ & 0.013 & Down & $\begin{array}{l}\text { Cluster of RNA-binding } \\
\text { motif protein, } X \\
\text { chromosome OS=Homo } \\
\text { sapiens GN=RBMX PE=1 } \\
\text { SV=3 (RBMX_HUMAN) }\end{array}$ & 0.0044 & Up \\
\hline $\begin{array}{l}\text { Splicing factor 3B subunit } \\
5 \text { OS=Homo sapiens } \\
\text { GN=SF3B5 PE=1 SV=1 }\end{array}$ & 0.01 & Down & $\begin{array}{l}\text { cDNA FL90381 fis, clone } \\
\text { NT2RP2005035, highly } \\
\text { similar to Calumenin } \\
\text { OS=Homo sapiens PE=2 } \\
\text { SV=1 }\end{array}$ & 0.0021 & Up \\
\hline $\begin{array}{l}\text { Cluster of Epiplakin } \\
\text { OS=Homo sapiens } \\
\text { GN=EPPK1 PE=1 SV=1 } \\
\text { (A0A087X1U6_HUMAN) }\end{array}$ & 0.01 & Down & $\begin{array}{l}\text { Asparagine--tRNA ligase, } \\
\text { cytoplasmic OS=Homo } \\
\text { sapiens GN=NARS PE=1 } \\
\text { SV=1 }\end{array}$ & 0.0011 & Up \\
\hline $\begin{array}{l}\text { UPF1 regulator of } \\
\text { nonsense transcripts } \\
\text { homolog (Yeast), isoform } \\
\text { CRA_b OS=Homo sapiens } \\
\text { GN=UPF1 PE=4 SV=1 }\end{array}$ & 0.0087 & Down & $\begin{array}{l}\text { Aspartate } \\
\text { aminotransferase } \\
\mathrm{OS}=\text { Homo sapiens } \mathrm{PE}=2 \\
\mathrm{SV}=1\end{array}$ & $\begin{array}{l}0.0008 \\
8\end{array}$ & Up \\
\hline $\begin{array}{l}\text { Cluster of Glutamate } \\
\text { dehydrogenase OS=Homo } \\
\text { sapiens PE=2 SV=1 } \\
\text { (B4DMF5_HUMAN) }\end{array}$ & 0.0083 & Down & $\begin{array}{l}\text { Glyoxylate } \\
\text { reductase/hydroxypyruva } \\
\text { te reductase OS=Homo } \\
\text { sapiens GN=GRHPR PE=1 } \\
\text { SV=1 }\end{array}$ & 0.0008 & Up \\
\hline $\begin{array}{l}\text { 40S ribosomal protein } \\
\text { S10 OS=Homo sapiens } \\
\text { GN=RPS10 PE=1 SV=1 }\end{array}$ & 0.0075 & Down & $\begin{array}{l}\text { Dynein heavy chain } 14 \text {, } \\
\text { axonemal OS=Homo } \\
\text { sapiens GN=DNAH14 } \\
\mathrm{PE}=2 \mathrm{SV}=3\end{array}$ & $\begin{array}{l}< \\
0.0001 \\
0\end{array}$ & Up \\
\hline $\begin{array}{l}\text { S- } \\
\text { (hydroxymethyl)glutathio } \\
\text { ne dehydrogenase } \\
\text { OS=Homo sapiens } \\
\text { GN=ADH5 PE=2 SV=1 }\end{array}$ & 0.0073 & Down & & & \\
\hline $\begin{array}{l}\text { Aminoacylase-1 } \\
\text { OS=Homo sapiens } \\
\text { GN=ACY1 PE=4 SV=1 }\end{array}$ & 0.0065 & Down & & & \\
\hline $\begin{array}{l}\text { Phosphomannomutase } \\
\text { OS=Homo sapiens } \\
\text { GN=PMM2 PE=1 SV=1 }\end{array}$ & 0.0059 & Down & & & \\
\hline
\end{tabular}




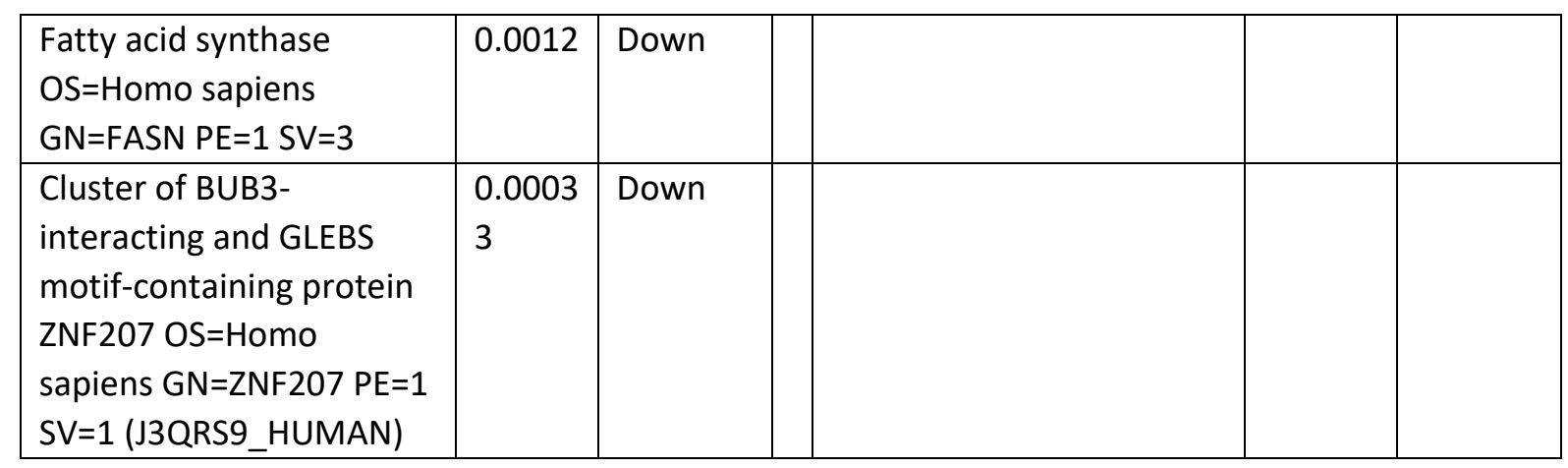


Table 8 Proteins responding to treatment with ribavirin.

\begin{tabular}{|c|c|c|c|c|c|}
\hline Identified Proteins & $\begin{array}{l}\text { T-Test } \\
\text { (p- } \\
\text { Value) }\end{array}$ & $\begin{array}{l}\text { Up or } \\
\text { Down } \\
\text { regulated }\end{array}$ & Identified Proteins & $\begin{array}{l}\text { T-Test } \\
\text { (p- } \\
\text { Value) }\end{array}$ & $\begin{array}{l}\text { Up or } \\
\text { Down } \\
\text { regulated }\end{array}$ \\
\hline $\begin{array}{l}\text { Cluster of Histone H2B } \\
\text { type 1-J OS=Homo } \\
\text { sapiens GN=HIST1H2BJ } \\
\text { PE=1 SV=3 } \\
\text { (H2B1J_HUMAN) }\end{array}$ & 0.0067 & Down & $\begin{array}{l}\text { Ribosomal L1 domain- } \\
\text { containing protein } 1 \\
\text { OS=Homo sapiens } \\
\text { GN=RSL1D1 PE=1 SV=3 }\end{array}$ & 0.0087 & Down \\
\hline $\begin{array}{l}\text { Cluster of Epididymis } \\
\text { luminal protein } 220 \\
\text { OS=Homo sapiens } \\
\text { GN=HEL-S-70 PE=2 SV=1 } \\
\text { (V9HW80_HUMAN) }\end{array}$ & 0.037 & Down & $\begin{array}{l}\text { cDNA FL56566, highly } \\
\text { similar to Small } \\
\text { glutamine-rich } \\
\text { tetratricopeptiderepeat } \\
\text {-containing protein A } \\
\text { OS=Homo sapiens PE=2 } \\
\text { SV=1 }\end{array}$ & 0.046 & Down \\
\hline $\begin{array}{l}\text { Cluster of Probable ATP- } \\
\text { dependent RNA helicase } \\
\text { DDX17 OS=Homo sapiens } \\
\text { GN=DDX17 PE=1 SV=1 } \\
\text { (A0A1W2PQ51_HUMAN) }\end{array}$ & 0.014 & Down & $\begin{array}{l}\text { Cluster of cDNA, } \\
\text { FL92825, highly similar } \\
\text { to Homo sapiens SAR1a } \\
\text { gene homolog } 1 \text { (S. } \\
\text { cerevisiae) (SARA1), } \\
\text { mRNA OS=Homo } \\
\text { sapiens PE=2 SV=1 } \\
\text { (B2R679_HUMAN) }\end{array}$ & 0.021 & Down \\
\hline $\begin{array}{l}\text { Cluster of RPS4X protein } \\
\text { (Fragment) OS=Homo } \\
\text { sapiens GN=RPS4X PE=2 } \\
\text { SV=2 (Q96IR1_HUMAN) }\end{array}$ & 0.019 & Down & $\begin{array}{l}\text { Cluster of NOP56 } \\
\text { protein (Fragment) } \\
\text { OS=Homo sapiens } \\
\text { GN=NOP56 PE=2 SV=1 } \\
\text { (AOPJ92_HUMAN) }\end{array}$ & 0.038 & Down \\
\hline $\begin{array}{l}\text { Cluster of Serine } \\
\text { hydroxymethyltransferas } \\
\text { e, mitochondrial } \\
\text { (Fragment) OS=Homo } \\
\text { sapiens GN=SHMT2 PE=1 } \\
\text { SV=1 (G3V4W5_HUMAN) }\end{array}$ & $\begin{array}{l}0.0008 \\
6\end{array}$ & Down & $\begin{array}{l}\text { Protein dpy-30 homolog } \\
\text { OS=Homo sapiens } \\
\text { GN=DPY30 PE=1 SV=1 }\end{array}$ & 0.017 & Down \\
\hline $\begin{array}{l}\text { Cluster of Ribosomal } \\
\text { protein L7, isoform } \\
\text { CRA_a OS=Homo sapiens } \\
\text { GN=RPL7 PE=4 SV=1 } \\
\text { (AOA024R814_HUMAN) }\end{array}$ & 0.029 & Down & $\begin{array}{l}\text { Hypoxanthine-guanine } \\
\text { phosphoribosyltransfer } \\
\text { ase OS=Homo sapiens } \\
\text { GN=HPRT1 PE=1 SV=2 }\end{array}$ & 0.027 & Down \\
\hline $\begin{array}{l}\text { Ribosomal protein L23, } \\
\text { isoform CRA_b OS=Homo } \\
\text { sapiens GN=RPL23 PE=3 } \\
\text { SV=1 }\end{array}$ & 0.0075 & Down & $\begin{array}{l}\text { DNA helicase OS=Homo } \\
\text { sapiens } \mathrm{GN}=\mathrm{MCM} 3 \\
\mathrm{PE}=2 \mathrm{SV}=1\end{array}$ & 0.0016 & Down \\
\hline $\begin{array}{l}40 \mathrm{~S} \text { ribosomal protein } \mathrm{S3} \\
\mathrm{OS}=\text { Homo sapiens } \\
\mathrm{GN}=\mathrm{RPS} 3 \mathrm{PE}=1 \mathrm{SV}=2\end{array}$ & 0.033 & Down & $\begin{array}{l}\text { ATP synthase subunit } \\
\text { delta, mitochondrial } \\
\text { OS=Homo sapiens } \\
\text { GN=ATP5D PE=1 SV=2 }\end{array}$ & 0.002 & Down \\
\hline
\end{tabular}




\begin{tabular}{|c|c|c|c|c|c|}
\hline $\begin{array}{l}\text { Cluster of Epididymis } \\
\text { secretory protein Li } 71 \\
\text { OS=Homo sapiens } \\
\text { GN=HEL-S-71 PE=2 SV=1 } \\
\text { (V9HW41_HUMAN) }\end{array}$ & 0.038 & Down & $\begin{array}{l}\text { BUB3-interacting and } \\
\text { GLEBS motif-containing } \\
\text { protein ZNF207 } \\
\text { OS=Homo sapiens } \\
\text { GN=ZNF207 PE=1 SV=1 }\end{array}$ & $\begin{array}{l}1.00 \mathrm{E}- \\
04\end{array}$ & Down \\
\hline $\begin{array}{l}\text { Cluster of Staphylococcal } \\
\text { nuclease domain- } \\
\text { containing protein } 1 \\
\text { OS=Homo sapiens } \\
\text { GN=SND1 PE=1 SV=1 } \\
\text { (SND1_HUMAN) }\end{array}$ & 0.009 & Down & $\begin{array}{l}\text { cDNA FLJ57877, highly } \\
\text { similar to Cleavage and } \\
\text { polyadenylation } \\
\text { specificity factor } 7 \\
\text { OS=Homo sapiens PE=2 } \\
\text { SV=1 }\end{array}$ & 0.046 & Down \\
\hline $\begin{array}{l}\text { 40S ribosomal protein } \\
\text { S3a OS=Homo sapiens } \\
\text { GN=RPS3A PE=2 SV=1 }\end{array}$ & 0.045 & Down & $\begin{array}{l}\text { Endothelial } \\
\text { differentiation-related } \\
\text { factor } 1 \text { OS=Homo } \\
\text { sapiens GN=EDF1 PE=1 } \\
\text { SV=1 }\end{array}$ & 0.029 & Down \\
\hline $\begin{array}{l}\text { Cluster of cDNA } \\
\text { FL59240, highly similar } \\
\text { to Far upstream element- } \\
\text { binding protein } 1 \\
\text { OS=Homo sapiens PE=2 } \\
\text { SV=1 (B4DWL1_HUMAN) }\end{array}$ & 0.014 & Down & $\begin{array}{l}\text { RNA-binding protein } 42 \\
\text { OS=Homo sapiens } \\
\text { GN=RBM42 PE=1 SV=1 }\end{array}$ & $\begin{array}{l}0.0006 \\
1\end{array}$ & Down \\
\hline $\begin{array}{l}\text { Cluster of Hydroxysteroid } \\
\text { (17-beta) dehydrogenase } \\
4 \text {, isoform CRA_b } \\
\text { (Fragment) OS=Homo } \\
\text { sapiens GN=HSD17B4 } \\
\text { PE=2 SV=1 } \\
\text { (AOAOS2Z4J1_HUMAN) }\end{array}$ & 0.048 & Down & & & \\
\hline $\begin{array}{l}\text { 40S ribosomal protein } \\
\text { S13 OS=Homo sapiens } \\
\text { GN=RPS13 PE=1 SV=2 }\end{array}$ & 0.043 & Down & $\begin{array}{l}\text { Cluster of Keratin, type I } \\
\text { cytoskeletal } 18 \\
\text { OS=Homo sapiens } \\
\text { GN=KRT18 PE=1 SV=2 } \\
\text { (K1C18_HUMAN) }\end{array}$ & 0.043 & Up \\
\hline $\begin{array}{l}\text { ATP synthase subunit O, } \\
\text { mitochondrial OS=Homo } \\
\text { sapiens GN=ATP5O PE=1 } \\
\text { SV=1 }\end{array}$ & 0.03 & Down & $\begin{array}{l}\text { Cluster of Actin, alpha } \\
\text { skeletal muscle } \\
\text { OS=Homo sapiens } \\
\text { GN=ACTA1 PE=1 SV=3 } \\
\text { (A6NL76_HUMAN) }\end{array}$ & 0.036 & Up \\
\hline $\begin{array}{l}\text { 60S ribosomal protein } \\
\text { L10a OS=Homo sapiens } \\
\text { GN=RPL10A PE=1 SV=2 }\end{array}$ & 0.0051 & Down & $\begin{array}{l}\text { Cluster of Annexin } \\
\text { OS=Homo sapiens } \\
\text { GN=ANXA2 PE=3 SV=1 } \\
\text { (A0A024R5Z7_HUMAN) }\end{array}$ & 0.04 & Up \\
\hline $\begin{array}{l}\text { 40S ribosomal protein } \\
\text { S30 OS=Homo sapiens } \\
G N=F A \cup P E=1 S V=1\end{array}$ & 0.015 & Down & $\begin{array}{l}\text { Heterogeneous nuclear } \\
\text { ribonucleoprotein } \mathrm{K} \\
\mathrm{OS}=\text { Homo sapiens } \\
\mathrm{GN}=\mathrm{HNRNPK} \mathrm{PE}=1 \mathrm{SV}=1\end{array}$ & 0.021 & Up \\
\hline
\end{tabular}




\begin{tabular}{|c|c|c|c|c|c|}
\hline $\begin{array}{l}\text { Cluster of Cyclin- } \\
\text { dependent kinase } 16 \\
\text { OS=Homo sapiens } \\
\text { GN=CDK16 PE=1 SV=1 } \\
\text { (CDK16_HUMAN) }\end{array}$ & 0.014 & Down & $\begin{array}{l}\text { Cluster of Annexin A1 } \\
\text { OS=Homo sapiens } \\
\text { GN=ANXA1 PE=1 SV=2 } \\
\text { (ANXA1_HUMAN) }\end{array}$ & $\begin{array}{l}0.0009 \\
5\end{array}$ & Up \\
\hline $\begin{array}{l}\text { 2'-5'-oligoadenylate } \\
\text { synthetase 3, 100kDa, } \\
\text { isoform CRA_a OS=Homo } \\
\text { sapiens GN=OAS3 PE=4 } \\
\text { SV=1 }\end{array}$ & 0.024 & Down & $\begin{array}{l}\text { Protein } \mathrm{S} 100-\mathrm{A6} \\
\mathrm{OS}=\text { Homo sapiens } \\
\mathrm{GN}=\mathrm{S} 100 \mathrm{~A} 6 \mathrm{PE}=1 \mathrm{SV}=1\end{array}$ & 0.049 & Up \\
\hline $\begin{array}{l}\text { Heterogeneous nuclear } \\
\text { ribonucleoprotein } \mathrm{R} \\
\text { OS=Homo sapiens } \\
\text { GN=HNRNPR PE=1 SV=1 }\end{array}$ & 0.043 & Down & $\begin{array}{l}\text { Cluster of Aspartate } \\
\text { aminotransferase } \\
\text { OS=Homo sapiens } \\
\text { GN=GOT2 PE=4 SV=1 } \\
\text { (AOA024R6W0_HUMAN } \\
\text { ) }\end{array}$ & 0.0081 & Up \\
\hline $\begin{array}{l}\text { Protein POF1B OS=Homo } \\
\text { sapiens GN=POF1B PE=1 } \\
\text { SV=3 }\end{array}$ & 0.012 & Down & $\begin{array}{l}\text { Cluster of MHC class I } \\
\text { antigen (Fragment) } \\
\text { OS=Homo sapiens } \\
\text { GN=HLA-A PE=3 SV=1 } \\
\text { (E5BBI6_HUMAN) }\end{array}$ & 0.042 & Up \\
\hline $\begin{array}{l}\text { Testicular tissue protein } \\
\text { Li } 75 \text { OS=Homo sapiens } \\
\text { PE=2 SV=1 }\end{array}$ & 0.033 & Down & $\begin{array}{l}\text { Cluster of Proteasome } \\
\text { subunit alpha type } \\
\text { OS=Homo sapiens } \\
\text { GN=PSMA6 PE=1 SV=1 } \\
\text { (G3V295_HUMAN) }\end{array}$ & 0.02 & Up \\
\hline $\begin{array}{l}\text { cDNA FL76387, highly } \\
\text { similar to Homo sapiens } \\
\text { splicing factor, } \\
\text { arginine/serine-rich } 9 \\
\text { (SFRS9), mRNA OS=Homo } \\
\text { sapiens PE=2 SV=1 }\end{array}$ & 0.041 & Down & $\begin{array}{l}\text { Cluster of ARP3 actin- } \\
\text { related protein } 3 \\
\text { homolog (Yeast), } \\
\text { isoform CRA_a } \\
\text { OS=Homo sapiens } \\
\text { GN=ACTR3 PE=3 SV=1 } \\
\text { (AOA024RAl1_HUMAN) }\end{array}$ & 0.011 & Up \\
\hline $\begin{array}{l}\text { Cluster of DNA helicase } \\
\text { OS=Homo sapiens PE=2 } \\
\text { SV=1 (B3KMXO_HUMAN) }\end{array}$ & 0.046 & Down & $\begin{array}{l}\text { Epididymis secretory } \\
\text { sperm binding protein Li } \\
\text { 47e OS=Homo sapiens } \\
\text { GN=HEL-S-47e PE=2 } \\
\text { SV=1 }\end{array}$ & 0.047 & Up \\
\hline $\begin{array}{l}\text { Epidermal growth factor } \\
\text { receptor kinase substrate } \\
\text { 8-like protein } 1 \text { OS=Homo } \\
\text { sapiens GN=EPS8L1 PE=1 } \\
\text { SV=1 }\end{array}$ & 0.046 & Down & $\begin{array}{l}\text { Prefoldin subunit } 2 \\
\text { OS=Homo sapiens } \\
\text { GN=PFDN2 PE=1 SV=1 }\end{array}$ & $\begin{array}{l}0.0001 \\
4\end{array}$ & Up \\
\hline $\begin{array}{l}\text { Small nuclear } \\
\text { ribonucleoprotein Sm D2 } \\
\text { OS=Homo sapiens } \\
\text { GN=SNRPD2 PE=1 SV=1 }\end{array}$ & 0.003 & Down & $\begin{array}{l}\text { Ubiquitin carboxyl- } \\
\text { terminal hydrolase } 14 \\
\text { OS=Homo sapiens } \\
\text { GN=USP14 PE=1 SV=2 }\end{array}$ & 0.022 & $U p$ \\
\hline
\end{tabular}




\begin{tabular}{|c|c|c|c|c|c|}
\hline $\begin{array}{l}\text { U1 small nuclear } \\
\text { ribonucleoprotein } 70 \mathrm{kDa} \\
\text { OS=Homo sapiens } \\
\text { GN=SNRNP70 PE=1 SV=2 }\end{array}$ & 0.022 & Down & $\begin{array}{l}\text { 14-3-3 protein sigma } \\
\text { OS=Homo sapiens } \\
\text { GN=SFN PE=1 SV=1 }\end{array}$ & 0.033 & Up \\
\hline $\begin{array}{l}\text { Cluster of Hydroxysteroid } \\
\text { dehydrogenase-like } \\
\text { protein } 2 \text { OS=Homo } \\
\text { sapiens GN=HSDL2 PE=1 } \\
\text { SV=1 (HSDL2_HUMAN) }\end{array}$ & 0.0084 & Down & $\begin{array}{l}\text { Cluster of Kallikrein I } \\
\text { OS=Homo sapiens } \\
\text { GN=KLNI PE=3 SV=1 } \\
\text { (AOA1R3UCE8_HUMAN) }\end{array}$ & 0.0086 & Up \\
\hline $\begin{array}{l}\text { ADP-sugar } \\
\text { pyrophosphatase } \\
\text { OS=Homo sapiens } \\
\text { GN=NUDT5 PE=1 SV=1 }\end{array}$ & 0.032 & Down & $\begin{array}{l}\text { Ubiquitin thioesterase } \\
\text { OS=Homo sapiens } \mathrm{PE}=2 \\
\mathrm{SV}=1\end{array}$ & 0.01 & Up \\
\hline $\begin{array}{l}\text { Cluster of } 40 \text { S ribosomal } \\
\text { protein } \mathrm{S} 6 \mathrm{OS}=\text { Homo } \\
\text { sapiens GN=RPS6 PE=2 } \\
\text { SV=1 (Q96DV6_HUMAN) }\end{array}$ & 0.032 & Down & $\begin{array}{l}\text { Cluster of Tight junction } \\
\text { protein ZO-1 (Fragment) } \\
\text { OS=Homo sapiens } \\
\text { GN=TJP1 PE=2 SV=1 } \\
\text { (A9CQZ8_HUMAN) }\end{array}$ & 0.02 & Up \\
\hline $\begin{array}{l}\text { Septin-9 OS=Homo } \\
\text { sapiens GN=SEPT9 PE=1 } \\
\text { SV=2 }\end{array}$ & 0.0077 & Down & $\begin{array}{l}\text { SUMO-activating } \\
\text { enzyme subunit } 2 \\
\text { OS=Homo sapiens } \\
\text { GN=UBA2 PE=1 SV=2 }\end{array}$ & 0.043 & Up \\
\hline $\begin{array}{l}\text { Cluster of FGFR2-BICC1 } \\
\text { fusion kinase protein } \\
\text { OS=Homo sapiens } \\
\text { GN=FGFR2-BICC1 PE=2 } \\
\text { SV=1 (V5YQU3_HUMAN) }\end{array}$ & 0.027 & Down & $\begin{array}{l}\text { ATP synthase subunit } \\
\text { gamma OS=Homo } \\
\text { sapiens } P E=2 S V=1\end{array}$ & $\begin{array}{l}0.0007 \\
2\end{array}$ & Up \\
\hline $\begin{array}{l}\text { Cluster of UPF1 regulator } \\
\text { of nonsense transcripts } \\
\text { homolog (Yeast), isoform } \\
\text { CRA_b OS=Homo sapiens } \\
\text { GN=UPF1 PE=4 SV=1 } \\
\text { (A0A024R7L5_HUMAN) }\end{array}$ & 0.04 & Down & $\begin{array}{l}\text { ATP-binding cassette } \\
\text { sub-family E member } 1 \\
\text { OS=Homo sapiens } \\
\text { GN=ABCE1 PE=1 SV=1 }\end{array}$ & 0.045 & Up \\
\hline $\begin{array}{l}\text { Cluster of } 40 \text { S ribosomal } \\
\text { protein } \mathrm{S} 10 \text { OS=Homo } \\
\text { sapiens GN=RPS10 PE=1 } \\
\text { SV=1 (RS10_HUMAN) }\end{array}$ & 0.038 & Down & $\begin{array}{l}\text { Nuclear transport factor } \\
2 \text { OS=Homo sapiens } \\
\text { GN=NUTF2 PE=1 SV=1 }\end{array}$ & 0.05 & Up \\
\hline $\begin{array}{l}\text { Ras-GTPase activating } \\
\text { protein SH3 domain- } \\
\text { binding protein 2, isoform } \\
\text { CRA_a OS=Homo sapiens } \\
\text { GN=G3BP2 PE=4 SV=1 }\end{array}$ & 0.0082 & Down & $\begin{array}{l}\text { Coatomer subunit delta } \\
\text { OS=Homo sapiens } \\
\text { GN=ARCN1 PE=2 SV=1 }\end{array}$ & 0.015 & Up \\
\hline $\begin{array}{l}\text { Phosphoserine } \\
\text { aminotransferase } \\
\text { OS=Homo sapiens } \mathrm{PE}=2 \\
\mathrm{SV}=1\end{array}$ & 0.0067 & Down & $\begin{array}{l}\text { Tubulin-folding cofactor } \\
B \text { (Fragment) OS=Homo } \\
\text { sapiens GN=TBCB PE=1 } \\
S V=8\end{array}$ & 0.015 & Up \\
\hline $\begin{array}{l}\text { Cluster of Pyrroline-5- } \\
\text { carboxylate reductase }\end{array}$ & 0.04 & Down & $\begin{array}{l}\text { SEC13-like } 1 \text { isoform b } \\
\text { variant (Fragment) }\end{array}$ & 0.0058 & Up \\
\hline
\end{tabular}




\begin{tabular}{|c|c|c|c|c|c|}
\hline $\begin{array}{l}\mathrm{OS}=\text { Homo sapiens } \mathrm{PE}=2 \\
\mathrm{SV}=1 \text { (E7D7X9_HUMAN) }\end{array}$ & & & $\begin{array}{l}\mathrm{OS}=\text { Homo sapiens } \mathrm{PE}=2 \\
\mathrm{SV}=1\end{array}$ & & \\
\hline $\begin{array}{l}\text { Endoribonuclease LACTB2 } \\
\text { OS=Homo sapiens } \\
\text { GN=LACTB2 PE=1 SV=2 }\end{array}$ & 0.018 & Down & $\begin{array}{l}\text { Apoptosis-associated } \\
\text { speck-like protein } \\
\text { containing a CARD } \\
\text { OS=Homo sapiens } \\
\text { GN=PYCARD PE=1 SV=2 }\end{array}$ & 0.04 & Up \\
\hline $\begin{array}{l}\text { Cluster of Acetyl-CoA } \\
\text { acetyltransferase, } \\
\text { cytosolic variant } \\
\text { (Fragment) OS=Homo } \\
\text { sapiens PE=2 SV=1 } \\
\text { (Q59GW6_HUMAN) }\end{array}$ & 0.0057 & Down & $\begin{array}{l}\text { Cluster of cDNA, } \\
\text { FL93976, highly similar } \\
\text { to Homo sapiens COP9 } \\
\text { homolog (COP9), mRNA } \\
\text { OS=Homo sapiens PE=2 } \\
\text { SV=1 (B2R8N1_HUMAN) }\end{array}$ & 0.046 & Up \\
\hline $\begin{array}{l}\text { Cluster of cDNA } \\
\text { FLJ75871, highly similar } \\
\text { to Homo sapiens staufen, } \\
\text { RNA binding protein } \\
\text { (STAU), transcript variant } \\
\text { T3, mRNA OS=Homo } \\
\text { sapiens PE=2 SV=1 } \\
\text { (A8K622_HUMAN) }\end{array}$ & 0.024 & Down & $\begin{array}{l}\text { Cluster of cDNA } \\
\text { FL55936, highly similar } \\
\text { to Polypyrimidine tract- } \\
\text { binding protein } 2 \\
\text { OS=Homo sapiens PE=2 } \\
\text { SV=1 (B4DSS8_HUMAN) }\end{array}$ & 0.049 & Up \\
\hline $\begin{array}{l}\text { Nucleolar RNA helicase } 2 \\
\text { OS=Homo sapiens } \\
\text { GN=DDX21 PE=1 SV=5 }\end{array}$ & 0.018 & Down & $\begin{array}{l}26 \mathrm{~S} \text { proteasome non- } \\
\text { ATPase regulatory } \\
\text { subunit } 14 \mathrm{OS}=\text { Homo } \\
\text { sapiens GN=PSMD14 } \\
\mathrm{PE}=1 \mathrm{SV}=1\end{array}$ & 0.0034 & Up \\
\hline $\begin{array}{l}\text { Cluster of Rho guanine } \\
\text { nucleotide exchange } \\
\text { factor } 1 \mathrm{OS}=\text { Homo } \\
\text { sapiens GN=ARHGEF1 } \\
\mathrm{PE}=1 \mathrm{SV}=2 \\
\text { (ARHG1_HUMAN) }\end{array}$ & 0.0012 & Down & $\begin{array}{l}\text { Putative } \\
\text { uncharacterized protein } \\
\text { DKFZp686B04128 } \\
\text { OS=Homo sapiens } \\
\text { GN=DKFZp686B04128 } \\
\text { PE=2 SV=1 }\end{array}$ & 0.0069 & Up \\
\hline
\end{tabular}


Table 9 Proteins responding to treatment with 4E1RCat

\begin{tabular}{|c|c|c|c|c|c|}
\hline Identified Proteins & $\begin{array}{l}\text { T-Test } \\
\text { (p- } \\
\text { Value) }\end{array}$ & $\begin{array}{l}\text { Up or } \\
\text { Down } \\
\text { regulated }\end{array}$ & Identified Proteins & $\begin{array}{l}\text { T-Test } \\
\text { (p- } \\
\text { Value) }\end{array}$ & $\begin{array}{l}\text { Up or } \\
\text { Down } \\
\text { regulated }\end{array}$ \\
\hline $\begin{array}{l}\text { Cluster of Keratin, type I } \\
\text { cytoskeletal } 18 \text { OS=Homo } \\
\text { sapiens GN=KRT18 PE=1 } \\
\text { SV=2 (K1C18_HUMAN) }\end{array}$ & 0.034 & Down & $\begin{array}{l}\text { 60S ribosomal } \\
\text { protein } \mathrm{L} 8 \\
\mathrm{OS}=\text { Homo sapiens } \\
\mathrm{GN}=\mathrm{RPL} 8 \mathrm{PE}=1 \\
\mathrm{SV}=2\end{array}$ & 0.034 & Up \\
\hline $\begin{array}{l}\text { Cluster of Epididymis } \\
\text { luminal protein } 33 \\
\text { OS=Homo sapiens } \\
\text { GN=HEL-S-72p PE=2 SV=1 } \\
\text { (V9HW22_HUMAN) }\end{array}$ & 0.047 & Down & $\begin{array}{l}\text { Cluster of Hypoxia } \\
\text { up-regulated } \\
\text { protein } 1 \mathrm{OS}=\text { Homo } \\
\text { sapiens GN=HYOU1 } \\
\mathrm{PE}=1 \mathrm{SV}=1 \\
\text { (HYOU1_HUMAN) }\end{array}$ & 0.025 & Up \\
\hline $\begin{array}{l}\text { Cluster of } \\
\text { Dehydrogenase/reductase } \\
\text { SDR family member 2, } \\
\text { mitochondrial OS=Homo } \\
\text { sapiens GN=DHRS2 PE=1 } \\
\text { SV=4 (DHRS2_HUMAN) }\end{array}$ & 0.024 & Down & $\begin{array}{l}\text { Cluster of ATP- } \\
\text { dependent RNA } \\
\text { helicase A } \\
\text { OS=Homo sapiens } \\
\text { GN=DHX9 PE=1 } \\
\text { SV=4 } \\
\text { (DHX9_HUMAN) }\end{array}$ & 0.05 & Up \\
\hline $\begin{array}{l}\text { Cluster of Titin OS=Homo } \\
\text { sapiens GN=TTN PE=1 } \\
\text { SV=1 } \\
\text { (AOAOAOMTS7_HUMAN) }\end{array}$ & 0.0014 & Down & $\begin{array}{l}\text { Peptidyl-prolyl cis- } \\
\text { trans isomerase } \\
\text { FKBP4 OS=Homo } \\
\text { sapiens GN=FKBP4 } \\
\text { PE=1 SV=3 }\end{array}$ & 0.012 & Up \\
\hline $\begin{array}{l}\text { Cluster of Adenylate } \\
\text { kinase } 2 \text {, mitochondrial } \\
\text { OS=Homo sapiens } \\
\text { GN=AK2 PE=1 SV=2 } \\
\text { (KAD2_HUMAN) }\end{array}$ & 0.014 & Down & $\begin{array}{l}\text { Cluster of } \\
\text { Bifunctional } \\
\text { glutamate/proline-- } \\
\text { tRNA ligase } \\
\text { OS=Homo sapiens } \\
\text { GN=EPRS PE=1 } \\
\text { SV=5 } \\
\text { (SYEP_HUMAN) }\end{array}$ & 0.027 & Up \\
\hline $\begin{array}{l}\text { Trifunctional enzyme } \\
\text { subunit beta, } \\
\text { mitochondrial OS=Homo } \\
\text { sapiens GN=HADHB PE=1 } \\
\text { SV=3 }\end{array}$ & 0.032 & Down & $\begin{array}{l}\text { Cluster of } \\
\text { Proteasome subunit } \\
\text { alpha type-1 } \\
\text { OS=Homo sapiens } \\
\text { GN=PSMA1 PE=1 } \\
\text { SV=1 } \\
\text { (PSA1_HUMAN) }\end{array}$ & 0.002 & Up \\
\hline $\begin{array}{l}\text { Cluster of Adenylyl } \\
\text { cyclase-associated protein } \\
\text { OS=Homo sapiens PE=2 } \\
\text { SV=1 (B2RDY9_HUMAN) }\end{array}$ & 0.022 & Down & $\begin{array}{l}\text { Cluster of } \\
\text { Sequestosome-1 } \\
\text { OS=Homo sapiens } \\
\text { GN=SQSTM1 PE=1 }\end{array}$ & 0.015 & Up \\
\hline
\end{tabular}




\begin{tabular}{|c|c|c|c|c|c|}
\hline & & & $\begin{array}{l}\text { SV=1 } \\
\text { (SQSTM_HUMAN) }\end{array}$ & & \\
\hline $\begin{array}{l}\text { Cluster of ATPase family } \\
\text { AAA domain-containing } \\
\text { protein } 3 A \text { (Fragment) } \\
\text { OS=Homo sapiens } \\
\text { GN=ATAD3A PE=1 SV=1 } \\
\text { (HOY2W2_HUMAN) }\end{array}$ & 0.043 & Down & $\begin{array}{l}\text { cDNA FL75881, } \\
\text { highly similar to } \\
\text { Homo sapiens } \\
\text { transferrin receptor } \\
\text { (p90, CD71) (TFRC), } \\
\text { mRNA OS=Homo } \\
\text { sapiens PE=2 SV=1 }\end{array}$ & 0.013 & Up \\
\hline $\begin{array}{l}\text { Lysozyme C OS=Homo } \\
\text { sapiens GN=LYZ PE=1 SV=1 }\end{array}$ & 0.022 & Down & $\begin{array}{l}\text { cDNA, FLI93269, } \\
\text { highly similar to } \\
\text { Homo sapiens } \\
\text { mitochondrial } \\
\text { ribosomal protein } \\
\text { L15 (MRPL15), } \\
\text { nuclear gene } \\
\text { encoding } \\
\text { mitochondrial } \\
\text { protein, mRNA } \\
\text { OS=Homo sapiens } \\
\text { PE=2 SV=1 }\end{array}$ & 0.044 & Up \\
\hline $\begin{array}{l}\text { Cluster of EH-domain } \\
\text { containing 4, isoform } \\
\text { CRA_a OS=Homo sapiens } \\
\text { GN=EHD4 PE=3 SV=1 } \\
\text { (A0A024R9N6_HUMAN) }\end{array}$ & 0.02 & Down & $\begin{array}{l}\text { 60S ribosomal } \\
\text { protein L34 } \\
\text { OS=Homo sapiens } \\
\text { GN=RPL34 PE=1 } \\
\text { SV=3 }\end{array}$ & 0.0095 & Up \\
\hline $\begin{array}{l}\text { Cytochrome c oxidase } \\
\text { subunit } 6 B 1 \text { OS=Homo } \\
\text { sapiens } G N=C O X 6 B 1 P E=1 \\
\text { SV=2 }\end{array}$ & 0.048 & Down & $\begin{array}{l}\text { Isocitrate } \\
\text { dehydrogenase } \\
{[\mathrm{NADP}] \mathrm{OS}=\text { Homo }} \\
\text { sapiens } \mathrm{PE}=2 \mathrm{SV}=1\end{array}$ & 0.023 & Up \\
\hline $\begin{array}{l}\text { Ubiquitin/ISG15- } \\
\text { conjugating enzyme E2 L6 } \\
\text { OS=Homo sapiens } \\
\text { GN=UBE2L6 PE=1 SV=4 }\end{array}$ & 0.019 & Down & $\begin{array}{l}\text { 60S ribosomal } \\
\text { protein L35 } \\
\text { OS=Homo sapiens } \\
\text { GN=RPL35 PE=1 } \\
\mathrm{SV}=2\end{array}$ & 0.015 & Up \\
\hline $\begin{array}{l}\text { Cysteine--tRNA ligase, } \\
\text { cytoplasmic OS=Homo } \\
\text { sapiens GN=CARS PE=1 } \\
\text { SV=1 }\end{array}$ & 0.048 & Down & $\begin{array}{l}\text { Epididymis } \\
\text { secretory protein Li } \\
102 \text { OS=Homo } \\
\text { sapiens GN=HEL-S- } \\
102 \text { PE=2 SV=1 }\end{array}$ & 0.0016 & Up \\
\hline $\begin{array}{l}\text { H/ACA ribonucleoprotein } \\
\text { complex subunit } 3 \\
\text { OS=Homo sapiens } \\
\mathrm{GN}=\mathrm{NOP} 10 \mathrm{PE}=1 \mathrm{SV}=1\end{array}$ & $\begin{array}{l}1.00 \\
E-04\end{array}$ & Down & $\begin{array}{l}\text { Aspartate } \\
\text { aminotransferase } \\
\mathrm{OS}=\text { Homo sapiens } \\
\mathrm{PE}=2 \mathrm{SV}=1\end{array}$ & 0.043 & Up \\
\hline $\begin{array}{l}\text { Cluster of PURA protein } \\
\text { (Fragment) OS=Homo } \\
\text { sapiens GN=PURA PE=2 } \\
\text { SV=1 (Q2NLC9_HUMAN) }\end{array}$ & 0.011 & Down & $\begin{array}{l}\text { Cluster of Heat } \\
\text { shock } 70 \mathrm{kDa} \\
\text { protein } 4 \mathrm{~L} \\
\text { OS=Homo sapiens }\end{array}$ & 0.014 & Up \\
\hline
\end{tabular}




\begin{tabular}{|c|c|c|c|c|c|}
\hline & & & $\begin{array}{l}\text { GN=HSPA4L PE=1 } \\
S V=1 \\
\text { (E9PDE8_HUMAN) }\end{array}$ & & \\
\hline $\begin{array}{l}\text { Mitochondrial } \\
\text { transcription factor } A \\
O S=\text { Homo sapiens } \mathrm{PE}=4 \\
\mathrm{SV}=1\end{array}$ & 0.021 & Down & $\begin{array}{l}\text { cDNA FL60607, } \\
\text { highly similar to } \\
\text { Acyl-protein } \\
\text { thioesterase } 1 \text { (EC } \\
\text { 3.1.2.-) OS=Homo } \\
\text { sapiens PE=2 SV=1 }\end{array}$ & 0.037 & Up \\
\hline $\begin{array}{l}\text { DNA helicase } \mathrm{OS}=\mathrm{Homo} \\
\text { sapiens } \mathrm{GN}=\mathrm{MCM} 3 \mathrm{PE}=2 \\
\mathrm{SV}=1\end{array}$ & 0.005 & Down & $\begin{array}{l}\text { Glutamate-- } \\
\text { cysteine ligase } \\
\text { regulatory subunit } \\
\text { OS=Homo sapiens } \\
\mathrm{GN}=\mathrm{GCLM} \mathrm{PE}=1 \\
\mathrm{SV}=1\end{array}$ & 0.042 & Up \\
\hline \multirow[t]{2}{*}{$\begin{array}{l}\text { Isochorismatase domain- } \\
\text { containing protein } 2 \\
\mathrm{OS}=\text { Homo sapiens } \\
\mathrm{GN}=\mathrm{ISOC} 2 \mathrm{PE}=1 \mathrm{SV}=1\end{array}$} & 0.024 & Down & $\begin{array}{l}\text { Acyl-protein } \\
\text { thioesterase } 2 \\
\text { OS=Homo sapiens } \\
\text { GN=LYPLA2 PE=1 } \\
\text { SV=1 }\end{array}$ & $\begin{array}{l}0.0007 \\
3\end{array}$ & Up \\
\hline & & & $\begin{array}{l}\text { Cluster of Heme } \\
\text { oxygenase } 2 \\
\text { (Fragment) } \\
\text { OS=Homo sapiens } \\
\text { GN=HMOX2 PE=1 } \\
\text { SV=1 } \\
\text { (I3L159_HUMAN) }\end{array}$ & 0.035 & Up \\
\hline $\begin{array}{l}\text { Cluster of 60S ribosomal } \\
\text { protein } L 3 \text { OS=Homo } \\
\text { sapiens GN=RPL3 PE=1 } \\
S V=2 \text { (RL3_HUMAN) }\end{array}$ & 0.012 & Up & $\begin{array}{l}\text { Cluster of cDNA } \\
\text { FL54671, highly } \\
\text { similar to Calcium- } \\
\text { binding } \\
\text { mitochondrial } \\
\text { carrier protein } \\
\text { Aralar2 OS=Homo } \\
\text { sapiens PE=2 SV=1 } \\
\text { (B7Z2E2_HUMAN) }\end{array}$ & 0.033 & Up \\
\hline $\begin{array}{l}\text { Cluster of Ribosomal } \\
\text { protein L10 isoform A } \\
\text { (Fragment) OS=Homo } \\
\text { sapiens GN=RPL10 PE=2 } \\
\text { SV=1 (X5D2T3_HUMAN) }\end{array}$ & 0.0034 & Up & $\begin{array}{l}\text { Bifunctional } \\
\text { coenzyme } A \\
\text { synthase OS=Homo } \\
\text { sapiens GN=COASY } \\
P E=1 \text { SV=4 }\end{array}$ & 0.0063 & $U p$ \\
\hline $\begin{array}{l}\text { Cluster of 60S ribosomal } \\
\text { protein } L 7 \text { a OS=Homo } \\
\text { sapiens GN=RPL7A PE=1 } \\
\text { SV=2 (RL7A_HUMAN) }\end{array}$ & 0.046 & Up & $\begin{array}{l}\text { Sulfiredoxin-1 } \\
\text { OS=Homo sapiens } \\
\text { GN=SRXN1 PE=1 } \\
\text { SV=2 }\end{array}$ & 0.018 & Up \\
\hline $\begin{array}{l}\text { Cluster of Thioredoxin } \\
\text { reductase } 1 \text {, cytoplasmic } \\
\text { OS=Homo sapiens }\end{array}$ & 0.041 & Up & $\begin{array}{l}\text { Nuclear protein } \\
\text { localization } 4 \\
\text { homolog }(\mathrm{S} .\end{array}$ & 0.044 & Up \\
\hline
\end{tabular}




\begin{tabular}{|c|c|c|c|c|c|}
\hline $\begin{array}{l}\mathrm{GN}=\text { TXNRD1 PE=1 SV=3 } \\
\text { (TRXR1_HUMAN) }\end{array}$ & & & $\begin{array}{l}\text { cerevisiae), isoform } \\
\text { CRA_a OS=Homo } \\
\text { sapiens } \\
\text { GN=NPLOC4 PE=4 } \\
\text { SV=1 }\end{array}$ & & \\
\hline $\begin{array}{l}\text { 60S ribosomal protein } \\
\text { L18a OS=Homo sapiens } \\
\text { GN=RPL18A PE=1 SV=2 }\end{array}$ & 0.0082 & Up & $\begin{array}{l}\text { Translocon- } \\
\text { associated protein } \\
\text { subunit delta } \\
\text { OS=Homo sapiens } \\
\text { GN=SSR4 PE=1 } \\
\text { SV=1 }\end{array}$ & 0.031 & Up \\
\hline
\end{tabular}

Table 10 Proteins responding to treatment with DMSO

\begin{tabular}{|c|c|c|c|c|c|}
\hline Identified Proteins & $\begin{array}{l}\text { T-Test } \\
\text { (p- } \\
\text { Value) }\end{array}$ & $\begin{array}{l}\text { Up or } \\
\text { Down } \\
\text { regulate } \\
\text { d }\end{array}$ & Identified Proteins & $\begin{array}{l}\text { T-Test } \\
\text { (p- } \\
\text { Value) }\end{array}$ & $\begin{array}{l}\text { Up or } \\
\text { Down } \\
\text { regulate } \\
\text { d }\end{array}$ \\
\hline $\begin{array}{l}\text { Cluster of Epididymis } \\
\text { luminal protein } 33 \\
\text { OS=Homo sapiens } \\
\text { GN=HEL-S-72p PE=2 } \\
\text { SV=1 } \\
\text { (V9HW22_HUMAN) }\end{array}$ & $\begin{array}{l}0.0006 \\
8\end{array}$ & Down & $\begin{array}{l}\text { Cluster of D- } \\
\text { dopachrome } \\
\text { tautomerase OS=Homo } \\
\text { sapiens GN=DDT PE=2 } \\
\text { SV=1 (Q53Y51_HUMAN) }\end{array}$ & 0.042 & Down \\
\hline $\begin{array}{l}\text { Cluster of Filamin-A } \\
\text { OS=Homo sapiens } \\
\text { GN=FLNA PE=1 SV=4 } \\
\text { (FLNA_HUMAN) }\end{array}$ & $\begin{array}{l}0.0001 \\
3\end{array}$ & Down & $\begin{array}{l}\text { Cluster of Alpha-enolase } \\
\text { OS=Homo sapiens } \\
\text { GN=ENO1 PE=1 SV=2 } \\
\text { (ENOA_HUMAN) }\end{array}$ & 0.043 & Down \\
\hline $\begin{array}{l}\text { Cluster of Polyadenylate- } \\
\text { binding protein } \\
\text { OS=Homo sapiens PE=2 } \\
\text { SV=1 (B4DQXO_HUMAN) }\end{array}$ & $\begin{array}{l}2.00 \mathrm{E}- \\
04\end{array}$ & Down & $\begin{array}{l}\text { Cluster of Eukaryotic } \\
\text { translation initiation } \\
\text { factor } 5 \text { A (Fragment) } \\
\text { OS=Homo sapiens } \\
\text { GN=EIF5A PE=1 SV=8 } \\
\text { (I3L397_HUMAN) }\end{array}$ & 0.043 & Down \\
\hline $\begin{array}{l}\text { Cluster of } \\
\text { Heterogeneous nuclear } \\
\text { ribonucleoprotein } \mathrm{K} \text {, } \\
\text { isoform CRA_d } \\
\text { OS=Homo sapiens } \\
\text { GN=HNRPK PE=4 SV=1 } \\
\text { (AOA024R228_HUMAN) }\end{array}$ & $\begin{array}{l}0.0003 \\
4\end{array}$ & Down & $\begin{array}{l}\text { Cluster of } \\
\text { Heterogeneous nuclear } \\
\text { ribonucleoprotein } \mathrm{H} 2 \\
\mathrm{OS}=\text { Homo sapiens } \\
\mathrm{GN}=\mathrm{HNRNPH} 2 \mathrm{PE}=1 \\
\mathrm{SV}=1 \text { (HNRH2_HUMAN) }\end{array}$ & 0.043 & Down \\
\hline $\begin{array}{l}\text { Cluster of } 60 \mathrm{~S} \text { acidic } \\
\text { ribosomal protein } \mathrm{P} 2 \\
\text { OS=Homo sapiens } \\
\text { GN=RPLP2 PE=1 SV=1 } \\
\text { (RLA2_HUMAN) }\end{array}$ & $\begin{array}{l}0.0006 \\
6\end{array}$ & Down & $\begin{array}{l}\text { Protein DJ-1 OS=Homo } \\
\text { sapiens GN=PARK7 PE=1 } \\
\text { SV=1 }\end{array}$ & 0.043 & Down \\
\hline $\begin{array}{l}\text { Cluster of T-complex } \\
\text { protein } 1 \text { subunit zeta-2 }\end{array}$ & 0.0011 & Down & $\begin{array}{l}\text { Splicing factor } 3 \mathrm{~A} \\
\text { subunit } 1 \mathrm{OS}=\text { Homo }\end{array}$ & 0.043 & Down \\
\hline
\end{tabular}




\begin{tabular}{|c|c|c|c|c|c|}
\hline $\begin{array}{l}\text { OS=Homo sapiens } \\
\text { GN=CCT6B PE=1 SV=5 } \\
\text { (TCPW_HUMAN) }\end{array}$ & & & $\begin{array}{l}\text { sapiens GN=SF3A1 PE=1 } \\
\text { SV=1 }\end{array}$ & & \\
\hline $\begin{array}{l}\text { Thioredoxin OS=Homo } \\
\text { sapiens GN=TXN PE=2 } \\
S V=1\end{array}$ & $\begin{array}{l}0.0004 \\
6\end{array}$ & Down & $\begin{array}{l}\text { Cluster of SWI/SNF } \\
\text { related, matrix } \\
\text { associated, actin } \\
\text { dependent regulator of } \\
\text { chromatin, subfamily e, } \\
\text { member } 1 \text {, isoform } \\
\text { CRA_a OS=Homo } \\
\text { sapiens GN=SMARCE1 } \\
\text { PE=4 SV=1 } \\
\text { (A0A024R1S7_HUMAN) }\end{array}$ & 0.043 & Down \\
\hline $\begin{array}{l}\text { Protein } \mathrm{S} 100 \mathrm{OS}=\text { Homo } \\
\text { sapiens } \mathrm{GN}=\mathrm{HEL}-\mathrm{S}-43 \\
\mathrm{PE}=2 \mathrm{SV}=1\end{array}$ & $\begin{array}{l}0.0005 \\
8\end{array}$ & Down & $\begin{array}{l}\text { cDNA FL37476 fis, clone } \\
\text { BRAWH2012827, highly } \\
\text { similar to Homo sapiens } \\
\text { BH3 interacting domain } \\
\text { death agonist (BID), } \\
\text { transcript variant 1, } \\
\text { mRNA OS=Homo } \\
\text { sapiens PE=2 SV=1 }\end{array}$ & 0.044 & Down \\
\hline $\begin{array}{l}\text { Cluster of cDNA } \\
\text { FL75422, highly similar } \\
\text { to Homo sapiens capping } \\
\text { protein (actin filament) } \\
\text { muscle Z-line, alpha 1, } \\
\text { mRNA OS=Homo sapiens } \\
\text { PE=2 SV=1 } \\
\text { (A8KOT9_HUMAN) }\end{array}$ & 0.0011 & Down & $\begin{array}{l}\text { Methylosome subunit } \\
\text { pICln OS=Homo sapiens } \\
\text { GN=CLNS1A PE=1 SV=1 }\end{array}$ & 0.044 & Down \\
\hline $\begin{array}{l}\text { Cluster of Nucleosome } \\
\text { assembly protein 1-like } 1 \\
\text { (Fragment) OS=Homo } \\
\text { sapiens GN=NAP1L1 } \\
\text { PE=1 SV=1 } \\
\text { (HOYHC3_HUMAN) }\end{array}$ & $\begin{array}{l}0.0007 \\
9\end{array}$ & Down & $\begin{array}{l}\text { Pyridoxine 5'-phosphate } \\
\text { oxidase variant } \\
\text { (Fragment) OS=Homo } \\
\text { sapiens PE=2 SV=1 }\end{array}$ & 0.044 & Down \\
\hline $\begin{array}{l}\text { Cluster of Cold shock } \\
\text { domain containing E1, } \\
\text { RNA-binding, isoform } \\
\text { CRA_a OS=Homo sapiens } \\
\text { GN=CSDE1 PE=4 SV=1 } \\
\text { (AOA024ROE2_HUMAN) }\end{array}$ & $\begin{array}{l}0.0008 \\
4\end{array}$ & Down & $\begin{array}{l}\text { Cluster of Ribonuclease } \\
\text { T2 OS=Homo sapiens } \\
\text { GN=RNASET2 PE=1 SV=1 } \\
\text { (AOA087WZM2_HUMAN } \\
\text { ) }\end{array}$ & 0.045 & Down \\
\hline $\begin{array}{l}\text { Protein POF1B OS=Homo } \\
\text { sapiens GN=POF1B PE=1 } \\
S V=3\end{array}$ & $\begin{array}{l}0.0004 \\
9\end{array}$ & Down & $\begin{array}{l}\text { Cluster of UPF1 } \\
\text { regulator of nonsense } \\
\text { transcripts homolog } \\
\text { (Yeast), isoform CRA_b } \\
\text { OS=Homo sapiens } \\
\text { GN=UPF1 PE=4 SV=1 } \\
\text { (AOA024R7L5_HUMAN) }\end{array}$ & 0.045 & Down \\
\hline
\end{tabular}




\begin{tabular}{|c|c|c|c|c|c|}
\hline $\begin{array}{l}\text { Cluster of Chromobox } \\
\text { protein homolog } 3 \\
\text { OS=Homo sapiens } \\
\text { GN=CBX3 PE=1 SV=4 } \\
\text { (CBX3_HUMAN) }\end{array}$ & $\begin{array}{l}0.0004 \\
2\end{array}$ & Down & $\begin{array}{l}\text { Chloride intracellular } \\
\text { channel protein } \\
\text { OS=Homo sapiens } P E=3 \\
S V=1\end{array}$ & 0.046 & Down \\
\hline $\begin{array}{l}\text { Catechol O- } \\
\text { methyltransferase } \\
\text { OS=Homo sapiens } \\
\text { GN=COMT PE=1 SV=2 }\end{array}$ & $\begin{array}{l}0.0008 \\
4\end{array}$ & Down & $\begin{array}{l}\text { Coactosin-like protein } \\
\text { OS=Homo sapiens } \\
G N=\text { COTL1 PE=1 SV=3 }\end{array}$ & 0.046 & Down \\
\hline $\begin{array}{l}\text { GRB2 protein (Fragment) } \\
\text { OS=Homo sapiens } \\
\mathrm{GN}=\mathrm{GRB} 2 \mathrm{PE}=2 \mathrm{SV}=1\end{array}$ & 0.0011 & Down & $\begin{array}{l}\text { Apoptosis inhibitor } 5 \\
\text { OS=Homo sapiens } \\
\text { GN=API5 PE=1 SV=3 }\end{array}$ & 0.046 & Down \\
\hline $\begin{array}{l}\text { Cluster of PDZ and LIM } \\
\text { domain protein } 5 \\
\text { OS=Homo sapiens } \\
\text { GN=PDLIM5 PE=1 SV=5 } \\
\text { (PDLI5_HUMAN) }\end{array}$ & $\begin{array}{l}0.0008 \\
1\end{array}$ & Down & $\begin{array}{l}\text { Eukaryotic translation } \\
\text { elongation factor } 1 \text { beta } \\
\text { 2, isoform CRA_a } \\
\text { OS=Homo sapiens } \\
\text { GN=EEF1B2 PE=3 SV=1 }\end{array}$ & 0.047 & Down \\
\hline $\begin{array}{l}\text { Cluster of Testicular } \\
\text { secretory protein Li } 8 \\
\text { OS=Homo sapiens } \mathrm{PE}=2 \\
\text { SV=1 } \\
\text { (AOA140VK08_HUMAN) }\end{array}$ & $\begin{array}{l}0.0004 \\
1\end{array}$ & Down & $\begin{array}{l}\text { Casein kinase II alpha } 1 \\
\text { subunit isoform a } \\
\text { (Fragment) OS=Homo } \\
\text { sapiens GN=CSNK2A1 } \\
\text { PE=2 SV=1 }\end{array}$ & 0.047 & Down \\
\hline $\begin{array}{l}\text { Protein S100-P } \\
\text { OS=Homo sapiens } \\
\text { GN=S100P PE=1 SV=2 }\end{array}$ & $\begin{array}{l}1.00 \mathrm{E}- \\
04\end{array}$ & Down & $\begin{array}{l}\text { Proteasome subunit } \\
\text { alpha type-3 OS=Homo } \\
\text { sapiens GN=PSMA3 } \\
P E=1 S V=2\end{array}$ & 0.048 & Down \\
\hline $\begin{array}{l}\text { Cluster of cDNA } \\
\text { FL56531, highly similar } \\
\text { to UV excision repair } \\
\text { protein RAD23 homolog } \\
\text { B OS=Homo sapiens } \\
\text { PE=2 SV=1 } \\
\text { (B4DEA3_HUMAN) }\end{array}$ & $\begin{array}{l}0.0006 \\
8\end{array}$ & Down & $\begin{array}{l}\text { Cluster of Proteasome } \\
\text { subunit beta type } \\
\text { (Fragment) OS=Homo } \\
\text { sapiens PE=2 SV=1 } \\
\text { (Q53FT8_HUMAN) }\end{array}$ & 0.048 & Down \\
\hline $\begin{array}{l}\text { Proteasome subunit beta } \\
\text { type-6 OS=Homo sapiens } \\
\text { GN=PSMB6 PE=1 SV=4 }\end{array}$ & $\begin{array}{l}0.0006 \\
5\end{array}$ & Down & $\begin{array}{l}\text { Cluster of ATP synthase } \\
\text { subunit beta (Fragment) } \\
\text { OS=Homo sapiens } \\
\text { GN=ATP5B PE=2 SV=1 } \\
\text { (Q0QEN7_HUMAN) }\end{array}$ & 0.049 & Down \\
\hline $\begin{array}{l}\text { Cluster of Band 4.1-like } \\
\text { protein } 1 \text { OS=Homo } \\
\text { sapiens GN=EPB41L1 } \\
\text { PE=1 SV=2 } \\
\text { (E41L1_HUMAN) }\end{array}$ & 0.0011 & Down & $\begin{array}{l}\text { Cluster of Septin-9 } \\
\text { OS=Homo sapiens } \\
\text { GN=SEPT9 PE=1 SV=2 } \\
\text { (SEPT9_HUMAN) }\end{array}$ & 0.049 & Down \\
\hline $\begin{array}{l}\text { Clathrin interactor } 1 \\
\text { isoform } 2 \text { (Fragment) } \\
\text { OS=Homo sapiens } \\
\text { GN=CLINT1 PE=2 SV=1 }\end{array}$ & $\begin{array}{l}0.0006 \\
7\end{array}$ & Down & $\begin{array}{l}\text { Cluster of } 26 \mathrm{~S} \\
\text { proteasome regulatory } \\
\text { subunit } 8 \mathrm{OS}=\text { Homo } \\
\text { sapiens GN=PSMC5 }\end{array}$ & 0.049 & Down \\
\hline
\end{tabular}




\begin{tabular}{|c|c|c|c|c|c|}
\hline & & & $\begin{array}{l}\mathrm{PE}=1 \mathrm{SV}=1 \\
\text { (PRS8_HUMAN) }\end{array}$ & & \\
\hline $\begin{array}{l}\text { Aminoacylase-1 } \\
\text { OS=Homo sapiens } \\
\text { GN=ACY1 PE=4 SV=1 }\end{array}$ & $\begin{array}{l}0.0003 \\
1\end{array}$ & Down & $\begin{array}{l}\text { Cluster of Histone H4 } \\
\text { OS=Homo sapiens } \\
\text { GN=HIST1H4A PE=1 } \\
\text { SV=2 (H4_HUMAN) }\end{array}$ & $\begin{array}{l}0.0009 \\
9\end{array}$ & Up \\
\hline $\begin{array}{l}\text { ATP synthase subunit } \\
\text { delta, mitochondrial } \\
\text { OS=Homo sapiens } \\
\text { GN=ATP5D PE=1 SV=2 }\end{array}$ & $\begin{array}{l}1.00 \mathrm{E}- \\
04\end{array}$ & Down & $\begin{array}{l}\text { Cluster of Thioredoxin } \\
\text { reductase } 1 \text {, cytoplasmic } \\
\text { OS=Homo sapiens } \\
\text { GN=TXNRD1 PE=1 SV=3 } \\
\text { (TRXR1_HUMAN) }\end{array}$ & $\begin{array}{l}0.0005 \\
9\end{array}$ & Up \\
\hline $\begin{array}{l}\text { BJ-HCC-24 tumor antigen } \\
\text { OS=Homo sapiens } \mathrm{PE}=2 \\
\mathrm{SV}=1\end{array}$ & $\begin{array}{l}0.0003 \\
5\end{array}$ & Down & $\begin{array}{l}\text { Cluster of 4F2 cell- } \\
\text { surface antigen heavy } \\
\text { chain OS=Homo sapiens } \\
\text { GN=SLC3A2 PE=1 SV=3 } \\
\text { (4F2_HUMAN) }\end{array}$ & 0.001 & Up \\
\hline $\begin{array}{l}\text { Cluster of Myosin light } \\
\text { chain 6B OS=Homo } \\
\text { sapiens GN=MYL6B PE=1 } \\
\text { SV=1 (F8W115_HUMAN) }\end{array}$ & 0.0013 & Down & $\begin{array}{l}\text { Histone H1.5 OS=Homo } \\
\text { sapiens GN=HIST1H1B } \\
\mathrm{PE}=1 \mathrm{SV}=3\end{array}$ & $\begin{array}{l}6.00 \mathrm{E}- \\
04\end{array}$ & Up \\
\hline $\begin{array}{l}\text { Cluster of Ras-GTPase } \\
\text { activating protein SH3 } \\
\text { domain-binding protein } \\
\text { 2, isoform CRA_a } \\
\text { OS=Homo sapiens } \\
\text { GN=G3BP2 PE=4 SV=1 } \\
\text { (AOA024RDE5_HUMAN) }\end{array}$ & 0.0013 & Down & $\begin{array}{l}\text { High mobility group } \\
\text { protein } B 2 \text { OS=Homo } \\
\text { sapiens GN=HMGB2 } \\
P E=1 S V=2\end{array}$ & $\begin{array}{l}0.0006 \\
4\end{array}$ & Up \\
\hline $\begin{array}{l}\text { Cluster of Tropomyosin } \\
\text { alpha-3 chain OS=Homo } \\
\text { sapiens GN=TPM3 PE=1 } \\
\text { SV=2 (TPM3_HUMAN) }\end{array}$ & 0.0014 & Down & $\begin{array}{l}\text { Cluster of } 40 \text { S ribosomal } \\
\text { protein } \mathrm{S} 10 \mathrm{OS}=\text { Homo } \\
\text { sapiens GN=RPS10 PE=1 } \\
\text { SV=1 (RS10_HUMAN) }\end{array}$ & $\begin{array}{l}0.0001 \\
7\end{array}$ & Up \\
\hline $\begin{array}{l}\text { Inorganic } \\
\text { pyrophosphatase } \\
\text { OS=Homo sapiens } \\
\text { GN=PPA1 PE=1 SV=2 }\end{array}$ & 0.0016 & Down & $\begin{array}{l}\text { Cluster of Voltage- } \\
\text { dependent anion } \\
\text { channel } 2 \text {, isoform } \\
\text { CRA_c OS=Homo } \\
\text { sapiens GN=VDAC2 PE=4 } \\
\text { SV=1 } \\
\text { (AOA024QZTO_HUMAN) }\end{array}$ & $\begin{array}{l}0.0007 \\
3\end{array}$ & Up \\
\hline $\begin{array}{l}\text { Acyl carrier protein, } \\
\text { mitochondrial OS=Homo } \\
\text { sapiens } G N=N D U F A B 1 \\
P E=1 \mathrm{SV}=3\end{array}$ & 0.0016 & Down & $\begin{array}{l}\text { Histone H2A.V } \\
\mathrm{OS}=\text { Homo sapiens } \\
\text { GN=H2AFV PE=1 SV=3 }\end{array}$ & $\begin{array}{l}0.0009 \\
9\end{array}$ & Up \\
\hline $\begin{array}{l}\text { Cluster of Epidermal } \\
\text { growth factor receptor } \\
\text { kinase substrate 8-like } \\
\text { protein } 1 \text { OS=Homo } \\
\text { sapiens GN=EPS8L1 PE=1 } \\
\text { SV=1 (B4DKV7_HUMAN) }\end{array}$ & 0.0017 & Down & $\begin{array}{l}\text { ATP synthase subunit } \\
\text { gamma } O S=H o m o \\
\text { sapiens } P E=2 S V=1\end{array}$ & $\begin{array}{l}0.0003 \\
4\end{array}$ & Up \\
\hline
\end{tabular}




\begin{tabular}{|c|c|c|c|c|c|}
\hline $\begin{array}{l}\text { Cluster of S100A10 } \\
\text { protein (Fragment) } \\
\text { OS=Homo sapiens } \\
\text { GN=S100A10 PE=2 SV=1 } \\
\text { (Q6FGE5_HUMAN) }\end{array}$ & 0.0023 & Down & $\begin{array}{l}\text { Cluster of } \\
\text { Methylcrotonoyl-CoA } \\
\text { carboxylase beta chain, } \\
\text { mitochondrial OS=Homo } \\
\text { sapiens GN=MCCC2 } \\
\text { PE=1 SV=1 } \\
\text { (MCCB_HUMAN) }\end{array}$ & $\begin{array}{l}0.0009 \\
6\end{array}$ & Up \\
\hline $\begin{array}{l}\text { Lactoylglutathione lyase } \\
\text { OS=Homo sapiens } \\
\text { GN=HEL-S-74 PE=2 SV=1 }\end{array}$ & 0.0022 & Down & $\begin{array}{l}\text { Cluster of } \\
\text { Transcriptional activator } \\
\text { protein Pur-alpha } \\
\text { OS=Homo sapiens } \\
\text { GN=PURA PE=1 SV=2 } \\
\text { (PURA_HUMAN) } \\
\end{array}$ & $\begin{array}{l}0.0009 \\
6\end{array}$ & Up \\
\hline $\begin{array}{l}\text { Cluster of Nuclear } \\
\text { autoantigenic sperm } \\
\text { protein OS=Homo } \\
\text { sapiens GN=NASP PE=1 } \\
\text { SV=2 (NASP_HUMAN) }\end{array}$ & 0.0021 & Down & $\begin{array}{l}\text { Dolichyl- } \\
\text { diphosphooligosacchari } \\
\text { de-protein } \\
\text { glycosyltransferase } \\
\text { subunit } 2 \text { OS=Homo } \\
\text { sapiens GN=RPN2 PE=1 } \\
\text { SV=3 }\end{array}$ & 0.001 & Up \\
\hline $\begin{array}{l}\text { Cluster of cDNA } \\
\text { FL76823, highly similar } \\
\text { to Homo sapiens splicing } \\
\text { factor, arginine/serine- } \\
\text { rich } 6 \text { (SFRS6), mRNA } \\
\text { OS=Homo sapiens PE=2 } \\
\text { SV=1 (A8K588_HUMAN) }\end{array}$ & 0.0023 & Down & $\begin{array}{l}\text { Isocitrate } \\
\text { dehydrogenase [NAD] } \\
\text { subunit, mitochondrial } \\
\text { OS=Homo sapiens } \\
\text { GN=IDH3B PE=1 SV=1 }\end{array}$ & $\begin{array}{l}0.0007 \\
5\end{array}$ & Up \\
\hline $\begin{array}{l}\text { Proteasome subunit beta } \\
\text { type } O S=\text { Homo sapiens } \\
P E=2 S V=1\end{array}$ & 0.002 & Down & $\begin{array}{l}\text { ATP-dependent 6- } \\
\text { phosphofructokinase, } \\
\text { liver type OS=Homo } \\
\text { sapiens GN=PFKL PE=1 } \\
\text { SV=6 }\end{array}$ & $\begin{array}{l}0.0004 \\
7\end{array}$ & Up \\
\hline $\begin{array}{l}\text { Spermine synthase } \\
\text { OS=Homo sapiens } \\
\text { GN=SMS PE=1 SV=2 }\end{array}$ & 0.0022 & Down & $\begin{array}{l}\text { cDNA FLJ52100 } \\
\text { OS=Homo sapiens } P E=2 \\
S V=1\end{array}$ & $\begin{array}{l}0.0003 \\
6\end{array}$ & Up \\
\hline $\begin{array}{l}\text { Succinate--CoA ligase } \\
\text { [GDP-forming] subunit } \\
\text { beta, mitochondrial } \\
\text { OS=Homo sapiens } \\
\text { GN=SUCLG2 PE=1 SV=2 }\end{array}$ & 0.0018 & Down & $\begin{array}{l}\text { SF3A2 protein } \\
\text { (Fragment) OS=Homo } \\
\text { sapiens } \mathrm{GN}=\mathrm{SF} 3 \mathrm{~A} 2 \mathrm{PE}=2 \\
\mathrm{SV}=1\end{array}$ & 0.0012 & Up \\
\hline $\begin{array}{l}\text { cDNA FL59206, highly } \\
\text { similar to Eukaryotic } \\
\text { translation initiation } \\
\text { factor 4B OS=Homo } \\
\text { sapiens PE=2 SV=1 }\end{array}$ & 0.0019 & Down & $\begin{array}{l}\text { Cytochrome } \mathrm{c} 1 \text {, heme } \\
\text { protein, mitochondrial } \\
\mathrm{OS}=\text { Homo sapiens } \\
\mathrm{GN}=\mathrm{CYC} 1 \mathrm{PE}=1 \mathrm{SV}=3\end{array}$ & 0.0013 & Up \\
\hline $\begin{array}{l}\text { Cluster of DDAH2 } \\
\text { OS=Homo sapiens } \\
\text { GN=HEL-S-277 PE=1 }\end{array}$ & 0.002 & Down & $\begin{array}{l}\text { Cluster of Epididymis } \\
\text { tissue sperm binding } \\
\text { protein Li 3a OS=Homo }\end{array}$ & 0.0015 & Up \\
\hline
\end{tabular}




\begin{tabular}{|c|c|c|c|c|c|}
\hline $\begin{array}{l}\text { SV=1 } \\
\text { (V9HW53_HUMAN) }\end{array}$ & & & $\begin{array}{l}\text { sapiens PE=1 SV=1 } \\
\text { (E9KL35_HUMAN) }\end{array}$ & & \\
\hline $\begin{array}{l}\text { Cluster of Inorganic } \\
\text { pyrophosphatase 2, } \\
\text { mitochondrial OS=Homo } \\
\text { sapiens GN=PPA2 PE=1 } \\
\text { SV=2 (IPYR2_HUMAN) }\end{array}$ & 0.0025 & Down & $\begin{array}{l}\text { Cluster of } \\
\text { Transmembrane } 9 \\
\text { superfamily member } \\
\text { OS=Homo sapiens } \\
\text { GN=SMBP PE=2 SV=1 } \\
\text { (Q96JZ5_HUMAN) }\end{array}$ & 0.0016 & Up \\
\hline $\begin{array}{l}\text { Cluster of Keratin, type I } \\
\text { cytoskeletal } 18 \\
\text { OS=Homo sapiens } \\
\text { GN=KRT18 PE=1 SV=2 } \\
\text { (K1C18_HUMAN) }\end{array}$ & 0.003 & Down & $\begin{array}{l}\text { RNA binding protein } \\
\text { (Autoantigenic, hnRNP- } \\
\text { associated with lethal } \\
\text { yellow) long isoform } \\
\text { variant (Fragment) } \\
\text { OS=Homo sapiens } \\
\text { GN=RALY PE=1 SV=1 }\end{array}$ & 0.0017 & Up \\
\hline $\begin{array}{l}\text { Cluster of Alpha-actinin- } \\
4 \text { OS=Homo sapiens } \\
\text { GN=ACTN4 PE=1 SV=2 } \\
\text { (ACTN4_HUMAN) }\end{array}$ & 0.0032 & Down & $\begin{array}{l}\text { Succinate--CoA ligase } \\
\text { [ADP/GDP-forming] } \\
\text { subunit alpha, } \\
\text { mitochondrial OS=Homo } \\
\text { sapiens } G N=S U C L G 1 \\
P E=1 S V=4\end{array}$ & 0.0017 & Up \\
\hline $\begin{array}{l}\text { Cluster of Peptidyl-prolyl } \\
\text { cis-trans isomerase A } \\
\text { OS=Homo sapiens } \\
\text { GN=PPIA PE=1 SV=2 } \\
\text { (PPIA_HUMAN) }\end{array}$ & 0.0029 & Down & $\begin{array}{l}\text { Cluster of Histone H2B } \\
\text { type } 1-\mathrm{J} O S=\text { Homo } \\
\text { sapiens GN=HIST1H2BJ } \\
\text { PE=1 SV=3 } \\
\text { (H2B1J_HUMAN) }\end{array}$ & 0.0021 & Up \\
\hline $\begin{array}{l}\text { 40S ribosomal protein } \\
\text { S12 OS=Homo sapiens } \\
\text { GN=RPS12 PE=1 SV=3 }\end{array}$ & 0.003 & Down & $\begin{array}{l}\text { Cluster of MHC class I } \\
\text { antigen (Fragment) } \\
\text { OS=Homo sapiens } \\
\text { GN=HLA-A PE=3 SV=1 } \\
\text { (E5BBI6_HUMAN) }\end{array}$ & 0.0022 & Up \\
\hline $\begin{array}{l}\text { Cluster of Eukaryotic } \\
\text { peptide chain release } \\
\text { factor GTP-binding } \\
\text { subunit ERF3A OS=Homo } \\
\text { sapiens GN=GSPT1 PE=1 } \\
\text { SV=1 (ERF3A_HUMAN) }\end{array}$ & 0.0027 & Down & $\begin{array}{l}\text { Cluster of Histone } 1, \\
\text { H1e OS=Homo sapiens } \\
\text { GN=HIST1H1E PE=2 } \\
\text { SV=1 (A3R0T8_HUMAN) }\end{array}$ & 0.002 & Up \\
\hline $\begin{array}{l}\text { Cluster of Dynein heavy } \\
\text { chain } 12 \text {, axonemal } \\
\text { OS=Homo sapiens } \\
\text { GN=DNAH12 PE=1 SV=2 } \\
\text { (E9PG32_HUMAN- } \\
\text { DECOY) }\end{array}$ & 0.0032 & Down & $\begin{array}{l}\text { RPL21 protein OS=Homo } \\
\text { sapiens } G N=R P L 21 \mathrm{PE}=2 \\
\mathrm{SV}=1\end{array}$ & 0.0022 & Up \\
\hline $\begin{array}{l}\text { Cluster of Putative } \\
\text { deoxyribonuclease } \\
\text { TATDN1 OS=Homo } \\
\text { sapiens GN=TATDN1 }\end{array}$ & 0.0032 & Down & $\begin{array}{l}\text { Cluster of Ras-related C3 } \\
\text { botulinum toxin } \\
\text { substrate } 1 \text { (Rho family, } \\
\text { small GTP binding } \\
\text { protein Rac1) OS=Homo }\end{array}$ & 0.0023 & Up \\
\hline
\end{tabular}




\begin{tabular}{|c|c|c|c|c|c|}
\hline $\begin{array}{l}\mathrm{PE}=1 \mathrm{SV}=2 \\
\text { (TATD1_HUMAN) }\end{array}$ & & & $\begin{array}{l}\text { sapiens GN=RAC1 PE=2 } \\
\text { SV=1 (A4D2P1_HUMAN) }\end{array}$ & & \\
\hline $\begin{array}{l}\text { Alcohol dehydrogenase } \\
{[\mathrm{NADP}(+)] \text { OS=Homo }} \\
\text { sapiens GN=AKR1A1 } \\
\mathrm{PE}=1 \mathrm{SV}=3\end{array}$ & 0.0032 & Down & $\begin{array}{l}\text { Cluster of TOB3 } \\
\text { OS=Homo sapiens PE=2 } \\
\text { SV=1 (Q96T67_HUMAN) }\end{array}$ & 0.0023 & Up \\
\hline $\begin{array}{l}\text { Cluster of } \\
\text { Triosephosphate } \\
\text { isomerase OS=Homo } \\
\text { sapiens GN=TPI1 PE=1 } \\
\text { SV=3 (TPIS_HUMAN) }\end{array}$ & 0.0035 & Down & $\begin{array}{l}\text { H/ACA } \\
\text { ribonucleoprotein } \\
\text { complex subunit } 3 \\
\text { OS=Homo sapiens } \\
\text { GN=NOP10 } P E=1 \mathrm{SV}=1\end{array}$ & 0.0019 & Up \\
\hline $\begin{array}{l}\text { Cluster of } 26 \mathrm{~S} \\
\text { proteasome non-ATPase } \\
\text { regulatory subunit } 4 \\
\text { OS=Homo sapiens } \\
\text { GN=PSMD4 PE=1 SV=1 } \\
\text { (PSMD4_HUMAN) }\end{array}$ & 0.0035 & Down & $\begin{array}{l}\text { Cluster of Mitochondrial } \\
\text { transcription factor } A \\
\text { OS=Homo sapiens PE=4 } \\
\text { SV=1 (E5KSX8_HUMAN) }\end{array}$ & 0.0018 & Up \\
\hline $\begin{array}{l}\text { Cluster of ADP- } \\
\text { ribosylation factor } 1 \\
\text { OS=Homo sapiens } \\
\text { GN=ARF1 PE=1 SV=2 } \\
\text { (ARF1_HUMAN) }\end{array}$ & 0.0036 & Down & $\begin{array}{l}\text { Cysteine-tRNA ligase, } \\
\text { cytoplasmic OS=Homo } \\
\text { sapiens GN=CARS PE=1 } \\
\text { SV=1 }\end{array}$ & 0.0021 & Up \\
\hline $\begin{array}{l}\text { cDNA FL54170, highly } \\
\text { similar to Cytosolic } \\
\text { nonspecific dipeptidase } \\
\text { OS=Homo sapiens PE=2 } \\
\text { SV=1 }\end{array}$ & 0.0037 & Down & $\begin{array}{l}\text { cDNA, FLJ96465, highly } \\
\text { similar to Homo sapiens } \\
\text { solute carrier family } 25 \\
\text { (mitochondrial } \\
\text { carrier; phosphate } \\
\text { carrier), member } 3 \\
\text { (SLC25A3), nuclear gene } \\
\text { encodingmitochondrial } \\
\text { protein, transcript } \\
\text { variant } 1 \mathrm{~b} . . . \mathrm{OS}=\text { Homo } \\
\text { sapiens } \mathrm{PE}=2 \mathrm{SV}=1\end{array}$ & 0.0025 & Up \\
\hline $\begin{array}{l}\text { Tumor protein D52 } \\
\text { OS=Homo sapiens } \\
\text { GN=TPD52 PE=1 SV=2 }\end{array}$ & 0.0037 & Down & $\begin{array}{l}\text { Cluster of Histone H2A } \\
\text { type } 3 \text { OS=Homo } \\
\text { sapiens GN=HIST3H2A } \\
\text { PE=1 SV=3 } \\
\text { (H2A3_HUMAN) }\end{array}$ & 0.0032 & Up \\
\hline $\begin{array}{l}\text { Serine-threonine kinase } \\
\text { receptor-associated } \\
\text { protein OS=Homo } \\
\text { sapiens GN=STRAP PE=1 } \\
\text { SV=1 }\end{array}$ & 0.0038 & Down & $\begin{array}{l}\text { Cluster of Histone H3.3 } \\
\text { OS=Homo sapiens } \\
\text { GN=H3F3A PE=1 SV=2 } \\
\text { (H33_HUMAN) }\end{array}$ & 0.0027 & Up \\
\hline $\begin{array}{l}\text { Cluster of T-complex } \\
\text { protein } 1 \text { subunit gamma } \\
\text { OS=Homo sapiens } P E=2 \\
\text { SV=1 (Q2TU64_HUMAN) }\end{array}$ & 0.0039 & Down & $\begin{array}{l}\text { Cluster of NPC-A-16 } \\
\text { OS=Homo sapiens } \\
\text { GN=RPL9 PE=2 SV=1 } \\
\text { (Q53Z07_HUMAN) }\end{array}$ & 0.003 & Up \\
\hline
\end{tabular}




\begin{tabular}{|c|c|c|c|c|c|}
\hline $\begin{array}{l}\text { Cluster of Nucleosome } \\
\text { assembly protein 1-like } \\
\text { 4, isoform CRA_b } \\
\text { OS=Homo sapiens } \\
\text { GN=NAP1L4 PE=3 SV=1 } \\
\text { (AOA024RCC9_HUMAN) }\end{array}$ & 0.0039 & Down & $\begin{array}{l}\text { Cluster of } \\
\text { Dehydrogenase/reducta } \\
\text { se SDR family member } \\
2 \text {, mitochondrial } \\
\text { OS=Homo sapiens } \\
\text { GN=DHRS2 PE=1 SV=4 } \\
\text { (DHRS2_HUMAN) }\end{array}$ & 0.0029 & Up \\
\hline $\begin{array}{l}\text { Eukaryotic translation } \\
\text { initiation factor } 3 \\
\text { subunit M OS=Homo } \\
\text { sapiens GN=EIF3M PE=1 } \\
\text { SV=1 }\end{array}$ & 0.0042 & Down & $\begin{array}{l}\text { Polyribonucleotide } \\
\text { nucleotidyltransferase } \\
1 \text {, mitochondrial } \\
\text { OS=Homo sapiens } \\
\text { GN=PNPT1 PE=1 SV=2 }\end{array}$ & 0.0028 & Up \\
\hline $\begin{array}{l}\text { Cluster of } 14-3-3 \text { protein } \\
\text { zeta/delta OS=Homo } \\
\text { sapiens GN=YWHAZ } \\
P E=1 \text { SV=1 } \\
\text { (1433Z_HUMAN) }\end{array}$ & 0.0043 & Down & $\begin{array}{l}\text { Cluster of Dolichyl- } \\
\text { diphosphooligosacchari } \\
\text { de-protein } \\
\text { glycosyltransferase } \\
\text { subunit } 1 \text { OS=Homo } \\
\text { sapiens GN=RPN1 PE=1 } \\
\text { SV=1 (RPN1_HUMAN) }\end{array}$ & 0.0028 & Up \\
\hline $\begin{array}{l}\text { Costars family protein } \\
\text { ABRACL OS=Homo } \\
\text { sapiens } G N=A B R A C L \\
P E=1 S V=1\end{array}$ & 0.0043 & Down & $\begin{array}{l}\text { Cluster of 60S ribosomal } \\
\text { protein L14 OS=Homo } \\
\text { sapiens GN=RPL14 PE=1 } \\
\text { SV=4 (RL14_HUMAN) }\end{array}$ & 0.0032 & Up \\
\hline $\begin{array}{l}\text { Cluster of cDNA, } \\
\text { FL95650, highly similar } \\
\text { to Homo sapiens } \\
\text { karyopherin (importin) } \\
\text { beta } 1 \text { (KPNB1), mRNA } \\
\text { OS=Homo sapiens PE=2 } \\
\text { SV=1 (B2RBR9_HUMAN) }\end{array}$ & 0.0045 & Down & $\begin{array}{l}\text { Cluster of } \\
\text { Phosphoenolpyruvate } \\
\text { carboxykinase [GTP], } \\
\text { mitochondrial OS=Homo } \\
\text { sapiens GN=PCK2 PE=1 } \\
\text { SV=3 (PCKGM_HUMAN) }\end{array}$ & 0.0028 & Up \\
\hline $\begin{array}{l}\text { Cluster of cDNA } \\
\text { FL53116, highly similar } \\
\text { to T-complex protein } 1 \\
\text { subunit epsilon } \\
\text { OS=Homo sapiens PE=2 } \\
\text { SV=1 (B4DZT5_HUMAN) }\end{array}$ & 0.0048 & Down & $\begin{array}{l}\text { Ubiquitin/ISG15- } \\
\text { conjugating enzyme E2 } \\
\text { L6 OS=Homo sapiens } \\
\text { GN=UBE2L6 PE=1 SV=4 }\end{array}$ & 0.0031 & Up \\
\hline $\begin{array}{l}\text { Solute carrier family } 2 \text {, } \\
\text { facilitated glucose } \\
\text { transporter member } 1 \\
\text { OS=Homo sapiens } \\
\text { GN=SLC2A1 PE=1 SV=2 }\end{array}$ & 0.0048 & Down & $\begin{array}{l}\text { cDNA FL75180, highly } \\
\text { similar to Homo sapiens } \\
\text { mitochondrial isoleucine } \\
\text { tRNA synthetase, mRNA } \\
\text { OS=Homo sapiens PE=2 } \\
\text { SV=1 }\end{array}$ & 0.0031 & Up \\
\hline $\begin{array}{l}\text { Myotrophin OS=Homo } \\
\text { sapiens } \mathrm{GN}=\mathrm{MTPN} P E=1 \\
\mathrm{SV}=2\end{array}$ & 0.0055 & Down & $\begin{array}{l}\text { AFG3-like protein } 2 \\
\text { OS=Homo sapiens } \\
\text { GN=AFG3L2 PE=1 SV=2 }\end{array}$ & 0.0026 & Up \\
\hline $\begin{array}{l}\text { Profilin-1 OS=Homo } \\
\text { sapiens GN=PFN1 PE=1 } \\
\mathrm{SV}=2\end{array}$ & 0.0059 & Down & $\begin{array}{l}\text { 39S ribosomal protein } \\
\text { L39, mitochondrial }\end{array}$ & 0.0034 & Up \\
\hline
\end{tabular}




\begin{tabular}{|c|c|c|c|c|c|}
\hline & & & $\begin{array}{l}\mathrm{OS}=\text { Homo sapiens } \\
\mathrm{GN}=\mathrm{MRPL} 39 \mathrm{PE}=1 \mathrm{SV}=3\end{array}$ & & \\
\hline $\begin{array}{l}\text { Testicular tissue protein } \\
\text { Li } 75 \text { OS=Homo sapiens } \\
\text { PE=2 SV=1 }\end{array}$ & 0.0059 & Down & $\begin{array}{l}\text { Cluster of Histone } \\
\text { deacetylase OS=Homo } \\
\text { sapiens GN=HDAC1 } \\
\text { PE=2 SV=1 } \\
\text { (Q6IT96_HUMAN) }\end{array}$ & 0.0035 & Up \\
\hline $\begin{array}{l}\text { Drebrin-like protein } \\
\text { OS=Homo sapiens } \\
G N=D B N L P E=1 S V=1\end{array}$ & 0.0059 & Down & $\begin{array}{l}\text { Cluster of G protein- } \\
\text { binding protein CRFG } \\
\text { variant (Fragment) } \\
\text { OS=Homo sapiens PE=2 } \\
\text { SV=1 (Q53GSO_HUMAN) }\end{array}$ & 0.0035 & Up \\
\hline $\begin{array}{l}\text { Cluster of HSPA1L } \\
\text { OS=Homo sapiens PE=3 } \\
\text { SV=1 } \\
\text { (A0A1U9X7X4_HUMAN) }\end{array}$ & 0.0063 & Down & $\begin{array}{l}\text { Leucine-rich repeat- } \\
\text { containing protein } 59 \\
\text { OS=Homo sapiens } \\
\text { GN=LRRC59 PE=1 SV=1 }\end{array}$ & 0.0037 & Up \\
\hline $\begin{array}{l}\text { Niban-like protein } 1 \\
\text { OS=Homo sapiens } \\
\text { GN=FAM129B PE=1 SV=3 }\end{array}$ & 0.0063 & Down & $\begin{array}{l}\text { Asparagine synthetase } \\
\text { [glutamine-hydrolyzing] } \\
\text { OS=Homo sapiens } \\
\text { GN=ASNS PE=1 SV=4 }\end{array}$ & 0.0044 & Up \\
\hline $\begin{array}{l}\text { Cluster of Nucleolar and } \\
\text { coiled-body } \\
\text { phosphoprotein } 1 \\
\text { OS=Homo sapiens } \\
\text { GN=NOLC1 PE=1 SV=2 } \\
\text { (NOLC1_HUMAN) }\end{array}$ & 0.0064 & Down & $\begin{array}{l}\text { Cluster of Calnexin } \\
\text { OS=Homo sapiens } \\
\text { GN=CANX PE=1 SV=2 } \\
\text { (CALX_HUMAN) }\end{array}$ & 0.0046 & Up \\
\hline $\begin{array}{l}\text { Serine/arginine } \\
\text { repetitive matrix } 1 \\
\text { isoform } 2 \text { (Fragment) } \\
\text { OS=Homo sapiens } \\
\text { GN=SRRM1 PE=2 SV=1 }\end{array}$ & 0.0064 & Down & $\begin{array}{l}\text { Septin-2 OS=Homo } \\
\text { sapiens GN=SEPT2 PE=1 } \\
\text { SV=1 }\end{array}$ & 0.0049 & Up \\
\hline $\begin{array}{l}\text { Malignant T-cell- } \\
\text { amplified sequence } 1 \\
\text { OS=Homo sapiens } \\
\text { GN=MCTS1 PE=1 SV=1 }\end{array}$ & 0.0062 & Down & $\begin{array}{l}\text { Lon protease homolog, } \\
\text { mitochondrial OS=Homo } \\
\text { sapiens } \mathrm{GN}=\mathrm{LONP1} \mathrm{PE}=2 \\
\mathrm{SV}=1\end{array}$ & 0.0053 & Up \\
\hline $\begin{array}{l}\text { Cluster of cDNA } \\
\text { FL32482 fis, clone } \\
\text { SKNMC2001324, highly } \\
\text { similar to Importin-4 } \\
\text { OS=Homo sapiens PE=2 } \\
\text { SV=1 (B3KPY9_HUMAN) }\end{array}$ & 0.0066 & Down & $\begin{array}{l}\text { Cluster of RPS4X protein } \\
\text { (Fragment) OS=Homo } \\
\text { sapiens GN=RPS4X PE=2 } \\
\text { SV=2 (Q96IR1_HUMAN) }\end{array}$ & 0.0054 & Up \\
\hline $\begin{array}{l}\text { Cluster of Hsp70-binding } \\
\text { protein } 1 \text { OS=Homo } \\
\text { sapiens GN=HSPBP1 } \\
\text { PE=1 SV=1 } \\
\text { (HPBP1_HUMAN) }\end{array}$ & 0.0065 & Down & $\begin{array}{l}\text { Cluster of cDNA } \\
\text { PSEC0016 fis, clone } \\
\text { NT2RM1001076, highly } \\
\text { similar to Procollagen- } \\
\text { lysine,2-oxoglutarate 5- } \\
\text { dioxygenase } 3 \text { (EC } \\
\text { 1.14.11.4) OS=Homo }\end{array}$ & 0.0055 & Up \\
\hline
\end{tabular}




\begin{tabular}{|c|c|c|c|c|c|}
\hline & & & $\begin{array}{l}\text { sapiens } \mathrm{PE}=2 \mathrm{SV}=1 \\
\text { (B3KQQ3_HUMAN) }\end{array}$ & & \\
\hline $\begin{array}{l}\text { Spectrin alpha chain, } \\
\text { non-erythrocytic } 1 \\
\text { OS=Homo sapiens } \\
\text { GN=SPTAN1 PE=1 SV=1 }\end{array}$ & 0.0069 & Down & $\begin{array}{l}\text { Topoisomerase (DNA) I } \\
\text { OS=Homo sapiens } \\
\text { GN=TOP1 PE=2 SV=1 }\end{array}$ & 0.0057 & Up \\
\hline $\begin{array}{l}\text { Cluster of Neuroblast } \\
\text { differentiation- } \\
\text { associated protein } \\
\text { AHNAK OS=Homo } \\
\text { sapiens GN=AHNAK PE=1 } \\
\text { SV=2 (AHNK_HUMAN) }\end{array}$ & 0.0075 & Down & $\begin{array}{l}\text { Ribosome-binding } \\
\text { protein } 1 \text { OS=Homo } \\
\text { sapiens GN=RRBP1 PE=1 } \\
\text { SV=1 }\end{array}$ & 0.0058 & Up \\
\hline $\begin{array}{l}\text { Nascent polypeptide- } \\
\text { associated complex } \\
\text { subunit alpha, muscle- } \\
\text { specific form OS=Homo } \\
\text { sapiens GN=NACA PE=1 } \\
\text { SV=1 }\end{array}$ & 0.0073 & Down & $\begin{array}{l}\text { Peptidyl-prolyl cis-trans } \\
\text { isomerase B OS=Homo } \\
\text { sapiens GN=PPIB PE=1 } \\
\text { SV=2 }\end{array}$ & 0.0064 & Up \\
\hline $\begin{array}{l}\text { Chromobox protein } \\
\text { homolog } 1 \text { OS=Homo } \\
\text { sapiens } \mathrm{GN}=\mathrm{CBX} 1 \mathrm{PE}=1 \\
\text { SV=1 }\end{array}$ & 0.0075 & Down & $\begin{array}{l}\text { Cluster of Small nuclear } \\
\text { ribonucleoprotein- } \\
\text { associated protein N } \\
\text { OS=Homo sapiens } \\
\text { GN=SNRPN PE=1 SV=1 } \\
\text { (RSMN_HUMAN) }\end{array}$ & 0.0064 & Up \\
\hline $\begin{array}{l}\text { Isocitrate } \\
\text { dehydrogenase [NADP] } \\
\text { OS=Homo sapiens PE=2 } \\
\text { SV=1 }\end{array}$ & 0.0078 & Down & $\begin{array}{l}\text { Cluster of } \\
\text { Chromodomain- } \\
\text { helicase-DNA-binding } \\
\text { protein } 4 \text { OS=Homo } \\
\text { sapiens GN=CHD4 PE=1 } \\
\text { SV=1 } \\
\text { (AOAOC4DGG9_HUMAN) }\end{array}$ & 0.0064 & Up \\
\hline $\begin{array}{l}\text { Cluster of Calmodulin-1 } \\
\text { OS=Homo sapiens } \\
\text { GN=CALM1 PE=1 SV=1 } \\
\text { (CALM1_HUMAN) }\end{array}$ & 0.0079 & Down & $\begin{array}{l}\text { 60S ribosomal protein } \\
\text { L35 OS=Homo sapiens } \\
\text { GN=RPL35 PE=1 SV=2 }\end{array}$ & 0.0066 & Up \\
\hline $\begin{array}{l}\text { Cluster of } \\
\text { Heterogeneous nuclear } \\
\text { ribonucleoprotein L } \\
\text { OS=Homo sapiens } \\
\text { GN=HNRNPL PE=1 SV=2 } \\
\text { (HNRPL_HUMAN) }\end{array}$ & 0.0084 & Down & $\begin{array}{l}\text { Trifunctional enzyme } \\
\text { subunit beta, } \\
\text { mitochondrial OS=Homo } \\
\text { sapiens GN=HADHB } \\
P E=1 \mathrm{SV}=3\end{array}$ & 0.0068 & Up \\
\hline $\begin{array}{l}\text { Endoribonuclease } \\
\text { LACTB2 OS=Homo } \\
\text { sapiens GN=LACTB2 } \\
\text { PE=1 SV=2 }\end{array}$ & 0.0089 & Down & $\begin{array}{l}\text { Cluster of cDNA } \\
\text { FL61290, highly similar } \\
\text { to Neutral alpha- } \\
\text { glucosidase AB } \\
\text { OS=Homo sapiens PE=2 } \\
\text { SV=1 (B4DJ30_HUMAN) }\end{array}$ & 0.0071 & Up \\
\hline
\end{tabular}




\begin{tabular}{|c|c|c|c|c|c|}
\hline $\begin{array}{l}\text { Cluster of Barrier to } \\
\text { autointegration factor } 1 \text {, } \\
\text { isoform CRA_a } \\
\text { OS=Homo sapiens } \\
\text { GN=BANF1 PE=4 SV=1 } \\
\text { (A0A024R5H0_HUMAN) }\end{array}$ & 0.0091 & Down & $\begin{array}{l}\text { Deoxynucleoside } \\
\text { triphosphate } \\
\text { triphosphohydrolase } \\
\text { SAMHD1 OS=Homo } \\
\text { sapiens GN=SAMHD1 } \\
\text { PE=1 SV=2 }\end{array}$ & 0.0074 & Up \\
\hline $\begin{array}{l}\text { Cluster of Epididymis } \\
\text { secretory protein Li } 85 \\
\text { OS=Homo sapiens } \\
\text { GN=PCBP1 PE=2 SV=1 } \\
\text { (Q53SS8_HUMAN) }\end{array}$ & 0.0092 & Down & $\begin{array}{l}\text { Testis derived transcript } \\
\text { ( } 3 \text { LIM domains) } \\
\text { OS=Homo sapiens } \\
\text { GN=TES PE=4 SV=1 }\end{array}$ & 0.0075 & Up \\
\hline $\begin{array}{l}\text { Cluster of Calponin } \\
\text { (Fragment) OS=Homo } \\
\text { sapiens PE=2 SV=1 } \\
\text { (Q53GK7_HUMAN) }\end{array}$ & 0.0094 & Down & $\begin{array}{l}\text { Cluster of ADP/ATP } \\
\text { translocase } 2 \text { OS=Homo } \\
\text { sapiens GN=SLC25A5 } \\
\text { PE=1 SV=7 } \\
\text { (ADT2_HUMAN) }\end{array}$ & 0.0079 & Up \\
\hline $\begin{array}{l}\text { BAG6 OS=Homo sapiens } \\
\text { GN=BAT3 PE=1 SV=1 }\end{array}$ & 0.01 & Down & $\begin{array}{l}\text { Cluster of GTP-binding } \\
\text { nuclear protein Ran } \\
\text { (Fragment) OS=Homo } \\
\text { sapiens GN=RAN PE=1 } \\
\text { SV=1 (J3KQE5_HUMAN) }\end{array}$ & 0.0082 & Up \\
\hline $\begin{array}{l}\text { Cluster of Keratin, type II } \\
\text { cytoskeletal } 8 \text { OS=Homo } \\
\text { sapiens GN=KRT8 PE=1 } \\
\text { SV=7 (K2C8_HUMAN) }\end{array}$ & 0.011 & Down & $\begin{array}{l}\text { Voltage-dependent } \\
\text { anion-selective channel } \\
\text { protein } 1 \mathrm{OS}=\text { Homo } \\
\text { sapiens } \mathrm{GN}=\mathrm{VDAC1} \mathrm{PE}=1 \\
\mathrm{SV}=2\end{array}$ & 0.0088 & Up \\
\hline $\begin{array}{l}\text { Fatty acid synthase } \\
\text { OS=Homo sapiens } \\
\text { GN=FASN PE=1 SV=3 }\end{array}$ & 0.011 & Down & $\begin{array}{l}\text { Adenylate kinase } 1 \\
\text { variant (Fragment) } \\
\mathrm{OS}=\text { Homo sapiens } \mathrm{PE}=2 \\
\mathrm{SV}=1\end{array}$ & 0.0088 & Up \\
\hline $\begin{array}{l}\text { Cluster of Cofilin-1 } \\
\text { OS=Homo sapiens } \\
\text { GN=CFL1 PE=1 SV=3 } \\
\text { (COF1_HUMAN) }\end{array}$ & 0.011 & Down & $\begin{array}{l}\text { Nodal modulator } 3 \\
\text { OS=Homo sapiens } \\
\text { GN=NOMO3 } P E=1 \text { SV=1 }\end{array}$ & 0.0089 & Up \\
\hline $\begin{array}{l}\text { Cluster of Transgelin-2 } \\
\text { OS=Homo sapiens } \\
\text { GN=TAGLN2 PE=1 SV=3 } \\
\text { (TAGL2_HUMAN) }\end{array}$ & 0.011 & Down & $\begin{array}{l}\text { 60S ribosomal protein } \\
\text { L11 OS=Homo sapiens } \\
\text { GN=RPL11 PE=1 SV=2 }\end{array}$ & 0.009 & Up \\
\hline $\begin{array}{l}\text { Splicing factor U2AF } 65 \\
\text { kDa subunit OS=Homo } \\
\text { sapiens GN=U2AF2 PE=1 } \\
\text { SV=4 }\end{array}$ & 0.011 & Down & $\begin{array}{l}\text { PGRMC1 protein } \\
\text { OS=Homo sapiens } \\
\text { GN=PGRMC1 } P E=2 \text { SV=1 }\end{array}$ & 0.0095 & Up \\
\hline $\begin{array}{l}\text { HDCMB21P OS=Homo } \\
\text { sapiens } P E=2 \text { SV=1 }\end{array}$ & 0.011 & Down & $\begin{array}{l}\text { Eukaryotic translation } \\
\text { initiation factor } 2 \text { beta } \\
\text { OS=Homo sapiens } \\
\text { GN=EIF2S2 PE=2 SV=1 }\end{array}$ & 0.0096 & Up \\
\hline $\begin{array}{l}\text { cDNA FL75699, highly } \\
\text { similar to Homo sapiens }\end{array}$ & 0.011 & Down & $\begin{array}{l}\text { Cluster of Basigin } \\
\text { OS=Homo sapiens }\end{array}$ & 0.0097 & Up \\
\hline
\end{tabular}




\begin{tabular}{|c|c|c|c|c|c|}
\hline $\begin{array}{l}\text { osteoclast stimulating } \\
\text { factor } 1 \text { (OSTF1), mRNA } \\
\text { OS=Homo sapiens PE=2 } \\
S V=1\end{array}$ & & & $\begin{array}{l}\mathrm{GN}=\mathrm{BSG} P \mathrm{PE}=1 \mathrm{SV}=2 \\
\text { (BASI_HUMAN) }\end{array}$ & & \\
\hline $\begin{array}{l}\text { Cluster of Annexin A1 } \\
\text { OS=Homo sapiens } \\
\text { GN=ANXA1 PE=1 SV=2 } \\
\text { (ANXA1_HUMAN) }\end{array}$ & 0.012 & Down & $\begin{array}{l}\text { 60S ribosomal protein } \\
\text { L24 OS=Homo sapiens } \\
\text { GN=RPL24 PE=1 SV=1 }\end{array}$ & 0.01 & Up \\
\hline $\begin{array}{l}\text { Cluster of BolA-like } \\
\text { protein } 2 \text { OS=Homo } \\
\text { sapiens GN=BOLA2 PE=1 } \\
\text { SV=2 } \\
\text { (A0A087WZT3_HUMAN) }\end{array}$ & 0.012 & Down & $\begin{array}{l}\text { Putative peripheral } \\
\text { benzodiazepine } \\
\text { receptor-related protein } \\
\text { (Fragment) OS=Homo } \\
\text { sapiens GN=TSPO PE=1 } \\
\text { SV=1 }\end{array}$ & 0.0099 & Up \\
\hline $\begin{array}{l}\text { Cluster of } \\
\text { Glyceraldehyde-3- } \\
\text { phosphate } \\
\text { dehydrogenase } \\
\text { OS=Homo sapiens } \\
\text { GN=GAPDH PE=1 SV=3 } \\
\text { (G3P_HUMAN) }\end{array}$ & 0.013 & Down & $\begin{array}{l}\text { NADPH--cytochrome } \\
\text { P450 reductase } \\
\text { OS=Homo sapiens } \\
\text { GN=POR PE=2 SV=1 }\end{array}$ & 0.01 & Up \\
\hline $\begin{array}{l}\text { Cluster of Protein } \\
\text { disulfide-isomerase } \\
\text { OS=Homo sapiens } \\
\text { GN=P4HB PE=2 SV=1 } \\
\text { (AOA024R8S5_HUMAN) }\end{array}$ & 0.013 & Down & $\begin{array}{l}\text { Cluster of Transketolase } \\
\text { variant (Fragment) } \\
\text { OS=Homo sapiens PE=2 } \\
\text { SV=1 } \\
\text { (Q53EM5_HUMAN) }\end{array}$ & 0.011 & Up \\
\hline $\begin{array}{l}\text { Cluster of Heat shock } \\
\text { 70kDa protein } 4 \text { isoform } \\
\text { a variant (Fragment) } \\
\text { OS=Homo sapiens PE=2 } \\
\text { SV=1 (Q59GF8_HUMAN) }\end{array}$ & 0.013 & Down & $\begin{array}{l}\text { Cluster of 2'-5'- } \\
\text { oligoadenylate } \\
\text { synthetase 3,100kDa, } \\
\text { isoform CRA_a } \\
\text { OS=Homo sapiens } \\
\text { GN=OAS3 PE=4 SV=1 } \\
\text { (A0A024RBQ5_HUMAN) }\end{array}$ & 0.011 & Up \\
\hline $\begin{array}{l}\text { Multifunctional } \\
\text { methyltransferase } \\
\text { subunit TRM112-like } \\
\text { protein OS=Homo } \\
\text { sapiens GN=TRMT112 } \\
\text { PE=1 SV=1 }\end{array}$ & 0.013 & Down & $\begin{array}{l}\text { Flavin reductase } \\
\text { (NADPH) OS=Homo } \\
\text { sapiens } \mathrm{GN}=\mathrm{BLVRB} P E=1 \\
\mathrm{SV}=3\end{array}$ & 0.011 & Up \\
\hline $\begin{array}{l}\text { Cluster of Tumor protein } \\
\text { D54 OS=Homo sapiens } \\
\text { GN=TPD52L2 PE=1 SV=1 } \\
\text { (A0A087WYR3_HUMAN) }\end{array}$ & 0.013 & Down & $\begin{array}{l}\text { Cluster of Glutamine- } \\
\text { fructose-phosphate } \\
\text { aminotransferase } \\
\text { [isomerizing] } 1 \\
\text { OS=Homo sapiens } \\
\text { GN=GFPT1 PE=1 SV=3 } \\
\text { (GFPT1_HUMAN) }\end{array}$ & 0.011 & Up \\
\hline
\end{tabular}




\begin{tabular}{|c|c|c|c|c|c|}
\hline $\begin{array}{l}\text { Testicular tissue protein } \\
\text { Li } 138 \text { OS=Homo sapiens } \\
\text { PE=2 SV=1 }\end{array}$ & 0.013 & Down & $\begin{array}{l}\text { cDNA, FLJ93510, highly } \\
\text { similar to Homo sapiens } \\
\text { JTV1 gene (JTV1), mRNA } \\
\text { OS=Homo sapiens PE=2 } \\
\text { SV=1 }\end{array}$ & 0.011 & Up \\
\hline $\begin{array}{l}\text { Synaptogyrin OS=Homo } \\
\text { sapiens } G N=S Y N G R 2 \\
P E=3 S V=1\end{array}$ & 0.014 & Down & $\begin{array}{l}\text { Lysine-tRNA ligase } \\
\text { OS=Homo sapiens } \\
\text { GN=KARS PE=1 SV=3 }\end{array}$ & 0.012 & Up \\
\hline $\begin{array}{l}\text { Cluster of ELAV-like } \\
\text { protein } 1 \mathrm{OS}=\text { Homo } \\
\text { sapiens GN=ELAVL1 PE=1 } \\
\text { SV=2 (ELAV1_HUMAN) }\end{array}$ & 0.015 & Down & $\begin{array}{l}\text { E3 ubiquitin-protein } \\
\text { ligase RNF213 OS=Homo } \\
\text { sapiens GN=RNF213 } \\
\text { PE=1 SV=1 }\end{array}$ & 0.012 & Up \\
\hline $\begin{array}{l}\text { Cluster of Cellular } \\
\text { retinoic acid-binding } \\
\text { protein } 2 \text { OS=Homo } \\
\text { sapiens GN=CRABP2 } \\
\text { PE=1 SV=2 } \\
\text { (RABP2_HUMAN) }\end{array}$ & 0.015 & Down & $\begin{array}{l}\text { Cluster of Cytochrome } \\
\text { b-c1 complex subunit 2, } \\
\text { mitochondrial OS=Homo } \\
\text { sapiens GN=UQCRC2 } \\
\text { PE=1 SV=3 } \\
\text { (QCR2_HUMAN) }\end{array}$ & 0.013 & Up \\
\hline $\begin{array}{l}\text { CDNA FL52068, highly } \\
\text { similar to Microtubule- } \\
\text { associated protein RP/EB } \\
\text { family member } 1 \\
\text { OS=Homo sapiens PE=2 } \\
\text { SV=1 }\end{array}$ & 0.015 & Down & $\begin{array}{l}\text { Cluster of cDNA } \\
\text { FL54020, highly similar } \\
\text { to Heterogeneous } \\
\text { nuclear } \\
\text { ribonucleoprotein U } \\
\text { OS=Homo sapiens PE=2 } \\
\text { SV=1 (B4DLR3_HUMAN) }\end{array}$ & 0.014 & Up \\
\hline $\begin{array}{l}\text { Cluster of SET OS=Homo } \\
\text { sapiens GN=SET PE=2 } \\
\text { SV=1 (Q5VXV3_HUMAN) }\end{array}$ & 0.015 & Down & $\begin{array}{l}\text { 40S ribosomal protein } \\
\text { S3a OS=Homo sapiens } \\
\text { GN=RPS3A PE=2 SV=1 }\end{array}$ & 0.014 & Up \\
\hline $\begin{array}{l}\text { Cluster of Nuclease } \\
\text { sensitive element } \\
\text { binding protein-1 } \\
\text { OS=Homo sapiens PE=2 } \\
\text { SV=1 (Q7KZ24_HUMAN) }\end{array}$ & 0.015 & Down & $\begin{array}{l}\text { Cluster of Core histone } \\
\text { macro-H2A.1 OS=Homo } \\
\text { sapiens GN=H2AFY PE=1 } \\
\text { SV=4 (H2AY_HUMAN) }\end{array}$ & 0.014 & Up \\
\hline $\begin{array}{l}\text { Cluster of La-related } \\
\text { protein } 1 \mathrm{OS}=\text { Homo } \\
\text { sapiens GN=LARP1 PE=1 } \\
\text { SV=2 (LARP1_HUMAN) }\end{array}$ & 0.015 & Down & $\begin{array}{l}\text { X-ray repair cross- } \\
\text { complementing protein } \\
6 \mathrm{OS}=\text { Homo sapiens } \\
\mathrm{GN}=\mathrm{XRCC6} \mathrm{PE}=1 \mathrm{SV}=1\end{array}$ & 0.015 & Up \\
\hline $\begin{array}{l}\text { Cluster of T-complex } \\
\text { protein } 1 \text { subunit beta } \\
\text { OS=Homo sapiens } \\
\text { GN=CCT2 PE=1 SV=4 } \\
\text { (TCPB_HUMAN) }\end{array}$ & 0.016 & Down & $\begin{array}{l}\text { Mitochondrial carrier } \\
\text { homolog } 2 \text { OS=Homo } \\
\text { sapiens } \mathrm{GN}=\mathrm{MTCH} 2 \\
\mathrm{PE}=1 \mathrm{SV}=1\end{array}$ & 0.015 & Up \\
\hline $\begin{array}{l}\text { Cluster of Histone- } \\
\text { binding protein RBBP7 } \\
\text { OS=Homo sapiens } \\
\text { GN=RBBP7 PE=1 SV=1 } \\
\text { (RBBP7_HUMAN) }\end{array}$ & 0.016 & Down & $\begin{array}{l}\text { Cluster of Isocitrate } \\
\text { dehydrogenase [NAD] } \\
\text { subunit, mitochondrial } \\
\text { OS=Homo sapiens } \\
\text { GN=IDH3G PE=1 SV=1 } \\
\text { (E7EQB8_HUMAN) }\end{array}$ & 0.015 & Up \\
\hline
\end{tabular}




\begin{tabular}{|c|c|c|c|c|c|}
\hline $\begin{array}{l}\text { Peptidyl-prolyl cis-trans } \\
\text { isomerase NIMA- } \\
\text { interacting } 1 \mathrm{OS}=\text { Homo } \\
\text { sapiens GN=PIN1 PE=1 } \\
\text { SV=1 }\end{array}$ & 0.016 & Down & $\begin{array}{l}\text { Prohibitin-2 OS=Homo } \\
\text { sapiens } \mathrm{GN}=\mathrm{PHB} 2 \mathrm{PE}=1 \\
\mathrm{SV}=1\end{array}$ & 0.016 & Up \\
\hline $\begin{array}{l}\text { Calreticulin variant } \\
\text { (Fragment) OS=Homo } \\
\text { sapiens } \mathrm{PE}=2 \mathrm{SV}=1\end{array}$ & 0.017 & Down & $\begin{array}{l}\text { Cluster of Interferon- } \\
\text { induced protein with } \\
\text { tetratricopeptide } \\
\text { repeats } 1 \text { OS=Homo } \\
\text { sapiens GN=IFIT1 PE=1 } \\
\text { SV=2 (IFIT1_HUMAN) }\end{array}$ & 0.016 & Up \\
\hline $\begin{array}{l}\text { Cluster of CSTB protein } \\
\text { OS=Homo sapiens } \\
\text { GN=CSTB PE=2 SV=1 } \\
\text { (Q76LA1_HUMAN) }\end{array}$ & 0.017 & Down & $\begin{array}{l}\text { Ubiquitin carboxyl- } \\
\text { terminal hydrolase } 14 \\
\text { OS=Homo sapiens } \\
\text { GN=USP14 PE=1 SV=2 }\end{array}$ & 0.016 & Up \\
\hline $\begin{array}{l}\text { Cluster of Far upstream } \\
\text { element-binding protein } \\
2 \text { OS=Homo sapiens } \\
\text { GN=KHSRP PE=1 SV=1 } \\
\text { (A0A087WTP3_HUMAN) }\end{array}$ & 0.017 & Down & $\begin{array}{l}\text { Cluster of Tubulin alpha } \\
\text { chain OS=Homo sapiens } \\
\text { GN=TUBA1C PE=1 SV=1 } \\
\text { (F5H5D3_HUMAN) }\end{array}$ & 0.017 & Up \\
\hline $\begin{array}{l}\text { Eukaryotic translation } \\
\text { initiation factor } 3 \\
\text { subunit } \mathrm{A} \text { OS=Homo } \\
\text { sapiens GN=EIF3A PE=1 } \\
\text { SV=1 }\end{array}$ & 0.017 & Down & $\begin{array}{l}\text { Cluster of Proteasome } \\
\text { subunit alpha type } \\
\text { OS=Homo sapiens } \\
\text { GN=PSMA6 PE=1 SV=1 } \\
\text { (G3V295_HUMAN) }\end{array}$ & 0.017 & Up \\
\hline $\begin{array}{l}\text { Proteasome subunit } \\
\text { alpha type } O S=H o m o \\
\text { sapiens } P E=2 S V=1\end{array}$ & 0.017 & Down & $\begin{array}{l}\text { DNA-dependent protein } \\
\text { kinase catalytic subunit } \\
\text { OS=Homo sapiens } \\
\text { GN=PRKDC } P E=1 \text { SV=3 }\end{array}$ & 0.017 & Up \\
\hline $\begin{array}{l}\text { EF-hand domain- } \\
\text { containing protein D2 } \\
\text { OS=Homo sapiens } \\
\text { GN=EFHD2 PE=1 SV=1 }\end{array}$ & 0.017 & Down & $\begin{array}{l}\text { Apoptosis-inducing } \\
\text { factor } 1 \text {, mitochondrial } \\
\text { OS=Homo sapiens } \\
\text { GN=AIFM1 PE=1 SV=1 }\end{array}$ & 0.018 & Up \\
\hline $\begin{array}{l}\text { cDNA FL56566, highly } \\
\text { similar to Small } \\
\text { glutamine-rich } \\
\text { tetratricopeptiderepeat- } \\
\text { containing protein A } \\
\text { OS=Homo sapiens PE=2 } \\
\text { SV=1 }\end{array}$ & 0.017 & Down & $\begin{array}{l}\text { Eukaryotic translation } \\
\text { initiation factor } 3 \\
\text { subunit D OS=Homo } \\
\text { sapiens GN=EIF3D PE=2 } \\
\text { SV=1 }\end{array}$ & 0.018 & Up \\
\hline $\begin{array}{l}\text { Cluster of Prosaposin } \\
\text { (Variant Gaucher disease } \\
\text { and variant } \\
\text { metachromatic } \\
\text { leukodystrophy), isoform } \\
\text { CRA_b OS=Homo sapiens } \\
\text { GN=PSAP PE=4 SV=1 } \\
\text { (AOA024QZQ2_HUMAN) }\end{array}$ & 0.017 & Down & $\begin{array}{l}\text { Bifunctional } \\
\text { glutamate/proline-tRNA } \\
\text { ligase OS=Homo sapiens } \\
\text { GN=EPRS PE=1 SV=5 }\end{array}$ & 0.019 & Up \\
\hline
\end{tabular}




\begin{tabular}{|c|c|c|c|c|c|}
\hline $\begin{array}{l}\text { Cluster of cDNA } \\
\text { FL55694, highly similar } \\
\text { to Dipeptidyl-peptidase } \\
1 \text { (EC 3.4.14.1) OS=Homo } \\
\text { sapiens PE=2 SV=1 } \\
\text { (B4DJQ8_HUMAN) }\end{array}$ & 0.018 & Down & $\begin{array}{l}\text { Histone } \mathrm{H} 1 \times \mathrm{OS}=\text { Homo } \\
\text { sapiens } \mathrm{GN}=\mathrm{H} 1 \mathrm{FX} P E=1 \\
\mathrm{SV}=1\end{array}$ & 0.019 & Up \\
\hline $\begin{array}{l}\text { Cluster of Myosin } \\
\text { regulatory light chain } \\
\text { 12A OS=Homo sapiens } \\
\text { GN=MYL12A PE=1 SV=2 } \\
\text { (ML12A_HUMAN) }\end{array}$ & 0.018 & Down & $\begin{array}{l}\text { Cluster of } 40 \text { S ribosomal } \\
\text { protein } \mathrm{S} 16 \text { OS=Homo } \\
\text { sapiens GN=RPS16 PE=1 } \\
\text { SV=2 (RS16_HUMAN) }\end{array}$ & 0.02 & Up \\
\hline $\begin{array}{l}\text { Drug-sensitive protein } 1 \\
\mathrm{OS}=\text { Homo sapiens } \\
\mathrm{GN}=\mathrm{YA} 61 \mathrm{PE}=2 \mathrm{SV}=1\end{array}$ & 0.018 & Down & $\begin{array}{l}\text { 60S ribosomal protein } \\
\text { L34 OS=Homo sapiens } \\
\text { GN=RPL34 PE=1 SV=3 }\end{array}$ & 0.02 & Up \\
\hline $\begin{array}{l}\text { Cluster of Myeloid- } \\
\text { derived growth factor } \\
\text { OS=Homo sapiens } \\
\text { GN=MYDGF PE=1 SV=1 } \\
\text { (MYDGF_HUMAN) }\end{array}$ & 0.018 & Down & $\begin{array}{l}\text { EPHX1 protein } \\
\text { (Fragment) OS=Homo } \\
\text { sapiens } \mathrm{GN}=\mathrm{EPHX1} \mathrm{PE}=2 \\
\mathrm{SV}=1\end{array}$ & 0.02 & Up \\
\hline $\begin{array}{l}\text { Cluster of Polypyrimidine } \\
\text { tract binding protein } 1 \text {, } \\
\text { isoform CRA_b } \\
\text { OS=Homo sapiens } \\
\text { GN=PTBP1 PE=1 SV=4 } \\
\text { (A6NLN1_HUMAN) }\end{array}$ & 0.019 & Down & $\begin{array}{l}\text { 60S ribosomal protein } \\
\text { L30 (Fragment) } \\
\text { OS=Homo sapiens } \\
\text { GN=RPL30 } \mathrm{PE}=1 \mathrm{SV}=1\end{array}$ & 0.021 & Up \\
\hline $\begin{array}{l}\text { Nuclear migration } \\
\text { protein nudC OS=Homo } \\
\text { sapiens GN=NUDC PE=1 } \\
\text { SV=1 }\end{array}$ & 0.019 & Down & $\begin{array}{l}\text { Flap endonuclease } 1 \\
\text { OS=Homo sapiens } \\
\text { GN=FEN1 PE=2 SV=1 }\end{array}$ & 0.021 & Up \\
\hline $\begin{array}{l}\text { Integrin alpha- } 6 \\
\text { OS=Homo sapiens } \\
\text { GN=ITGA6 PE=1 SV=5 }\end{array}$ & 0.019 & Down & $\begin{array}{l}\text { Cluster of } 40 \text { S ribosomal } \\
\text { protein } \mathrm{S} 19 \text { OS=Homo } \\
\text { sapiens GN=RPS19 PE=2 } \\
\text { SV=1 (B0ZBDO_HUMAN) }\end{array}$ & 0.024 & Up \\
\hline $\begin{array}{l}\text { Gamma- } \\
\text { glutamylcyclotransferase } \\
\text { OS=Homo sapiens } \\
\text { GN=GGCT PE=1 SV=1 }\end{array}$ & 0.019 & Down & $\begin{array}{l}\text { Cluster of 60S ribosomal } \\
\text { protein L17 OS=Homo } \\
\text { sapiens GN=RPL17 PE=1 } \\
\text { SV=3 (RL17_HUMAN) }\end{array}$ & 0.024 & Up \\
\hline $\begin{array}{l}\text { Annexin } \mathrm{A} 10 \mathrm{OS}=\text { Homo } \\
\text { sapiens } \mathrm{GN}=\mathrm{ANX} 10 \\
\mathrm{PE}=1 \mathrm{SV}=3\end{array}$ & 0.019 & Down & $\begin{array}{l}\text { Cluster of Serine } \\
\text { hydroxymethyltransfera } \\
\text { se, mitochondrial } \\
\text { (Fragment) OS=Homo } \\
\text { sapiens GN=SHMT2 } \\
\text { PE=1 SV=1 } \\
\text { (G3V4W5_HUMAN) }\end{array}$ & 0.025 & Up \\
\hline $\begin{array}{l}\text { Cluster of Elongation } \\
\text { factor 1-delta (Fragment) } \\
\text { OS=Homo sapiens }\end{array}$ & 0.02 & Down & $\begin{array}{l}\text { Regulator of } \\
\text { chromosome } \\
\text { condensation 2, isoform } \\
\text { CRA_a OS=Homo }\end{array}$ & 0.025 & Up \\
\hline
\end{tabular}




\begin{tabular}{|c|c|c|c|c|c|}
\hline $\begin{array}{l}\text { GN=EEF1D PE=1 SV=1 } \\
\text { (E9PIZ1_HUMAN) }\end{array}$ & & & $\begin{array}{l}\text { sapiens } \mathrm{GN}=\mathrm{RCC} 2 \mathrm{PE}=4 \\
\mathrm{SV}=1\end{array}$ & & \\
\hline $\begin{array}{l}\text { Cluster of Transcription } \\
\text { factor BTF3 OS=Homo } \\
\text { sapiens GN=BTF3 PE=1 } \\
\text { SV=1 (BTF3_HUMAN) }\end{array}$ & 0.02 & Down & $\begin{array}{l}\text { Cluster of Glutamate } \\
\text { dehydrogenase } \\
\text { OS=Homo sapiens PE=2 } \\
\text { SV=1 } \\
\text { (B4DMF5_HUMAN) }\end{array}$ & 0.025 & Up \\
\hline $\begin{array}{l}\text { Stathmin OS=Homo } \\
\text { sapiens } G N=S T M N 1 P E=2 \\
S V=1\end{array}$ & 0.02 & Down & $\begin{array}{l}\text { Histone } \mathrm{H} 1.0 \mathrm{OS}=\text { Homo } \\
\text { sapiens } \mathrm{GN}=\mathrm{H} 1 \mathrm{FO} \mathrm{PE}=1 \\
\mathrm{SV}=3\end{array}$ & 0.025 & Up \\
\hline $\begin{array}{l}\text { Cluster of } \\
\text { Translationally- } \\
\text { controlled tumor protein } \\
\text { (Fragment) OS=Homo } \\
\text { sapiens GN=TPT1 PE=1 } \\
\text { SV=1 (E9PJF7_HUMAN) }\end{array}$ & 0.02 & Down & $\begin{array}{l}\text { Phosphoserine } \\
\text { aminotransferase } \\
O S=\text { Homo sapiens } P E=2 \\
S V=1\end{array}$ & 0.025 & Up \\
\hline $\begin{array}{l}\text { Small nuclear } \\
\text { ribonucleoprotein } F \\
\text { OS=Homo sapiens } \\
\text { GN=SNRPF PE=1 SV=1 }\end{array}$ & 0.02 & Down & $\begin{array}{l}\text { Cluster of 60S ribosomal } \\
\text { protein } \mathrm{L} 13 \mathrm{OS}=\text { Homo } \\
\text { sapiens GN=RPL13 PE=1 } \\
\text { SV=1 (Q6NZ55_HUMAN) }\end{array}$ & 0.026 & Up \\
\hline $\begin{array}{l}\text { Cluster of Plectin } \\
\text { OS=Homo sapiens } \\
\text { GN=PLEC PE=1 SV=3 } \\
\text { (PLEC_HUMAN) }\end{array}$ & 0.021 & Down & $\begin{array}{l}\text { Cluster of Ribosomal } \\
\text { protein L19 OS=Homo } \\
\text { sapiens GN=RPL19 PE=1 } \\
\text { SV=1 (J3KTE4_HUMAN) }\end{array}$ & 0.027 & Up \\
\hline $\begin{array}{l}\text { Obg-like ATPase } 1 \\
\text { OS=Homo sapiens } \\
\text { GN=OLA1 PE=1 SV=2 }\end{array}$ & 0.021 & Down & $\begin{array}{l}\text { Cluster of Peroxisome } \\
\text { proliferator activated } \\
\text { receptor interacting } \\
\text { complex protein } \\
\text { OS=Homo sapiens } \\
\text { GN=PRIC295 PE=2 SV=1 } \\
\text { (E1NZA1_HUMAN) }\end{array}$ & 0.027 & Up \\
\hline $\begin{array}{l}\text { Cluster of Ran GTPase- } \\
\text { activating protein } 1 \\
\text { OS=Homo sapiens } \\
\text { GN=RANGAP1 PE=1 SV=1 } \\
\text { (RAGP1_HUMAN) }\end{array}$ & 0.021 & Down & $\begin{array}{l}\text { Cluster of cDNA } \\
\text { FL45395 fis, clone } \\
\text { BRHIP3027191, highly } \\
\text { similar to } 150 \mathrm{kDa} \\
\text { oxygen-regulated } \\
\text { protein (Orp150) } \\
\text { OS=Homo sapiens PE=2 } \\
\text { SV=1 (B3KXH0_HUMAN) }\end{array}$ & 0.028 & Up \\
\hline $\begin{array}{l}\text { Peflin OS=Homo sapiens } \\
\text { GN=PEF1 PE=1 SV=1 }\end{array}$ & 0.021 & Down & $\begin{array}{l}\text { Cluster of cDNA } \\
\text { FL76789, highly similar } \\
\text { to Homo sapiens } \\
\text { methionine-tRNA } \\
\text { synthetase (MARS), } \\
\text { mRNA OS=Homo } \\
\text { sapiens PE=2 SV=1 } \\
\text { (A8K492_HUMAN) }\end{array}$ & 0.028 & Up \\
\hline
\end{tabular}




\begin{tabular}{|c|c|c|c|c|c|}
\hline $\begin{array}{l}\text { cDNA FLJ7841, highly } \\
\text { similar to Homo sapiens } \\
\text { NADH dehydrogenase } \\
\text { (ubiquinone) } \\
\text { flavoprotein } 2,24 \mathrm{kDa} \\
\text { (NDUFV2), mRNA } \\
\text { OS=Homo sapiens PE=2 } \\
\text { SV=1 }\end{array}$ & 0.022 & Down & $\begin{array}{l}\text { 2'-5'-oligoadenylate } \\
\text { synthase } 2 \text { OS=Homo } \\
\text { sapiens GN=OAS2 PE=1 } \\
\text { SV=1 }\end{array}$ & 0.028 & Up \\
\hline $\begin{array}{l}\text { SH3 domain binding } \\
\text { glutamic acid-rich } \\
\text { protein like 3, isoform } \\
\text { CRA_a (Fragment) } \\
\text { OS=Homo sapiens } \\
\text { GN=SH3BGRL3 PE=4 } \\
\text { SV=1 }\end{array}$ & 0.023 & Down & $\begin{array}{l}\text { Emerin } \mathrm{OS}=\text { Homo } \\
\text { sapiens } \mathrm{GN}=\mathrm{EMD} P E=1 \\
\mathrm{SV}=1\end{array}$ & 0.028 & Up \\
\hline $\begin{array}{l}\text { Cluster of Ran-binding } \\
\text { protein } 6 \text { OS=Homo } \\
\text { sapiens GN=RANBP6 } \\
\text { PE=1 SV=2 } \\
\text { (RNBP6_HUMAN) }\end{array}$ & 0.023 & Down & $\begin{array}{l}\text { Cluster of 60S ribosomal } \\
\text { protein } L 7 \text { a OS=Homo } \\
\text { sapiens GN=RPL7A PE=1 } \\
\text { SV=2 (RL7A_HUMAN) }\end{array}$ & 0.029 & Up \\
\hline $\begin{array}{l}\text { Cluster of UMP-CMP } \\
\text { kinase OS=Homo sapiens } \\
\text { GN=CMPK1 PE=1 SV=3 } \\
\text { (KCY_HUMAN) }\end{array}$ & 0.024 & Down & $\begin{array}{l}\text { Cluster of CTNND1 } \\
\text { protein (Fragment) } \\
\text { OS=Homo sapiens } \\
\text { GN=CTNND1 PE=2 SV=2 } \\
\text { (Q96FS1_HUMAN) }\end{array}$ & 0.029 & Up \\
\hline $\begin{array}{l}\text { Sorting nexin } 1 \text { isoform a } \\
\text { variant (Fragment) } \\
O S=H \text { Homo sapiens } P E=2 \\
S V=1\end{array}$ & 0.024 & Down & $\begin{array}{l}\text { 39S ribosomal protein } \\
\text { L4, mitochondrial } \\
\text { OS=Homo sapiens } \\
\text { GN=MRPL4 PE=1 SV=1 }\end{array}$ & 0.029 & Up \\
\hline $\begin{array}{l}\text { Cytochrome c oxidase } \\
\text { subunit } 5 \mathrm{~A} \text {, } \\
\text { mitochondrial } O S=\text { Homo } \\
\text { sapiens } \mathrm{GN}=\mathrm{COX} 5 \mathrm{~A} P E=1 \\
\text { SV=2 }\end{array}$ & 0.025 & Down & $\begin{array}{l}\text { Cluster of Ribosomal } \\
\text { protein L23, isoform } \\
\text { CRA_b OS=Homo } \\
\text { sapiens GN=RPL23 PE=3 } \\
\text { SV=1 } \\
\text { (AOA024R1Q8_HUMAN) }\end{array}$ & 0.03 & Up \\
\hline $\begin{array}{l}\text { Leucine-rich repeat- } \\
\text { containing protein } 47 \\
\text { OS=Homo sapiens } \\
\text { GN=LRRC47 PE=1 SV=1 }\end{array}$ & 0.025 & Down & $\begin{array}{l}\text { Leucine-rich PPR-motif } \\
\text { containing OS=Homo } \\
\text { sapiens GN=LRPPRC } \\
P E=4 \mathrm{SV}=1\end{array}$ & 0.03 & Up \\
\hline $\begin{array}{l}\text { Acyl-CoA-binding protein } \\
\text { OS=Homo sapiens } \\
\text { GN=DBI PE=1 SV=2 }\end{array}$ & 0.025 & Down & $\begin{array}{l}\text { ATP synthase subunit g, } \\
\text { mitochondrial OS=Homo } \\
\text { sapiens GN=ATP5L PE=1 } \\
\text { SV=3 }\end{array}$ & 0.03 & Up \\
\hline $\begin{array}{l}\text { Splicing factor 3B } \\
\text { subunit } 5 \mathrm{OS}=\text { Homo } \\
\text { sapiens } \mathrm{GN}=\mathrm{SF} 3 \mathrm{~B} 5 \mathrm{PE}=1 \\
\mathrm{SV}=1\end{array}$ & 0.025 & Down & $\begin{array}{l}\text { Cluster of Signal } \\
\text { recognition particle } \\
\text { subunit SRP72 } \\
\text { OS=Homo sapiens }\end{array}$ & 0.03 & Up \\
\hline
\end{tabular}




\begin{tabular}{|c|c|c|c|c|c|}
\hline & & & $\begin{array}{l}\text { GN=HEL103 PE=2 SV=1 } \\
\text { (V9HWK0_HUMAN) }\end{array}$ & & \\
\hline $\begin{array}{l}\text { Ribosomal protein, large, } \\
\text { P1, isoform CRA_a } \\
\text { OS=Homo sapiens } \\
\text { GN=RPLP1 PE=3 SV=1 }\end{array}$ & 0.026 & Down & $\begin{array}{l}\text { Protein NipSnap } \\
\text { homolog } 2 \text { OS=Homo } \\
\text { sapiens GN=NIPSNAP2 } \\
\text { PE=1 SV=1 }\end{array}$ & 0.03 & Up \\
\hline $\begin{array}{l}\text { HCG26477 OS=Homo } \\
\text { sapiens GN=RPS28 PE=2 } \\
\text { SV=1 }\end{array}$ & 0.026 & Down & $\begin{array}{l}\text { 40S ribosomal protein } \\
\text { S11 OS=Homo sapiens } \\
\text { GN=RPS11 PE=1 SV=3 }\end{array}$ & 0.031 & Up \\
\hline $\begin{array}{l}\text { Asparagine-tRNA ligase, } \\
\text { cytoplasmic OS=Homo } \\
\text { sapiens GN=NARS PE=1 } \\
\text { SV=1 }\end{array}$ & 0.026 & Down & $\begin{array}{l}\text { Cluster of Protein } \\
\text { NipSnap homolog } 1 \\
\text { OS=Homo sapiens } \\
\text { GN=NIPSNAP1 PE=1 } \\
\text { SV=1 (NIPS1_HUMAN) }\end{array}$ & 0.031 & Up \\
\hline $\begin{array}{l}\text { Cluster of Epiplakin } \\
\text { OS=Homo sapiens } \\
\text { GN=EPPK1 PE=1 SV=1 } \\
\text { (A0A087X1U6_HUMAN) }\end{array}$ & 0.027 & Down & $\begin{array}{l}\text { Cluster of Peptidyl-prolyl } \\
\text { cis-trans isomerase } \\
\text { OS=Homo sapiens PE=2 } \\
\text { SV=1 (B2R6X6_HUMAN) }\end{array}$ & 0.031 & Up \\
\hline $\begin{array}{l}\text { Alanine-tRNA ligase, } \\
\text { cytoplasmic OS=Homo } \\
\text { sapiens GN=AARS PE=1 } \\
\text { SV=2 }\end{array}$ & 0.027 & Down & $\begin{array}{l}\text { Cytochrome c oxidase } \\
\text { subunit } 6 C \text { OS=Homo } \\
\text { sapiens } G N=C O X 6 C P E=1 \\
S V=2\end{array}$ & 0.031 & Up \\
\hline $\begin{array}{l}\text { Cluster of cDNA } \\
\text { FL60127, highly similar } \\
\text { to Ubiquilin-2 OS=Homo } \\
\text { sapiens PE=2 SV=1 } \\
\text { (B4DM19_HUMAN) }\end{array}$ & 0.029 & Down & $\begin{array}{l}\text { Cluster of Citrate } \\
\text { synthase OS=Homo } \\
\text { sapiens GN=CS PE=1 } \\
\text { SV=1 (B4DJV2_HUMAN) }\end{array}$ & 0.032 & Up \\
\hline $\begin{array}{l}\text { SARS protein OS=Homo } \\
\text { sapiens } G N=S A R S P E=2 \\
S V=1\end{array}$ & 0.029 & Down & $\begin{array}{l}\text { Heterochromatin } \\
\text { protein 1-binding } \\
\text { protein } 3 \text { OS=Homo } \\
\text { sapiens GN=HP1BP3 } \\
\text { PE=1 SV=1 }\end{array}$ & 0.032 & Up \\
\hline $\begin{array}{l}\text { Cluster of Eukaryotic } \\
\text { translation initiation } \\
\text { factor } 4 \mathrm{H} \text { OS=Homo } \\
\text { sapiens GN=EIF4H PE=1 } \\
\text { SV=5 (IF4H_HUMAN) }\end{array}$ & 0.03 & Down & $\begin{array}{l}\text { Cluster of RPL26 protein } \\
\text { OS=Homo sapiens } \\
\text { GN=RPL26 PE=2 SV=1 } \\
\text { (Q6IBH6_HUMAN) }\end{array}$ & 0.033 & Up \\
\hline $\begin{array}{l}\text { cDNA FLJ6387, highly } \\
\text { similar to Homo sapiens } \\
\text { splicing factor, } \\
\text { arginine/serine-rich } 9 \\
\text { (SFRS9), mRNA } \\
\text { OS=Homo sapiens PE=2 } \\
\text { SV=1 }\end{array}$ & 0.031 & Down & $\begin{array}{l}\text { Regulation of nuclear } \\
\text { pre-mRNA domain- } \\
\text { containing protein 1B } \\
\text { OS=Homo sapiens } \\
\text { GN=RPRD1B PE=1 SV=1 }\end{array}$ & 0.033 & Up \\
\hline $\begin{array}{l}182 \text { kDa tankyrase-1- } \\
\text { binding protein } \\
\text { OS=Homo sapiens }\end{array}$ & 0.031 & Down & $\begin{array}{l}\text { Mago nashi protein } \\
\text { OS=Homo sapiens } \\
\text { GN=FLJ10292 PE=2 SV=1 }\end{array}$ & 0.033 & Up \\
\hline
\end{tabular}




\begin{tabular}{|c|c|c|c|c|c|}
\hline $\begin{array}{l}\mathrm{GN}=\mathrm{TNKS1BP1} \mathrm{PE}=1 \\
\mathrm{SV}=4\end{array}$ & & & & & \\
\hline $\begin{array}{l}\text { Splicing factor } \\
\text { arginine/serine-rich } 3 \\
\text { OS=Homo sapiens } \\
\text { GN=SFRS3 PE=2 SV=1 }\end{array}$ & 0.032 & Down & $\begin{array}{l}\text { Tryptophan--tRNA } \\
\text { ligase, cytoplasmic } \\
\text { OS=Homo sapiens } \\
\text { GN=WARS PE=1 SV=2 }\end{array}$ & 0.034 & Up \\
\hline $\begin{array}{l}\text { Cluster of Epididymis } \\
\text { secretory protein Li } 102 \\
\text { OS=Homo sapiens } \\
\text { GN=HEL-S-102 PE=2 } \\
\text { SV=1 } \\
\text { (V9HW43_HUMAN) }\end{array}$ & 0.032 & Down & $\begin{array}{l}\text { Calcium load-activated } \\
\text { calcium channel } \\
\text { OS=Homo sapiens } \\
\mathrm{GN}=\mathrm{TMCO} 1 \mathrm{PE}=2 \mathrm{SV}=1\end{array}$ & 0.034 & Up \\
\hline $\begin{array}{l}\text { Methionine } \\
\text { aminopeptidase } 2 \\
\text { OS=Homo sapiens } \\
\text { GN=METAP2 PE=1 SV=1 }\end{array}$ & 0.032 & Down & $\begin{array}{l}\text { Cluster of Protein PML } \\
\text { OS=Homo sapiens } \\
\text { GN=PML PE=1 SV=1 } \\
\text { (H3BT57_HUMAN) }\end{array}$ & 0.035 & Up \\
\hline $\begin{array}{l}\text { Cluster of Epithelial- } \\
\text { splicing regulatory } \\
\text { protein } 1 \text { (Fragment) } \\
\text { OS=Homo sapiens } \\
\text { GN=ESRP1 PE=1 SV=1 } \\
\text { (HOYBR2_HUMAN) }\end{array}$ & 0.032 & Down & $\begin{array}{l}\text { Cluster of } 40 \text { S ribosomal } \\
\text { protein } \mathrm{S} 2 \text { (Fragment) } \\
\text { OS=Homo sapiens } \\
\text { GN=RPS2 PE=1 SV=1 } \\
\text { (E9PMM9_HUMAN) }\end{array}$ & 0.036 & Up \\
\hline $\begin{array}{l}\mathrm{Na}(+) / \mathrm{H}(+) \text { exchange } \\
\text { regulatory cofactor } \\
\text { NHERF1 OS=Homo } \\
\text { sapiens GN=SLC9A3R1 } \\
\mathrm{PE}=1 \mathrm{SV}=4\end{array}$ & 0.033 & Down & $\begin{array}{l}\text { Cluster of DNA helicase } \\
\text { (Fragment) OS=Homo } \\
\text { sapiens PE=2 SV=1 } \\
\text { (Q53FG5_HUMAN) }\end{array}$ & 0.036 & Up \\
\hline $\begin{array}{l}\text { Cluster of Calpain small } \\
\text { subunit } 1 \text { OS=Homo } \\
\text { sapiens GN=CAPNS1 } \\
P E=1 \text { SV=1 } \\
\text { (A0A0C4DGQ5_HUMAN) }\end{array}$ & 0.034 & Down & $\begin{array}{l}\text { cDNA FL54723, highly } \\
\text { similar to Poly (ADP- } \\
\text { ribose) polymerase } 9 \\
\text { (EC } 2.4 .2 .30 \text { ) OS=Homo } \\
\text { sapiens } \mathrm{PE}=2 \mathrm{SV}=1\end{array}$ & 0.036 & Up \\
\hline $\begin{array}{l}\text { Ran-specific GTPase } \\
\text { activating protein } \\
\text { OS=Homo sapiens } \\
\text { GN=RANBP1 } \mathrm{PE}=1 \mathrm{SV}=1\end{array}$ & 0.035 & Down & $\begin{array}{l}\text { Cluster of 2- } \\
\text { oxoglutarate } \\
\text { dehydrogenase, } \\
\text { mitochondrial OS=Homo } \\
\text { sapiens GN=OGDH PE=1 } \\
\text { SV=1 } \\
\text { (AOAOD9SFS3_HUMAN) }\end{array}$ & 0.037 & Up \\
\hline $\begin{array}{l}\text { Cluster of DNA damage } \\
\text { Einding protein } 1 \\
\text { OS=Homo sapiens } \\
\text { GN=DDB1 PE=1 SV=1 } \\
\text { (DDB1_HUMAN) }\end{array}$ & 0.035 & Down & $\begin{array}{l}\text { Delta-1-pyrroline 5- } \\
\text { carboxylate synthase } \\
\text { OS=Homo sapiens } \\
\text { GN=ALDH18A1 PE=1 } \\
\text { SV=2 }\end{array}$ & 0.038 & Up \\
\hline $\begin{array}{l}\text { Cluster of Pyruvate } \\
\text { kinase OS=Homo sapiens } \\
\text { GN=PKM PE=1 SV=1 } \\
\text { (B4DNK4_HUMAN) }\end{array}$ & 0.036 & Down & $\begin{array}{l}\text { cDNA FL76962, highly } \\
\text { similar to Homo sapiens } \\
\text { nucleolar protein 5A } \\
\text { (56kDa with KKE/D }\end{array}$ & 0.038 & Up \\
\hline
\end{tabular}




\begin{tabular}{|c|c|c|c|c|c|}
\hline & & & $\begin{array}{l}\text { repeat) (NOL5A), mRNA } \\
O S=\text { Homo sapiens } P E=2 \\
S V=1\end{array}$ & & \\
\hline $\begin{array}{l}\text { Adenylosuccinate } \\
\text { synthetase isozyme } 2 \\
\text { OS=Homo sapiens } \\
\text { GN=ADSS PE=1 SV=3 }\end{array}$ & 0.036 & Down & $\begin{array}{l}\text { cDNA FL13654 fis, clone } \\
\text { PLACE1011477, highly } \\
\text { similar to Sorting nexin- } \\
2 \text { OS=Homo sapiens } \\
\text { PE=2 SV=1 }\end{array}$ & 0.039 & Up \\
\hline $\begin{array}{l}\text { Cluster of T-complex } \\
\text { protein } 1 \text { subunit delta } \\
\text { OS=Homo sapiens PE=2 } \\
\text { SV=1 (A8K3C3_HUMAN) }\end{array}$ & 0.037 & Down & $\begin{array}{l}\text { 40S ribosomal protein } \\
\text { S8 OS=Homo sapiens } \\
\text { GN=RPS8 PE=2 SV=1 }\end{array}$ & 0.04 & Up \\
\hline $\begin{array}{l}\text { Cluster of } \\
\text { Serine/threonine protein } \\
\text { phosphatase } 2 \mathrm{~A} 65 \mathrm{kDa} \\
\text { regulatory subunit A } \\
\text { alpha isoform OS=Homo } \\
\text { sapiens GN=PPP2R1A } \\
\mathrm{PE}=1 \mathrm{SV}=4 \\
(2 \mathrm{AAA} \text { HUMAN) }\end{array}$ & 0.037 & Down & $\begin{array}{l}\text { Splicing factor } 3 B \\
\text { subunit } 1 \mathrm{OS}=\text { Homo } \\
\text { sapiens GN=SF3B1 PE=1 } \\
\text { SV=3 }\end{array}$ & 0.04 & Up \\
\hline $\begin{array}{l}\text { Protein kinase } \mathrm{C} \\
\text { substrate } 80 \mathrm{~K}-\mathrm{H} \text {, isoform } \\
\text { CRA_a OS=Homo sapiens } \\
\text { GN=PRKCSH PE=4 SV=1 }\end{array}$ & 0.037 & Down & $\begin{array}{l}\text { 40S ribosomal protein } \\
\text { S24 OS=Homo sapiens } \\
\text { GN=RPS24 PE=1 SV=1 }\end{array}$ & 0.041 & Up \\
\hline $\begin{array}{l}\text { Cluster of } \\
\text { Methylenetetrahydrofol } \\
\text { ate dehydrogenase } \\
\text { (NADP+ dependent) 1, } \\
\text { methenyltetrahydrofolat } \\
\text { e cyclohydrolase, } \\
\text { formyltetrahydrofolate } \\
\text { synthetase, isoform } \\
\text { CRA_a OS=Homo sapiens } \\
\text { GN=MTHFD1 PE=3 SV=1 } \\
\text { (AOA024R652_HUMAN) }\end{array}$ & 0.038 & Down & $\begin{array}{l}\text { Cluster of Protein } \\
\text { FAM162A OS=Homo } \\
\text { sapiens GN=FAM162A } \\
\text { PE=1 SV=2 } \\
\text { (F162A_HUMAN) }\end{array}$ & 0.042 & Up \\
\hline $\begin{array}{l}\text { RcNSEP1 (Fragment) } \\
\text { OS=Homo sapiens } \mathrm{PE}=4 \\
\mathrm{SV}=1\end{array}$ & 0.038 & Down & $\begin{array}{l}\text { Procollagen } \\
\text { galactosyltransferase } 1 \\
\mathrm{OS}=\text { Homo sapiens } \\
\mathrm{GN}=\text { COLGALT1 } \mathrm{PE}=1 \\
\mathrm{SV}=1\end{array}$ & 0.042 & Up \\
\hline $\begin{array}{l}\text { Cluster of S-methyl-5'- } \\
\text { thioadenosine } \\
\text { phosphorylase OS=Homo } \\
\text { sapiens GN=MTAP PE=2 } \\
\text { SV=1 (Q6FHT1_HUMAN) }\end{array}$ & 0.038 & Down & $\begin{array}{l}\text { Catenin beta-1 } \\
\text { OS=Homo sapiens } \\
\text { GN=CTNNB1 PE=1 SV=1 }\end{array}$ & 0.043 & Up \\
\hline $\begin{array}{l}\text { Cluster of } \\
\text { Dimethylarginine } \\
\text { dimethylaminohydrolase }\end{array}$ & 0.038 & Down & $\begin{array}{l}\text { Cluster of elF2AK2 } \\
\text { protein OS=Homo } \\
\text { sapiens GN=EIF2AK2 }\end{array}$ & 0.045 & Up \\
\hline
\end{tabular}




\begin{tabular}{|c|c|c|c|c|c|}
\hline $\begin{array}{l}\text { 1, isoform CRA_b } \\
\text { OS=Homo sapiens } \\
\text { GN=DDAH1 PE=2 SV=1 } \\
\text { (B1AKK2_HUMAN) }\end{array}$ & & & $\begin{array}{l}\mathrm{PE}=2 \mathrm{SV}=1 \\
\text { (B7ZKK7_HUMAN) }\end{array}$ & & \\
\hline $\begin{array}{l}\text { Cluster of Adenylyl } \\
\text { cyclase associated } \\
\text { protein OS=Homo } \\
\text { sapiens PE=2 SV=1 } \\
\text { (B2RDY9_HUMAN) }\end{array}$ & 0.039 & Down & $\begin{array}{l}\text { Cluster of Cathepsin D } \\
\text { OS=Homo sapiens } \\
\text { GN=CTSD PE=1 SV=1 } \\
\text { (AOA1B0GVD5_HUMAN) }\end{array}$ & 0.046 & Up \\
\hline $\begin{array}{l}\text { Prefoldin subunit } 2 \\
\text { OS=Homo sapiens } \\
\text { GN=PFDN2 PE=1 SV=1 }\end{array}$ & 0.039 & Down & $\begin{array}{l}\text { tRNA-splicing ligase RtcB } \\
\text { homolog OS=Homo } \\
\text { sapiens GN=RTCB PE=1 } \\
\text { SV=1 }\end{array}$ & 0.046 & Up \\
\hline $\begin{array}{l}\text { Testicular tissue protein } \\
\text { Li } 198 \text { OS=Homo sapiens } \\
\text { PE=2 SV=1 }\end{array}$ & 0.04 & Down & $\begin{array}{l}\text { MRE11 meiotic } \\
\text { recombination } 11 \\
\text { homolog A ( } \mathrm{S} \text {. } \\
\text { cerevisiae), isoform } \\
\text { CRA_a OS=Homo } \\
\text { sapiens GN=MRE11A } \\
\text { PE=3 SV=1 }\end{array}$ & 0.046 & Up \\
\hline $\begin{array}{l}\text { Thioredoxin domain- } \\
\text { containing protein } 17 \\
\text { OS=Homo sapiens } \\
\text { GN=TXNDC17 PE=1 SV=1 }\end{array}$ & 0.04 & Down & $\begin{array}{l}\text { Tripeptidyl peptidase I, } \\
\text { isoform CRA_a } \\
\text { OS=Homo sapiens } \\
\text { GN=TPP1 PE=4 SV=1 }\end{array}$ & 0.047 & Up \\
\hline $\begin{array}{l}\text { Ubiquitin-conjugating } \\
\text { enzyme E2 variant } 2 \\
\text { (Fragment) OS=Homo } \\
\text { sapiens } \mathrm{GN}=\mathrm{UBE} 2 \mathrm{~V} 2 \\
\mathrm{PE}=1 \mathrm{SV}=1\end{array}$ & 0.04 & Down & $\begin{array}{l}\text { Cluster of cDNA } \\
\text { FL51914, highly similar } \\
\text { to Protein DEK } \\
\text { OS=Homo sapiens PE=2 } \\
\text { SV=1 } \\
\text { (B4DNW3_HUMAN) }\end{array}$ & 0.047 & Up \\
\hline $\begin{array}{l}\text { Cluster of Tubulin beta- } \\
4 A \text { chain } O S=H o m o \\
\text { sapiens GN=TUBB4A } \\
P E=1 \mathrm{SV}=2 \\
\text { (TBB4A_HUMAN) }\end{array}$ & 0.041 & Down & $\begin{array}{l}\text { Cytochrome c oxidase } \\
\text { subunit } 4 \text { isoform } 1 \text {, } \\
\text { mitochondrial } O S=\text { Homo } \\
\text { sapiens } \mathrm{GN}=\mathrm{COX} 4 \mathrm{I} 1 \\
\mathrm{PE}=1 \mathrm{SV}=1\end{array}$ & 0.048 & Up \\
\hline $\begin{array}{l}\text { Pyridoxal kinase } \\
\text { OS=Homo sapiens } \\
\text { GN=PDXK PE=1 SV=1 }\end{array}$ & 0.041 & Down & $\begin{array}{l}\text { Nuclear transport factor } \\
2 \text { OS=Homo sapiens } \\
\text { GN=NUTF2 PE=1 SV=1 }\end{array}$ & 0.048 & Up \\
\hline $\begin{array}{l}\text { Cluster of cDNA, } \\
\text { FL96580, highly similar } \\
\text { to Homo sapiens } \\
\text { hepatoma-derived } \\
\text { growth factor (high- } \\
\text { mobility group protein 1- } \\
\text { like) (HDGF), mRNA } \\
\text { OS=Homo sapiens PE=2 } \\
\text { SV=1 (B2RDE8_HUMAN) }\end{array}$ & 0.042 & Down & $\begin{array}{l}\text { E3 ubiquitin-protein } \\
\text { ligase DTX3L OS=Homo } \\
\text { sapiens GN=DTX3L PE=1 } \\
\text { SV=1 }\end{array}$ & 0.049 & Up \\
\hline
\end{tabular}




\begin{tabular}{|l|l|l|l|l|l|l|}
\hline $\begin{array}{l}\text { Heterogeneous nuclear } \\
\text { ribonucleoprotein D0 } \\
\text { (Fragment) OS=Homo } \\
\text { sapiens GN=HNRNPD } \\
\text { PE=1 SV=8 }\end{array}$ & 0.042 & Down & & $\begin{array}{l}\text { Cluster of Reticulon-4 } \\
\text { OS=Homo sapiens } \\
\text { GN=RTN4 PE=1 SV=2 } \\
\text { (RTN4_HUMAN) }\end{array}$ & 0.05 & Up \\
\hline
\end{tabular}


Table 11 Proteome response of HT29 cells to treatment with cycloheximide from the perspective of a gene ontological (GO) analysis

\begin{tabular}{|c|c|c|c|}
\hline $\begin{array}{l}\text { Cycloheximide } \\
\text { UPs }\end{array}$ & & & \\
\hline source & term name & $\begin{array}{l}\text { adjusted } p \\
\text { value }\end{array}$ & term size \\
\hline GO:BP & $\begin{array}{l}\text { RNA splicing, via transesterification reactions } \\
\text { with bulged adenosine as nucleophile }\end{array}$ & 0.045679 & 334 \\
\hline GO:BP & mRNA splicing, via spliceosome & 0.045679 & 334 \\
\hline GO:BP & RNA splicing, via transesterification reactions & 0.048032 & 337 \\
\hline GO:CC & ribonucleoprotein complex & $\begin{array}{l}1.53 \times 10- \\
05\end{array}$ & 862 \\
\hline GO:CC & catalytic step 2 spliceosome & $\begin{array}{l}6.10 \times 10- \\
05\end{array}$ & 85 \\
\hline GO:CC & spliceosomal complex & $\begin{array}{l}8.82 \times 10- \\
05\end{array}$ & 176 \\
\hline
\end{tabular}

\begin{tabular}{|l|l|l|l|}
\hline $\begin{array}{l}\text { Cycloheximide } \\
\text { DOWNs }\end{array}$ & & & \\
\hline source & term name & $\begin{array}{l}\text { adjusted p } \\
\text { value }\end{array}$ & term size \\
\hline GO:MF & ubiquitin protein ligase binding & 0.011285 & 286 \\
\hline GO:MF & ubiquitin-like protein ligase binding & 0.015028 & 301 \\
\hline GO:MF & peptidase activator activity & 0.031093 & 39 \\
\hline GO:MF & ubiquitin-like protein conjugating enzyme activity & 0.036163 & 41 \\
\hline GO:BP & protein catabolic process & $\begin{array}{l}3.72 \times 10- \\
05\end{array}$ & 911 \\
\hline GO:BP & regulation of protein catabolic process & 0.000107 & 372 \\
\hline GO:BP & $\begin{array}{l}\text { positive regulation of protein modification by } \\
\text { small protein conjugation or removal }\end{array}$ & 0.000463 & 127 \\
\hline GO:BP & $\begin{array}{l}\text { regulation of protein modification by small } \\
\text { protein conjugation or removal }\end{array}$ & 0.000619 & 220 \\
\hline GO:BP & $\begin{array}{l}\text { proteolysis involved in cellular protein catabolic } \\
\text { process }\end{array}$ & 0.002204 & 696 \\
\hline GO:BP & cellular protein catabolic process & 0.004378 & 751 \\
\hline GO:BP & proteasomal protein catabolic process & 0.00669 & 451 \\
\hline GO:BP & $\begin{array}{l}\text { regulation of proteolysis involved in cellular } \\
\text { protein catabolic process }\end{array}$ & 0.007841 & 206 \\
\hline GO:BP & regulation of catabolic process & 0.016192 & 870 \\
\hline GO:BP & regulation of cellular protein catabolic process & 0.017517 & 237 \\
\hline GO:BP & $\begin{array}{l}\text { proteasom } x \text { 10-mediated ubiquitin-dependent } \\
\text { protein catabolic process }\end{array}$ & 0.027965 & 391 \\
\hline GO:CC & proteasome complex & 0.002105 & 67 \\
\hline GO:CC & endopeptidase complex & 0.002233 & 68 \\
\hline GO:CC & peptidase complex & 0.006792 & 90 \\
\hline & & & \\
\hline & & & \\
\hline
\end{tabular}




\begin{tabular}{|l|l|l|l|}
\hline GO:CC & ubiquitin conjugating enzyme complex & 0.03161 & 9 \\
\hline GO:CC & MCM complex & 0.048174 & 11 \\
\hline CORUM & PA28-20S proteasome & 0.046775 & 16 \\
\hline CORUM & MCM4-MCM6-MCM7 complex & 0.049917 & 3 \\
\hline CORUM & OTUB1-UBC13-MMS2 complex & 0.049917 & 3 \\
\hline
\end{tabular}

Table 12 Proteome response of HT29 cells to treatment with ribavirin from the perspective of a GO analysis

\begin{tabular}{|l|l|l|l|}
\hline Ribavirin UPs & & & \\
\hline source & term name & $\begin{array}{l}\text { adjusted } p \\
\text { value }\end{array}$ & term size \\
\hline GO:MF & cadherin binding involved in cell-cell adhesion & 0.001339 & 19 \\
\hline GO:MF & cadherin binding & 0.004298 & 323 \\
\hline GO:MF & cell-cell adhesion mediator activity & 0.026171 & 50 \\
\hline GO:MF & cell adhesion mediator activity & 0.042976 & 59 \\
\hline GO:MF & cell adhesion molecule binding & 0.043532 & 489 \\
\hline GO:CC & cell-cell adherens junction & 0.005655 & 112 \\
\hline GO:CC & adherens junction & 0.032212 & 537 \\
\hline GO:CC & proteasome complex & 0.033012 & 67 \\
\hline GO:CC & endopeptidase complex & 0.034499 & 68 \\
\hline GO:CC & anchoring junction & 0.037428 & 552 \\
\hline & & & \\
\hline & & & \\
\hline
\end{tabular}

\begin{tabular}{|l|l|l|l|}
\hline $\begin{array}{l}\text { Ribavirin } \\
\text { DOWNs }\end{array}$ & & & \\
\hline source & term name & $\begin{array}{l}\text { adjusted } \mathrm{p} \\
\text { value }\end{array}$ & term size \\
\hline GO:MF & structural constituent of ribosome & 0.004931 & 168 \\
\hline GO:MF & helicase activity & 0.039684 & 150 \\
\hline GO:BP & protein targeting to ER & $6.04 \mathrm{E}-05$ & 108 \\
\hline GO:BP & $\begin{array}{l}\text { establishment of protein localization to } \\
\text { endoplasmic reticulum }\end{array}$ & $7.78 \mathrm{E}-05$ & 112 \\
\hline GO:BP & mRNA metabolic process & $9.37 \mathrm{E}-05$ & 784 \\
\hline GO:BP & ribonucleoprotein complex biogenesis & 0.000134 & 447 \\
\hline GO:BP & $\begin{array}{l}\text { nuclear-transcribed mRNA catabolic process, } \\
\text { nonsense mediated decay }\end{array}$ & 0.000141 & 122 \\
\hline GO:BP & protein localization to endoplasmic reticulum & 0.000296 & 136 \\
\hline GO:BP & $\begin{array}{l}\text { SRP-dependent cotranslational protein targeting } \\
\text { to membrane }\end{array}$ & 0.000772 & 96 \\
\hline GO:BP & cotranslational protein targeting to membrane & 0.001043 & 101 \\
\hline GO:BP & $\begin{array}{l}\text { Nucleobase containing compound catabolic } \\
\text { process }\end{array}$ & 0.001124 & 553 \\
\hline GO:BP & RNA catabolic process & 0.001463 & 345 \\
\hline
\end{tabular}




\begin{tabular}{|l|l|l|l|}
\hline GO:BP & heterocycle catabolic process & 0.002584 & 602 \\
\hline GO:BP & cellular nitrogen compound catabolic process & 0.002626 & 603 \\
\hline GO:BP & protein targeting to membrane & 0.002847 & 190 \\
\hline GO:BP & aromatic compound catabolic process & 0.003232 & 616 \\
\hline GO:BP & RNA processing & 0.00351 & 902 \\
\hline GO:BP & rRNA processing & 0.004007 & 200 \\
\hline GO:BP & organic cyclic compound catabolic process & 0.005355 & 649 \\
\hline GO:BP & nuclear-transcribed mRNA catabolic process & 0.005538 & 210 \\
\hline GO:BP & mRNA catabolic process & 0.007079 & 311 \\
\hline GO:BP & rRNA metabolic process & 0.012281 & 237 \\
\hline GO:BP & regulation of mRNA metabolic process & 0.025407 & 265 \\
\hline GO:BP & maturation of LSU-rRNA & 0.0314 & 17 \\
\hline GO:BP & ribosome biogenesis & 0.037944 & 282 \\
\hline GO:CC & ribonucleoprotein complex & $2.81 \mathrm{E}-08$ & 862 \\
\hline GO:CC & cytosolic ribosome & 0.000273 & 119 \\
\hline GO:CC & nucleolus & 0.000371 & 934 \\
\hline GO:CC & polysome & 0.000582 & 75 \\
\hline GO:CC & cytosolic part & 0.001415 & 244 \\
\hline GO:CC & ribosomal subunit & 0.004585 & 194 \\
\hline GO:CC & ribosome & 0.017723 & 247 \\
\hline KEGG & Ribosome & 0.002587 & 149 \\
\hline CORUM & Nop56p-associated pre-rRNA complex & 0.001674 & 104 \\
\hline CORUM & Ribosome, cytoplasmic & 0.040959 & 80 \\
\hline & & & \\
\hline
\end{tabular}


Table 13 Proteome response of HT29 cells to treatment with 4E1RCat from the perspective of a GO analysis

\begin{tabular}{|c|c|c|c|}
\hline 4E1RCat UPs & & & \\
\hline source & term name & $\begin{array}{l}\text { adjusted } p \\
\text { value }\end{array}$ & term size \\
\hline GO:MF & structural constituent of ribosome & $\begin{array}{l}5.88 \times 10- \\
08\end{array}$ & 168 \\
\hline GO:MF & structural molecule activity & 0.010018 & 809 \\
\hline GO:BP & $\begin{array}{l}\text { SRP-dependent cotranslational protein targeting } \\
\text { to membrane }\end{array}$ & $\begin{array}{l}2.46 \times 10- \\
07\end{array}$ & 96 \\
\hline GO:BP & cotranslational protein targeting to membrane & $\begin{array}{l}3.53 \times 10- \\
07\end{array}$ & 101 \\
\hline GO:BP & peptide metabolic process & $\begin{array}{l}4.34 \times 10- \\
07\end{array}$ & 884 \\
\hline GO:BP & protein targeting to $E R$ & $\begin{array}{l}5.68 \times 10- \\
07\end{array}$ & 108 \\
\hline GO:BP & $\begin{array}{l}\text { establishment of protein localization to } \\
\text { endoplasmic reticulum }\end{array}$ & $\begin{array}{l}7.35 \times 10- \\
07\end{array}$ & 112 \\
\hline GO:BP & peptide biosynthetic process & $\begin{array}{l}7.36 \times 10- \\
07\end{array}$ & 727 \\
\hline GO:BP & translational initiation & $\begin{array}{l}1.13 \times 10- \\
06\end{array}$ & 198 \\
\hline GO:BP & $\begin{array}{l}\text { nuclear-transcribed mRNA catabolic process, } \\
\text { nonsens } \times 10 \text {-mediated decay }\end{array}$ & $\begin{array}{l}1.34 \times 10- \\
06\end{array}$ & 122 \\
\hline GO:BP & mRNA catabolic process & $\begin{array}{l}1.52 \times 10- \\
06\end{array}$ & 311 \\
\hline GO:BP & protein localization to endoplasmic reticulum & $\begin{array}{l}2.89 \times 10- \\
06\end{array}$ & 136 \\
\hline GO:BP & RNA catabolic process & $\begin{array}{l}3.79 \times 10- \\
06\end{array}$ & 345 \\
\hline GO:BP & amide biosynthetic process & $\begin{array}{l}4.80 \times 10- \\
06\end{array}$ & 857 \\
\hline GO:BP & translation & $\begin{array}{l}8.83 \times 10- \\
06\end{array}$ & 704 \\
\hline GO:BP & protein targeting to membrane & $\begin{array}{l}2.97 \times 10- \\
05\end{array}$ & 190 \\
\hline GO:BP & heterocycle catabolic process & $\begin{array}{l}3.03 \times 10- \\
05\end{array}$ & 602 \\
\hline GO:BP & cellular nitrogen compound catabolic process & $\begin{array}{l}3.07 \times 10- \\
05\end{array}$ & 603 \\
\hline GO:BP & $\begin{array}{l}\text { establishment of protein localization to } \\
\text { membrane }\end{array}$ & $\begin{array}{l}3.47 \times 10- \\
05\end{array}$ & 306 \\
\hline GO:BP & aromatic compound catabolic process & $\begin{array}{l}3.76 \times 10- \\
05\end{array}$ & 616 \\
\hline GO:BP & nuclear-transcribed mRNA catabolic process & $\begin{array}{l}5.91 \times 10- \\
05\end{array}$ & 210 \\
\hline
\end{tabular}




\begin{tabular}{|c|c|c|c|}
\hline GO:BP & organic cyclic compound catabolic process & $\begin{array}{l}6.17 \times 10- \\
05\end{array}$ & 649 \\
\hline GO:BP & $\begin{array}{l}\text { nucleobas } \times 10 \text {-containing compound catabolic } \\
\text { process }\end{array}$ & 0.000224 & 553 \\
\hline GO:BP & protein localization to membrane & 0.000367 & 586 \\
\hline GO:BP & protein targeting & 0.000384 & 418 \\
\hline GO:BP & establishment of protein localization to organelle & 0.002354 & 531 \\
\hline GO:BP & mRNA metabolic process & 0.0042 & 784 \\
\hline GO:BP & ribosomal large subunit biogenesis & 0.009208 & 71 \\
\hline GO:BP & protein localization to organelle & 0.010942 & 881 \\
\hline GO:CC & cytosolic large ribosomal subunit & $\begin{array}{l}1.26 \times 10- \\
09\end{array}$ & 64 \\
\hline GO:CC & large ribosomal subunit & $\begin{array}{l}2.16 \times 10- \\
09\end{array}$ & 122 \\
\hline GO:CC & ribosome & $\begin{array}{l}1.82 \times 10- \\
08\end{array}$ & 247 \\
\hline GO:CC & ribosomal subunit & $\begin{array}{l}8.98 \times 10- \\
08\end{array}$ & 194 \\
\hline GO:CC & cytosolic ribosome & $\begin{array}{l}1.07 \times 10- \\
07\end{array}$ & 119 \\
\hline GO:CC & ribonucleoprotein complex & $\begin{array}{l}4.62 \times 10- \\
07\end{array}$ & 862 \\
\hline GO:CC & cytosolic part & $\begin{array}{l}1.57 \times 10- \\
05\end{array}$ & 244 \\
\hline GO:CC & polysome & $\begin{array}{l}2.18 \times 10- \\
05\end{array}$ & 75 \\
\hline GO:CC & polysomal ribosome & $\begin{array}{l}2.72 \times 10- \\
05\end{array}$ & 30 \\
\hline KEGG & Ribosome & $\begin{array}{l}1.68 \times 10- \\
06\end{array}$ & 149 \\
\hline CORUM & $60 \mathrm{~S}$ ribosomal subunit, cytoplasmic & $\begin{array}{l}5.48 \times 10- \\
07\end{array}$ & 47 \\
\hline CORUM & Ribosome, cytoplasmic & $\begin{array}{l}2.55 \times 10- \\
05\end{array}$ & 80 \\
\hline CORUM & Nop56p-associated pr x 10-rRNA complex & 0.000161 & 104 \\
\hline
\end{tabular}

\begin{tabular}{|l|l|l|l|}
\hline $\begin{array}{l}\text { 4E1RCat } \\
\text { DOWNs }\end{array}$ & & & \\
\hline source & term name & $\begin{array}{l}\text { adjusted } \mathrm{p} \\
\text { value }\end{array}$ & term size \\
\hline GO:CC & nucleoid & 0.001483 & 43 \\
\hline GO:CC & mitochondrial nucleoid & 0.001483 & 43 \\
\hline GO:CC & nuclear replisome & 0.042695 & 25 \\
\hline
\end{tabular}


Table 14 Proteome response of HT29 cells to treatment with DMSO from the perspective of a GO analysis

\begin{tabular}{|c|c|c|c|}
\hline DMSO UPs & & & \\
\hline source & term name & $\begin{array}{l}\text { adjusted } p \\
\text { value }\end{array}$ & term size \\
\hline GO:BP & $\begin{array}{l}\text { SRP-dependent cotranslational protein targeting } \\
\text { to membrane }\end{array}$ & $\begin{array}{l}3.20 \times 10- \\
25\end{array}$ & 96 \\
\hline GO:BP & cotranslational protein targeting to membrane & $\begin{array}{l}1.24 \times 10- \\
24\end{array}$ & 101 \\
\hline GO:BP & protein targeting to ER & $\begin{array}{l}7.24 \times 10- \\
24\end{array}$ & 108 \\
\hline GO:BP & $\begin{array}{l}\text { establishment of protein localization to } \\
\text { endoplasmic reticulum }\end{array}$ & $\begin{array}{l}1.87 \times 10- \\
23\end{array}$ & 112 \\
\hline GO:BP & protein localization to endoplasmic reticulum & $\begin{array}{l}9.38 \times 10- \\
23\end{array}$ & 136 \\
\hline GO:BP & $\begin{array}{l}\text { nuclear-transcribed mRNA catabolic process, } \\
\text { nonsens } \times 10 \text {-mediated decay }\end{array}$ & $\begin{array}{l}1.71 \times 10- \\
22\end{array}$ & 122 \\
\hline GO:BP & translational initiation & $\begin{array}{l}7.82 \times 10- \\
20\end{array}$ & 198 \\
\hline GO:BP & translation & $\begin{array}{l}4.04 \times 10- \\
19\end{array}$ & 704 \\
\hline GO:BP & peptide biosynthetic process & $\begin{array}{l}1.34 \times 10- \\
18\end{array}$ & 727 \\
\hline GO:BP & protein targeting to membrane & $\begin{array}{l}1.07 \times 10- \\
17\end{array}$ & 190 \\
\hline GO:BP & cellular nitrogen compound catabolic process & $\begin{array}{l}1.14 \times 10- \\
17\end{array}$ & 603 \\
\hline GO:BP & aromatic compound catabolic process & $\begin{array}{l}2.35 \times 10- \\
17\end{array}$ & 616 \\
\hline GO:BP & $\begin{array}{l}\text { nucleobas } \times 10 \text {-containing compound catabolic } \\
\text { process }\end{array}$ & $\begin{array}{l}5.73 \times 10- \\
17\end{array}$ & 553 \\
\hline GO:BP & RNA catabolic process & $\begin{array}{l}6.68 \times 10- \\
17\end{array}$ & 345 \\
\hline GO:BP & amide biosynthetic process & $\begin{array}{l}7.67 \times 10- \\
17\end{array}$ & 857 \\
\hline GO:BP & heterocycle catabolic process & $\begin{array}{l}9.86 \times 10- \\
17\end{array}$ & 602 \\
\hline GO:BP & nuclear-transcribed mRNA catabolic process & $\begin{array}{l}1.19 \times 10- \\
16\end{array}$ & 210 \\
\hline GO:BP & peptide metabolic process & $\begin{array}{l}2.43 \times 10- \\
16\end{array}$ & 884 \\
\hline GO:BP & organic cyclic compound catabolic process & $\begin{array}{l}1.15 \times 10- \\
15\end{array}$ & 649 \\
\hline GO:BP & mRNA catabolic process & $\begin{array}{l}8.20 \times 10- \\
15\end{array}$ & 311 \\
\hline GO:BP & $\begin{array}{l}\text { establishment of protein localization to } \\
\text { membrane }\end{array}$ & $\begin{array}{l}6.63 \times 10- \\
14\end{array}$ & 306 \\
\hline
\end{tabular}




\begin{tabular}{|c|c|c|c|}
\hline GO:BP & establishment of protein localization to organelle & $\begin{array}{l}1.10 \times 10- \\
13\end{array}$ & 531 \\
\hline GO:BP & protein localization to organelle & $\begin{array}{l}2.96 \times 10- \\
12\end{array}$ & 881 \\
\hline GO:BP & protein targeting & $\begin{array}{l}1.08 \times 10- \\
11\end{array}$ & 418 \\
\hline GO:BP & protein localization to membrane & $\begin{array}{l}4.14 \times 10- \\
09\end{array}$ & 586 \\
\hline GO:BP & ncRNA metabolic process & $\begin{array}{l}4.89 \times 10- \\
09\end{array}$ & 544 \\
\hline GO:BP & mRNA metabolic process & $\begin{array}{l}2.31 \times 10- \\
08\end{array}$ & 784 \\
\hline GO:BP & rRNA metabolic process & $\begin{array}{l}6.98 \times 10- \\
06\end{array}$ & 237 \\
\hline GO:BP & DNA conformation change & $\begin{array}{l}8.04 \times 10- \\
06\end{array}$ & 278 \\
\hline GO:BP & ribosome biogenesis & $\begin{array}{l}9.86 \times 10- \\
06\end{array}$ & 282 \\
\hline GO:BP & ribonucleoprotein complex biogenesis & $\begin{array}{l}3.80 \times 10- \\
05\end{array}$ & 447 \\
\hline GO:BP & rRNA processing & $\begin{array}{l}5.93 \times 10- \\
05\end{array}$ & 200 \\
\hline GO:BP & generation of precursor metabolites and energy & $\begin{array}{l}6.66 \times 10- \\
05\end{array}$ & 463 \\
\hline GO:BP & positive regulation of gene expression, epigenetic & 0.000119 & 60 \\
\hline GO:BP & DNA metabolic process & 0.000122 & 999 \\
\hline GO:BP & nucleoside triphosphate metabolic process & 0.000125 & 294 \\
\hline GO:BP & $\begin{array}{l}\text { purine nucleoside triphosphate metabolic } \\
\text { process }\end{array}$ & 0.000363 & 275 \\
\hline GO:BP & tRNA aminoacylation for protein translation & 0.000379 & 47 \\
\hline GO:BP & ncRNA processing & 0.000401 & 368 \\
\hline GO:BP & tRNA aminoacylation & 0.000588 & 50 \\
\hline GO:BP & cellular amino acid metabolic process & 0.000629 & 333 \\
\hline GO:BP & amino acid activation & 0.000676 & 51 \\
\hline GO:BP & drug metabolic process & 0.000719 & 770 \\
\hline GO:BP & aerobic respiration & 0.000781 & 76 \\
\hline GO:BP & $\begin{array}{l}\text { nucleobas } \times 10 \text {-containing small molecule } \\
\text { metabolic process }\end{array}$ & 0.001094 & 728 \\
\hline GO:BP & nucleosome assembly & 0.001275 & 143 \\
\hline GO:BP & carboxylic acid metabolic process & 0.001329 & 989 \\
\hline GO:BP & $\begin{array}{l}\text { purine ribonucleoside triphosphate metabolic } \\
\text { process }\end{array}$ & 0.001757 & 268 \\
\hline GO:BP & ribonucleoside triphosphate metabolic process & 0.002253 & 274 \\
\hline GO:BP & nucleotide metabolic process & 0.002259 & 640 \\
\hline GO:BP & nucleoside phosphate metabolic process & 0.002547 & 645 \\
\hline GO:BP & ATP metabolic process & 0.003151 & 238 \\
\hline
\end{tabular}




\begin{tabular}{|c|c|c|c|}
\hline GO:BP & cytoplasmic translation & 0.003416 & 92 \\
\hline GO:BP & tricarboxylic acid metabolic process & 0.00381 & 42 \\
\hline GO:BP & chromatin assembly & 0.004222 & 163 \\
\hline GO:BP & chromosome condensation & 0.005052 & 44 \\
\hline GO:BP & DNA packaging & 0.005259 & 207 \\
\hline GO:BP & cellular respiration & 0.005546 & 168 \\
\hline GO:BP & interspecies interaction between organisms & 0.00649 & 811 \\
\hline GO:BP & telomere organization & 0.006503 & 171 \\
\hline GO:BP & ribosomal large subunit biogenesis & 0.006694 & 71 \\
\hline GO:BP & RNA processing & 0.010605 & 902 \\
\hline GO:BP & nucleosome organization & 0.010802 & 181 \\
\hline GO:BP & chromatin assembly or disassembly & 0.015838 & 189 \\
\hline GO:BP & nucleosome positioning & 0.015975 & 15 \\
\hline GO:BP & oxidative phosphorylation & 0.01604 & 113 \\
\hline GO:BP & $\begin{array}{l}\text { chromatin organization involved in regulation of } \\
\text { transcription }\end{array}$ & 0.01604 & 113 \\
\hline GO:BP & $\begin{array}{l}\text { doubl } \times 10 \text {-strand break repair via } \\
\text { nonhomologous end joining }\end{array}$ & 0.022388 & 85 \\
\hline GO:BP & tricarboxylic acid cycle & 0.024778 & 34 \\
\hline GO:BP & purine nucleotide metabolic process & 0.025024 & 506 \\
\hline GO:BP & telomere maintenance & 0.025356 & 158 \\
\hline GO:BP & ribose phosphate metabolic process & 0.025643 & 507 \\
\hline GO:BP & $\begin{array}{l}\text { negative regulation of gene expression, } \\
\text { epigenetic }\end{array}$ & 0.028211 & 122 \\
\hline GO:BP & non-recombinational repair & 0.037721 & 92 \\
\hline GO:BP & regulation of DNA metabolic process & 0.037729 & 409 \\
\hline GO:BP & citrate metabolic process & 0.043431 & 38 \\
\hline GO:BP & negative regulation of chromatin silencing & 0.044117 & 19 \\
\hline GO:BP & $\begin{array}{l}\text { energy derivation by oxidation of organic } \\
\text { compounds }\end{array}$ & 0.045598 & 260 \\
\hline GO:BP & purine ribonucleotide metabolic process & 0.04707 & 474 \\
\hline GO:CC & cytosolic ribosome & $\begin{array}{l}1.21 \times 10- \\
25\end{array}$ & 119 \\
\hline GO:CC & ribosome & $\begin{array}{l}5.07 \times 10- \\
25\end{array}$ & 247 \\
\hline GO:CC & ribonucleoprotein complex & $\begin{array}{l}5.01 \times 10- \\
22\end{array}$ & 862 \\
\hline GO:CC & ribosomal subunit & $\begin{array}{l}2.06 \times 10- \\
21\end{array}$ & 194 \\
\hline GO:CC & cytosolic part & $\begin{array}{l}8.23 \times 10- \\
19\end{array}$ & 244 \\
\hline GO:CC & cytosolic large ribosomal subunit & $\begin{array}{l}5.75 \times 10- \\
14\end{array}$ & 64 \\
\hline GO:CC & large ribosomal subunit & $\begin{array}{l}1.97 \times 10- \\
12\end{array}$ & 122 \\
\hline
\end{tabular}




\begin{tabular}{|c|c|c|c|}
\hline GO:CC & mitochondrial matrix & $\begin{array}{l}4.27 \times 10- \\
10\end{array}$ & 466 \\
\hline GO:CC & cytosolic small ribosomal subunit & $\begin{array}{l}2.23 \times 10- \\
09\end{array}$ & 48 \\
\hline GO:CC & nucleoid & $\begin{array}{l}2.48 \times 10- \\
08\end{array}$ & 43 \\
\hline GO:CC & mitochondrial nucleoid & $\begin{array}{l}2.48 \times 10- \\
08\end{array}$ & 43 \\
\hline GO:CC & protein-DNA complex & $\begin{array}{l}6.31 \times 10- \\
08\end{array}$ & 203 \\
\hline GO:CC & organelle inner membrane & $\begin{array}{l}1.03 \times 10- \\
07\end{array}$ & 506 \\
\hline GO:CC & small ribosomal subunit & $\begin{array}{l}2.33 \times 10- \\
07\end{array}$ & 75 \\
\hline GO:CC & focal adhesion & $\begin{array}{l}5.03 \times 10- \\
07\end{array}$ & 404 \\
\hline GO:CC & cell-substrate adherens junction & $\begin{array}{l}5.69 \times 10- \\
07\end{array}$ & 407 \\
\hline GO:CC & cell-substrate junction & $\begin{array}{l}6.68 \times 10- \\
07\end{array}$ & 411 \\
\hline GO:CC & mitochondrial envelope & $\begin{array}{l}6.75 \times 10- \\
07\end{array}$ & 723 \\
\hline GO:CC & mitochondrial membrane & $\begin{array}{l}9.85 \times 10- \\
07\end{array}$ & 680 \\
\hline GO:CC & chromosomal part & $\begin{array}{l}1.22 \times 10- \\
06\end{array}$ & 924 \\
\hline GO:CC & mitochondrial inner membrane & $\begin{array}{l}2.83 \times 10- \\
06\end{array}$ & 449 \\
\hline GO:CC & polysome & $\begin{array}{l}4.38 \times 10- \\
06\end{array}$ & 75 \\
\hline GO:CC & nucleolus & $\begin{array}{l}6.48 \times 10- \\
06\end{array}$ & 934 \\
\hline GO:CC & pigment granule & $\begin{array}{l}6.51 \times 10- \\
06\end{array}$ & 105 \\
\hline GO:CC & melanosome & $\begin{array}{l}6.51 \times 10- \\
06\end{array}$ & 105 \\
\hline GO:CC & nucleosome & $\begin{array}{l}9.35 \times 10- \\
06\end{array}$ & 109 \\
\hline GO:CC & adherens junction & $\begin{array}{l}9.47 \times 10- \\
06\end{array}$ & 537 \\
\hline GO:CC & anchoring junction & $\begin{array}{l}1.49 \times 10- \\
05\end{array}$ & 552 \\
\hline GO:CC & nuclear chromosome & $\begin{array}{l}1.51 \times 10- \\
05\end{array}$ & 608 \\
\hline GO:CC & DNA packaging complex & $\begin{array}{l}1.85 \times 10- \\
05\end{array}$ & 117 \\
\hline
\end{tabular}




\begin{tabular}{|c|c|c|c|}
\hline GO:CC & nuclear chromosome, telomeric region & $\begin{array}{l}3.21 \times 10- \\
05\end{array}$ & 124 \\
\hline GO:CC & polysomal ribosome & $\begin{array}{l}5.28 \times 10- \\
05\end{array}$ & 30 \\
\hline GO:CC & mitochondrial protein complex & 0.000108 & 260 \\
\hline GO:CC & nuclear chromosome part & 0.000119 & 570 \\
\hline GO:CC & chromosome, telomeric region & 0.00029 & 157 \\
\hline GO:CC & $\begin{array}{l}\text { aminoacyl-tRNA synthetase multienzyme } \\
\text { complex }\end{array}$ & 0.000727 & 12 \\
\hline GO:CC & chromatin & 0.001508 & 553 \\
\hline GO:CC & chromosomal region & 0.002642 & 347 \\
\hline GO:CC & organelle outer membrane & 0.02339 & 209 \\
\hline GO:CC & outer membrane & 0.025162 & 211 \\
\hline GO:CC & catalytic step 2 spliceosome & 0.026484 & 85 \\
\hline GO:CC & inner mitochondrial membrane protein complex & 0.037937 & 130 \\
\hline KEGG & Ribosome & $\begin{array}{l}3.51 \times 10- \\
16\end{array}$ & 149 \\
\hline KEGG & Biosynthesis of amino acids & 0.000168 & 73 \\
\hline KEGG & Citrate cycle (TCA cycle) & 0.000521 & 30 \\
\hline KEGG & Carbon metabolism & 0.001097 & 115 \\
\hline KEGG & Parkinson disease & 0.003001 & 129 \\
\hline KEGG & Non-homologous end-joining & 0.003257 & 13 \\
\hline KEGG & Aminoacyl-tRNA biosynthesis & 0.005164 & 44 \\
\hline KEGG & Huntington disease & 0.013718 & 185 \\
\hline CORUM & Ribosome, cytoplasmic & $\begin{array}{l}1.70 \times 10- \\
16\end{array}$ & 80 \\
\hline CORUM & Nop56p-associated pr x 10-rRNA complex & $\begin{array}{l}7.48 \times 10- \\
15\end{array}$ & 104 \\
\hline CORUM & 60S ribosomal subunit, cytoplasmic & $\begin{array}{l}1.38 \times 10- \\
09\end{array}$ & 47 \\
\hline CORUM & 40S ribosomal subunit, cytoplasmic & $\begin{array}{l}1.49 \times 10- \\
05\end{array}$ & 31 \\
\hline CORUM & 40S ribosomal subunit, cytoplasmic & $\begin{array}{l}2.74 \times 10- \\
05\end{array}$ & 33 \\
\hline CORUM & $\begin{array}{l}\text { TRBP containing complex (DICER, RPL7A, EIF6, } \\
\text { MOV10 and subunits of the } 60 \text { S ribosomal } \\
\text { particle) }\end{array}$ & $\begin{array}{l}3.71 \times 10- \\
05\end{array}$ & 25 \\
\hline CORUM & Multisynthetase complex & 0.037748 & 11 \\
\hline CORUM & prohibitin 2 complex, mitochondrial & 0.049881 & 5 \\
\hline
\end{tabular}

\begin{tabular}{|l|l|l|l|}
\hline DMSO UPs & & & \\
\hline source & term name & $\begin{array}{l}\text { adjusted } \mathrm{p} \\
\text { value }\end{array}$ & term size \\
\hline GO:BP & mRNA metabolic process & $\begin{array}{l}6.35 \times 10- \\
10\end{array}$ & 784 \\
\hline
\end{tabular}




\begin{tabular}{|c|c|c|c|}
\hline GO:BP & peptide metabolic process & $\begin{array}{l}2.09 \times 10- \\
08\end{array}$ & 884 \\
\hline GO:BP & peptide biosynthetic process & $\begin{array}{l}4.72 \times 10- \\
08\end{array}$ & 727 \\
\hline GO:BP & RNA splicing & $\begin{array}{l}1.11 \times 10- \\
07\end{array}$ & 422 \\
\hline GO:BP & amide biosynthetic process & $\begin{array}{l}1.72 \times 10- \\
07\end{array}$ & 857 \\
\hline GO:BP & RNA splicing, via transesterification reactions & $\begin{array}{l}3.61 \times 10- \\
07\end{array}$ & 337 \\
\hline GO:BP & protein folding & $\begin{array}{l}4.19 \times 10- \\
07\end{array}$ & 211 \\
\hline GO:BP & translation & $\begin{array}{l}4.68 \times 10- \\
07\end{array}$ & 704 \\
\hline GO:BP & negative regulation of mRNA metabolic process & $\begin{array}{l}5.71 \times 10- \\
07\end{array}$ & 70 \\
\hline GO:BP & $\begin{array}{l}\text { nucleobas x 10-containing compound catabolic } \\
\text { process }\end{array}$ & $\begin{array}{l}1.06 \times 10- \\
06\end{array}$ & 553 \\
\hline GO:BP & regulation of mRNA metabolic process & $\begin{array}{l}1.95 \times 10- \\
06\end{array}$ & 265 \\
\hline GO:BP & mRNA splicing, via spliceosome & $\begin{array}{l}2.01 \times 10- \\
06\end{array}$ & 334 \\
\hline GO:BP & $\begin{array}{l}\text { RNA splicing, via transesterification reactions } \\
\text { with bulged adenosine as nucleophile }\end{array}$ & $\begin{array}{l}2.01 \times 10- \\
06\end{array}$ & 334 \\
\hline GO:BP & regulated exocytosis & $\begin{array}{l}2.10 \times 10- \\
06\end{array}$ & 750 \\
\hline GO:BP & aromatic compound catabolic process & $\begin{array}{l}2.22 \times 10- \\
06\end{array}$ & 616 \\
\hline GO:BP & RNA catabolic process & $\begin{array}{l}3.52 \times 10- \\
06\end{array}$ & 345 \\
\hline GO:BP & mRNA catabolic process & $\begin{array}{l}3.86 \times 10- \\
06\end{array}$ & 311 \\
\hline GO:BP & heterocycle catabolic process & $\begin{array}{l}6.31 \times 10- \\
06\end{array}$ & 602 \\
\hline GO:BP & cellular nitrogen compound catabolic process & $\begin{array}{l}6.54 \times 10- \\
06\end{array}$ & 603 \\
\hline GO:BP & organic cyclic compound catabolic process & $\begin{array}{l}6.82 \times 10- \\
06\end{array}$ & 649 \\
\hline GO:BP & neutrophil degranulation & $\begin{array}{l}8.91 \times 10- \\
06\end{array}$ & 483 \\
\hline GO:BP & $\begin{array}{l}\text { neutrophil activation involved in immune } \\
\text { response }\end{array}$ & $\begin{array}{l}1.00 \times 10- \\
05\end{array}$ & 486 \\
\hline GO:BP & symbiont process & $\begin{array}{l}1.26 \times 10- \\
05\end{array}$ & 762 \\
\hline GO:BP & exocytosis & $\begin{array}{l}1.43 \times 10- \\
05\end{array}$ & 864 \\
\hline
\end{tabular}




\begin{tabular}{|c|c|c|c|}
\hline GO:BP & neutrophil activation & $\begin{array}{l}1.47 \times 10- \\
05\end{array}$ & 496 \\
\hline GO:BP & neutrophil mediated immunity & $\begin{array}{l}1.53 \times 10- \\
05\end{array}$ & 497 \\
\hline GO:BP & granulocyte activation & $\begin{array}{l}1.91 \times 10- \\
05\end{array}$ & 503 \\
\hline GO:BP & viral process & $\begin{array}{l}2.99 \times 10- \\
05\end{array}$ & 696 \\
\hline GO:BP & leukocyte degranulation & $\begin{array}{l}4.86 \times 10- \\
05\end{array}$ & 529 \\
\hline GO:BP & interspecies interaction between organisms & $\begin{array}{l}4.99 \times 10- \\
05\end{array}$ & 811 \\
\hline GO:BP & $\begin{array}{l}\text { myeloid cell activation involved in immune } \\
\text { response }\end{array}$ & $\begin{array}{l}6.63 \times 10- \\
05\end{array}$ & 538 \\
\hline GO:BP & protein localization to organelle & $\begin{array}{l}8.29 \times 10- \\
05\end{array}$ & 881 \\
\hline GO:BP & nuclear transport & $\begin{array}{l}8.35 \times 10- \\
05\end{array}$ & 337 \\
\hline GO:BP & mRNA processing & $\begin{array}{l}8.40 \times 10- \\
05\end{array}$ & 501 \\
\hline GO:BP & interleukin-12-mediated signaling pathway & $\begin{array}{l}8.47 \times 10- \\
05\end{array}$ & 47 \\
\hline GO:BP & myeloid leukocyte mediated immunity & $\begin{array}{l}8.68 \times 10- \\
05\end{array}$ & 546 \\
\hline GO:BP & $\begin{array}{l}\text { negative regulation of mRNA splicing, via } \\
\text { spliceosome }\end{array}$ & 0.000115 & 20 \\
\hline GO:BP & cellular response to interleukin-12 & 0.000119 & 49 \\
\hline GO:BP & response to interleukin-12 & 0.000141 & 50 \\
\hline GO:BP & regulation of mRNA stability & 0.000173 & 119 \\
\hline GO:BP & regulation of catabolic process & 0.000226 & 870 \\
\hline GO:BP & establishment of protein localization to organelle & 0.000232 & 531 \\
\hline GO:BP & regulation of RNA stability & 0.000339 & 127 \\
\hline GO:BP & nucleocytoplasmic transport & 0.000401 & 334 \\
\hline GO:BP & RNA processing & 0.000482 & 902 \\
\hline GO:BP & negative regulation of RNA splicing & 0.000501 & 25 \\
\hline GO:BP & drug metabolic process & 0.000856 & 770 \\
\hline GO:BP & regulation of mRNA catabolic process & 0.000978 & 141 \\
\hline GO:BP & myeloid leukocyte activation & 0.001267 & 635 \\
\hline GO:BP & negative regulation of mRNA processing & 0.001295 & 29 \\
\hline GO:BP & protein localization to nucleus & 0.001379 & 247 \\
\hline GO:BP & $\begin{array}{l}\text { leukocyte activation involved in immune } \\
\text { response }\end{array}$ & 0.001623 & 694 \\
\hline GO:BP & cytoplasmic translation & 0.001634 & 92 \\
\hline GO:BP & negative regulation of programmed cell death & 0.001733 & 960 \\
\hline GO:BP & cell activation involved in immune response & 0.001797 & 698 \\
\hline
\end{tabular}




\begin{tabular}{|c|c|c|c|}
\hline GO:BP & cellular amino acid metabolic process & 0.001986 & 333 \\
\hline GO:BP & regulation of cellular catabolic process & 0.002288 & 759 \\
\hline GO:BP & $\begin{array}{l}\text { positive regulation of protein localization to Cajal } \\
\text { body }\end{array}$ & 0.003443 & 9 \\
\hline GO:BP & regulation of protein localization to Cajal body & 0.003443 & 9 \\
\hline GO:BP & RNA localization & 0.003762 & 231 \\
\hline GO:BP & negative regulation of apoptotic process & 0.004064 & 946 \\
\hline GO:BP & translational initiation & 0.004373 & 198 \\
\hline GO:BP & ribonucleoprotein complex assembly & 0.00455 & 235 \\
\hline GO:BP & regulation of RNA splicing & 0.004889 & 134 \\
\hline GO:BP & protein stabilization & 0.004922 & 166 \\
\hline GO:BP & ATP metabolic process & 0.005235 & 238 \\
\hline GO:BP & chaperon $\times 10$-mediated protein folding & 0.005321 & 56 \\
\hline GO:BP & protein localization to Cajal body & 0.005688 & 10 \\
\hline GO:BP & $\begin{array}{l}\text { positive regulation of establishment of protein } \\
\text { localization to telomere }\end{array}$ & 0.005688 & 10 \\
\hline GO:BP & protein localization to nuclear body & 0.005688 & 10 \\
\hline GO:BP & positive regulation of organelle organization & 0.007231 & 602 \\
\hline GO:BP & cofactor metabolic process & 0.007491 & 554 \\
\hline GO:BP & ribonucleoprotein complex subunit organization & 0.008596 & 249 \\
\hline GO:BP & $\begin{array}{l}\text { regulation of establishment of protein } \\
\text { localization to telomere }\end{array}$ & 0.008861 & 11 \\
\hline GO:BP & carboxylic acid metabolic process & 0.0095 & 989 \\
\hline GO:BP & nucleoside triphosphate metabolic process & 0.010643 & 294 \\
\hline GO:BP & protein export from nucleus & 0.01261 & 183 \\
\hline GO:BP & $\begin{array}{l}\text { regulation of establishment of protein } \\
\text { localization to chromosome }\end{array}$ & 0.013176 & 12 \\
\hline GO:BP & $\begin{array}{l}\text { positive regulation of protein localization to } \\
\text { chromosome, telomeric region }\end{array}$ & 0.013176 & 12 \\
\hline GO:BP & protein localization to nucleoplasm & 0.013176 & 12 \\
\hline GO:BP & $\begin{array}{l}\text { proteasomal ubiquitin-independent protein } \\
\text { catabolic process }\end{array}$ & 0.013791 & 25 \\
\hline GO:BP & ribonucleotide metabolic process & 0.01796 & 489 \\
\hline GO:BP & $\begin{array}{l}\text { purine ribonucleoside triphosphate metabolic } \\
\text { process }\end{array}$ & 0.019033 & 268 \\
\hline GO:BP & regulation of translation & 0.019468 & 398 \\
\hline GO:BP & $\begin{array}{l}\text { purin } \times 10 \text {-containing compound metabolic } \\
\text { process }\end{array}$ & 0.019549 & 541 \\
\hline GO:BP & ribonucleoside triphosphate metabolic process & 0.024107 & 274 \\
\hline GO:BP & $\begin{array}{l}\text { purine nucleoside triphosphate metabolic } \\
\text { process }\end{array}$ & 0.02506 & 275 \\
\hline GO:BP & sulfur compound metabolic process & 0.02604 & 362 \\
\hline GO:BP & $\begin{array}{l}\text { regulation of protein localization to chromosome, } \\
\text { telomeric region }\end{array}$ & 0.026184 & 14 \\
\hline
\end{tabular}




\begin{tabular}{|c|c|c|c|}
\hline GO:BP & nuclear export & 0.026583 & 198 \\
\hline GO:BP & positive regulation of catabolic process & 0.027289 & 409 \\
\hline GO:BP & regulation of mRNA splicing, via spliceosome & 0.028151 & 99 \\
\hline GO:BP & purine nucleotide metabolic process & 0.028473 & 506 \\
\hline GO:BP & negative regulation of protein polymerization & 0.029089 & 72 \\
\hline GO:BP & ribose phosphate metabolic process & 0.029236 & 507 \\
\hline GO:BP & supramolecular fiber organization & 0.029339 & 660 \\
\hline GO:BP & generation of precursor metabolites and energy & 0.033165 & 463 \\
\hline GO:BP & cellular aldehyde metabolic process & 0.034877 & 74 \\
\hline GO:BP & $\begin{array}{l}\text { positive regulation of telomerase RNA localization } \\
\text { to Cajal body }\end{array}$ & 0.035395 & 15 \\
\hline GO:BP & cytoplasmic translational initiation & 0.041777 & 31 \\
\hline GO:BP & purine ribonucleotide metabolic process & 0.044737 & 474 \\
\hline GO:CC & cytoplasmic vesicle lumen & $\begin{array}{l}5.76 \times 10- \\
12\end{array}$ & 336 \\
\hline GO:CC & vesicle lumen & $\begin{array}{l}6.17 \times 10- \\
12\end{array}$ & 337 \\
\hline GO:CC & secretory granule lumen & $\begin{array}{l}1.58 \times 10- \\
11\end{array}$ & 319 \\
\hline GO:CC & adherens junction & $\begin{array}{l}1.49 \times 10- \\
10\end{array}$ & 537 \\
\hline GO:CC & anchoring junction & $\begin{array}{l}2.96 \times 10- \\
10\end{array}$ & 552 \\
\hline GO:CC & ribonucleoprotein complex & $\begin{array}{l}7.94 \times 10- \\
09\end{array}$ & 862 \\
\hline GO:CC & cell-substrate junction & $\begin{array}{l}2.42 \times 10- \\
08\end{array}$ & 411 \\
\hline GO:CC & focal adhesion & $\begin{array}{l}1.09 \times 10- \\
07\end{array}$ & 404 \\
\hline GO:CC & cell-substrate adherens junction & $\begin{array}{l}1.26 \times 10- \\
07\end{array}$ & 407 \\
\hline GO:CC & ficolin-1-rich granule lumen & $\begin{array}{l}2.10 \times 10- \\
07\end{array}$ & 123 \\
\hline GO:CC & ficolin-1-rich granule & $\begin{array}{l}2.10 \times 10- \\
07\end{array}$ & 123 \\
\hline GO:CC & cytosolic part & $\begin{array}{l}2.45 \times 10- \\
06\end{array}$ & 244 \\
\hline GO:CC & chaperone complex & $\begin{array}{l}7.91 \times 10- \\
06\end{array}$ & 18 \\
\hline GO:CC & chaperonin-containing T-complex & $\begin{array}{l}1.12 \times 10- \\
05\end{array}$ & 10 \\
\hline GO:CC & proteasome complex & $\begin{array}{l}1.23 \times 10- \\
05\end{array}$ & 67 \\
\hline GO:CC & endopeptidase complex & $\begin{array}{l}1.41 \times 10- \\
05\end{array}$ & 68 \\
\hline
\end{tabular}




\begin{tabular}{|c|c|c|c|}
\hline GO:CC & contractile fiber part & $\begin{array}{l}2.59 \times 10- \\
05\end{array}$ & 216 \\
\hline GO:CC & contractile fiber & $\begin{array}{l}6.28 \times 10- \\
05\end{array}$ & 232 \\
\hline GO:CC & supramolecular fiber & $\begin{array}{l}6.60 \times 10- \\
05\end{array}$ & 941 \\
\hline GO:CC & actin cytoskeleton & $\begin{array}{l}6.82 \times 10- \\
05\end{array}$ & 482 \\
\hline GO:CC & supramolecular polymer & $\begin{array}{l}7.71 \times 10- \\
05\end{array}$ & 948 \\
\hline GO:CC & supramolecular complex & $\begin{array}{l}7.88 \times 10- \\
05\end{array}$ & 949 \\
\hline GO:CC & secretory vesicle & 0.000144 & 977 \\
\hline GO:CC & zona pellucida receptor complex & 0.000155 & 7 \\
\hline GO:CC & peptidase complex & 0.000165 & 90 \\
\hline GO:CC & myofibril & 0.000224 & 220 \\
\hline GO:CC & secretory granule & 0.000232 & 831 \\
\hline GO:CC & cell-cell junction & 0.000491 & 450 \\
\hline GO:CC & spliceosomal complex & 0.000983 & 176 \\
\hline GO:CC & cytoplasmic stress granule & 0.001033 & 59 \\
\hline GO:CC & proteasome core complex & 0.001678 & 24 \\
\hline GO:CC & sarcomere & 0.003199 & 199 \\
\hline GO:CC & cell cortex part & 0.003342 & 163 \\
\hline GO:CC & cytoplasmic region & 0.004385 & 476 \\
\hline GO:CC & cell cortex & 0.004522 & 289 \\
\hline GO:CC & cortical cytoskeleton & 0.004924 & 103 \\
\hline GO:CC & perinuclear region of cytoplasm & 0.004951 & 693 \\
\hline GO:CC & hemidesmosome & 0.014924 & 7 \\
\hline GO:CC & myelin sheath & 0.025036 & 166 \\
\hline GO:CC & polymeric cytoskeletal fiber & 0.026699 & 719 \\
\hline GO:CC & ribonucleoprotein granule & 0.040086 & 218 \\
\hline KEGG & Spliceosome & 0.00028 & 132 \\
\hline KEGG & Proteasome & 0.000299 & 45 \\
\hline CORUM & $26 \mathrm{~S}$ proteasome & 0.000344 & 22 \\
\hline CORUM & $20 \mathrm{~S}$ proteasome & 0.006425 & 14 \\
\hline CORUM & $\begin{array}{l}\text { CCT complex (chaperonin containing TCP1 } \\
\text { complex) }\end{array}$ & 0.009222 & 8 \\
\hline CORUM & PA28gamma-20S proteasome & 0.009421 & 15 \\
\hline CORUM & PA700-20S-PA28 complex & 0.012117 & 36 \\
\hline CORUM & PA28-20S proteasome & 0.013395 & 16 \\
\hline CORUM & PABPC1-HSPA8-HNRPD-EIF4G1 complex & 0.020393 & 4 \\
\hline CORUM & BBS-chaperonin complex & 0.026472 & 10 \\
\hline CORUM & Multiprotein complex (mRNA turnover) & 0.049927 & 5 \\
\hline
\end{tabular}




\section{References}

Argilés, J. M., Busquets, S., Stemmler, B., \& López-Soriano, F. J. (2014). Cancer cachexia: Understanding the molecular basis. Nature Reviews Cancer, 14(11), 754-762. https://doi.org/10.1038/nrc3829

Asara, J. M., Christofk, H. R., Freimark, L. M., \& Cantley, L. C. (2008). A label-free quantification method by MS/MS TIC compared to SILAC and spectral counting in a proteomics screen. Proteomics, 8(5), 994-999. https://doi.org/10.1002/pmic.200700426

Barabási, A.-L., Gulbahce, N., \& Loscalzo, J. (2011). An Integrative Systems Medicine Approach to Mapping Human Metabolic Diseases. Nat Rev Genet, 12(1), 56-68. https://doi.org/10.1038/nrg2918.Network

Birnie, G. D. (1988). The HL60 cell line: A model system for studying human myeloid cell differentiation. British Journal of Cancer.

Bordeleau, M.-E., Matthews, J., Wojnar, J. M., Lindqvist, L., Novac, O., Jankowsky, E., ... Pelletier, J. (2005). Stimulation of mammalian translation initiation factor elF4A activity by a small molecule inhibitor of eukaryotic translation. Proceedings of the National Academy of Sciences, 102(30), 10460-10465. https://doi.org/10.1073/pnas.0504249102

Cencic, R., Hall, D. R., Robert, F., Du, Y., Min, J., Li, L., ... Lewis, I. (2010). Reversing chemoresistance by small molecule inhibition of the translation initiation complex elF4F. Proceedings of the National Academy of Sciences, 108(3), 1046-1051. https://doi.org/10.1073/pnas.1011477108

Cencic, R., \& Pelletier, J. (2016). Hippuristanol - A potent steroid inhibitor of eukaryotic initiation factor 4A. Trans/ation, 4(1), e1137381. https://doi.org/10.1080/21690731.2015.1137381

Chambers, J. M., Lindqvist, L. M., Webb, A., Huang, D. C. S., Savage, G. P., \& Rizzacasa, M. A. (2013). Synthesis of Biotinylated Episilvestrol : Highly Selective Targeting of the Translation Factors elF4AI / II, (7), 13-16. https://doi.org/10.1021/ol400401d

Cho, P. F., Osler, W., \& Hg, Q. (2008). Cap-Dependent Translational Inhibition Establishes Two Opposing Morphogen Gradients in Drosophila Embryos, 16(20), 2035-2041.

Clark, A. L., Coats, A. J. S., Krum, H., Katus, H. A., Mohacsi, P., Salekin, D., ... Anker, S. D. (2017). Effect of beta-adrenergic blockade with carvedilol on cachexia in severe chronic heart failure : results from the COPERNICUS trial, (February), 549-556. https://doi.org/10.1002/jcsm.12191

Cramer, Z., Sadek, J., Vazquez, G. G., Di Marco, S., Pause, A., Pelletier, J., \& Gallouzi, I. E. (2018). EIF4A inhibition prevents the onset of cytokin $\times 10$-induced muscle wasting by blocking the STAT3 and iNOS pathways. Scientific Reports, 8(1), 2-11. https://doi.org/10.1038/s41598-01826625-9

Crotty, S., Cameron, C. E., \& Andino, R. (2001). RNA virus error catastrophe : Direct molecular test by using ribavirin, 98(12).

Di Marco, S., Cammas, A., Lian, X. J., Kovacs, E. N., Ma, J. F., Hall, D. T., ... Gallouzi, I. E. (2012). The translation inhibitor pateamine A prevents cachexia-induced muscle wasting in mice. Nature Communications, 3(May), 812-896. https://doi.org/10.1038/ncomms1899

Dobs, A. S. (2013). Effects of enobosarm on muscle wasting and physical function in patients with cancer: a doubl x 10-blind, randomised controlled phase 2 trial. Lancet Oncology, 4(14), 335345. https://doi.org/10.1183/09031936.00063810.The 
Duffy, A. G., Makarova-Rusher, O. V., \& Ulahannan, S. V. (2016). Modulation of tumor elF4E by antisense inhibition: A phase I/II translational clinical trial of ISIS 183750-an antisense oligonucleotide against elF4E -in combination with irinotecan in solid tumors and irinotecanrefractory colorectal cancer, 1657, 1648-1657. https://doi.org/10.1002/ijc.30199

Evans, W. J., Morley, J. E., Argilés, J., Bales, C., Baracos, V., Guttridge, D., ... Anker, S. D. (2008). Cachexia: A new definition. Clinical Nutrition, 27(6), 793-799.

https://doi.org/10.1016/j.clnu.2008.06.013

Frey, N. P., \& Davis, E. P. (2016). Launch and commissioning the deep space climate observatory. Advances in the Astronautical Sciences, 157(October 2013), 999-1010. https://doi.org/10.1002/pbc

Frydryskova, K., Masek, T., Borcin, K., Mrvova, S., Venturi, V., \& Pospisek, M. (2016). Distinct recruitment of human elF4E isoforms to processing bodies and stress granules. BMC Molecular Biology, 17(1), 1-19. https://doi.org/10.1186/s12867-016-0072-x

Gordon, J. N., Trebble, T. M., Ellis, R. D., Duncan, H. D., Johns, T., \& Goggin, P. M. (2005). Thalidomide in the treatment of cancer cachexia: a randomised placebo controlled trial, 540-545. https://doi.org/10.1136/gut.2004.047563

Harms, U., Andreou, A. Z., Gubaev, A., \& Klostermeier, D. (2014). EIF4B, elF4G and RNA regulate elF4A activity in translation initiation by modulating the elF4A conformational cycle. Nucleic Acids Research, 42(12), 7911-7922. https://doi.org/10.1093/nar/gku440

Hayano, T., Yanagida, M., Yamauchi, Y., Shinkawa, T., Isobe, T., \& Takahashi, N. (2003). Proteomic Analysis of Human Nop56p-associated $\operatorname{Pr} \times 10$-ribosomal Ribonucleoprotein Complexes. Journal of Biological Chemistry, 278(36), 34309-34319. https://doi.org/10.1074/jbc.m304304200

Hemi Cumming, A., Brown, S. L., Tao, X., Cuyamendous, C., Field, J. J., Miller, J. H., ... Teesdal x 10Spittle, P. H. (2016). Synthesis of a simplified triazole analogue of pateamine A. Organic and Biomolecular Chemistry, 14(22), 5117-5127. https://doi.org/10.1039/c6ob00086j

Ho, J., Hyun, Y., Young, S., Min, J., Kim, A., \& Chang, Y. (2009). Crystal structure of the elF4A - PDCD4 complex, 1-6.

Iwasaki, S., Floor, S. N., \& Ingolia, N. T. (2016). Rocaglates convert DEAD-box protein elF4A into a sequenc x 10-selective translational repressor. Nature, 534(7608), 558-561. https://doi.org/10.1038/nature17978

Iwasaki, S., Iwasaki, W., Takahashi, M., Sakamoto, A., Watanabe, C., Shichino, Y., ... Ingolia, N. T. (2019). The Translation Inhibitor Rocaglamide Targets a Bimolecular Cavity between elF4A and Polypurine RNA. Molecular Cell, 73(4), 738-748.e9.

https://doi.org/10.1016/j.molcel.2018.11.026

Joshi, B., Lee, K., Maeder, D. L., \& Jagus, R. (2005). Phylogenetic analysis of elF4 X 10-family members, 20, 1-20. https://doi.org/10.1186/1471-2148-5-48

Katakami, N., Uchino, J., Yokoyama, T., Naito, T., Kondo, M., Yamada, K., ... Eguchi, K. (2018). Anamorelin (ONO-7643) for the treatment of patients with non-small cell lung cancer and cachexia: Results from a randomized, doubl x 10-blind, placebo-controlled, multicenter study of Japanese patients (ONO-7643-04). Cancer, 124(3), 606-616.

https://doi.org/10.1002/cncr.31128

Kentsis, A., Topisirovic, I., Culjkovic, B., Shao, L., \& Borden, K. L. B. (2004). Ribavirin suppresses elF4 X 10-mediated oncogenic transformation by physical mimicry of the 7-methyl guanosine mRNA cap, 101(52), 18105-18110. 
Kentsis, A., Volpon, L., Topisirovic, I., Soll, C. E., Culjkovic, B., Shao, L., \& Borden, K. L. B. (2005). Further evidence that ribavirin interacts with elF4E. Rna, 11(12), 1762-1766.

https://doi.org/10.1261/rna.2238705

Kislinger, T., Gramolini, A. O., MacLennan, D. H., \& Emili, A. (2005). Multidimensional protein identification technology (MudPIT): Technical overview of a profiling method optimized for the comprehensive proteomic investigation of normal and diseased heart tissue. Journal of the American Society for Mass Spectrometry, 16(8), 1207-1220.

https://doi.org/10.1016/j.jasms.2005.02.015

Korneeva, N. L., Lamphear, B. J., Hennigan, F. L. C., Merrick, W. C., \& Rhoads, R. E. (2001). Characterization of the Two elF4A-binding Sites on Human elF4G-1. Journal of Biological Chemistry, 276(4), 2872-2879. https://doi.org/10.1074/jbc.M006345200

Kuznetsov, G., Xu, Q., Rudolph-Owen, L., TenDyke, K., Liu, J., Towle, M., ... Littlefield, B. A. (2009). Potent in vitro and in vivo anticancer activities of des-methyl, des-amino pateamine $A, a$ synthetic analogue of marine natural product pateamine A. Molecular Cancer Therapeutics, 8(5), 1250-1260. https://doi.org/10.1158/1535-7163.MCT-08-1026

Lanford, R. E., Hong, Z., Chavez, D., Guerra, B., Lau, J. Y. N., Beames, B., \& Brasky, K. M. (2002). Ribavirin Induces Error-Prone Replication of GB Virus B in Primary Tamarin Hepatocytes. Journal of Virology, 75(17), 8074-8081. https://doi.org/10.1128/jvi.75.17.8074-8081.2001

Le Hir, H., Gatfield, D., Izaurralde, E., \& Moore, M. J. (2001). The exon-exon junction complex provides a binding platform for factors involved in mRNA export and nonsens $\times 10$-mediated mRNA decay. EMBO Journal, 20(17), 4987-4997. https://doi.org/10.1093/emboj/20.17.4987

Leyssen, P., Clercq, E. De, \& Neyts, J. (2006). The Anti-Yellow Fever Virus Activity of Ribavirin Is Independent of Error-Prone Replication, 69(4), 1461-1467.

https://doi.org/10.1124/mol.105.020057.for

Li, S., \& Rousseau, D. (2012). ATAD3, a vital membrane bound mitochondrial ATPase involved in tumor progression. Journal of Bioenergetics and Biomembranes, 44(1), 189-197. https://doi.org/10.1007/s10863-012-9424-5

Lindqvist, L., Oberer, M., Reibarkh, M., Cencic, R., Bordeleau, M. E., Vogt, E., ... Pelletier, J. (2008). Selective pharmacological targeting of a DEAD box RNA helicase. PLOS ONE, 3(2). https://doi.org/10.1371/journal.pone.0001583

Litonin, D., Sologub, M., Shi, Y., Savkina, M., Anikin, M., Falkenberg, M., ... Temiakov, D. (2010). Human Mitochondrial Transcription Revisited. Journal of Biological Chemistry, 285(24), 1812918133. https://doi.org/10.1074/jbc.C110.128918

Low, W. K., Dang, Y., Bhat, S., Romo, D., \& Liu, J. O. (2007). Substrat x 10-Dependent Targeting of Eukaryotic Translation Initiation Factor $4 \mathrm{~A}$ by Pateamine A: Negation of Domain-Linker Regulation of Activity. Chemistry and Biology, 14(6), 715-727. https://doi.org/10.1016/j.chembiol.2007.05.012

Low, W. K., Dang, Y., Schneider-Poetsch, T., Shi, Z., Choi, N. S., Merrick, W. C., ... Liu, J. O. (2005). Inhibition of eukaryotic translation initiation by the marine natural product pateamine $A$. Molecular Cell, 20(5), 709-722. https://doi.org/10.1016/j.molcel.2005.10.008

Marcotrigiano, J., Gingras, A. C., Sonenberg, N., \& Burley, S. K. (1999). Cap-dependent translation initiation in eukaryotes is regulated by a molecular mimic of elF4G. Molecular Cell, 3(6), 707716. https://doi.org/10.1016/S1097-2765(01)80003-4

Matthews, J. H. (2010). THESIS: The Molecular Pharmacology of Pateamine A. 
Matthews, J. H., Maass, D. R., Northcote, P. T., Atkinson, P. H., \& Teesdal x 10-Spittle, P. H. (2013). The cellular target specificity of pateamine A. Zeitschrift Fur Naturforschung - Section C Journal of Biosciences, 68 C(9-10), 406-415. https://doi.org/10.1515/znc-2013-9-1008

Merrick, W. C. (1992). Mechanism and regulation of eukaryotic protein synthesis. Microbiological Reviews, 56(2), 291-315. https://doi.org/10.1101/gad.11.21.2755

Migliaccio, A., Rotondi, A., \& Auricchio, F. (2006). Calmodulin-stimulated phosphorylation of 17 betaestradiol receptor on tyrosine. Proceedings of the National Academy of Sciences, 81(19), 59215925. https://doi.org/10.1073/pnas.81.19.5921

Milne, A. C., Potter, J., \& Avenell, A. (2005). Protein and energy supplementation in elderly people at risk from malnutrition. Cochrane Database of Systematic Reviews, (2). https://doi.org/10.1002/14651858.CD003288.pub2

Minich, W. B., Balastat, M. L., Gosst, D. J., \& Rhoads, R. E. (1994). Chromatographic resolution of in vivo phosphorylated and nonphosphorylated eukaryotic translation initiation factor elF-4E : Increased cap affinity of the phosphorylated form, 91(August), 7668-7672.

Moerke, N. J., Aktas, H., Chen, H., Cantel, S., Reibarkh, M. Y., Fahmy, A., ... Wagner, G. (2007). SmallMolecule Inhibition of the Interaction between the Translation Initiation Factors elF4E and elF4G. Cell, 128(2), 257-267. https://doi.org/10.1016/j.cell.2006.11.046

Morin, P. J., Vogelstein, B., \& Kinzlertt, K. W. (1996). Apoptosis and APC in colorectal tumorigenesis. Medical Sciences, 93(July), 7950-7954. https://doi.org/10.1073/pnas.93.15.7950

Morino, S., Imataka, H., Svitkin, Y. V, Pestova, T. V, \& Sonenberg, N. (2000). Eukaryotic translation initiation factor $4 \mathrm{E}$ (elF4E) binding site and the middle on $\times 10$-third of elF4GI constitute the core domain for cap-dependent translation, and the C-terminal on $\times 10$-third functions as a modulatory region. Molecular \& Cellular Biology, 20(2), 468-477. https://doi.org/10.1128/MCB.20.2.468-477.2000

Morley, J., Thomas, D., \& Wilson, M.-M. (2006). Cachexia: Pathophysiology and clinical relevance. American Journal of Clinical Nutrition, 83(4), 735-743. https://doi.org/10.1093/ajcn/83.4.735

Naiki, M., Ochi, N., Kato, Y. S., Purevsuren, J., Yamada, K., Kimura, R., ... Wakamatsu, N. (2014). Mutations in $\mathrm{HADHB}$, which encodes the $\beta$-subunit of mitochondrial trifunctional protein, cause infantile onset hypoparathyroidism and peripheral polyneuropathy. American Journal of Medical Genetics, Part A, 164(5), 1180-1187. https://doi.org/10.1002/ajmg.a.36434

Ngagore, L., Nadeau, R. J., Guo, Q., Jadhav, Y., Jarrett, H., \& Haskins, W. (2013). Purification \& Characterization of Transcription Factors, 5(32), 386-398.

https://doi.org/10.1002/bmb.20244.DNA

Nielsen, P. J., \& Trachsel, H. (1988). The mouse protein synthesis initiation factor 4A gene family includes two related functional genes which are differentially expressed. The EMBO Journal, 7(7), 2097-2105. https://doi.org/10.1093/jxb/erx229

Northcote, P. T., Blunt, J. W., \& Munro, M. H. G. (1991). Pateamine: a potent cytotoxin from the New Zealand Marine sponge, mycale sp. Tetrahedron Letters, 32(44), 6411-6414. https://doi.org/10.1016/0040-4039(91)80182-6

Oberer, M., Marintchev, A., \& Wagner, G. (2005). Structural basis for the enhancement of elF4A helicase activity by elF4G. Genes and Development, 19(18), 2212-2223.

https://doi.org/10.1101/gad.1335305

Oguro, A., Ohtsu, T., Svitkin, Y. V, Sonenberg, N., \& Nakamura, Y. (2003). RNA aptamers to initiation 
factor 4A helicase hinder cap-dependent translation by blocking ATP hydrolysis, 394-407. https://doi.org/10.1261/rna.2161303.)

Owen, J. G., Charlop-Powers, Z., Smith, A. G., Ternei, M. A., Calle, P. Y., Reddy, B. V. B., ... Brady, S. F. (2015). Multiplexed metagenome mining using short DNA sequence tags facilitates targeted discovery of epoxyketone proteasome inhibitors. Proceedings of the National Academy of Sciences, 112(14), 4221-4226. https://doi.org/10.1073/pnas.1501124112

Page, M. J., Handley, S. J., Northcote, P. T., Cairney, D., \& Willan, R. C. (2011). Successes and pitfalls of the aquaculture of the sponge Mycale hentscheli. Aquaculture, 312(1-4), 52-61. https://doi.org/10.1016/j.aquaculture.2010.12.006

Page, T., \& Connor, J. D. (1990). The metabolism of ribavirin in erythrocytes and nucleated cells. International Journal of Biochemistry, 22(4), 379-383. https://doi.org/10.1016/0020711X(90)90140-X

Pelletier, J., Graff, J., Ruggero, D., \& Sonenberg, N. (2015). TARGETING THE elF4F TRANSLATION INITIATION COMPLEX: A CRITICAL NEXUS FOR CANCER DEVELOPMENT. Cancer Research, 75(2), 250-263. https://doi.org/10.1158/0008-5472.CAN-14-2789

Pestova, T. V, \& Kolupaeva, V. G. (2002). The roles of individual eukaryotic translation initiation factors in ribosomal scanning and initiation codon selection, 3, 2906-2922. https://doi.org/10.1101/gad.1020902.5

Peters, T. L., Tillotson, J., Yeomans, A. M., Wilmore, S., Lemm, E., Jimenez-Romero, C., ... Schatz, J. H. (2018). Target-based screening against elF4A1 reveals the marine natural product elatol as a novel inhibitor of translation initiation with in vivo antitumor activity. Clinical Cancer Research, 24(17), 4256-4270. https://doi.org/10.1158/1078-0432.CCR-17-3645

Pichon, X., A. Wilson, L., Stoneley, M., Bastide, A., A King, H., Somers, J., \& E Willis, A. (2012). RNA Binding Protein/RNA Element Interactions and the Control of Translation. Current Protein \& Peptide Science, 13(4), 294-304. https://doi.org/10.2174/138920312801619475

Rakesh, R., Joseph, A. P., Bhaskara, R. M., \& Srinivasan, N. (2016). Structural and mechanistic insights into human splicing factor SF3b complex derived using an integrated approach guided by the cryo-EM density maps. RNA Biology, 13(10), 1025-1040.

https://doi.org/10.1080/15476286.2016.1218590

Reid, M. B., \& Li, Y. P. (2001). Tumor necrosis factor- $\alpha$ and muscle wasting: A cellular perspective. Respiratory Research, 2(5), 269-272. https://doi.org/10.1186/rr67

Reimand, J., Arak, T., Adler, P., Kolberg, L., Reisberg, S., Peterson, H., \& Vilo, J. (2016). g:Profiler-a web server for functional interpretation of gene lists (2016 update). Nucleic Acids Research, 44(W1), W83-W89. https://doi.org/10.1093/nar/gkw199

Rendón-Huerta, E. P., Torres-Martínez, A., Charles-Niño, C., Rivas-Estilla, A. M., Paez, A., Fortoul, T. I., \& Montaño, L. F. (2013). Pegylated interferon- $\alpha 2 b$ and ribavirin decrease claudin-1 and X 10cadherin expression in HepG2 and Huh-7.5 cells. Annals of Hepatology, 12(4), 448-457.

Romo, D., Choi, N. S., Li, S., Buchler, I., Shi, Z., \& Liu, J. O. (2004). Evidence for separate binding and scaffolding domains in the immunosuppressive and antitumor marine natural product, pateamine A: Design, synthesis, and activity studies leading to a potent simplified derivative. Journal of the American Chemical Society, 126(34), 10582-10588. https://doi.org/10.1021/ja040065s

Romo, D., Rzasa, R. M., Shea, H. A., Park, K., Langenhan, J. M., Sun, L., ... Liu, J. O. (1998). Total synthesis and immunosuppressive activity of (-)-pateamine $A$ and related compounds: 
Implementation of a $\beta$-lactam-based macrocyclization. Journal of the American Chemical Society, 120(47), 12237-12254. https://doi.org/10.1021/ja981846u

Rozen, F., Edery, I., Meerovitch, K., Dever, T. E., Merrick, W. C., \& Sonenberg, N. (1990). Bidirectional RNA helicase activity of eucaryotic translation initiation factors $4 \mathrm{~A}$ and $4 \mathrm{~F}$. Molecular and Cellular Biology, 10(3), 1134-1144. https://doi.org/10.1128/MCB.10.3.1134

Ryu, A. H., Eckalbar, W. L., Kreimer, A., Yosef, N., \& Ahituv, N. (2017). Use antibiotics in cell culture with caution: Genom $\times 10$-wide identification of antibiotic-induced changes in gene expression and regulation. Scientific Reports, 7(1), 1-9. https://doi.org/10.1038/s41598-017-07757-w

Sadlish, H., Galicia-vazquez, G., Paris, C. G., Aust, T., Bhullar, B., Chang, L., ... Movva, N. R. (2014). Evidence for a functionally relevant rocaglamide binding site on the elF4A:RNA complex, 8(7), 1519-1527. https://doi.org/10.1021/cb400158t.Evidence

Schneider-Poetsch, T., Ju, J., Eyler, D. E., Dang, Y., Bhat, S., Merrick, W. C., ... Liu, J. O. (2010). Inhibition of eukaryotic translation elongation by cycloheximide and lactimidomycin. Nature Chemical Biology, 6(3), 209-217. https://doi.org/10.1038/nchembio.304

Schwanhäusser, B., Wolf, J., Chen, W., Selbach, M., Busse, D., Dittmar, G., ... Schuchhardt, J. (2011). Global quantification of mammalian gene expression control. Nature, 473(7347), 337-342. https://doi.org/10.1038/nature10098

Serkan Kir, S., White, J. P., Kleiner, S., Kazak, L., Cohen, P., Baracos, aVikie E., \& Spiegelman, B. M. (2014). Tumor-derived PTHrP Triggers Adipose Tissue Browning and Cancer Cachexia. Nature, 513(7516), 100-104. https://doi.org/10.1038/nature13528.Tumor-derived

Speicher, D. W. (2010). Target-Decoy Search Strategy for Mass Spectrometry-Based Proteomics. Current Protocols in Protein Science, (5), 25.0.1-25.0.2. https://doi.org/10.1002/0471140864.ps2500s60

Sun, Y., Atas, E., Lindqvist, L. M., Sonenberg, N., Pelletier, J., \& Meller, A. (2014). Singl x 10-Molecule Kinetics of the Eukaryotic Initiation Factor 4AI upon RNA Unwinding. Structure/Folding and Design, 22(7), 941-948. https://doi.org/10.1016/j.str.2014.04.014

Svitkin, Y., Pause, A., Haghighat, A., \& Pyronnet, S. (2001). The requirement for eukaryotic initiation factor $4 \mathrm{~A}$ (elF4A) in translation is in direct proportion to .... Rna, (2001), 382-394. Retrieved from http://www.ncbi.nlm.nih.gov/entrez/query.fcgi?db=pubmed\&cmd=Retrieve\&dopt=AbstractPlu s\&list_uids=10684923970642830528related:wOQ4er15SJQJ\%5Cnhttp://journals.cambridge.or g/abstract_S135583820100108X

Szklarczyk, D., Gable, A. L., Lyon, D., Junge, A., Wyder, S., Huerta-Cepas, J., ... Von Mering, C. (2019). STRING v11: Protein-protein association networks with increased coverage, supporting functional discovery in genom x 10-wide experimental datasets. Nucleic Acids Research, 47(D1), D607-D613. https://doi.org/10.1093/nar/gky1131

Szklarczyk, D., Morris, J. H., Cook, H., Kuhn, M., Wyder, S., Simonovic, M., ... Von Mering, C. (2017). The STRING database in 2017: Quality-controlled protein-protein association networks, made broadly accessible. Nucleic Acids Research, 45(D1), D362-D368.

https://doi.org/10.1093/nar/gkw937

Tanabe, M., \& Kanehisa, M. (2012). Using the KEGG database resource. Current Protocols in Bioinformatics. https://doi.org/10.1002/0471250953.bi0112s38

Tao, X. (2015). Tristetraprolin Recruits Eukaryotic Initiation Factor 4E2 To Repress Translation of AURich Element-Containing mRNAs, 35(22), 3921-3932. https://doi.org/10.1128/MCB.00845- 


\section{Address}

Timm, M., Saaby, L., Moesby, L., \& Hansen, E. W. (2013). Considerations regarding use of solvents in in vitro cell based assays. Cytotechnology, 65(5), 887-894. https://doi.org/10.1007/s10616012-9530-6

Trudgian, D. C., Fischer, R., Guo, X., Kessler, B. M., \& Mirzaei, H. (2014). GOAT - A simple LC-MS/MS gradient optimization tool. Proteomics, 14(12), 1467-1471.

https://doi.org/10.1002/pmic.201300524

Vanhoutte, G., Van De Wiel, M., Wouters, K., Sels, M., Bartolomeeussen, L., De Keersmaecker, S., ... Peeters, M. (2016). Cachexia in cancer: what is in the definition? BMJ Open Gastroenterology, 3(1), 1-11. https://doi.org/10.1136/bmjgast-2016-000097

Venturi, V., Little, R., Bircham, P. W., Rodigheri Brito, J., Atkinson, P. H., Maass, D. R., \& Teesdal x 10Spittle, P. H. (2018). Characterisation of the biological response of Saccharomyces cerevisiae to the loss of an allele of the eukaryotic initiation factor 4A. Biochemical and Biophysical Research Communications, 496(4), 1082-1087. https://doi.org/10.1016/j.bbrc.2018.01.137

Verheijen, M. C. T., Lienhard, M., Schrooders, Y. J. M., Clayton, O., Nudischer, R., Timmermann, B., ... Caiment, F. (2018). DMSO-induced drastic changes in cellular processes and epigenetic landscape in vitro. Toxicology Letters, 295(August 2018), S215. https://doi.org/10.1016/j.toxlet.2018.06.927

Volpin, F., Casaos, J., Sesen, J., Mangraviti, A., Choi, J., Gorelick, N., ... Skuli, N. (2017). Use of an antiviral drug, Ribavirin, as an anti-glioblastoma therapeutic. Oncogene, 36(21), 3037-3047. https://doi.org/10.1038/onc.2016.457

Von Haehling, S., \& Anker, S. D. (2015). Treatment of cachexia: An overview of recent developments. International Journal of Cardiology, 184(1), 726-742. https://doi.org/10.1016/j.ijcard.2014.10.026

Waldron, J. A., Raza, F., \& Le Quesne, J. (2018). elF4A alleviates the translational repression mediated by classical secondary structures more than by G-quadruplexes. Nucleic Acids Research, 46(6), 3075-3087. https://doi.org/10.1093/nar/gky108

Warren S. (1932). 1932 Warren - The Immediate Causes of Death in Cancer.pdf. Am J Med Sci.

Westman, B., Beeren, L., Grudzien, E., Stepinski, J., Worch, R., Zuberek, J., ... Preiss, T. (2005). The antiviral drug ribavirin does not mimic the 7-methylguanosine moiety of the mRNA cap structure in vitro. Rna, 11(10), 1505-1513. https://doi.org/10.1261/rna.2132505

Williams-Hill, D. M., Duncan, R. F., Nielsen, P. J., \& Tahara, S. M. (1997). Differential expression of the murine eukaryotic translation initiation factor isogenes elF4A(I) and elF4A(II) is dependent upon cellular growth status. Archives of Biochemistry and Biophysics, 338(1), 111-120. https://doi.org/10.1006/abbi.1996.9804

Wolfe, A. L., Singh, K., Zhong, Y., Drewe, P., Rajasekhar, V. K., Sanghvi, V. R., ... Rätsch, G. (2015). RNA G-quadruplexes cause elF4A-dependent oncogene translation in cancer, 513(7516), 65-70. https://doi.org/10.1038/nature13485.RNA

Yan, Y., Svitkin, Y., Lee, J. M., Bisaillon, M., \& Pelletier, J. (2005). Ribavirin is not a functional mimic of the 7-methyl guanosine mRNA cap. Rna, 11(8), 1238-1244. https://doi.org/10.1261/rna.2930805

Yeeles, J. T. P., Janska, A., Early, A., \& Diffley, J. F. X. (2017). How the Eukaryotic Replisome Achieves Rapid and Efficient DNA Replication. Molecular Cell, 65(1), 105-116. 
https://doi.org/10.1016/j.molcel.2016.11.017

Yoder-Hill, J., Pause, A., Sonenberg, N., \& Merrick, W. C. (1993). The p46 subunit of eukaryotic initiation factor (elF)-4F exchanges with elF-4A. Journal of Biological Chemistry, 268(8), 55665573. https://doi.org/10.1006/BBRC.1995.1958

Yu, J. G., Bonnerud, P., Eriksson, A., Stal, P. S., Tegner, Y., \& Malm, C. (2014). Effects of long term supplementation of anabolic androgen steroids on human skeletal muscle. PLOS ONE, 9(9). https://doi.org/10.1371/journal.pone.0105330

Zhao, P., Liu, Q., Miller, W. A., \& Goss, D. J. (2017). Eukaryotic translation initiation factor 4G (elF4G) coordinates interactions with elF4A, elF4B, and elF4E in binding and translation of the barley yellow dwarf virus $3^{\prime}$ cap-independent translation element (BTE). Journal of Biological Chemistry, 292(14), 5921-5931. https://doi.org/10.1074/jbc.M116.764902 